\title{
SENSING VEGETATION CANOPIES IN THE THERMAL DOMAIN
}


Graduation committee:

\section{Chairman/Secretary}

Prof.dr.ir. A. Veldkamp University of Twente

\section{Supervisor}

Prof.dr. A.K. Skidmore University of Twente

\section{Co-supervisors}

Dr. R. Darvishzadeh Varchehi

University of Twente Dr.ir. T.A. Groen

\section{Members}

Prof.dr. F.D. van der Meer

University of Twente

Prof.dr. Z. Su

Prof.dr. M. Herold

Prof.dr.ir. P.M. van Bodegom

University of Twente

University of Twente

Wageningen University

Leiden University

ITC dissertation number 302

ITC, P.O. Box 217, 7500 AA Enschede, The Netherlands

ISBN 978-90-365-4351-4

DOI $10.3990 / 1.9789036543514$

Cover designed by Benno Masselink

Printed by ITC Printing Department

Copyright (c) 2017 by Elnaz Neinavaz

TU FACULTY OF GEO-INFORMATION SCIENCE AND EARTH OBSERVATION 


\title{
SENSING VEGETATION CANOPIES IN THE THERMAL DOMAIN
}

\author{
DISSERTATION
}

to obtain

the degree of doctor at the University of Twente, on the authority of the rector magnificus, prof.dr. T.T.M. Palstra,

on account of the decision of the graduation committee, to be publicly defended

on Wednesday $31^{\text {st }}$ of May 2017 at $16: 45$ hrs

by

Elnaz Neinavaz

born on $12^{\text {th }}$ May 1982

in Tehran, Iran 
This thesis is approved by

Prof.dr. A. K. Skidmore, supervisor

Dr. R. Darvishzadeh Varchehi, co-supervisor

Dr.ir. T. A. Groen, co-supervisor 
Dedicated to my Family 



\section{Acknowledgements}

This work would not have been in the present form, without the tremendous support, dedication, and contribution of several individuals. I express my sincere appreciation to all of those who have contributed to this dissertation.

First and foremost, I would like to extend my deepest gratitude to my promotor Prof. Dr. Andrew K. Skidmore for motivating, guiding and supporting me through my Ph.D. I have learned a great deal about research and working manners due to his guidance. Andrew, I still remember the early days, when my measurements in the Lab were not progressing well, but your optimism about my work encouraged and reassured me. You have always supported and helped me, to stay on track. It has been an incredible honor and privilege to do my Ph.D. under your supervision.

My heartfelt thanks, go to my daily supervisor and co- promoter Dr. Roshanak Darvishzadeh who has always been available for discussion and guidance. During my research, whenever I hit a brick wall, she helped me to find a way in order to unravel my way out of it. Her office would be the first place I would visit in case of any discovery, doubt or question. Her friendly advice, critical comments, and reviews were extremely helpful, enhancing and improving the quality of my research. Roshanak, the lessons which I have learned from you, will light the path in my future career. I am thankful to Dr. Thomas A. Groen my other co-promoter for his support and help throughout my Ph.D. I appreciate his guidance and contribution during my research. His detailed and valuable comments have improved my manuscripts.

I am deeply indebted to the European Commission's Erasmus Mundus Program for awarding me a Ph.D. Scholarship and Faculty of Geoinformation Science and Earth Observation (ITC) for the financial support.

I have been fortunate to come across wonderful officemates, "room 4-110 crew" Parinaz Rashidi, Xi Zhu, Wanderi Festus, Maria Fernanda Buitrago, Yifang Shi, Amjad Ali, Phil Wilkes, John Wasige and Falk Grossmann. I would like to thank them for their friendship and valuable support. I would also like to thank Haidi Abdullah for his unreserved help and collaboration in field data collection. My sincere gratitude goes to the NRS Ph.D. candidates: Mitra Shariati Najafabadi, Sam Khosravifard, Babak Naimi, Aidin Niamir, Anahita Khosravipour, Nina Amiri, Linlin Li, Bryan Hally, Fangyuan Yu, 
Jing Liu, Trini Del Rio, Sonia Asilo, Alby Duarte Rocha, Zhihui Wang, Yifei Xue, Sugandh Chauhan and Gara Tawanda for their friendship. It has been a pleasure working with you and discussing different topics during coffee breaks or lunch. I would like to thank the NRS Department Staff, especially Esther Hondebrink for her enormous assistance and support, the Geo-science laboratory team, the ITC student affairs, the ITC library, the Travel Unit, the Facility Management Service unit and the Finance Department staff who helped me during my stay at ITC. My special thanks go to Boudewijn de Smeth, Watse Siderius and Christopher Hecker for their unconstrained and speedy support. My heartfelt gratitude goes to Loes Colenbrander for her incredible support in arranging all kind of logistical matters, whenever I knocked at the door of her office. I appreciate all the help and support from Theresa van den Boogaard and Lyande Eelderink. I would like to mention and thank, Roelof Schoppers at the front desk at ITC reception who always greets everyone with a good morning and brings a smile to their face. I am very thankful to Eva Skidmore and Joy Burrough-Boenisch whose excellent English editing helped me to publish papers.

My sincerest thanks go to my generation of Ph.D. candidates. It was great to be part of such a splendid, and multicultural group. Due to the long tenure of $\mathrm{Ph} . \mathrm{D}$. candidateship, it is hard to mention all the friends and colleagues. However, among them Xiaoling Wang, Matthew Dimal, Milad Mahour, Adish Khezri, Dimitris Stratoulias, Efthymia Pavlidou, Siddhi and Riddhi Munde, Hakan Tanyas, Thea Turkington, Sanaz Salati, Anandita Sengupta, Manuel Garcia Alvarez, Eduardo Pérez Molina and Riswan Sianturi.

Without friends in Enschede, life would have been spiritless and dull. I have been fortunate to have made wonderful friends during this period. My cordial thanks to Hamed Mehdipoor, Soodabeh Amininezhad, and Tonny and Dorien Boeve for their hospitality, the incredible food and for making my stay "a home away from home." I could not complete this dissertation without the support of my amazing friends no matter where they have been in this globe. My heartfelt thanks go to Leila Sadrieh for her relentless support. Leila, I am deeply grateful for your care, all joyful moments and laughs that we shared via Skype chats. You have been like a true sister to me. I would like to extend my deepest appreciation to my dearest cousins Kamand Ghadakchian and Maryam Delvarani, and lifelong friends Parinaz Sallati, and Besat Emami for their encouragement and valuable friendship. 
Last but not least, I would like to express my deepest gratitude and love to the most valuable people in my life, who have always been there for me. My wonderful parents Rouhi and Monir who have always supported me, especially at difficult times, providing me with whatever I needed and whenever I needed it. Rouhi and Monir you are the greatest "Baba" and "Maman" that I could ever wish for. You have given me tremendous support and love. I owe you everything and I will eternally be grateful to you. To my brother Yashar and my sister in law Nastaran, you have given me endless love. Yashar, your "Do you need something, baby?" questions, have always made me feel supported and at ease. This work is dedicated to my family. 


\section{Table of Contents}

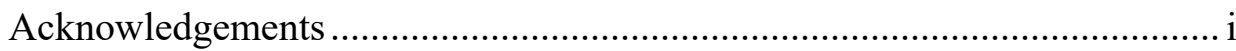

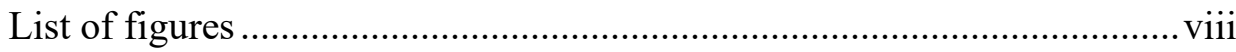

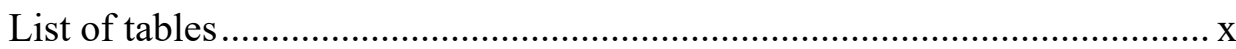

List of Abbreviation .................................................................................

Chapter 1 General Introduction................................................................. 1

1.1 Remote sensing of biophysical and biochemical characteristics .......2

1.2 Thermal remote sensing of vegetation.............................................. 4

1.3 Research objectives .....................................................................

1.4 The study area......................................................................

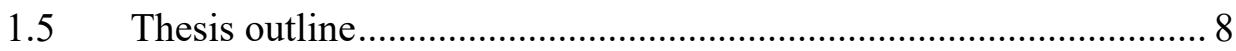

Chapter 2 Measuring the Response of Canopy Emissivity Spectra to Leaf Area Index Variation using Thermal Hyperspectral Data............................. 11

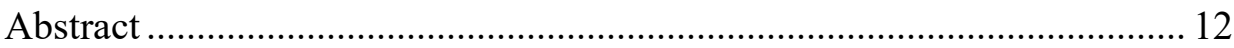

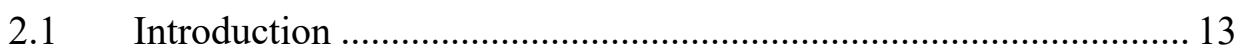

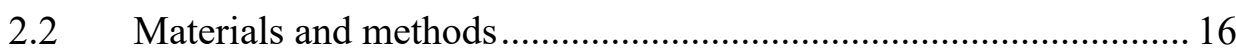

2.2.1 LAI measurements........................................................................ 16

2.2.2 Laboratory condition ………………………………………....... 16

2.2.3 Canopy spectroscopic measurement............................................... 17

2.2.3.1 Thermal infrared emission spectroscopy .......................................... 17

2.2.3.1.1 Radiometric calibration and down-welling radiance

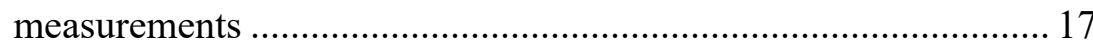

2.2.3.1.2 Canopy radiance measurements ................................................... 18

2.2.4 Data processing and analysis ........................................................ 19

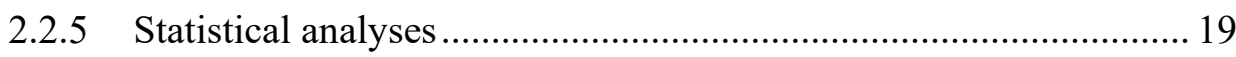

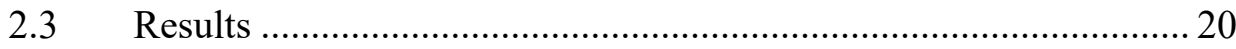

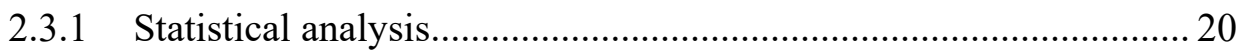

2.3.2 Principal component analysis ......................................................... 25

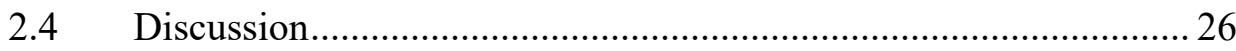

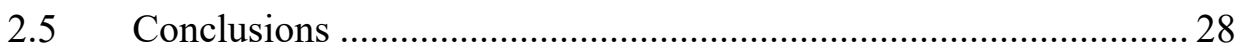

Chapter 3 Retrieval of Leaf Area Index in Different Plant Species Using Thermal Hyperspectral Data .....................................................................29

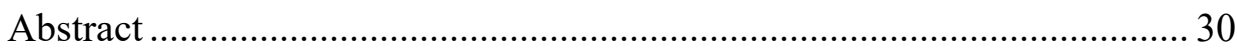

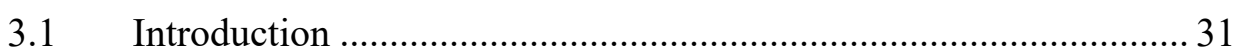

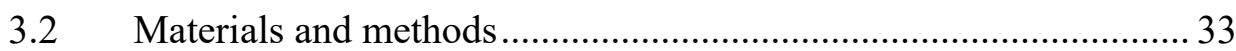

3.2.1 Leaf area index measurements........................................................... 33 
3.2.2 Canopy thermal infrared radiance measurement …............................34

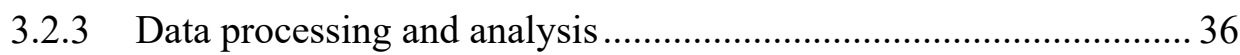

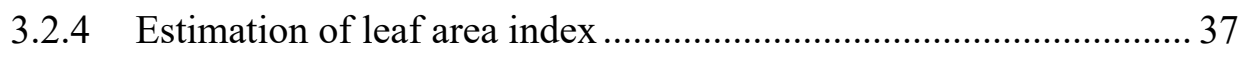

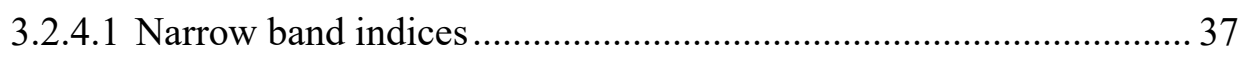

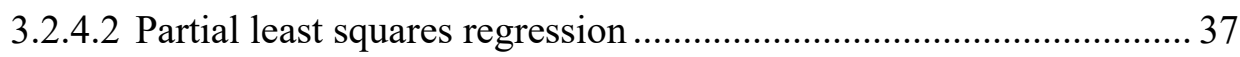

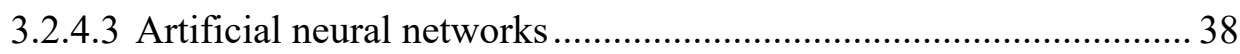

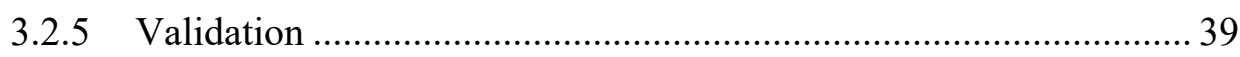

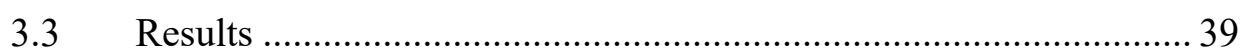

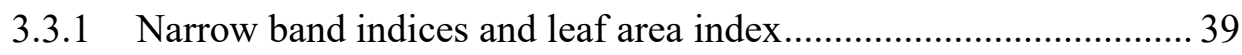

3.3.2 Partial least squares regression and leaf area index........................ 46

3.3.3 Artificial neural networks and leaf area index................................ 49

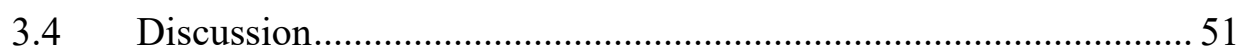

3.5 Conclusions ................................................................................ 54

Chapter 4 Retrieving Vegetation Canopy Water Content from

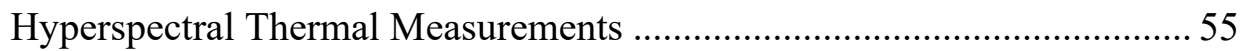

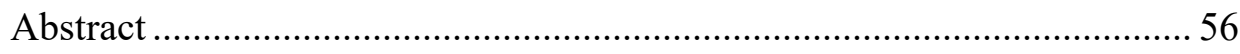

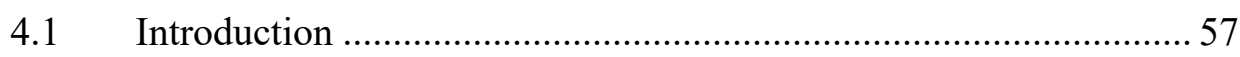

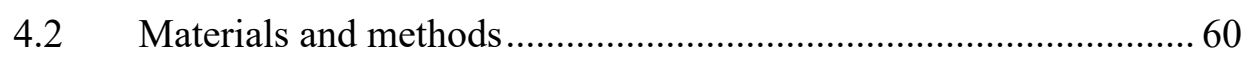

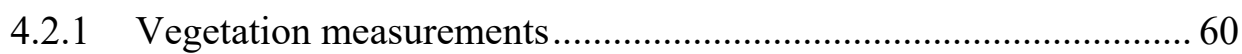

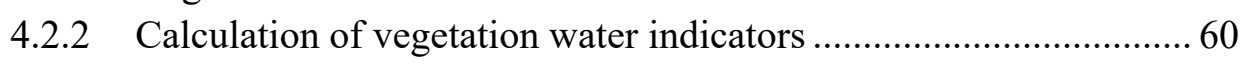

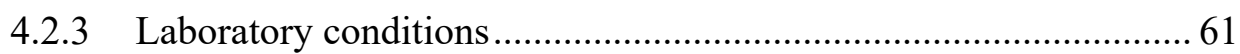

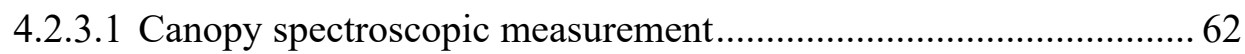

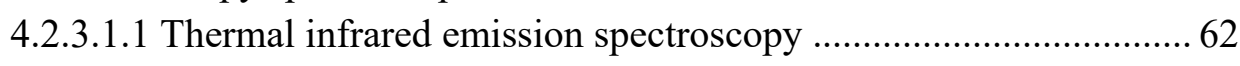

4.2.3.1.2 Radiometric calibration and down-welling radiance

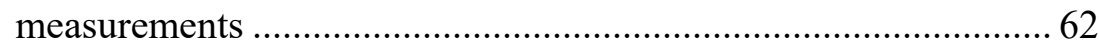

4.2.3.2 Canopy radiance measurements .....................................................6 63

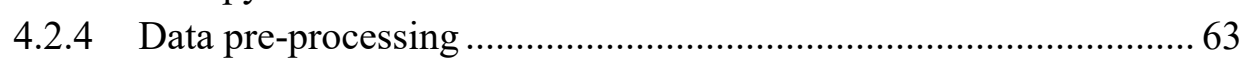

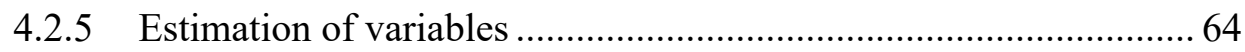

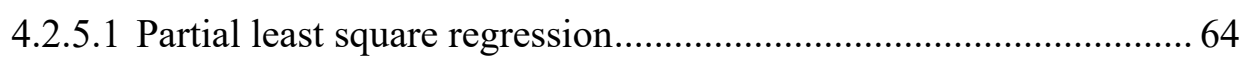

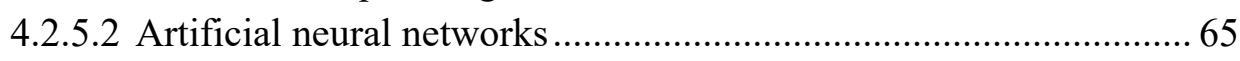

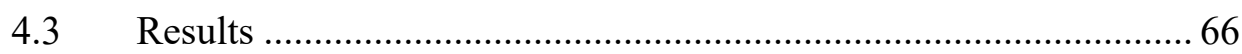

4.3.1 Relationship between canopy variables and emissivity spectra ..... 66

4.3.2 Partial least square regression and vegetation water content

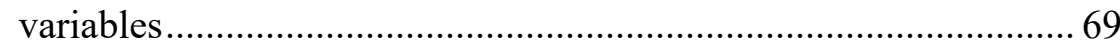

4.3.3 Estimating vegetation water content using artificial neural

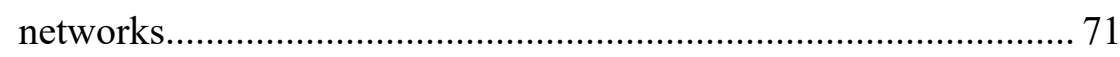




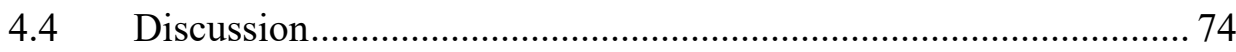

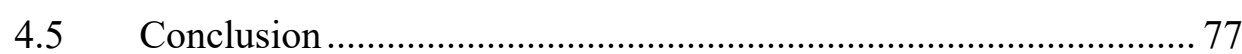

Chapter 5 Successful Retrieval of Leaf Area Index from Thermal Satellite Imagery over Mixed Temperate Forest.................................................. 79

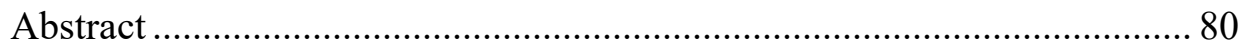

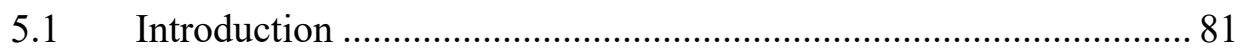

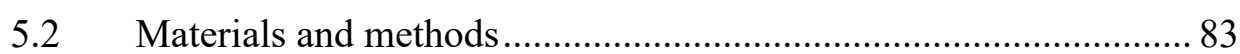

5.2.1 General description of the study area ........................................... 83

5.2.2 In situ, structural canopy parameter collection ................................. 84

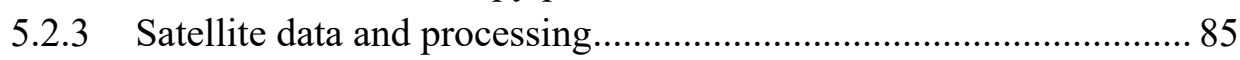

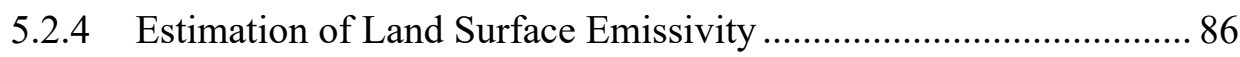

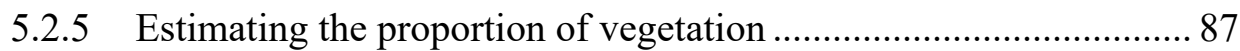

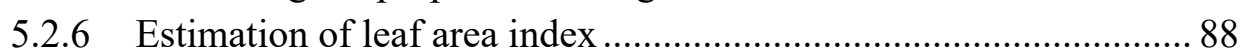

5.2.6.1 Estimation of leaf area index using vegetation indices .................... 88

5.2.6.2 Estimation of leaf area index using artificial neural networks ........ 89

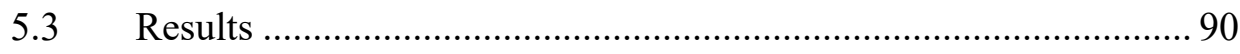

5.3.1 Leaf area index and proportion of vegetation................................. 90

5.3.2 Estimation of proportion of vegetation cover................................... 90

5.3.3 Retrieval of land surface emissivity ............................................. 92

5.3.4 Relationships between leaf area index and land surface emissivity 95

5.3.5 Estimation of leaf area index using vegetation indices in the thermal

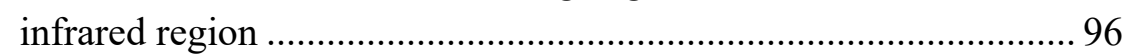

5.3.6 Estimating leaf area index using artificial neural networks............. 97

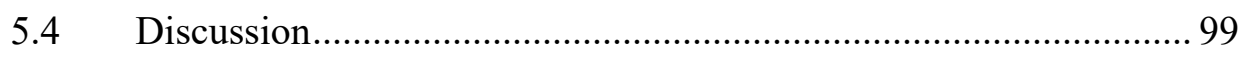

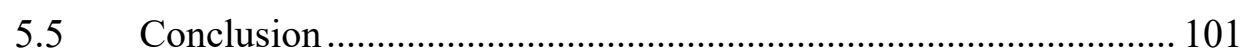

Chapter 6 Synthesis: Sensing Vegetation Canopies in the Thermal

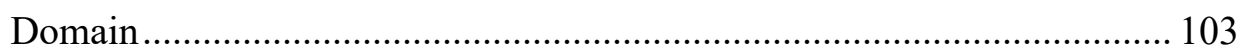

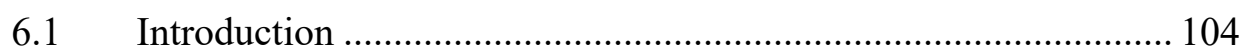

6.2 Measuring the response of canopy emissivity spectra to leaf area index variation using thermal hyperspectral data ......................... 105

6.3 Retrieval of leaf area index in different plant species using thermal hyperspectral data .................................................................... 106

6.4 Retrieving vegetation canopy water content from hyperspectral

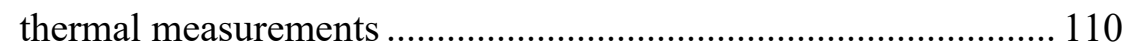

6.5 Successful retrieval of leaf area index from thermal satellite imagery over mixed temperate forest 
6.6 Border implications of using thermal infrared remotely sensed data for estimation of vegetation parameters in ecological studies....... 116

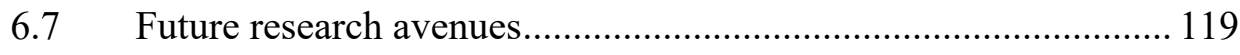

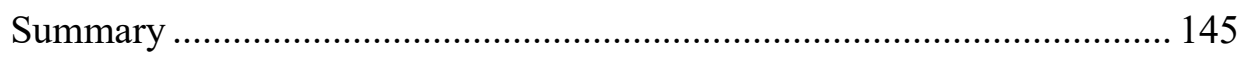

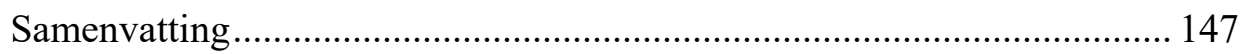

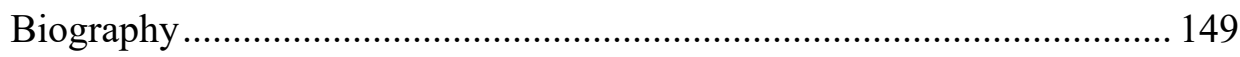

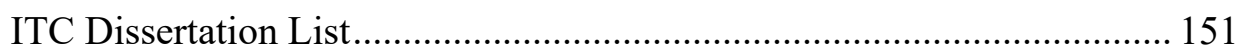




\section{List of figures}

Figure 1.1: Location of the Bavarian Forest National Park, Germany. The satellite image was from the world base map in ArcGIS software (ESRI, Inc., USA)..

Figure 2.1: Canopy spectral reflectance of Asplenium nidus in the visible, NIR and SWIR regions corresponding to LAI values between 0.87 and $6.11 \mathrm{~nm}$. (Source: (Darvishzadeh et al. 2009)).

Figure 2.2: The canopy emissivity spectra in Azalea japonica (a), Buxus sempervirens (b), Euonymus japonicus (c), and Ficus benjamina (d). Changes in canopy emissivity spectra become smaller when LAI values exceed $4\left(\mathrm{~m}^{2} \mathrm{~m}^{-2}\right)$.....

Figure 2.3: The mean canopy emissivity spectra of different plant species with similar LAI value of (a) $1.5\left(\mathrm{~m}^{2} \mathrm{~m}^{-2}\right)$, (b) $2.5\left(\mathrm{~m}^{2} \mathrm{~m}^{-2}\right)$, and (c) $3.5\left(\mathrm{~m}^{2} \mathrm{~m}^{-2}\right)$. Plant species that are not statistically significant from each other are shown in red.

Figure 3.1: The canopy emissivity spectra of four plant species in a thermal infrared region corresponding between $8 \mu \mathrm{m}$ and $14 \mu \mathrm{m}$. .36

Figure 3.2: The relationship between canopy emissivity spectra at individual wavelengths and leaf area index in Azalea japonica (a), Euonymus japonicus (b), Ficus benjamina (c), and Buxus sempervirens (d). The gray lines represent the wavebands at which the canopy emissivity spectra correlated statistically significantly with leaf area index.

Figure 3.3: $2-\mathrm{D}$ correlation plots representing the coefficient of determination $\left(\mathrm{R}^{2}\right)$ between leaf area index and simple difference index for Ficus benjamina (a), Azalea japonica (b), Buxus sempervirens (c), Euonymus japonicus (d), and pooled data (e) calculated from all possible two-waveband combinations between $8 \mu \mathrm{m}$ and $14 \mu \mathrm{m}$....45

Figure 3.4: Scatterplot of measured versus predicted leaf area index for the best narrow band indices calculated from simple difference for Ficus benjamina (a), Azalea japonica (b), Buxus sempervirens (c), Euonymus japonicus (d), and pooled data (e). The optimum wavebands are those reported in Table 3.3.

Figure 3.5: Scatterplot of measured versus estimated leaf area index using entire emissivity spectra in partial least squares regression model from 8-14 $\mu \mathrm{m}$ : Ficus benjamina (a), Azalea japonica (b), Euonymus japonicus (c), Buxus sempervirens (d), and Pooled data (e).

Figure 3.6: Scatterplot of measured versus estimated leaf area index using LMANN training algorithm with 279 wavebands as input data from 8-14 $\mu \mathrm{m}$ : Ficus benjamina (a), Azalea japonica (b), Euonymus japonicus (c), Buxus sempervirens (d), and pooled data (e).

Figure 4.1: Measured leaf area index $\left(\mathrm{m}^{2} \mathrm{~m}^{-2}\right)$ versus measured equivalent water thickness $\left(\mathrm{g} \mathrm{cm}^{2}\right)(\mathrm{a})$, and fuel moisture content $(\%)(\mathrm{b})$, and measured equivalent water thickness versus measured fuel moisture content (c).

Figure 4.2: The $\beta$ coefficients associated with the PLSR model for canopy equivalent water thickness $\left(\mathrm{EWT}_{\mathrm{C}}\right.$ ) (left side diagrams), and canopy fuel moisture content $\left(\mathrm{FMC}_{\mathrm{C}}\right)$ (right side diagrams) for the four species The threshold is based on the corresponding standard deviation of the $\beta$ coefficient. .70

Figure 4.3: Scatterplots of measured versus estimated canopy equivalent water thickness (a), and canopy fuel moisture content (b) for the four species Ficus benjamina, Azalea 
japonica, Euonymus japonicus, Buxus sempervirens, and Pooled data. The blue line represents a first order (linear) regression.

Figure 5.1: Location of the Bavarian Forest National Park, Germany, and the location of the sample plots

Figure 5.2: The relationship between the measured leaf area index and proportion of vegetation cover. The blue line represents a first order (linear) regression.

Figure 5.3: Boxplots are demonstrating the median, lower and upper quartile values of the proportion of vegetation cover calculated using artificial neural networks (ANN), variable atmospherically resistant index values (VARIgreen) and in situ measurements.

Figure 5.4: Scatterplots of measured versus predicted proportion of vegetation cover calculated from artificial neural networks (ANN) (a), and variable atmospherically resistant index values (VARIgreen) (b)....

Figure 5.5: Boxplots and histograms represent the normal distribution of extracted land surface emissivity of 37 plots from TIRS band 10 (a), and band 11 (b), Landsat 8 in the Bavarian Forest National Park

Figure 5.6: The land surface emissivity of plots with different vegetation types and an approximate LAI value of $4 \mathrm{~m}^{2} \mathrm{~m}^{-2}$. .95

Figure 5.7: Scatterplots of in situ measured leaf area index (LAI) and land surface emissivity for 37 plots for Landsat 8 thermal bands. .96

Figure 5.8: Scatterplot of measured versus predicted leaf area index calculated from the simple ratio index (SR).

Figure 5.9: Scatterplot of measured versus estimated leaf area index using different input networks: OLI bands (a), TIRS bands (b), OLI + TIRS bands (c). .98

Figure 6.1: Effects of leaf area index variability on nadir-viewed canopy emissivity in the Buxus sempervirens (a), and Euonymus japonicus (b). 107

Figure 6.2: Changes in canopy emissivity spectra become smaller when LAI values exceed $4.0\left(\mathrm{~m}^{2} \mathrm{~m}^{-2}\right)$ in the Buxus sempervirens (a), and Euonymus japonicus (b)...... 108

Figure 6.3: The relationship between canopy emissivity spectra at individual wavebands and leaf area index. The gray area represents the wavebands that were significantly different.

Figure 6.4: Scatterplots of measured versus estimated canopy equivalent water thickness (left side diagrams), and canopy fuel moisture content (right side diagrams) for the pooled data (a), Ficus benjamina (b), and Azalea japonica (c) using partial least square regression. 113

Figure 6.5: Comparison of the first-order polynomial fit obtained from different approaches for prediction of the proportion of vegetation cover $\left(\mathrm{P}_{\mathrm{V}}\right)$ in the Bavarian forest national park: normalized difference vegetation index $\left(\mathrm{P}_{\mathrm{V}}{ }^{\mathrm{NDVI}}\right)$; variable atmospherically resistant index $\left(\mathrm{P}_{\mathrm{V}}{ }^{\text {VARIgreen }}\right)$; artificial neural networks $\left(\mathrm{P}_{\mathrm{V}}{ }^{\mathrm{ANN}}\right)$.

Figure 6.6: Median, lower and upper quartile values of the proportion of vegetation $\left(\mathrm{P}_{\mathrm{V}}\right)$ calculated using three different methods: normalized difference vegetation index $\left(\mathrm{P}_{\mathrm{V}}{ }^{\mathrm{NDVI}}\right)$; variable atmospherically resistant index $\left(\mathrm{P}_{\mathrm{V}}{ }^{\text {VARIgreen }}\right)$; artificial neural networks $\left(\mathrm{P}_{\mathrm{V}}{ }^{\mathrm{ANN}}\right)$; and in situ measurements. 
Figure 6.7: The land surface emissivity in Norway spruce (Picea abies) over a thermal infrared region corresponding to variation in leaf area index (LAI, $\mathrm{m}^{2} \mathrm{~m}^{-2}$ ). 115

Figure 6.8: Scatterplot of measured versus estimated leaf area index using the artificial neural network and Landsat 8 bands from TIRS sensor (a), OLI sensor (b), and OLI and TIRS sensors (c).

\section{List of tables}

Table 1.1: Classification of the electromagnetic spectrum into various domains.

Table 2.1: Summary statistics of the leaf area index (LAI) measurements for four plant species $(\mathrm{n}=144)$.

Table 2.2: Summary statistics of Two-way ANOVA analyzing canopy emissivity spectra for different species, wavelength, and LAI value. The mean difference is significant at the 0.05 level.

Table 2.3: Summary statistics from One-way ANOVA with Post-hoc Tukey's HSD test of canopy emissivity spectra for four plant species with LAI value binned around 1.5, 2.5 and $3.5\left(\mathrm{~m}^{2} \mathrm{~m}^{-2}\right)$. Where (1) is Azalea japonica, (2) is Buxus sempervirens, (3) is Ficus benjamina, and (4) is Euonymus japonicus.

Table 3.1: Spectral indices used in this paper.

Table 3.2: Summary statistics of the leaf area index (LAI) measurements and emissivity values for four plant species $(n=144)$.

Table 3.4: Performance of partial least squares regression (PLSR), and the important wavebands selected by PLSR model for estimating leaf area index.

Table 3.5: Cross-validated results ( $\mathrm{R}^{2}$ and RMSE) of the estimated leaf area index obtained using neural networks with different inputs (e.g. four plant species), and network structures. $\mathrm{R}^{2} \mathrm{CV}$ and $\mathrm{RMSE}_{\mathrm{CV}}$ represent average results of 1000 random initializations.

Table 4.1: The statistics of the variables measured per plant species. LAI represents the leaf area index $\left(\mathrm{m}^{2} \mathrm{~m}^{-2}\right)$; EWT $\mathrm{C}$ is the canopy equivalent water thickness $\left(\mathrm{g} \mathrm{cm}^{-2}\right)$, EWT is the equivalent water thickness $\left(\mathrm{g} \mathrm{cm}^{-2}\right), \mathrm{FMC}_{\mathrm{C}}$ is the canopy fuel moisture content, and FMC is the fuel moisture content $(\%)$.

Table 4.2: The performance of partial least square regression for estimating canopy equivalent water thickness $\left(\mathrm{EWT}_{\mathrm{C}}, \mathrm{g} \mathrm{cm}^{-2}\right)$, and canopy fuel moisture content $\left(\mathrm{FMC}_{\mathrm{C}}\right)$. $\mathrm{RMSE}_{\mathrm{CV}}$ is the relative cross-validated root mean squared error and $\mathrm{R}^{2}$ is the coefficient of determination.

Table 4.3: Cross-validated results $\left(\mathrm{R}^{2} \mathrm{CV}\right.$ and $\left.\mathrm{RMSE}_{\mathrm{CV}}\right)$ of estimated canopy equivalent water thickness $\left(\mathrm{EWT}_{\mathrm{C}}\right)$ and canopy fuel moisture content $\left(\mathrm{FMC}_{\mathrm{C}}\right)$, obtained using artificial neural networks with different network inputs structures. .72

Table 5.1: The Landsat 8 sensors, the operational land imager (OLI) and the thermal infrared sensor (TIRS) spectral bands and spatial resolution. .86

Table 5.2: Vegetation indices used in this study for the retrieval of leaf area index.....89

Table 5.3: Different input networks for estimation of LAI using artificial neural networks. LSE represents land surface emissivity and was calculated using two thermal 
bands in the thermal infrared region; OLI bands represent reflectance spectra from seven bands in the visible-near infrared and short-wave infrared regions

Table 5.5: Summary of statistics regarding One-way ANOVA with Post-hoc Tukey's HSD test of land surface emissivity (LSE) and vegetation type $(n=37)$. The mean difference is significant at the 0.05 level. .95

Table 5.6: The coefficients of determination $\left(\mathrm{R}^{2}\right)$ for different indices calculated using land surface emissivity (LSE) and leaf area index. $\mathrm{RMSE}_{\mathrm{CV}}$ and $\mathrm{R}^{2} \mathrm{CV}$ are the crossvalidated root mean square error and coefficient of determination between measured and estimated LAI, respectively

Table 6.1: Performance of simple ratio index (SR), partial least squares regression (PLSR), and artificial neural networks (ANN) models for predicting leaf area index. 110 


\section{List of Abbreviation}

\begin{tabular}{ll} 
ANOVA & Analysis of variance \\
ANN & Artificial neural networks \\
BFNP & Bavarian forest national park \\
DMC & Dry matter content \\
DWR & Down-welling radiance \\
EBVs & Essential biodiversity variables \\
EWT & Equivalent water thickness \\
EWT & Canopy equivalent water thickness \\
E & Emissivity \\
FLAASH & Fast line-of-sight atmospheric analysis of spectral hypercubes \\
FMC & Fuel moisture content \\
FMC & Canopy fuel moisture content \\
FTIR & Fourier transform infrared \\
HSD & Honesty significant difference \\
IR & Infrared \\
LAI & Leaf area index \\
LSE & Land surface emissivity \\
LMANN & Levenberg- Marquardt algorithm \\
MATLAB & Matrix Laboratory \\
Max & Maximum \\
MCT & Mercury-Cadmium - Telluride detector \\
Min & Minimum \\
MIR & Mid-infrared \\
MODIS & Moderate resolution imaging spectroradiometer \\
mrad & milliradian \\
MSR & Modified simple ratio \\
MVI & Modified vegetation index \\
ND & Normalized difference index \\
NDVI & Normalized difference vegetation index \\
NDVI & Normalized difference vegetation index thresholds method \\
NIR & Near infrared \\
OLI & Operational land imager sensor \\
PCA & Principal component analysis \\
PCs & Principal components \\
PLSR & Partial least square regression \\
\hline MEd &
\end{tabular}




$\begin{array}{ll}\mathbf{P}_{\mathbf{V}} & \text { Proportion of vegetation cover } \\ \mathbf{R}^{2} & \text { Coefficient of determination } \\ \mathbf{R}^{2} \mathbf{C V} & \text { Coefficient of determination (Cross-validated) } \\ \mathbf{R D I} & \text { Renormalized difference index } \\ \text { RMSE } & \text { Root mean squared error } \\ \text { RMSE }_{\mathbf{C V}} & \text { Root mean squared error (Cross-validated) } \\ \text { ScgANN } & \text { Scaled conjugate gradient algorithm } \\ \text { SD } & \text { Simple difference index } \\ \text { SEBASS } & \text { Spatially enhanced broadband array spectrograph system } \\ \text { SR } & \text { Simple ratio index } \\ \text { SWIR } & \text { Short wave infrared } \\ \text { TIR } & \text { Thermal infrared } \\ \text { TIRS } & \text { Thermal infrared sensor } \\ \text { TOMCAT } & \text { Toolbox for multivariate calibration techniques } \\ \text { VARIgreen } & \text { Variable atmospherically resistant index } \\ \text { VIs } & \text { Vegetation indices } \\ \text { VNIR } & \text { Visible-near infrared }\end{array}$


Chapter 1

General Introduction 


\subsection{Remote sensing of biophysical and biochemical characteristics}

There is an urgency to improve our understanding of terrestrial ecosystem status in a time of rapid climate and land use change. Terrestrial vegetation is a critical component of this ecosystem. Therefore, for its' sustainable management, appropriate knowledge on vegetation status is required (Schmidt et al. 2004). In general, vegetation biophysical and biochemical variables are considered as important indicators of vegetation health and growth status. However, traditional methods to measure these variables are time-consuming, date lagged and costly (Pu et al. 2003).

Remote sensing is an approach which enables observing and acquiring of information about an object or process without making physical contact with them is a practical and an economical means to study vegetation status, and has been recognized as an alternative and efficient method to obtain information about vegetation surfaces and their properties, particularly over large areas (Duro et al. 2007; Stoms and Estes 1993).

The term "Remote Sensing" was initially introduced in the United States in the 1960s by Evelyn L. Pruitt of the U.S. Office of Naval Research (Geography 1965; Pruitt 1970). Before the 1960s, this term used was generally aerial photography. The aerial photographic surveys were started in the middle of the $19^{\text {th }}$ century for collecting data for scientific purposes (Vierling et al. 2006). Subsequently, in recent decades with the proliferation of computers as research tools, the acquisition, processing analyzing remotely sensed data, and digital image processing has become dominant (Asner 1998; Brilis et al. 2001; Goel 2003). Accordingly, during the past couple of decades, remote sensing has rapidly developed as a fast, repetitive, and non-destructive approach to understand ecosystem processes (Kampe et al. 2010; Kimball et al. 2000; Liu et al. 1997), and has been used for ecological studies (Kerr and Ostrovsky 2003; Pettorelli et al. 2005), discrimination of species (Schmidt and Skidmore 2003; Sobhan 2007), assessing vegetation stress (Asner et al. 2004; Li et al. 2005; Suárez et al. 2008), studies of forest dynamics (Broich et al. 2011; Carter and Knapp 2001; Hikosaka and Anten 2012; Poulter and Cramer 2009), quantifying vegetation biophysical parameters (Cho et al. 2007; Darvishzadeh et al. 2008a; Darvishzadeh et al. 2008b; Schlerf et al. 2005) as well as biochemical parameters (Clevers et al. 2010; Kokaly et al. 
2009; Mirzaie et al. 2014; Wang et al. 2015; Zarco-Tejada et al. 2001; ZarcoTejada et al. 2003).

Technological developments in remote sensing instruments allowed the acquisition of remotely sensed data in different spectral domains. The names and the spectral ranges of the various spectral regions are tabulated in Table 1.1 .

Table 1.1: Classification of the electromagnetic spectrum into various domains

\begin{tabular}{ccc}
\hline Name of the spectral domains & Acronyms & Range $(\boldsymbol{\mu m})$ \\
\hline Visible-Near Infrared & VNIR & $0.3-1.0$ \\
Shortwave Infrared & SWIR & $1.0-2.5$ \\
Mid-wave Infrared & MIR & $3-5$ \\
Thermal Infrared & TIR & $8-14$ \\
\hline
\end{tabular}

According to the number of the spectral bands and bands width, remote sensing is classified into three categories consisting of panchromatic, multispectral, and hyperspectral remote sensing (Lillesand et al. 2014). Panchromatic data consists of only a single band with broad wavelength, while multispectral sensors contain few broad spectral bands (i.e. usually between two to ten spectral bands). Since 1972, the inception of the Earth Resource Technology Satellite program (later renamed Landsat) provided the broad range of multispectral data to assess and monitor environmental quality (Campbell and Wynne 2011). In return, hyperspectral sensors typically have a large number of continues narrow spectral bands and have been recognized as powerful tools to identify the surface features and to predict vegetation parameters with the high accuracy (Elvidge and Chen 1995; Schlerf et al. 2005; Sun et al. 2008). In other words, hyperspectral data overcome the restrictions associated with multispectral and panchromatic data for characterizing vegetation variables which were previously impossible to retrieve. For instance, Mutanga and Skidmore (2004) overcame the saturation issue in predicting biomass using narrowband vegetation indices; Darvishzadeh et al. (2008a) estimated leaf area index (LAI) using hyperspectral data and demonstrated the effect of soil background and plant architecture on reflectance spectra; Ceccato et al. (2001) accurately detected water content in terms of equivalent water thickness (EWT) at leaf level using hyperspectral data. 
The VNIR and SWIR regions have been extensively explored using hyperspectral and multispectral data for prediction of vegetation parameters (Cho et al. 2008; Clevers et al. 2010; Darvishzadeh et al. 2009; Mutanga and Skidmore 2004). However, data using VNIR and SWIR are not adequate to describe all the structural and chemical characteristics of vegetation. For instance, the primary absorption features which are linked to certain vegetation components (e.g. water, polysaccharides such as cellulose) and leaf structure variables (e.g. thickness) are only discernable in the MIR and TIR domains (Fabre et al. 2011b; Gerber et al. 2011; Ribeiro da Luz 2006; Ullah 2013).

Among many vegetation parameters that have been studied over these regions, LAI and water content are of particular interest and have received wide attentions. LAI is an important variable for climate and terrestrial ecosystem models, and it is firmly associated with many ecosystem processes such as water balance and evapotranspiration. In addition, LAI has been recently identified as an essential biodiversity variable (EBV) which indicates its importance in various applications (Pettorelli et al. 2016; Skidmore et al. 2015). On the other hand, vegetation water content in terms of fuel moisture content (FMC) and Equivalent water thickness (EWT) is one of the most investigated biochemical variables over VNIR and SWIR regions due to its associations with vegetation physiological status and terrestrial ecosystem conditions (Chuvieco et al. 2004; Claudio et al. 2006; Clevers et al. 2010; Peñuelas et al. 1994). Consequently, these variables were considered further in this study.

\subsection{Thermal remote sensing of vegetation}

Thermal remote sensing resembles the acquisition, processing, and interpretation of image data using the remote sensing but within the thermal infrared region. Sir William Herschel (1800) discovered infrared radiation in sunlight by using a prism and measuring the temperature of each color. $\mathrm{He}$ concluded that there is an invisible form of light beyond the visible part of the prismatic spectrum, what we now call the infrared. TIR remote sensing considers radiation which is emitted in the form of heat from the surface of the object (Kahle 1980; Prakash 2000). In this respect, emissivity is defined as the ratio of the radiance emitted by a body at a particular temperature to the 
radiance emitted by the black body at the same temperature of the body (Becker and Li 1995).

The TIR region has been scarcely perused for vegetation studies. Over two decades have passed since Salisbury et al. (1994) anticipated that at the end of $20^{\text {th }}$ century the Earth observing system would deliver wide-reaching TIR data. Still, we have very little information as regards to the emissivity of plants particularly at canopy level. Estimation of biochemical vegetation variables at canopy level in the TIR region has been hardly examined (Gonzalez-Dugo et al. 2013), while retrieval of biophysical parameters at canopy level has not been considered using emissivity spectra in the TIR domain. Understanding the TIR canopy spectra will become gradually important, and ultimately may enable new types of remote sensing observations over vegetation canopies.

The little attention and some degree misconception that have been given to the TIR data may be due to several issues. First, relatively few laboratories have access to instruments that enable measurements of the spectra over the TIR domain with high signal to noise ratio which is extensively essential for using TIR spectral in vegetation remote sensing studies (Kirkland et al. 2002; Ribeiro da Luz and Crowley 2010), and also the spatial resolution of the most TIR data is observed as being too coarse (e.g. satellite thermal data). The achievement in applying infrared technology to remote sensing only has been made possible through the advanced progress of high-performance infrared sensors over the last six decades (Rogalski 2012). Second, emissivity spectral variations in plants are subtle and complicated (Ribeiro da Luz and Crowley 2010); and third, TIR data is little comprehended from theoretical and applications prospects within the ecological community and in contrast, the details of plant physiology and organic chemistry in association with plants spectral features at the TIR region are unknown to many remote sensing experts (Quattrochi and Luvall 1999).

Few studies have evaluated the potential of the TIR for characterizing vegetation. For instance, in the middle of the last century, Gates and Tantraporn (1952) measured leaf reflectance spectra of shrubs and numerous deciduous trees between $1 \mu \mathrm{m}$ and $25 \mu \mathrm{m}$ wavelengths. In preliminary studies, Salisbury and Milton (1988) used TIR data to investigate the reflectance spectra of leaves from different plant species over $2.5 \mu \mathrm{m}$ and $13.5 \mu \mathrm{m}$ domain and showed that deciduous species have unique reflectance features; Rubio et 
al. (1997) measured the emissivity of 35 vegetation species in the wavelengths between $8 \mu \mathrm{m}$ and $14 \mu \mathrm{m}$ and concluded that the vegetation shows a near grey body behavior. During the past decade, only a few studies focused on spectral reflectance as well as emissivity features in vegetation using TIR hyperspectral data. For instance, Ribeiro da Luz and Crowley (2010) and Ullah et al. (2012a) investigated the identification of plant species using emissivity in the TIR region. Only a few researchers opined that the TIR region contains relevant information regarding the biochemical concentration in leaves (Fabre et al. 2011b; Gerber et al. 2011; Ribeiro da Luz and Crowley 2007, 2010; Ullah et al. 2012a). In this regard recently, a few studies focused on estimation of water content using emissivity spectra of plants at leaf level (Ullah et al. 2013; Ullah et al. 2012b; Ullah et al. 2014). Buitrago et al. (2016), investigated the influence of water content in plant species on emissivity spectra and concluded that changes in emissivity spectra might be associated with the variations in the cuticle thickness and conceivably the structure of cuticle. In addition, TIR data have found to be valuable for other applications including measuring evapotranspiration, water consumption in vegetation (Lillesand et al. 2014), and estimation of energy fluxes (Frey and Parlow 2012).

To our knowledge, most of the studies mentioned above in this domain (i.e. TIR) have been conducted at the leaf level, and little consideration has been given to the assessment of emissivity features as well as plant properties at the canopy level. Furthermore, the majority of studies which have focused at the leaf level, have collected the leaves in the field and transported to the laboratory for spectral or biochemical properties measurements. Despite the fact that in situ measurements at the leaf level are crucial for validation and also depends on the goals of the research studies, but this is often impractical due to the instability of environmental status (e.g. weather and temperature), and inaccessibility for portable spectral equipment. Another limitation of leaf level measurements is that leaf physiology, as well as reflectance or emissivity spectral properties, changes over the relatively short period (Foley et al. 2006). Moreover, the demand for monitoring of ecosystems over a large area is increased due to the climate changes and habitat degradation. This would be only practical by using remotely sensed data provided by airborne and space born platforms. As a consequence, ecologists and forest managers have become more interested in vegetation studies at canopy level and have a desire 
to improve their understanding of the ecosystem status because a plant canopy is a particular place of the biophysical and biochemical process in ecosystems (Asner et al. 2003).

In this study, we have focused on the retrieval of vegetation properties at the canopy level using thermal remote sensing measurements. Since the relation between laboratory measurements at the canopy level and satellite images using thermal remote sensing data is lacking, the potential of laboratory-based TIR emissivity spectra as well as TIR satellite imagery (i.e. Landsat 8 , thermal infrared sensor (TIRS) bands) are investigated for prediction of vegetation biochemical and particularly biophysical parameters at the canopy level. The MIDAC illuminator Fourier Transform Infrared (FTIR) spectrometer as a laboratory and also point based field spectrometer which measure the radiance spectra of plants species in hundred spectral bands were utilized first under controlled laboratory conditions, next the Landsat 8 bands particularly TIRS bands which are more dependable to the prediction of surface emissivity were employed to estimate land surface emissivity and further estimate vegetation properties.

\subsection{Research objectives}

The primary aims of this study were to:

1) Investigate the effects of variation of LAI on emissivity spectra at the canopy level using TIR hyperspectral remote sensing data.

2) Evaluate the retrieval of LAI by applying univariate and multivariate techniques using TIR hyperspectral remote sensing data.

3) Investigate the potential of canopy emissivity for estimating FMC and EWT as mass-based and area-based biochemical variables respectively using TIR hyperspectral data at the canopy level.

4) Investigate whether the established relations between LAI and land surface emissivity at the laboratory level can be extended to satellite level using thermal data on a large scale.

\subsection{The study area}

Considering the research objectives and the availability of TIR hyperspectral data Chapters 2, 3, and 4 benefited from laboratory experimental data to retrieve LAI, FMC, and EWT at the canopy level by means of TIR 
hyperspectral measurements using a portable MIDAC FTIR spectrometer (Model M4401-F; MIDAC Corporation, CA, USA) under controlled laboratory conditions where the walls, ceiling, and ground were covered with a black plastic material of known emissivity.

However, the capability and upscaling of laboratory experiments are always faced with the query of whether such studies are extendable to natural ecosystems. In this respect, to proof the concept of predicting vegetation characteristics using TIR data, in Chapter 5, the satellite data were obtained from Landsat 8 for the mixed mountain forest of Bavarian Forest National Park (BFNP) which is located in a southeastern part of Germany along the border of the Czech Republic (49 $3^{\circ} 19^{\prime \prime} \mathrm{N}, 13^{\circ} 12^{\prime} 9^{\prime \prime}$ E) (Figure 1.1). Elevation in BFNP ranges from $600 \mathrm{~m}$ to $1453 \mathrm{~m}$ above sea level. The climate of the region is temperate, and precipitation varies from 1200 to 1800 $\mathrm{mm} /$ year and, minimum average annual temperature between $3{ }^{\circ} \mathrm{C}$ and $6^{\circ} \mathrm{C}$. In general, the dominant tree species in the BFNP consists European beech (24.5\%), Norway spruce (67\%), and Fir (2.6\%) (Heurich et al. 2010).

\subsection{Thesis outline}

This thesis comprises of an entire six chapters, including a general introduction, four core chapters, and a synthesis. Each core chapter has been provided as a standalone research paper that has been published in or submitted to the peer-review ISI journals. The structure of the chapters is as follows.

Chapter 1 presents the research background, research objectives, and also describes the study area and outlines of the thesis.

Chapter 2 deals with the effect of LAI on canopy emissivity spectra in the TIR domain from different species under controlled laboratory conditions and at the nadir position. 


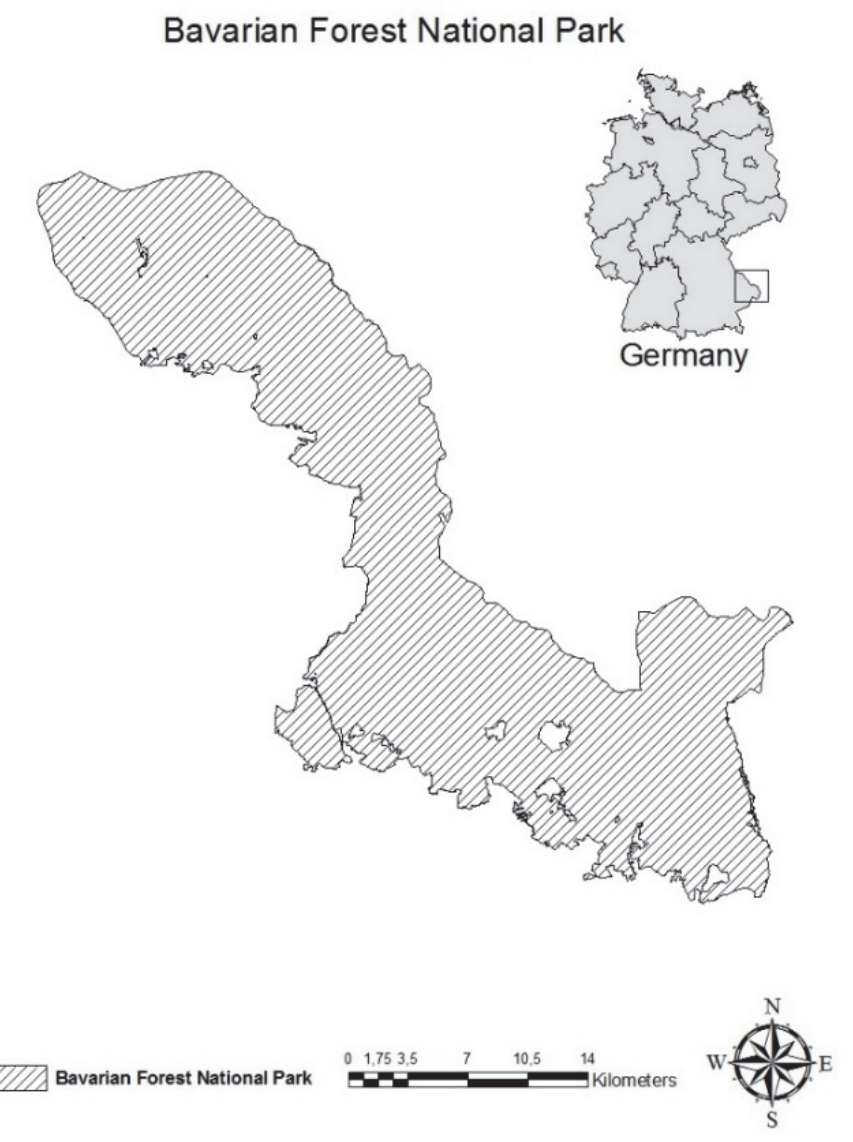

Figure 1.1: Location of the Bavarian Forest National Park, Germany. The satellite image was from the world base map in ArcGIS software (ESRI, Inc., USA).

Chapter 3 evaluates the retrieval of LAI using TIR hyperspectral data which were obtained under controlled laboratory conditions for four plant species and examined the utility of different univariate and multivariate methods to predicting LAI.

Chapter 4 applies TIR hyperspectral data for predicting FMC as a mass-based and EWT as area based water indicators at the canopy level.

Chapter 5 evaluates the estimation of LAI from TIR satellite imagery data which acquired at the same time as the field campaign held in BFNP as temperate mixed forest using statistical and machine learning approaches. 
Chapter 6 provides an overview of the significant research findings of the thesis. The contributions of the undertaken studies in this thesis in the realm of EBV assessment are discussed. Further research directions and some recommendation are also proposed. 


\section{Chapter 2}

\section{Measuring the Response of Canopy Emissivity Spectra to Leaf Area Index Variation using Thermal Hyperspectral Data*}

\footnotetext{
* This chapter is based on:

Neinavaz, E., Darvishzadeh, R., Skidmore, A.K., Groen, and T.A. 2016. Measuring the Response of Canopy Emissivity Spectra to Leaf Area Index Variation using Thermal Hyperspectral Data. International Journal of Applied Earth Observation and Geoinformation 50.40-47.

Neinavaz, E., Darvishzadeh, R., Skidmore, A.K., Groen, and T.A., Hecker, C. Estimation of Leaf Area Index from Hyperspectral Thermal Data. $9^{\text {th }}$ EARSeL SIG Imaging Spectroscopy Workshop, 14-16 April 2015, Luxemburg.
} 


\begin{abstract}
One of the plant biophysical factors affecting the canopy spectral reflectance of plants in the optical domain to receive research attention in recent decades is leaf area index (LAI). Although it is expected that the value of LAI affects the emission of radiation, it not known how. To our knowledge, the effect of LAI on plant canopy emissivity spectra has not yet been investigated in the thermal infrared region (TIR 8-14 $\mu \mathrm{m}$ ). The overall aim of this study was to demonstrate the effect of LAI on canopy emissivity spectra of different species at the nadir position. The 279 spectral wavebands in the TIR domain were measured under controlled laboratory condition using a MIDAC spectrometer for four plant species. The corresponding LAI of each measurement was destructively calculated. We found a positive correlation between canopy emissivity spectra at various LAI values, indicating that emissivity increases concomitantly with LAI value. The canopy emissivity spectra of the four species were found to be statistically different at various wavebands even when the LAI values of the species were similar. It seems that other biophysical or biochemical factors also contribute to canopy emissivity spectra: this merits further investigation. We not only quantify the role of LAI on canopy emissivity spectra for the first time but also demonstrate the potential of using hyperspectral thermal data to estimate LAI of plant species.
\end{abstract}




\subsection{Introduction}

In recent decades, vegetation reflectance spectra and their features in the visible/near-infrared (VNIR, 0.3-1.0 $\mu \mathrm{m}$ ) and short-wave infrared (SWIR, 1.0-2.5 $\mu \mathrm{m})$ regions of the electromagnetic spectrum have been widely investigated. Consequently, meaningful relationships have been established between reflectance spectra and vegetation biophysical, and biochemical properties at leaf, canopy, and landscape levels (Asner 1998). Despite the growing importance of remote sensing for vegetation studies, it is not fully understood how the emissivity spectra interact with biophysical and biochemical properties of vegetation in the thermal infrared region (TIR 8-14 $\mu \mathrm{m})$. TIR hyperspectral data is important for earth observation such as geological remote sensing studies, due to the strong response of minerals (e.g. quartz) in this domain (Van der Meer et al. 2012). Additionally, TIR data is particularly important for investigating vegetation phenomena in which temperature plays a critical role (e.g. photosynthesis, and transpiration) (Lindroth et al. 2008).

Leaf area index (LAI) is a dimensionless variable, defined as the one-sided leaf area $\left(\mathrm{m}^{2}\right)$ per unit of horizontal surface area $\left(\mathrm{m}^{2}\right)$ (Watson 1947), and it is a critical input for climate and large-scale ecosystem models (Zheng and Moskal 2009). In addition, the LAI is an important biophysical parameter of vegetation that exhibits a primary control on the plant energy balance, transpiration, respiration, and gas exchanges (e.g. uptake of $\mathrm{CO}_{2}$ and $\mathrm{H}_{2} \mathrm{O}$ by the canopy) (Running and Coughlan 1988). Previous studies have revealed the importance of LAI in ecological and remote sensing studies. For instance, process-based ecosystem simulations are often required to produce quantitative analyses of productivity; in this regard, LAI is a key input parameter to such models (Liu et al. 1999; Liu et al. 1997; Matsushita et al. 2004). Also, LAI is indispensable for scaling between leaf and canopy measurements of biochemical variables and biosphere-atmospheric exchange (e.g. water vapor, $\mathrm{CO}_{2}$ conductance, and flux) at the global scale (Asner et al. 2003). In addition, long-term monitoring of LAI can provide critical information on climate impacts on ecosystems (Zheng and Moskal 2009). LAI can explain the differences between photosynthesis and respiration in different ecosystems as well as how photosynthesis varies under different light levels and leaf nitrogen concentrations in the area of low and high LAI respectively (Boegh et al. 2002). 
Emissivity spectra depend on leaf surface temperature (Becker and Li 1990) and are part of the radiation term in the energy budget of the leaves (Gates 2012). The temperature of a canopy surface depends on the balance between incoming solar energy and energy loss. Leaves absorb a certain fraction of the incident radiation with this energy, dividing it over three outgoing streams: re-radiation, convective heat exchange with the air, and evaporation or transpiration (Gates 2012). A large proportion of these outgoing streams is devoted to convective heat exchange with the air. In other words, most of the incoming solar energy in plants is transformed and lost as heat (McKinney and Schoch 2003). It has been shown that regarding physiology and ecology; plants are able to regulate their energy balance through their leaf surfaces (Delrot et al. 2010). It has been demonstrated by Drake et al. (1970) that transpiration has an important role to stabilization of the leaf temperature, particularly at high environmental temperature through the cooling role of transpiration. In this respect, Vertessy et al. (1995) showed that the relation between transpiration and leaf area is almost linear. Further, LAI is related to the efficiency of canopy evaporation value (van den Hurk et al. 2003). Brutsaert (2013), has demonstrated that high LAI values are strongly correlated with the efficiency of canopy evaporation and account for the majority of the existent energy, applied for evapotranspiration. Therefore, it can be realized that LAI variability affects canopy evaporation and transpiration.

Previous studies have investigated the effect of varying LAI values on canopy reflectance and have demonstrated that with rising LAI the canopy reflectance spectra increases in particular in NIR region (Asner 1998; Darvishzadeh et al. 2009). As can be observed from Figure 2.1, the variation of LAI has an influence on canopy reflectance signatures, with the most pronounced impact in the NIR domain $(750 \mathrm{~nm}$ to $1350 \mathrm{~nm})$. Moreover, as LAI rises within a canopy, an obvious deepening of the two water absorption features within the NIR region located at $1000 \mathrm{~nm}$ and $1200 \mathrm{~nm}$ can be observed in the reflectance spectra. To date, our knowledge regarding the canopy emissivity spectra and its relation to LAI in the TIR region has been limited by mainly technical problems (Ribeiro da Luz and Crowley 2007).

Recently, thermal hyperspectral devices have improved: new thermal infrared sensors discern TIR spectral features at higher spectral resolution (e.g. the MIDAC illuminator Fourier Transform Infrared (FTIR) spectrometer). 
Reviewing the literature revealed that most vegetation studies using TIR hyperspectral data have focused at the leaf level (Buitrago et al. 2016; Ullah et al. 2013; Ullah et al. 2014), and only a few studies have addressed TIR hyperspectral data at canopy level (Ribeiro da Luz and Crowley 2010; Sepulcre-Cantó et al. 2006). In preliminary studies, Salisbury and Milton (1988) were among the first to use thermal data to investigate the reflectance spectra of different plant species at leaf level in the $2.5-13.5 \mu \mathrm{m}$ region. They showed that deciduous species have unique reflectance features. Ullah et al. (2012a), studied the leaf emissivity spectra for different species and demonstrated that vegetation has specific characteristic emissivity signatures at leaf level.

The above literature revealed that leaves are not opaque and featureless in the TIR domain and that hyperspectral thermal remotely sensing of vegetation is an area in which there is still much to explore. No studies have focused on the biophysical properties of vegetation and their influence on emissivity spectra of canopies. Here, for the first time, we use TIR hyperspectral data to evaluate changes in emissivity spectral measurements under different values of LAI measured for structurally different species and under controlled laboratory conditions. Our study had two principal objectives: (1) to measure the response of canopy emissivity spectra to LAI variation and (2) to explore the canopy emissivity spectra of different plant species with the same LAI.

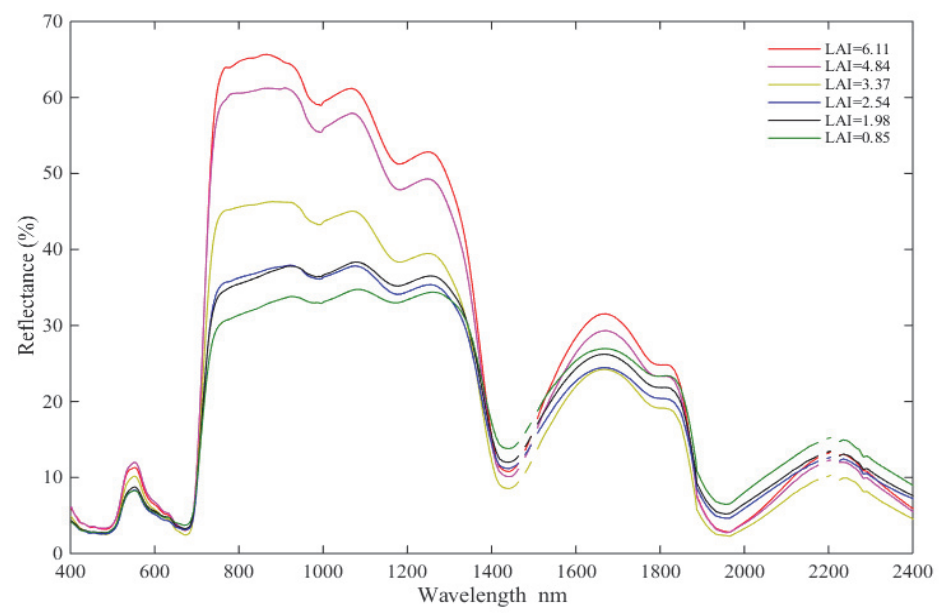

Figure 2.1: Canopy spectral reflectance of Asplenium nidus in the visible, NIR and SWIR regions corresponding to LAI values between 0.87 and $6.11 \mathrm{~nm}$. (Source: (Darvishzadeh et al. 2009)). 


\subsection{Materials and methods}

\subsubsection{LAI measurements}

In the present study, four different plant species were selected: Azalea japonica, an evergreen flowering shrub with ovate leaves about $2-3 \mathrm{~cm}$ long $(\mathrm{n}=10)$; Buxus sempervirens, an evergreen shrub with oval leaves about 1.5$3 \mathrm{~cm}$ long and $0.5-1.3 \mathrm{~cm}$ wide (n=10); Euonymus japonicus, an evergreen shrub with oval leaves about $3 \mathrm{~cm}$ to $7 \mathrm{~cm}$ long, with finely serrated margins $(\mathrm{n}=11)$; and Ficus benjamina, which has glossy, oval leaves about $6-13 \mathrm{~cm}$ long, with an acuminate tip $(\mathrm{n}=6)$. To create variation in LAI and corresponding emissivity measurements, leaves from different layers and on the inner side of the canopy were randomly removed in 3-4 consecutive steps (depending on the plant size), hence after each removal phase, the total LAI value of the canopy were lowered. These harvesting steps were carried out on each sampled species within a few hours to minimize possible changes in the plant's physiological status such as stomatal conductance and to exclude the possible interference of any physiological processes. The areas of harvested leaves were measured using the LI-3000C portable leaf area meter (LICOR, NE, USA) that was regularly calibrated. To calculate the LAI $\left(\mathrm{m}^{2} \mathrm{~m}^{-2}\right)$, the measured surface areas of the leaves $\left(\mathrm{m}^{2}\right)$ were divided by the corresponding ground area of the canopy $\left(\mathrm{m}^{2}\right)$. The dataset includes 37 plants, resulting in 144 LAI measurements, as the plants were destructively sampled for LAI.

\subsubsection{Laboratory condition}

To create optimal measurement conditions, and reduce any possible sources of error due to the changes in atmospheric conditions or temperature, the measurements were carried out under controlled laboratory conditions where the walls, ceiling, and ground were coated with a black material (Avis Aqua Blackboard Black) and plastic of known emissivity. The traditional procedure when measuring emissivity is to heat samples (e.g. geological samples) to a temperature above ambient condition to create a thermal contrast (Ribeiro da Luz and Crowley, 2007; Salisbury, 1998). However, such treatment stresses plant samples, so instead, we reduced the lab temperature to $10^{\circ} \mathrm{C}$ in order to generate a suitable thermal contrast with the plants, which were at a higher room temperature. For this, plants were kept outside the laboratory at an ambient room temperature of $20^{\circ} \mathrm{C}$ and were briefly individually transferred 
to the cool room in order to make the thermal measurements, and then were returned to normal room temperature. In this experiment, the background soil was covered with black plastic of known emissivity to minimize possible effects of soil. We measured the emissivity spectra of the black plastic using a BRUKER Vertex 70 laboratory FTIR spectrometer. The black plastic had a very low emissivity so could be assumed to cause minimal interference with the thermal radiance measurements of the samples.

\subsubsection{Canopy spectroscopic measurement}

\subsubsection{Thermal infrared emission spectroscopy}

The radiance spectra were measured using a portable MIDAC FTIR spectrometer (Model M4401-F; MIDAC Corporation, CA, USA). The MIDAC configuration enables the measurement of radiance spectra within the spectral range of 2.5-20 $\mu \mathrm{m}$ with an adjustable spectral resolution of 32 $0.5 \mathrm{~cm}^{-1}$ (Eisele et al. 2015). The MIDAC has a liquid-nitrogen-cooled Mercury-Cadmium-Telluride (MCT) detector and customized foreoptics that consist of a flat folding mirror on a rotational axis, which allows measurements of two blackbodies (hot and cold), for calibrating and measuring each sample. The MIDAC's folding mirror was kept at nadir position above the samples. The MIDAC's field of view (using a cut-off of $5 \%$ of the maximum responsivity) has a starting diameter of $53 \mathrm{~mm}$ at the folding mirror and spreads with about $18 \mathrm{mrad}$. Since the amount of thermal emission varies according to the distance between the sample and the sensor (Ribeiro da Luz and Crowley 2007), and also to reduce atmospheric attenuation (Korb et al. 1996), measurements were made with a fixed vertical distance between sensor and sample.

\subsection{Radiometric calibration and down-welling radiance measurements}

The emissivity spectra of plant canopies were obtained using a series of FTIR measurements performed in the following order: radiance measurements of the hot blackbody, radiance measurement of the cold blackbody, radiance measurement of the sample (i.e. the plant canopy), and finally, radiance measurements of a highly diffuse reflecting gold plate (Infragold $\AA$ ). For instrument radiance calibration, two individual blackbodies were used. The temperatures of the hot and cold blackbodies were regularly checked between 
measurements of each sample. The cold blackbody temperature was set just below the ambient temperature, at $5^{\circ} \mathrm{C}$ (Korb et al. 1996). The hot blackbody temperature was set above the sample temperature, at $30^{\circ} \mathrm{C}$ (Hori et al. 2006; Salvaggio and Miller 2001). Details of the radiometric calibration that was applied to the measurements of these blackbodies radiances can be found in Hook and Kahle (1996). A diffuse reflecting gold plate with an emissivity of $\sim 0.04$ was used to measure down-welling radiance (DWR) in order to correct radiance measurements and determine any significant influence of laboratory background emissions (Eisele et al. 2015). The infragold plate was placed directly under the MIDAC sensor at the same distance as the sample. The temperatures of the sample, infragold plate, and lab were frequently monitored before and after each measurement, using thermistors (FLUKE 51 II Thermometer and Precision IR Thermometer) to detect any possible changes in temperature that could affect the measurements, as such changes in temperature could disturb the thermal contrast and result in over- or underestimation of the emissivity values. The measurement series were taken within five minutes to minimize possible temperature drift of the instrument, physiological changes in the plants, and fluctuations in laboratory temperature (Hori et al. 2006).

\subsection{Canopy radiance measurements}

The radiance spectra of the plant canopies were measured between wavelengths of 2.5-20 $\mu \mathrm{m}$ with a resolution of $2 \mathrm{~cm}^{-1}$. An average of 32 scans was observed for each sample (measurement). The canopy emissivity measurements included 279 wavebands between $8 \mu \mathrm{m}$ and $14 \mu \mathrm{m}$ regions. Measurements outside this range had the very low signal strength and therefore were excluded from further analysis. After each set of measurements, the canopy was rotated $90^{\circ}$ clockwise. The final corresponding canopy emissivity spectra of each sample (for a particular LAI value), was then calculated from the average of four sets of measurements (covering $360^{\circ}$ ). The position of the MIDAC sensor above the canopy was kept constant. In total, $576(4 \times 144)$ canopy radiance measurements were obtained for the four plant species (Table 2.1). 
Table 2.1: Summary statistics of the leaf area index (LAI) measurements for four plant species $(n=144)$.

\begin{tabular}{ccccc}
\hline \multirow{2}{*}{ Species name } & \multicolumn{4}{c}{ LAI $\left(\mathbf{m}^{\mathbf{2}} \mathbf{~ m}^{-\mathbf{2}}\right)$} \\
\cline { 2 - 5 } Mean & Max & Min & Sample size \\
\hline Bulea japonica & 1.57 & 3.35 & 0.60 & 30 \\
Euxus sempervirens & 4.54 & 9.80 & 1.17 & 40 \\
Ficus benjamina & 3.60 & 8.36 & 1.04 & 44 \\
\hline Pooled data & 3.25 & 9.80 & 0.60 & 144 \\
\hline
\end{tabular}

\subsubsection{Data processing and analysis}

Spectral emissivity of the plants was calculated from their absolute radiance using the following equation (Korb et al. 1996),

$\varepsilon_{\text {sam }}(\lambda)=\frac{\mathrm{L}_{\text {sam }}(\lambda)-\mathrm{L}_{\text {DWR }}(\lambda)}{\mathrm{B}\left(\lambda, \mathrm{T}_{\text {sam }}\right)-\mathrm{L}_{\mathrm{DWR}}(\lambda)}$

where $\varepsilon_{\text {sam }}(\lambda)$ denotes the directional emissivity of the sample at the wavelength $\lambda, \mathrm{L}_{\text {sam }}(\lambda)$ is spectral radiance from the target, $\mathrm{T}_{\text {sam }}$ is the actual physical temperature of the sample, $B\left(\lambda, T_{\text {sam }}\right)$ is the Planck function at the wavelength $\lambda$ and sample temperature, and $L_{D W R}(\lambda)$ is total spectral DWR from the hemisphere above the sample. To retrieve canopy surface emissivity, the information regarding precise surface temperature $\mathrm{T}_{\text {sam }}$ at the time of the measurement is essential. Therefore, despite measuring canopy temperature before and after each measurement, the blackbody fit method was used to estimate the exact sample temperature value at the time of measurement. The details about the blackbody fit method can be found in Kahle and Alley (1992) and Salvaggio and Miller (2001). A Savitzky and Golay (1964) filter with a frame size of 15 data points and second- degree polynomial was used to reduce the noise of the canopy emissivity spectra (Savitzky and Golay 1964). Data were analyzed and processed using MATLAB R2013b (Mathwork, Inc).

\subsubsection{Statistical analyses}

Three statistical tests were used in this study: one-way ANOVA, two-way ANOVA, and principal component analysis (PCA). A two-way analysis of variance (ANOVA) was performed to ascertain the effects of 1) variation in LAI values and the wavelength values across $8-14 \mu \mathrm{m}$ (i.e. independent variables) and 2) the interaction between these independent variables, on 
canopy emissivity (the continuous dependent variable). For all species, LAI values were binned in three common classes of $1.5,2.5$ and $3.5\left(\mathrm{~m}^{2} \mathrm{~m}^{-2}\right)$. Also, wavelengths from $8 \mu \mathrm{m}$ to $14 \mu \mathrm{m}$ were binned into six categories ranging from $8 \mu \mathrm{m}$ to $8.99 \mu \mathrm{m}, 9-9.99 \mu \mathrm{m}, 10-10.99 \mu \mathrm{m}, 11-11.99 \mu \mathrm{m}, 12-12.99$ $\mu \mathrm{m}$, and $13-13.99 \mu \mathrm{m}$. We examined whether the mean emissivity at different LAI values or waveband ranges were statistically different and whether there was an interaction between these variables (i.e. LAI and wavelength). In addition, a one-way ANOVA was used to explore whether the mean emissivity of different species with the same LAI value is significantly different. Tukey's honestly significant difference (HSD) test was used as a post-hoc test, to determine the statistical significance of differences between pairs of species and to examine whether mean emissivity in different species with the same LAI value is statistically different.

In addition, Principal Component Analysis (PCA) was used as data reduction technique to determine the significant wavebands for discriminating between plant species with similar LAI values among the 279 wavebands in the TIR region that were studied. PCA is a popular multivariate statistical technique introduced by Pearson (1901) and refined by Hotelling (1933). It has been successfully used in many remote sensing studies as a data reduction approach (Chen et al. 2014; Du and Fowler 2007; Hirosawa et al. 1996; Holden and LeDrew 1998; Tsai et al. 2007). In our analysis, wavebands that have the highest factor loadings on the selected principal components (PCs) have the greatest variation in emissivity between species with similar LAI values and have high information content, due to the significant contribution to the selected PCs.

\subsection{Results}

\subsubsection{Statistical analysis}

The experimental setup ensured a broad range of emissivity spectra at the canopy level and a large range of LAI values. In total, 144 samples were studied, with LAI values varying between $0.60\left(\mathrm{~m}^{2} \mathrm{~m}^{-2}\right)$ in Azalea japonica and $9.80\left(\mathrm{~m}^{2} \mathrm{~m}^{-2}\right)$ in Buxus sempervirens (Table 2.1). In Figure 2.2, canopy emissivity spectra of various plant species are plotted separately, based on different LAI values. It can be seen that LAI and thermal emissivity are positively correlated, in agreement with the positive correlations found 
between LAI and emissivity. As can be seen from Figure 2.2, for all species the emissivity increases in all wavelengths across $8-14 \mu \mathrm{m}$ parts of the electromagnetic spectrum. However, this increment is more pronounced above $9 \mu \mathrm{m}$, and the wavelength in the $8-9 \mu \mathrm{m}$ portion of the electromagnetic spectrum, which is moderately responsive to LAI increases. Three of the measured species, namely, Buxus sempervirens, Euonymus japonicus, and Ficus benjamina, had high LAI values, with maxima above 3.5. Therefore, we further investigated how the change in emissivity spectra responded to higher LAI values. Plots of the emissivity spectra for these species for higher values (Figure $2.2 \mathrm{~b}-\mathrm{d}$ ) show that the difference in emissivity spectra of LAI values between 1.0 and $3.5\left(\mathrm{~m}^{2} \mathrm{~m}^{-2}\right)$ is quite distinct from all four species. For the LAI values larger than $4.0\left(\mathrm{~m}^{2} \mathrm{~m}^{-2}\right)$, the differences in canopy emissivity spectra value become less pronounced and at some wavelengths seem to be saturated.

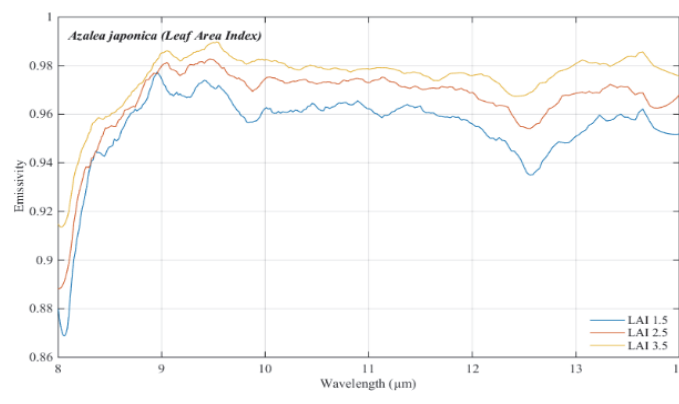

(a)

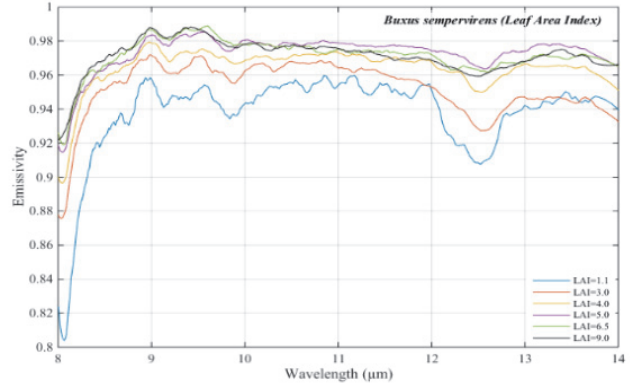

(b) 


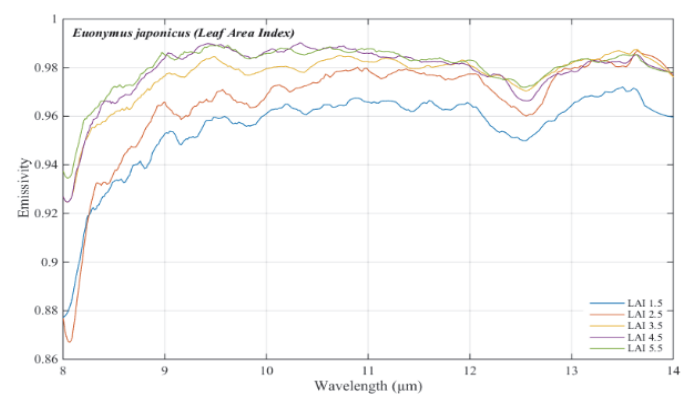

(c)

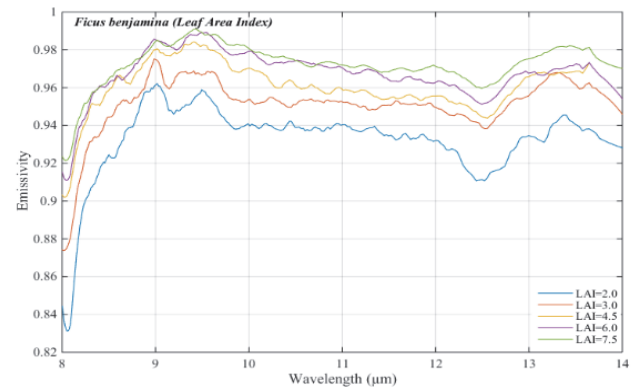

(d)

Figure 2.2: The canopy emissivity spectra in Azalea japonica (a), Buxus sempervirens (b), Euonymus japonicus (c), and Ficus benjamina (d). Changes in canopy emissivity spectra become smaller when LAI values exceed $4\left(\mathrm{~m}^{2} \mathrm{~m}^{-2}\right)$.

The canopy emissivity spectra at several wavelengths reached saturation at a different level, depending on the plant species. For instance, emissivity reaches saturation level in Euonymus japonicus and Buxus sempervirens when LAI exceeds 4.5 and $4\left(\mathrm{~m}^{2} \mathrm{~m}^{-2}\right)$ respectively, while in Ficus benjamina, at wavelengths between $8 \mu \mathrm{m}$ and $9 \mu \mathrm{m}$ saturation is only observed when LAI exceeds $6.5\left(\mathrm{~m}^{2} \mathrm{~m}^{-2}\right)$. The two-way ANOVA results are presented in Table 2.2. The results showed that there was an interaction between wavelength and LAI values for all species. In other words, it seems that the effect on the canopy emissivity spectra of changing LAI values depends on the specific part of the spectrum. 
Table 2.2: Summary statistics of Two-way ANOVA analyzing canopy emissivity spectra for different species, wavelength, and LAI value. The mean difference is significant at the 0.05 level.

\begin{tabular}{cccc}
\hline Species & Source & F statistics & p-value \\
\hline \multirow{3}{*}{ Azalea japonica } & LAI & 79.39 & $<0.001$ \\
& Wavelength & 217.27 & $<0.001$ \\
& LAI $\times$ Wavelength & 4.420 & $<0.001$ \\
\hline \multirow{2}{*}{ Buxus sempervirens } & LAI & 76.07 & $<0.001$ \\
& Wavelength & 218.26 & $<0.001$ \\
& LAI $\times$ Wavelength & 4.54 & 0.004 \\
\hline \multirow{2}{*}{ Euonymus } & LAI & 51.73 & $<0.001$ \\
japonicus & Wavelength & 236.90 & $<0.001$ \\
& LAI $\times$ Wavelength & 9.26 & $<0.001$ \\
\hline \multirow{2}{*}{ Ficus benjamina } & LAI & 134.05 & $<0.001$ \\
& Wavelength & 128.39 & $<0.001$ \\
\hline
\end{tabular}

As can be seen from Figure 2.3, the emissivity spectra and absorption features vary among plant species with similar LAI values. The one-way ANOVA shows significant differences at $p$-value $<0.05$ among all species ([LAI $=1.5$, $F(3,1112)=44.63, \mathrm{MSE}=0.01, p$-value $=0.00],[\mathrm{LAI}=2.5, F(3,1112)=$ $36.29, \mathrm{MSE}=0.01, p$-value $=0.00]$, and $[\mathrm{LAI}=3.5, F(3,1112)=18.82$, MSE $=0.00, p$-value $=0.00]$ ), showing that the mean emissivity of at least one pair of species was statistically different from the others. In addition, the results show that at a similar LAI, the emissivity spectra of the different plant species are mainly different between wavelengths $9-11 \mu \mathrm{m}$ (Figure 2.3). The Tukey's HSD test (Table 2.3) for the six possible pair combinations for the four species showed that the mean canopy emissivity spectra of all species with a similar LAI of $1.5\left(\mathrm{~m}^{2} \mathrm{~m}^{-2}\right)$ were significantly different at the $p$-value $<0.05$ level. However, not all comparisons were statistically significant for LAI values of 2.5 and $3.5\left(\mathrm{~m}^{2} \mathrm{~m}^{-2}\right)$. At a LAI value of $2.5\left(\mathrm{~m}^{2} \mathrm{~m}^{-2}\right)$, there was no statistically significant difference between Azalea japonica and Euonymus japonicus in the mean score for the canopy emissivity spectra. Neither were there 
significant differences between Azalea japonica and Ficus benjamina with a LAI value of $3.5\left(\mathrm{~m}^{2} \mathrm{~m}^{-2}\right)$.

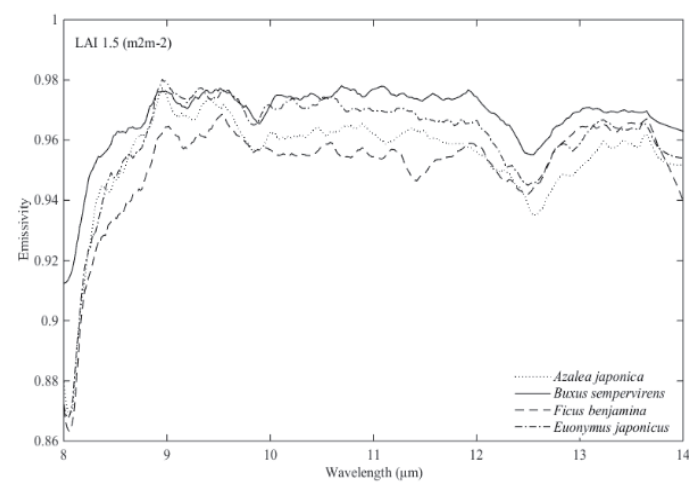

(a)

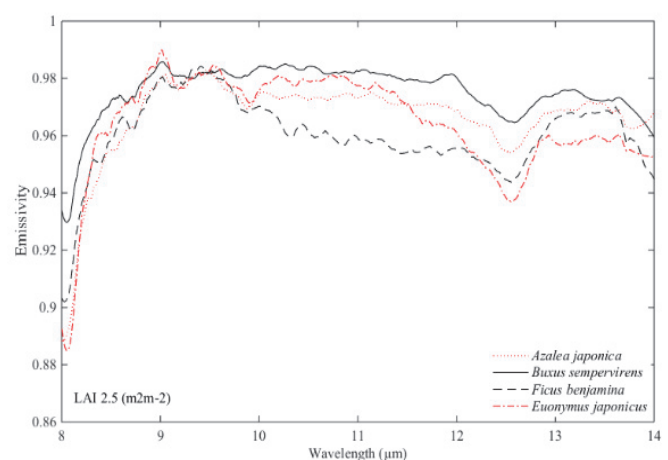

(b)

Figure 2.3: The mean canopy emissivity spectra of different plant species with similar LAI value of (a) 1.5 $\left(\mathrm{m}^{2} \mathrm{~m}^{-2}\right)$, (b) $2.5\left(\mathrm{~m}^{2}\right.$ $\left.\mathrm{m}^{-2}\right)$, and (c) $3.5\left(\mathrm{~m}^{2}\right.$ $\left.\mathrm{m}^{-2}\right)$. Plant species that are not statistically significant from each other are shown in red. 
Table 2.3: Summary statistics from One-way ANOVA with Post-hoc Tukey's HSD test of canopy emissivity spectra for four plant species with LAI value binned around 1.5, 2.5 and $3.5\left(\mathrm{~m}^{2} \mathrm{~m}^{-2}\right)$.Where (1) is Azalea japonica, (2) is Buxus sempervirens, (3) is Ficus benjamina, and (4) is Euonymus japonicus.

\begin{tabular}{|c|c|c|c|c|}
\hline \multirow{2}{*}{ LAI $\left(\mathbf{m}^{2} \mathbf{m}^{-2}\right)$} & \multicolumn{2}{|c|}{ Variable } & \multirow{2}{*}{$\begin{array}{l}\text { Mean Difference } \\
\text { (I-J) }\end{array}$} & \multirow{2}{*}{$p$-value } \\
\hline & (I) & (J) & & \\
\hline \multirow{6}{*}{1.5} & & 2 & $-0.013^{*}$ & $<0.001$ \\
\hline & 1 & 3 & $0.005^{*}$ & 0.008 \\
\hline & & 4 & $-0.005^{*}$ & 0.007 \\
\hline & 2 & 3 & $0.018^{*}$ & $<0.001$ \\
\hline & & 4 & $0.007^{*}$ & $<0.001$ \\
\hline & 3 & 4 & $-0.010^{*}$ & $<0.001$ \\
\hline \multirow{6}{*}{2.5} & & 2 & $-0.010^{*}$ & $<0.001$ \\
\hline & 1 & 3 & $0.004^{*}$ & 0.011 \\
\hline & & 4 & -0.000 & 0.905 \\
\hline & & 3 & $0.014^{*}$ & $<0.001$ \\
\hline & 2 & 4 & $0.009^{*}$ & $<0.001$ \\
\hline & 3 & 4 & $-0.004^{*}$ & 0.007 \\
\hline \multirow{6}{*}{3.5} & & 2 & $-0.006^{*}$ & $<0.001$ \\
\hline & 1 & 3 & 0.001 & 0.212 \\
\hline & & 4 & $-0.002^{*}$ & 0.024 \\
\hline & & 3 & $0.008^{*}$ & $<0.001$ \\
\hline & 2 & 4 & $0.004^{*}$ & 0.003 \\
\hline & 3 & 4 & $-0.004^{*}$ & 0.003 \\
\hline
\end{tabular}

\subsubsection{Principal component analysis}

The PCA analysis revealed that the proportion of the total variance between plant species that was explained by the first principal component $\left(\mathrm{PC}_{1}\right)$ was 97.28\% for species with an LAI around $1.5\left(\mathrm{~m}^{2} \mathrm{~m}^{-2}\right), 93.43 \%$ for species with an LAI value around $2.5\left(\mathrm{~m}^{2} \mathrm{~m}^{-2}\right)$, and $94.07 \%$ for species with an LAI around $3.5\left(\mathrm{~m}^{2} \mathrm{~m}^{-2}\right)$. The second principal component $\left(\mathrm{PC}_{2}\right)$ explained only $1.69 \%$, $4.15 \%$ and $5 \%$ of the variance for LAI values of $1.5,2.5$ and $3.5\left(\mathrm{~m}^{2} \mathrm{~m}^{-2}\right)$ respectively. Therefore, only $\mathrm{PC}_{1}$ was selected for determining the wavebands for which the canopy emissivity spectra of different species with similar LAI values were highly variable. The waveband regions that provided the highest factor loadings were similar for all species with LAI 1.5, 2.5, and $3.5\left(\mathrm{~m}^{2} \mathrm{~m}^{-}\right.$ ${ }^{2}$ ), and were located in $8-10 \mu \mathrm{m}$ part of the electromagnetic spectrum; they included the 8.0-8.3 $\mu \mathrm{m}, 8.8-9.1 \mu \mathrm{m}, 9.4-9.6 \mu \mathrm{m}$, and 9.9-10.1 $\mu \mathrm{m}$ regions. 


\subsection{Discussion}

Earlier studies using TIR hyperspectral data focused mainly on leaf scale measurements and their related properties (e.g. water content, and leaf chemical constituents) (Buitrago et al. 2016; Ribeiro da Luz and Crowley 2010); to our knowledge, to date there have been no studies on the biophysical properties at canopy scale in the TIR domain. We believe our study is the first to use thermal hyperspectral data in order to understand the canopy emissivity spectral response to variation in LAI. Further, it explored whether canopy emissivity spectra of different plant species can be discriminated while their LAI is constant. The analysis of the canopy emissivity spectra of the four plant species revealed discrepancies due to dissimilarities between species in the absolute value of emissivity at different spectral wavebands. The finding demonstrates the potential of TIR hyperspectral data measured at the canopy level for discriminating between various plant species when their LAI is similar.

It is well known that LAI variability affects reflectance signatures in the VNIR portion of the spectrum. Here, we demonstrate that canopy emissivity spectra are also influenced by increasing LAI values in the $8-12 \mu \mathrm{m}$ region. For LAI values greater than $4.0\left(\mathrm{~m}^{2} \mathrm{~m}^{-2}\right)$, the changes in canopy emissivity spectra were less pronounced (Figure 2.2). This suggests that other biophysical (such as leaf angle) and biochemical properties contribute to canopy emissivity spectra; this merits further investigation. In our study, it was observed that the small changes in LAI values (i.e. $<0.5, \mathrm{~m}^{2} \mathrm{~m}^{-2}$ ) are not readily detectable via canopy emissivity spectra. Our results confirmed earlier results obtained by Ullah (2013) that plants have relatively high emissivity and only small and subtle features in the TIR region, and this also applies to measurements made at canopy level.

Plant canopy temperature exerts strong control over respiration and transpiration. LAI is the critical parameter in canopy evaporation (van den Hurk et al. 2003), and transpiration: its variability affects biophysical and biochemical processes through its impact on heat and water fluxes between vegetation and the atmosphere. Moreover, canopy photosynthesis varies according to change in LAI value and the arrangement of the angular distribution of leaves (Peri 2005). In addition, as the LAI of a canopy increases, solar absorption increases because more leaves are present to 
absorb photons. The effects of the changes in leaf solar absorptance are significant in terms of affecting leaf temperature. In other words, changes in LAI values can determine the interception of solar radiation by plants. Therefore, it seems raising the LAI increases the absolute value of emissivity.

Many researchers have investigated the retrieval of LAI from reflectance spectra using optical remote sensing. Our finding is in agreement with previous findings by Darvishzadeh et al. (2008a) who demonstrated that plant species with similar LAI values have different canopy reflectance spectra in the VNIR and SWIR regions. The finding that the canopy emissivity spectra of various plant species with the same LAI value were quite distinct (Figure 2.3) suggests that LAI is probably not the sole factor affecting the canopy emissivity spectra. We know that at leaf level there are factors that affect emissivity (Buitrago et al. 2016; Ullah et al. 2013; Ullah et al. 2014) but probably other canopy structural parameters also contribute to the variation of the canopy emissivity spectra. This might be attributed to the differences in canopy architecture (Pinter Jr et al. 1985), leaves (i.e. size and orientation), canopy gap size (Ribeiro da Luz and Crowley 2010), and concentrations of biochemical factors (Curran et al. 2001) or interactions among these factors. Moreover, the outermost layers of the leaf (i.e. plant cuticle) are claimed to be a principal cause of emissivity spectral features for different plant species in the TIR region (Ribeiro da Luz and Crowley 2007). The external walls of plant epidermal cells are unique for every plant species (Glover 2000). Therefore, the differentiation observed in emissivity spectral for various plant species with similar LAI value might be due to differences in the composition of the external walls of plant epidermal cells. As can be seen from Figure 2.2 canopy emissivity spectra reached saturated at different levels for the different species. A possible explanation is that leaf biophysical variables such as internal leaf structure and leaf orientation as well as pigments are different within the studied species (Baret and Guyot 1991; Yoder and Waring 1994). Additionally, our results are partly in line with the findings of Ullah et al. (2012a), who determined the important wavebands for discriminating between plant species at leaf level. We too found that waveband $9.4 \mu \mathrm{m}$ is one of the most important wavebands, as it yields sufficient information to discriminate between plant species with similar LAI value at canopy level.

Finally, it should be noted that variation in LAI affects the emissivity of the canopy and could potentially affect plant strategies to cope with 
environmental conditions in cases where a plant needs to regulate energy. However, further research is essential to understand the impact of other biophysical and biochemical variables on canopy emissivity spectra in the TIR region.

\subsection{Conclusions}

This study demonstrated the feasibility of using TIR hyperspectral data to explore the influence of LAI on high-resolution emissivity spectra with rising LAI values, the canopy emissivity spectra increased. Further, the results also demonstrated that different plant species had different canopy emissivity spectra even when LAI values were similar. The ability to utilize laboratory spectral measurements to analyse the TIR hyperspectral data at canopy level is also an encouraging result and the first of its kind. General knowledge on the possible relation between vegetation properties and emissivity spectral variation for plant species is still limited. Therefore, efforts should be devoted to exploring the advantages and limitations of TIR hyperspectral data for vegetation studies, especially at the canopy level. This study proved that under controlled laboratory conditions, measurements could also be made at canopy level, and it has shown that hyperspectral TIR remote sensing data are worthwhile analysing in order to study vegetation. 


\section{Chapter 3}

\section{Retrieval of Leaf Area Index in Different Plant Species Using Thermal Hyperspectral Data*}

* This chapter is based on:

Neinavaz, E., Skidmore, A. K., Darvishzadeh, R., \& Groen, T. A. (2016). Retrieval of Leaf Area Index in Different Plant Species using Thermal Hyperspectral Data. ISPRS Journal of Photogrammetry and Remote Sensing, 119, 390-401.

Neinavaz, E., Skidmore, A. K., Darvishzadeh, R., \& Groen, T. A. (2016). Leaf Area Index Retrieved from Thermal Hyperspectral Data. ISPRS-International Archives of the Photogrammetry, Remote Sensing and Spatial Information Sciences, 99-105.

Neinavaz, E., Skidmore, A. K., Darvishzadeh, R., \& Groen, T. A. Retrieval of Leaf Area Index in Different Plant Species using Thermal Hyperspectral Data. XXIII ISPRS Congress, 12-16 July 2016, Prague, Czech Republic. 


\begin{abstract}
Leaf area index (LAI) is an important variable of terrestrial ecosystems because it is strongly correlated with many ecosystem processes (e.g. water balance and evapotranspiration) and directly related to the plant energy balance and gas exchanges. Although LAI has been accurately predicted using visible-near infrared (VNIR, 0.3-1.0 $\mu \mathrm{m}$ ), and short-wave infrared hyperspectral data (SWIR, 1.0-2.5 $\mu \mathrm{m}$ ), LAI estimation using thermal infrared (TIR, 8.0-14.0 $\mu \mathrm{m}$ ) measurements has not yet been addressed. The novel approach of this study is to evaluate the retrieval of LAI using TIR hyperspectral data. The leaf area indices were destructively acquired for four plant species: Azalea japonica, Buxus sempervirens, Euonymus japonicus, and Ficus benjamina. Canopy emissivity spectral measurements were obtained under controlled laboratory conditions using a MIDAC (M4401-F) spectrometer. The LAI retrieval was assessed using a partial least squares regression (PLSR), artificial neural networks (ANN), and narrow band indices calculated from all possible combinations of waveband pairs for three vegetation indices including simple difference, simple ratio, and normalized difference. ANN retrieved LAI more accurately than PLSR and vegetation indices $\left(0.67<\mathrm{R}^{2}<0.95\right.$ versus $\left.11.54<\mathrm{RMSE}_{\mathrm{CV}}<31.23, \%\right)$. The accuracy of LAI retrieval did not differ significantly between the vegetation indices. The results revealed that wavebands from the $8-12 \mu \mathrm{m}$ region contain relevant information for LAI estimation, irrespective of the chosen vegetation index. Moreover, they demonstrated that LAI might be successfully predicted from TIR hyperspectral data, even for higher values of LAI (LAI $\geq 5.5$ ). The study showed the significance of using PLSR and ANN as multivariate methods compared to the univariate technique (e.g. narrow-band vegetation indices) when hyperspectral thermal data is utilized. We thus demonstrated for the first time the potential of hyperspectral thermal data to accurately retrieve LAI.
\end{abstract}




\subsection{Introduction}

Among canopy biophysical variables, leaf area index (LAI, $\mathrm{m}^{2} \mathrm{~m}^{-2}$ ) is of particular interest, as it is a key input for climate and large-scale ecosystem models (Myneni et al. 1997; Wang et al. 2004; Zheng and Moskal 2009). LAI has been successfully modeled in the visible/near-infrared $0.3-1.0 \mu \mathrm{m}$ (VNIR) and Short-wave Infrared 1.0-2.5 $\mu \mathrm{m}$ (SWIR) regions of the electromagnetic spectrum using hyperspectral and multispectral data (Asrar et al. 1984; Zheng and Moskal 2009) and also LIDAR (Riaño et al. 2004) and TLS data (Morsdorf et al. 2006). In the VNIR and SWIR regions, vegetation indices have been related to LAI through linear or exponential regression models (Schlerf et al. 2005). Other studies have confirmed that vegetation indices have a decreasing sensitivity to rising LAI values when optical multispectral and hyperspectral data are used (Baret and Guyot 1991; Broge and Mortensen 2002; Chen 1996; Turner et al. 1999). Darvishzadeh et al. (2009), showed that linear and exponential regression models are similarly accurate at estimating LAI from hyperspectral data.

However, to our knowledge, the relation between vegetation indices and LAI in the thermal infrared 8-14 $\mu \mathrm{m}$ (TIR) region remains unexplored. Thermal infrared data complementary to other remote sensing data and has great potential to elucidate the biophysical or biochemical characteristics of vegetation (Ullah 2013). In particular, the primary absorption features that are associated with certain vegetation components (e.g. water, polysaccharides such as cellulose) and leaf structure parameters (e.g. thickness) are only observable in the mid-wave infrared 3-5 $\mu \mathrm{m}$ (MIR) and TIR regions (Fabre et al. 2011b; Gerber et al. 2011; Ribeiro da Luz 2006). In addition, different plant species show not only distinct reflectance curves in the VNIR and SWIR regions (Schmidt and Skidmore 2003) but also have distinguishable spectral features in the TIR domain (Ribeiro da Luz and Crowley 2010). The TIR spectral profiles have been related to plant species leads us to suspect that TIR may be used to retrieve vegetation parameters such as LAI.

There are a number of challenges when using TIR hyperspectral remote sensing data for vegetation studies. One is the practical difficulty of accurately estimating the emissivity values of plants. In the TIR domain, spectra mainly result from the emissivity (radiative emission) of surfaces, rather than from their reflectance. In vegetation, emissivity characteristics of leaves are subtle, 
and variations in vegetation emissivity spectra are small while plants have relatively high emissivity value in the TIR region (Ribeiro da Luz and Crowley 2007). In addition, only a few instruments measure TIR emissivity at high spectral resolution. A new generation of TIR hyperspectral instruments operates at wavelengths longer than $2.5 \mu \mathrm{m}$ with high spectral resolution (e.g. MIDAC illuminator Fourier Transform Infrared spectrometer: FTIR). These technical developments have created opportunities for examining plant emissivity characteristics and understanding the potential use of the TIR hyperspectral data in vegetation studies, especially at leaf level. TIR hyperspectral data deserves the same level of investigation and commitment to exploring and development, as hyperspectral data in the VNIR and SWIR domains. Thermal infrared data can be expected to complement other remote sensing data.

Few studies have focused on TIR hyperspectral data for vegetation studies at canopy level (Ribeiro da Luz and Crowley 2010; Sepulcre-Cantó et al. 2006), and more work needs to be done to understand TIR remotely measured spectra from vegetation. When Ribeiro da Luz and Crowley (2010) investigated the identification of plant species using canopy spectral features in the TIR region $(8.0-13.5 \mu \mathrm{m})$ from the SEBASS airborne sensor they found that leaves (i.e. size and orientation) and canopy gap sizes influence the identification of plant species from TIR hyperspectral data at the canopy level. Given their preliminary results, as well as those of Ullah et al. (2014), who retrieved leaf water content from TIR hyperspectral data, it seems likely that TIR hyperspectral data provide a valuable opportunity to retrieve LAI.

The concept of vegetation indices was developed in the 1970s (Elvidge and Chen 1995). Earlier studies illustrated the feasibility of using red and near-red infrared bands for vegetation studies (Chen et al. 2005a; Ramoelo et al. 2012). Vegetation indices which are calculated from different combinations of bands are divided into two main categories: orthogonal and ratio indices (Baret and Guyot 1991). The orthogonal indices include indices such as the perpendicular index and difference index (Richardson and Weigand 1977; Tucker 1979) while the ratio-based indices include indices like simple ratio index (Jordan 1969; Pearson and Miller 1972) and normalized difference index (Rouse et al. 1974). Consequently, LAI has been estimated in numerous studies using hyperspectral data in the VNIR and SWIR regions using univariate models such as vegetation indices. This has led to the development 
of new vegetation indices which have further improved LAI retrieval (Asner and Martin 2008; Baret and Guyot 1991; Darvishzadeh et al. 2009; Eriksson et al. 2006; Gao et al. 2000; Haboudane et al. 2004; Koetz et al. 2005). However, when vegetation indices are used, only a limited number of spectral data from the massive spectral content of hyperspectral data are utilized, and therefore it has been suggested (De Jong et al. 2003) to use multivariate methods, as these benefit from the spectral information from several wavebands. These methods include models such as partial least squares regression (PLSR) and artificial neural networks (ANN), which as so-called "full spectrum methods" employ all available spectral data simultaneously (Atzberger et al. 2010) and reduce the effects of multicollinearity as a common problem inherent to hyperspectral dataset (Mirzaie et al. 2014). However, to our knowledge in the TIR, neither the relation between LAI and univariate methods such as vegetation indices nor the relation between LAI and multivariate methods in the TIR region, have been explored. Therefore, the main objective of this study was to investigate the retrieval of LAI in the TIR region, using narrow band indices as well as PLSR and ANN as multivariate methods.

\subsection{Materials and methods}

\subsubsection{Leaf area index measurements}

Four plant species of the different structure were selected: Azalea japonica, an evergreen flowering shrub with ovate leaves; Buxus sempervirens, an evergreen shrub with oval leaves; Euonymus japonicus, an evergreen shrub with oval leaves, with finely serrated margins; and Ficus benjamina, which has glossy, oval leaves with an acuminate tip. Good health and physiological conditions of all sampled species were considered. The plants were obtained from a nursery and were maintained at a temperate of approximately $20^{\circ} \mathrm{C}$. In total, 37 plants were used for sampling. To create variations in LAI and its corresponding canopy emissivity measurements, the leaves from the plants were harvested in several steps during which the LAI was regularly reduced by clipping the foliage. The surface areas of harvested leaves were measured at each harvest, using a LI-3000C portable leaf area meter (LICOR, NE, USA) with an accuracy of $1 \mathrm{~m} \mathrm{~m}^{2}$. The instrument was regularly calibrated. As LAI measures the one-sided leaf area $\left(\mathrm{m}^{2}\right)$ per unit of horizontal surface area $\left(\mathrm{m}^{2}\right)$ 
(Watson 1947), here the measured surface areas of the leaves $\left(\mathrm{m}^{2}\right)$ were divided by the corresponding ground area of the canopy $\left(\mathrm{m}^{2}\right)$ to calculate the LAI $\left(\mathrm{m}^{2} \mathrm{~m}^{-2}\right)$. The dataset included 37 plant specimens, which yielded 144 LAI estimates from the destructive sampling.

\subsubsection{Canopy thermal infrared radiance measurement}

The radiance spectral measurements were made using a portable MIDAC FTIR spectrometer (Model M4401-F; MIDAC Corporation, CA, USA). The MIDAC configuration enables radiance spectra within the spectral range of $2.5-20 \mu \mathrm{m}$ to be measured at an adjustable spectral resolution of $32-0.5 \mathrm{~cm}^{-1}$. The MIDAC has a liquid nitrogen- cooled Mercury-Cadmium-Telluride detector and customized foreoptics that consist of a flat folding mirror on a rotational axis, which allows two blackbodies (one hot, one cold) to be measured for calibrating each sample. The MIDAC's folding mirror was kept at nadir position above the samples. The MIDAC's field of view (using a cutoff of $5 \%$ of the maximum responsivity) has a starting diameter of $53 \mathrm{~mm}$ at the folding mirror and spreads with about $18 \mathrm{mrad}$ (Eisele et al. 2015). The amount of thermal emission varies according to the distance between the sample and the sensor (Ribeiro da Luz and Crowley 2007) and reducing atmospheric attenuation (Korb et al. 1996) measurements were made with a fixed vertical distance between sensor and sample. To create optimal measurement conditions and reduce any possible source of errors due to the changes in atmospheric conditions or temperature, the measurements were made under controlled laboratory conditions in which the walls, ceiling, and floor were coated with a black material (Avis Aqua Blackboard Black) and black plastic of known emissivity. Traditionally when the emissivity of geological samples is measured, they are heated to a temperature above ambient condition to create a thermal contrast (Ribeiro da Luz and Crowley 2007; Salisbury 1998). However, such a treatment stresses plant samples, so instead, we reduced the lab temperature to $10^{\circ} \mathrm{C}$ in order to generate a suitable thermal contrast with the plants, which were at a higher room temperature. In addition, plants were kept outside the laboratory at an ambient room temperature of $20^{\circ} \mathrm{C}$, and each one was briefly introduced to the cool room in order to make the thermal measurements. In this experiment, the background soil was covered with black plastic of known emissivity to minimize possible effects of soil. The emissivity spectra of the black plastic were measured using 
a BRUKER Vertex 70 laboratory FTIR spectrometer. The black plastic had a very low emissivity so could be assumed to cause minimal interference with the radiance measurement of samples. The radiance spectra of the plant canopies were measured between wavelengths of 2.5-20 $\mu \mathrm{m}$ at a resolution of $2 \mathrm{~cm}^{-1}$. The average of 32 scans was calculated for each LAI sample value. The emissivity spectra of plant canopies at each LAI value were obtained using a series of FTIR measurements performed in the following order: radiance measurements of the hot blackbody, radiance measurement of the cold blackbody, radiance measurement of the sample (i.e. the plant canopy with specific LAI value), and finally, radiance measurements of a highly diffuse reflecting gold plate (Infragold). For instrument radiance calibration, two blackbodies (one hot, one cold) were used. Their temperature was regularly checked between measurements of each sample. The cold blackbody temperature was set just below the ambient temperature, at $5^{\circ} \mathrm{C}$ (Korb et al. 1996). The hot blackbody temperature was set above the sample temperature (Hori et al. 2006; Salvaggio and Miller 2001), at $30^{\circ} \mathrm{C}$. Details of the radiometric calibration applied with the measurements of these blackbodies' radiances can be found in (Hook and Kahle 1996).

A diffuse reflecting gold plate with an emissivity of $\sim 0.04$ was used to collect down-welling radiance (DWR) in order to determine the quantitative influence of the laboratory background self-emission (Eisele et al. 2015). The infragold plate was placed directly under the MIDAC sensor at the same distance as the sample. The temperatures of the sample, infragold plate and lab were frequently monitored before and after each measurement, using thermistors (FLUKE 51 II Thermometer and Precision IR Thermometer) to detect any possible changes in temperature that could affect the measurements. Changes in temperature can disturb the thermal contrast, which then causes the emissivity value to be over- or underestimated. The measurement series were taken within five minutes to minimize the possible drift of the instrument, physiological changes in the plants, and fluctuations in laboratory temperature (Hori et al. 2006). After each set of measurements, the canopy was rotated $90^{\circ}$ clockwise. The final corresponding canopy emissivity spectra of each sample (for a specific LAI value), was then calculated from the average of four sets of measurements (covering $360^{\circ}$ ) (see Figure 3.1). 


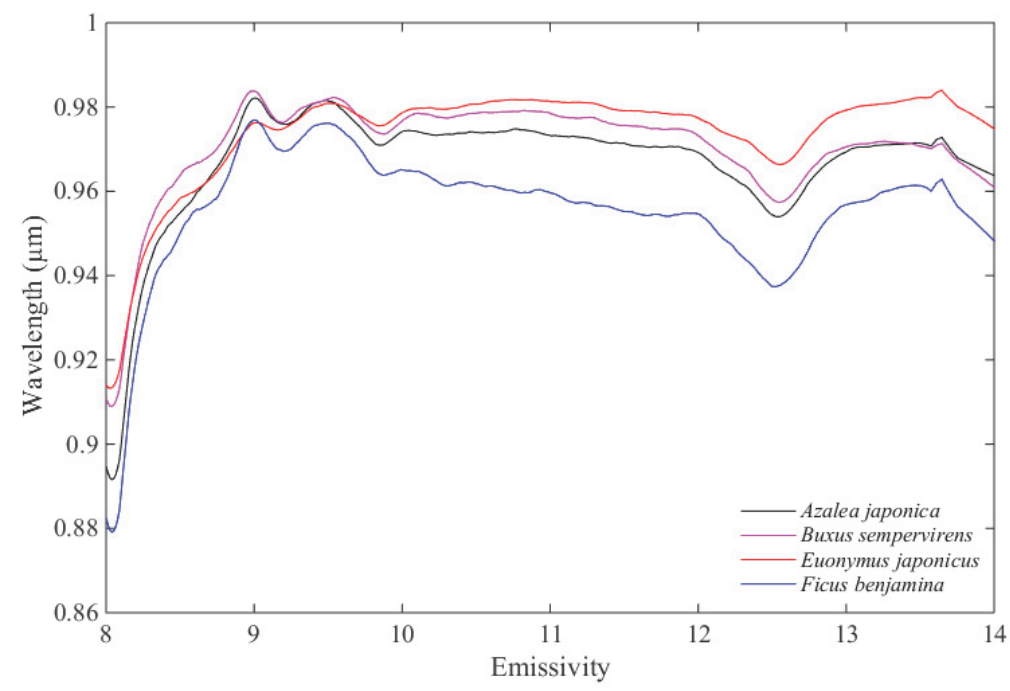

Figure 3.1: The canopy emissivity spectra of four plant species in a thermal infrared region corresponding between $8 \mu \mathrm{m}$ and $14 \mu \mathrm{m}$.

\subsubsection{Data processing and analysis}

Spectral emissivity of the plants was calculated from their absolute radiance using the following equation (Korb et al. 1996),

$\varepsilon_{\text {sam }}(\lambda)=\frac{\mathrm{L}_{\text {sam }}(\lambda)-\mathrm{L}_{\mathrm{DWR}}(\lambda)}{\mathrm{B}\left(\lambda, \mathrm{T}_{\mathrm{sam}}\right)-\mathrm{L}_{\mathrm{DWR}}(\lambda)}$

where $\varepsilon_{\text {sam }}(\lambda)$ denotes the directional emissivity of the sample at the wavelength $\lambda, L_{\text {sam }}(\lambda)$ is spectral radiance from the target, $T_{\text {sam }}$ is the true physical temperature of the sample, $B\left(\lambda, \mathcal{E}_{\text {sam }}\right)$ is the Planck function at the wavelength $\lambda$ and sample temperature, and $L_{D W R}(\lambda)$ is total spectral DWR from the hemisphere above the sample Direct temperature measurement $\left(T_{\text {sam }}\right)$ must accurately represent the emission temperature. Therefore, after measuring canopy temperature before and after each measurement, the blackbody fit method was used to calculate the sample emissivity value. Details about the blackbody fit method can be found in Kahle and Alley (1992) and Salvaggio and Miller (2001). The emissivity of 279 wavebands between $8 \mu \mathrm{m}$ and $14 \mu \mathrm{m}$ was selected for analysis; measurements outside this range had a very low signal strength. A Savitzky-Golay filter with a frame size of 15 data points and second-degree polynomial was used to reduce the 
noise of the canopy emissivity spectra (Savitzky and Golay 1964). Data were analyzed and processed using MATLAB R2013b (Mathwork, Inc.).

\subsubsection{Estimation of leaf area index}

\subsubsection{Narrow band indices}

Vegetation indices, those used most frequently to derive LAI from optical remote sensing data are a simple difference, simple ratio, and the normalized difference (Huete et al. 2002). Therefore, in this study, these widely used indices were calculated from all the possible paired waveband combinations, using canopy emissivity spectra between 8 and $14 \mu \mathrm{m}$. To evaluate the strength of the relation between proposed indices and LAI, the coefficient of determination $\left(\mathrm{R}^{2}\right)$ of all possible two-waveband combinations of vegetation indices (i.e. SD, SR, and ND) and LAI were used. From all these combinations, we selected the thermal narrow band indices that performed best at estimating LAI, as having the maximum $\mathrm{R}^{2}$ with LAI (Mutanga and Skidmore 2004). In addition, the first twenty combination wavebands that have highest $\mathrm{R}^{2}$ were identified and the range of combination was provided for all species. In our study, a linear regression model was used to model the relationship between narrow band indices as the predictor variable and LAI. The naming conventions, acronyms, and calculations for the indices are listed in Table 3.1.

Table 3.1: Spectral indices used in this paper.

\begin{tabular}{cccc}
\hline No. & Name & Acronym TIR & Equation \\
\hline 1 & Simple Ratio & SR & $* \varepsilon_{\lambda 1} / \varepsilon_{\lambda 2}$ \\
2 & Normalized Difference & ND & $\frac{\varepsilon_{\lambda 1}-\varepsilon_{\lambda 2}}{\varepsilon_{\lambda 1}+\varepsilon_{\lambda 2}}$ \\
3 & Simple Difference & SD & $\varepsilon_{\lambda 1}-\varepsilon_{\lambda 2}$ \\
\hline Where $\varepsilon_{\lambda 1}$ and $\varepsilon_{\lambda 2}$ are the canopy emissivity spectra at two different wavebands.
\end{tabular}

\subsubsection{Partial least squares regression}

Hyperspectral data has high spectral dimensionality, and there is a high degree of collinearity in adjacent wavebands. PLSR is a popular multivariate statistical technique for transforming the wavebands to new orthogonal factors or components (Abdi 2003; Helland 1988). PLSR has been broadly applied in remote sensing vegetation analysis (Cho et al. 2007; Clevers and 
Kooistra 2006; Kooistra et al. 2004). Here, the PLSR analysis was used to determine the relative contribution of LAI to the 279 wavebands canopy emissivity spectra for all the sampled species. The canopy emissivity spectra as independent variables were mean-centered before performing the PLSR analysis. To avoid over-fitting and prevent collinearity, an optimum number of factors was determined, using a cross-validation procedure. The criterion for adding an extra factor to the model was that it decreased cross-validated root mean squared error $\left(\mathrm{RMSE}_{\mathrm{CV}}\right)$ by $>2 \%$ (Geladi and Kowalski 1986). The accuracy of the models was evaluated using RMSECv since the predicted samples were not the same as the samples utilized to build the models. In this regard, lower values of RMSEcv indicate a better fit. All PLSR analyses were carried out using the TOMCAT toolbox 1.01 within MATLAB (Daszykowski et al. 2007). Since the calibration equation coefficients ( $\beta$ coefficients) represent the contribution of each waveband to the model, they were utilized to determine the importance of wavebands in the PLSR model. The thresholds for $\beta$ coefficients were specified according to their $\beta$ coefficients standard deviation (Haaland and Thomas 1988). Wavebands with a $\beta$ coefficient above this threshold were considered to be contributing significantly. The sign of the $\beta$ coefficients (i.e. plus or minus) represents the direction of the relationship between emissivity spectra and LAI.

\subsubsection{Artificial neural networks}

The use of ANN has been found to be an effective alternative to more traditional statistical techniques (Danson et al. 2003; Paliwal and Kumar 2009). The multi-layer perceptron is a broadly utilized neural network in remote sensing. In common with most ANN, it consists of various layers: inputs, hidden layers, and outputs. In this study, canopy emissivity spectra in 279 wavebands between $8 \mu \mathrm{m}$ and $14 \mu \mathrm{m}$ domains were used for each species separately as inputs. For network training, the Levenberg-Marquardt (LMANN) algorithm was utilized, as it is one of the common training algorithms in backpropagation networks to develop models for LAI estimation. There are no rules for determining the optimal number of hidden layers.

However, increasing the number of hidden layers enables the network to tackle more complex problems (Atkinson and Tatnall 1997). As the prediction

performance of an ANN depends on the number of neurons in the hidden 
layer, the best ANN size was identified by testing different numbers. To avoid over-fitting, the early stopping technique was applied. In this technique, training stops as soon as performance on a validation data set begins to worsen (Nowlan and Hinton 1992). Linear regression analyses were performed between the predicted LAI (i.e. the network outputs) and the measured LAI, to identify the best ANN model. To reduce undesired effects from the random initialization of the optimization routine, the cross-validation procedure was repeated 1000 times, and results were averaged. The reliability of the ANN in estimating LAI was evaluated using cross-validated coefficient of determination $\left(\mathrm{R}^{2} \mathrm{CV}\right)$ and $\mathrm{RMSE}_{\mathrm{CV}}$. All analyses were executed using the MATLAB neural network toolbox.

\subsubsection{Validation}

A cross-validation procedure also known as the "leave one out method" (Duda and Hart 1973), was used to validate the regression models. Cross-validation is a method for selecting a model according to its predictive ability (Shao 1993). In cross validation, each sample is estimated using a model fitted on the remaining samples. In other words, each data point is successively "left out" from the data and used as the validation set. The estimated LAI was assessed using the cross-validated coefficient of determination $\left(\mathrm{R}^{2} \mathrm{CV}\right)$, and RMSE $_{C V}$ in order to identify the best performing narrow band index, PLSR, and ANN models. RMSECV has the same unit as the dependent variable; however, here the unit of $\mathrm{RMSE}_{\mathrm{CV}}$ is represented in percentage (\%) in applied methods.

\subsection{Results}

\subsubsection{Narrow band indices and leaf area index}

The experimental setup ensured a wide range of variation in LAI. In total, 144 LAI values varying between $0.60\left(\mathrm{~m}^{2} \mathrm{~m}^{-2}\right)$ and a maximum of $9.80\left(\mathrm{~m}^{2} \mathrm{~m}^{-2}\right)$ were created (Table 3.2). The plant species with the lowest LAI was Azalea japonica (3.35); the highest LAI was found in Buxus sempervirens (9.80). Due to the variation in LAI values, the measured emissivity at the canopy level exhibited a large variability. 
Table 3.2: Summary statistics of the leaf area index (LAI) measurements and emissivity values for four plant species $(n=144)$.

\begin{tabular}{ccccccccc}
\hline \multirow{2}{*}{ Species name } & \multicolumn{3}{c}{ LAI $\left(\mathbf{m}^{\mathbf{2}} \mathbf{~ m}^{-\mathbf{2}}\right)$} & \multicolumn{3}{c}{ Emissivity value } & \multicolumn{2}{c}{ Sample } \\
\cline { 2 - 9 } & Mean & Max & Min & Mean & Max & Min & No. & size \\
\hline Azalea japonica & 1.57 & 3.35 & 0.60 & 0.96 & 0.99 & 0.82 & 10 & 30 \\
Buxus sempervirens & 4.54 & 9.80 & 1.17 & 0.96 & 0.99 & 0.81 & 10 & 40 \\
Euonymus japonicus & 3.28 & 7.43 & 1.25 & 0.97 & 0.99 & 0.84 & 11 & 44 \\
Ficus benjamina & 3.60 & 8.36 & 1.04 & 0.95 & 0.99 & 0.81 & 6 & 30 \\
\hline Total & 3.25 & 9.80 & 0.60 & 0.95 & 0.99 & 0.81 & 37 & 144 \\
\hline
\end{tabular}

Before analysis of vegetation indices, the correlation between LAI and canopy emissivity spectra of individual wavebands was quantified using Pearson's $r$. There was a positive correlation between LAI and emissivity in the TIR domain. Some correlations were statistically significant for wavebands across the $8-14 \mu \mathrm{m}$ range (Figure 3.2). The maximum correlation varied among species and was observed almost in different regions of the TIR portion of the electromagnetic spectrum. For instance, in Azalea japonica, the maximum correlation $(\mathrm{r}=0.46, p$-value $=0.009)$ was observed at the $10.0 \mu \mathrm{m}$ wavelength, while for Euonymus japonicus it was also $\mathrm{r}=0.46$, but $p$-value $<$ 0.001 and observed at $9.3 \mu \mathrm{m}$, and for Buxus sempervirens, it was $\mathrm{r}=0.36, p$ value $=0.04$, observed at $9.0 \mu \mathrm{m}$. By contrast, for Ficus benjamina the maximum value of correlation was $(\mathrm{r}=0.48, p$-value $=0.006)$ at $12.0 \mu \mathrm{m}$.\#.

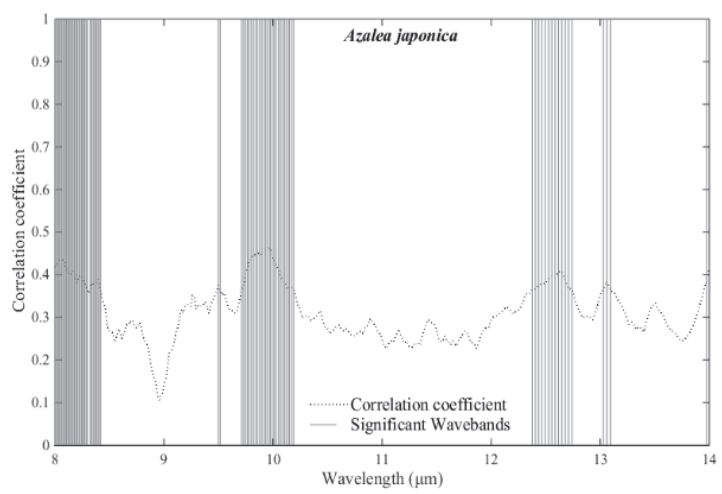

(a) 


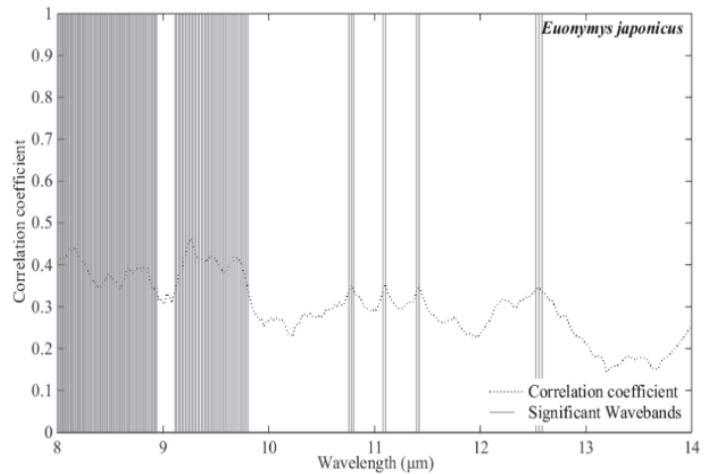

(b)

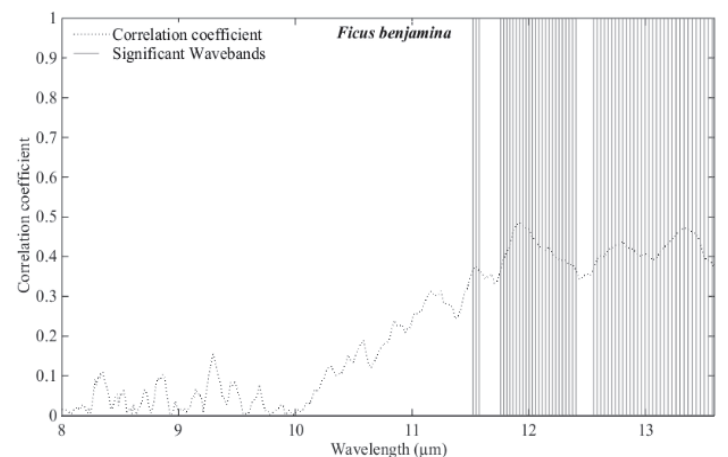

(c)

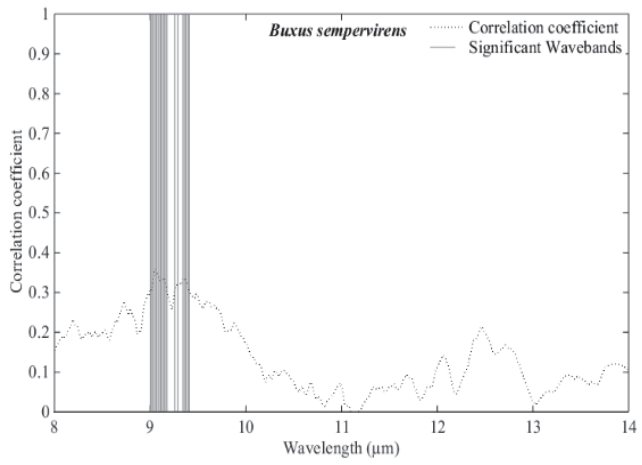

(d)

Figure 3.2: The relationship between canopy emissivity spectra at individual wavelengths and leaf area index in Azalea japonica (a), Euonymus japonicus (b), Ficus benjamina (c), and Buxus sempervirens (d). The gray lines represent the wavebands at which the canopy emissivity spectra correlated statistically significantly with leaf area index.

The $\mathrm{R}^{2}$ between these narrow band indices and LAI were computed. Figure 3.3 depicts these results for all species in a 2-D correlation plot where the 
meeting point of each pair of wavebands corresponds to the $\mathrm{R}^{2}$ value of LAI and the indices calculated from the emissivity values in those two wavebands. Table 3.3 details the highest coefficient of determination between the narrowband thermal indices and the LAI, for each species, as well as the position of best band combinations. The Table 3.3 shows that the highest $\mathrm{R}^{2}$ value obtained between LAI and narrow band indices among species was 0.65 in Ficus benjamina and the lowest was 0.44 in Buxus sempervirens. The results showed that the highest $\mathrm{R}^{2}$ with LAI across species was obtained using the $10-12 \mu \mathrm{m}$ range in combination with the bands in the $8-11 \mu \mathrm{m}$ range. When emissivity measurements of all species were pooled, the $\mathrm{R}^{2}$ between LAI and indices dropped. Accordingly, the LAI estimated from the pooled data was much less accurate than the LAI values estimated for each individual species (SD: $\mathrm{R}^{2}=0.22$ ). For the best performing narrow-band indices, cross-validated $\mathrm{R}^{2} \mathrm{CV}$ and $\mathrm{RMSE} \mathrm{CV}_{\mathrm{C}}$ were computed from linear regression models (Table 3.3). Overall, LAI for all species was predicted with reasonable accuracy. A comparison of $\mathrm{R}^{2} \mathrm{CV}$ and $\mathrm{RMSECV}_{\mathrm{CV}}$ values of the best performing thermal narrow band indices among investigated indices (i.e. SD, SR, and ND) for each species revealed no significant differences among the vegetation indices. The relationship between measured and estimated LAI in all species is presented in Figure 3.4. The scatter plots in Figure 3.4, demonstrated that there was a linear relationship between estimated and measured LAI when the best waveband combinations were used for the three indices. 
Table 3.3: The coefficients of determination $\left(\mathrm{R}^{2}\right)$ of the best performing narrow-band indices and LAI, the wavelength positions and the cross-validated $\mathrm{R}^{2} \mathrm{CV}$ and $\mathrm{RMSE} \mathrm{CV}_{\mathrm{V}}$ for LAI estimated with a linear regression model.

\begin{tabular}{|c|c|c|c|c|c|c|c|c|}
\hline \multirow{2}{*}{$\begin{array}{l}\text { Species } \\
\text { name }\end{array}$} & \multirow[t]{2}{*}{ Index } & \multirow[t]{2}{*}{$\mathbf{R}^{2}$} & \multicolumn{2}{|c|}{$\begin{array}{l}\text { Optimal range } \\
\text { of combination }\end{array}$} & \multicolumn{2}{|c|}{$\begin{array}{c}\text { Most } \\
\text { Sensitive } \\
\text { wavebands }\end{array}$} & \multicolumn{2}{|c|}{ Validation } \\
\hline & & & $\begin{array}{c}\lambda_{1} \\
(\mu \mathrm{m})\end{array}$ & $\begin{array}{c}\lambda_{2} \\
(\mu \mathrm{m})\end{array}$ & $\begin{array}{c}\lambda_{1} \\
(\mu \mathrm{m})\end{array}$ & $\begin{array}{c}\lambda_{2} \\
(\mu \mathrm{m})\end{array}$ & $\mathbf{R}^{2} \mathrm{CV}$ & $\begin{array}{c}\text { RMSE }_{\text {CV }} \\
(\%) \\
\end{array}$ \\
\hline \multirow{3}{*}{$\begin{array}{c}\text { Ficus } \\
\text { benjamina }\end{array}$} & SD & 0.65 & \multirow{3}{*}{$\begin{array}{l}(11.86- \\
11.92)\end{array}$} & \multirow{3}{*}{$\begin{array}{l}(10.62- \\
10.80)\end{array}$} & \multirow{3}{*}{11.92} & \multirow{3}{*}{10.64} & 0.60 & 31.95 \\
\hline & SR & 0.65 & & & & & 0.60 & 31.90 \\
\hline & ND & 0.65 & & & & & 0.60 & 31.87 \\
\hline \multirow{3}{*}{$\begin{array}{c}\text { Azalea } \\
\text { japonica }\end{array}$} & SD & 0.48 & \multirow{3}{*}{$\begin{array}{l}(10.22- \\
10.35)\end{array}$} & \multirow{3}{*}{$\begin{array}{l}(9.95- \\
9.99)\end{array}$} & \multirow{3}{*}{10.28} & \multirow{3}{*}{9.97} & 0.38 & 27.71 \\
\hline & SR & 0.48 & & & & & 0.38 & 27.71 \\
\hline & ND & 0.48 & & & & & 0.38 & 27.71 \\
\hline \multirow{3}{*}{$\begin{array}{l}\text { Euonymus } \\
\text { japonicus }\end{array}$} & SD & 0.64 & \multirow{3}{*}{$\begin{array}{l}(9.83- \\
9.99)\end{array}$} & \multirow{3}{*}{$\begin{array}{l}(9.24- \\
9.27)\end{array}$} & \multirow{3}{*}{9.91} & \multirow{3}{*}{9.25} & 0.57 & 36.87 \\
\hline & SR & 0.64 & & & & & 0.57 & 36.77 \\
\hline & ND & 0.64 & & & & & 0.57 & 36.80 \\
\hline \multirow{3}{*}{$\begin{array}{c}\text { Buxus } \\
\text { sempervirens }\end{array}$} & SD & 0.44 & \multirow{3}{*}{$\begin{array}{l}(9.04- \\
9.09)\end{array}$} & \multirow{3}{*}{$\begin{array}{l}(8.89- \\
8.94)\end{array}$} & \multirow{3}{*}{9.06} & \multirow{3}{*}{8.90} & 0.34 & 41.00 \\
\hline & SR & 0.44 & & & & & 0.34 & 40.98 \\
\hline & ND & 0.44 & & & & & 0.34 & 40.98 \\
\hline \multirow{3}{*}{ Pooled data } & SD & 0.22 & \multirow{3}{*}{$\begin{array}{l}(11.81- \\
11.92)\end{array}$} & \multirow{3}{*}{$\begin{array}{l}(8.50- \\
8.52)\end{array}$} & \multirow{3}{*}{11.86} & \multirow{3}{*}{8.52} & 0.19 & 55.33 \\
\hline & SR & 0.22 & & & & & 0.18 & 55.36 \\
\hline & ND & 0.22 & & & & & 0.19 & 55.29 \\
\hline
\end{tabular}

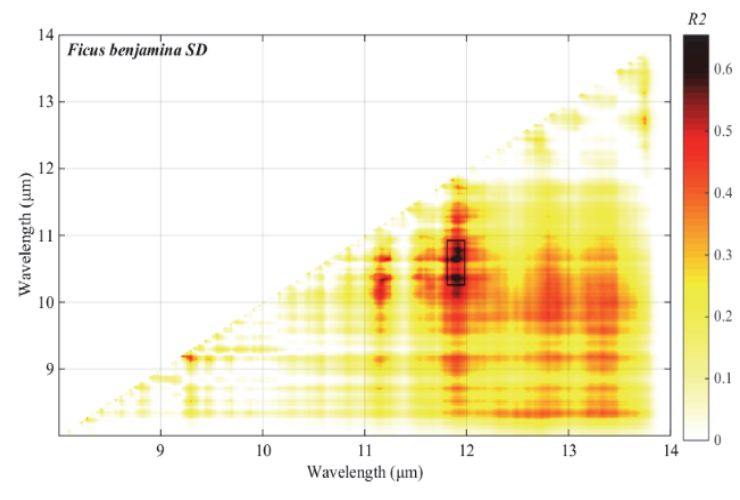

(a) 


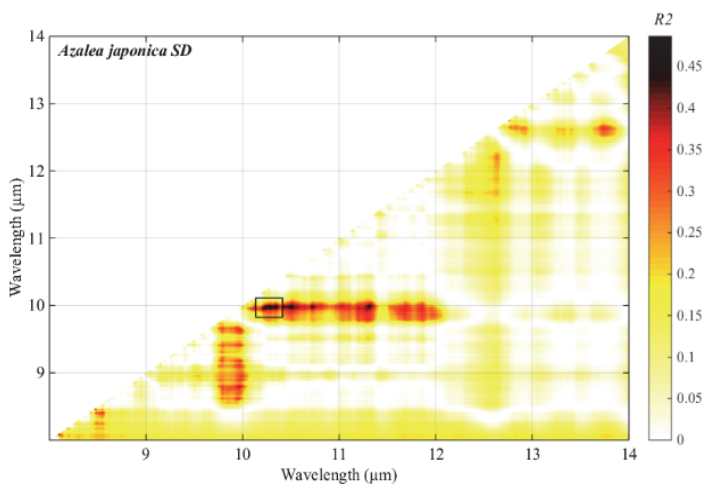

(b)
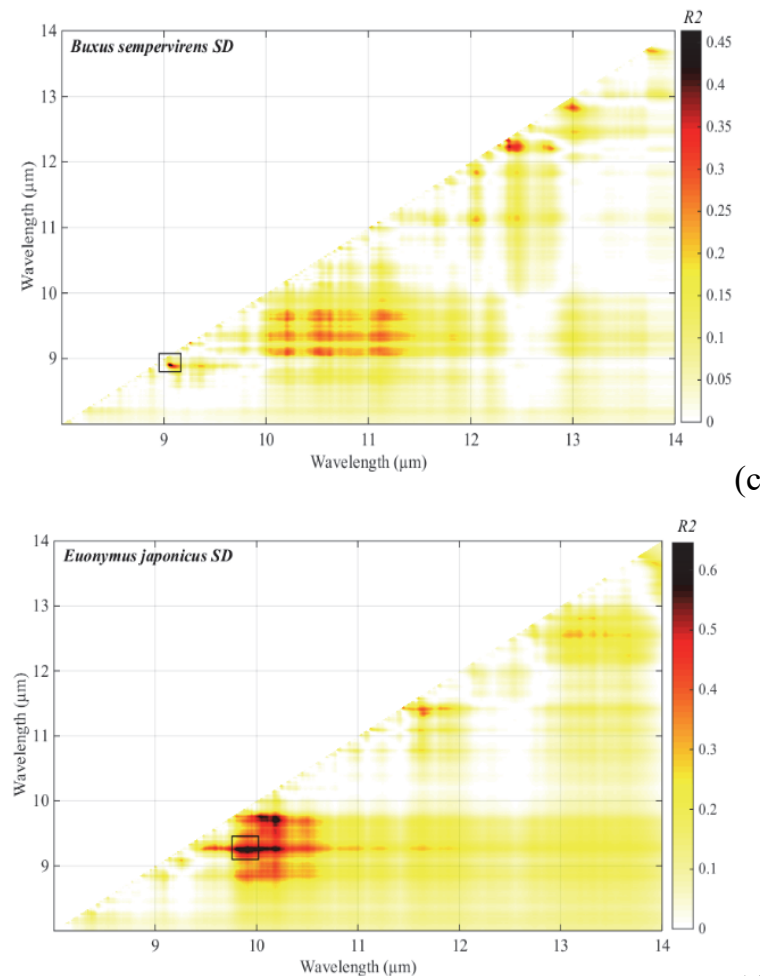

(d) 


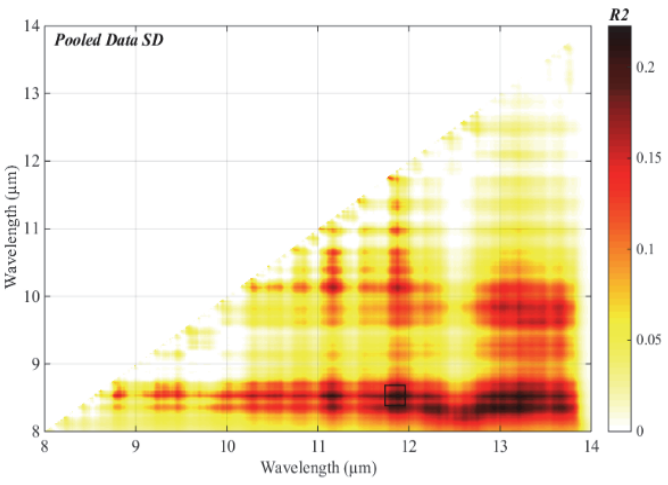

(e)

Figure 3.3: $2-\mathrm{D}$ correlation plots representing the coefficient of determination $\left(\mathrm{R}^{2}\right)$ between leaf area index and simple difference index for Ficus benjamina (a), Azalea japonica (b), Buxus sempervirens (c), Euonymus japonicus (d), and pooled data (e) calculated from all possible two-waveband combinations between $8 \mu \mathrm{m}$ and $14 \mu \mathrm{m}$.

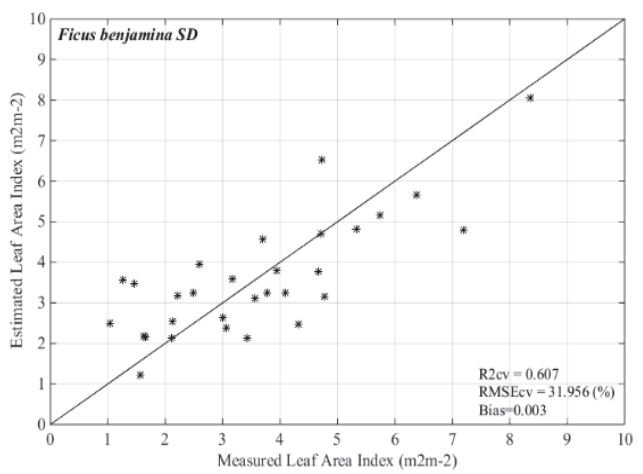

(a)

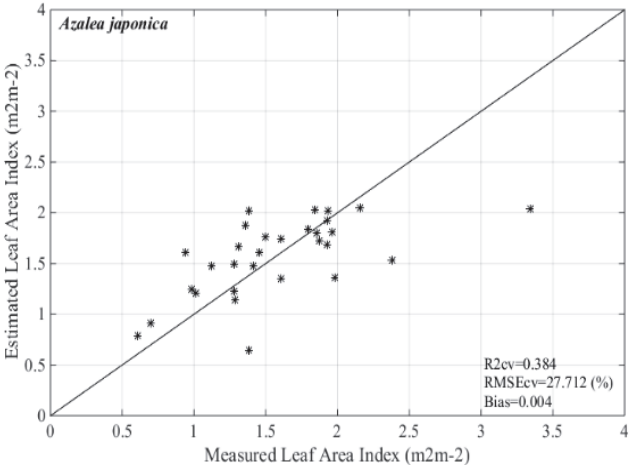

(b) 


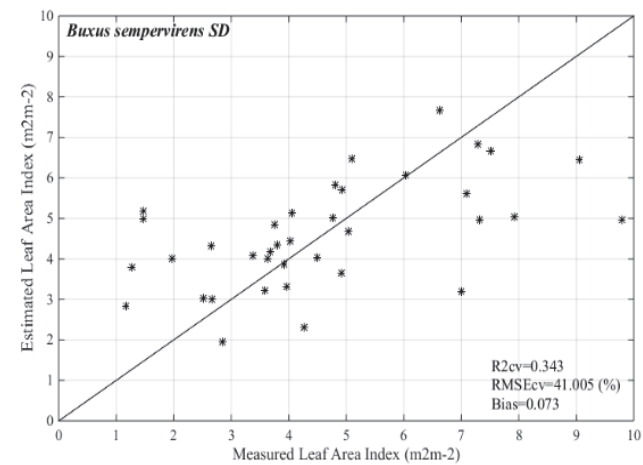

(c)

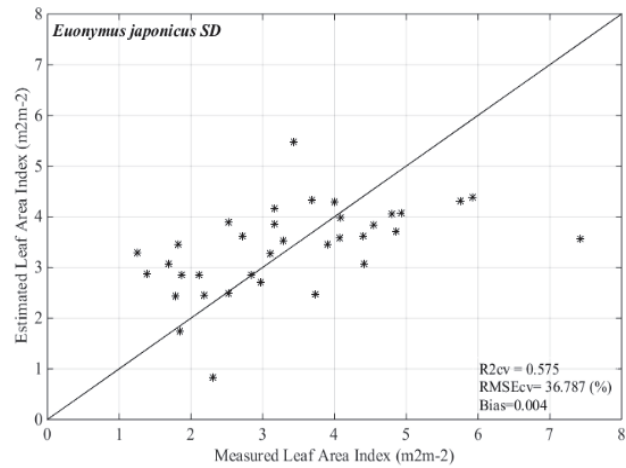

(d)

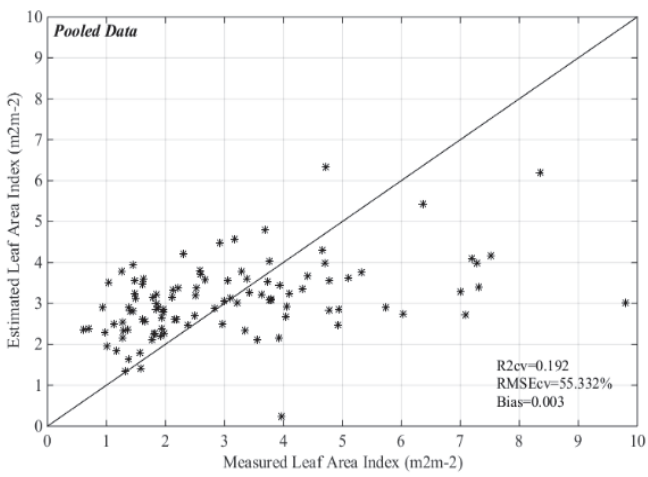

(e)

Figure 3.4: Scatterplot of measured versus predicted leaf area index for the best narrow band indices calculated from simple difference for Ficus benjamina (a), Azalea japonica (b), Buxus sempervirens (c), Euonymus japonicus (d), and pooled data (e). The optimum wavebands are those reported in Table 3.3.

\subsubsection{Partial least squares regression and leaf area index}

The numbers of factors modeled using PLSR were not equal: they ranged from three to five per species (Table 3.4). The PLSR regression using TIR 
spectra yielded the lowest $\mathrm{R}^{2}$ in Azalea japonica and highest $\mathrm{R}^{2}$ in Euonymus japonicus. The important wavebands for each species are tabulated in Table 3.4. Important wavebands in the PLSR model were almost the same as the most sensitive wavebands for vegetation indices and were located in the same part of the spectrum. For all species, the PLSR models in the TIR region yielded better estimates of LAI than the narrow band indices (i.e. resulted in a higher $\mathrm{R}^{2}$ and lower relative $\mathrm{RMSE}_{\mathrm{CV}}$ ). For instance, the significant improvement was noticed when retrieving LAI for Euonymus japonicus, where $\mathrm{R}^{2}$ increased from 0.64 to 0.82 and $\mathrm{RMSE}_{\mathrm{CV}}$ decreased from $36.87 \%$ to $34.06 \%$. Cross-validation results using PLSR for the estimation of LAI for all species are presented in Figure 3.5. The LAI estimated from the pooled data using PLSR was much less accurate than the LAI values estimated for individual species.

Table 3.4: Performance of partial least squares regression (PLSR), and the important wavebands selected by PLSR model for estimating leaf area index.

\begin{tabular}{ccccccc}
\hline Species & $\begin{array}{c}\text { No. of } \\
\text { Factors }\end{array}$ & $\mathbf{R}^{\mathbf{2}}$ & $\begin{array}{c}\text { RMS } \\
\left(\mathbf{m}^{\mathbf{2}} \mathbf{~ m}^{-2}\right)\end{array}$ & $\begin{array}{c}\text { RMSE } \\
(\mathbf{\%})\end{array}$ & $\begin{array}{c}\text { SD of } \boldsymbol{\beta} \\
\mathbf{C o e f}\end{array}$ & $\begin{array}{c}\text { Important } \\
\text { wavebands }(\boldsymbol{\mu m})\end{array}$ \\
\hline Ficus benjamina & 3 & 0.68 & 0.98 & 31.26 & 3.90 & $\begin{array}{c}(11.86-11.94), \\
8.8,(13.75-14.0)\end{array}$ \\
\hline Azalea japonica & 3 & 0.54 & 0.36 & 19.32 & 1.70 & $\begin{array}{c}(9.7-10.02), 8.4 \\
\& 8.5\end{array}$ \\
\hline Euonymus japonicus & 5 & 0.82 & 0.63 & 34.06 & 8.02 & $9.2,(9.9-10.2)$ \\
\hline Buxus sempervirens & 5 & 0.64 & 1.30 & 27.403 & 12.74 & $\begin{array}{c}8.1,9.0,9.1, \\
12.0,12.1,12.3, \\
12.4,13.5 \& 13.6\end{array}$ \\
\hline Pooled data & 4 & 0.22 & 1.65 & 56.75 & & \\
\hline
\end{tabular}




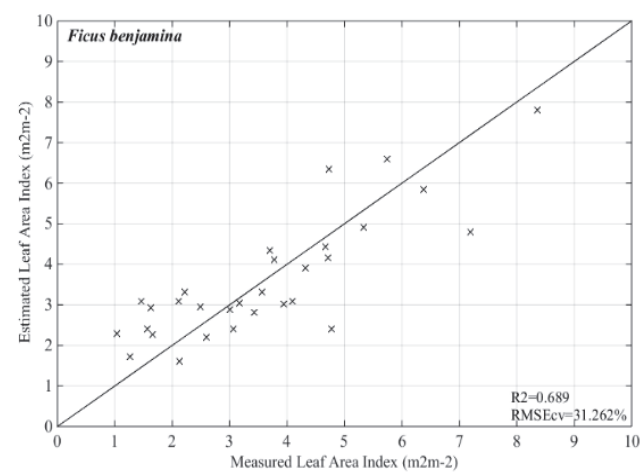

(a)
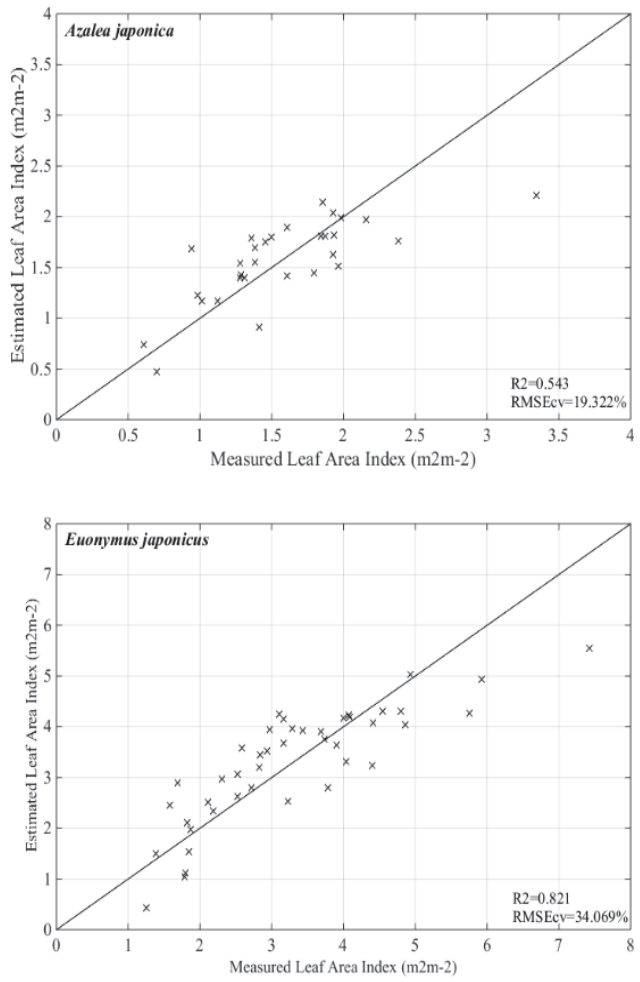

(c) 


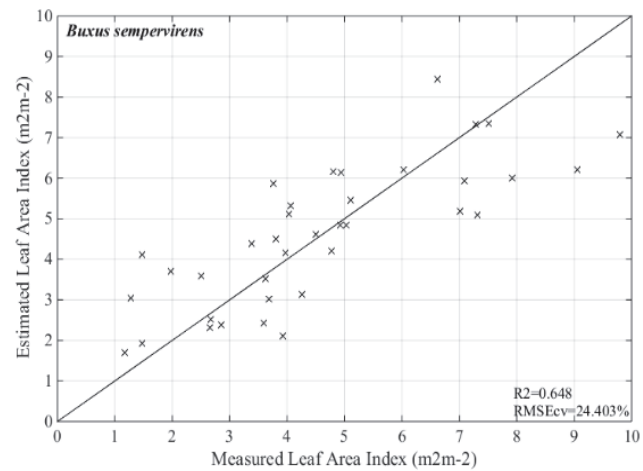

(d)

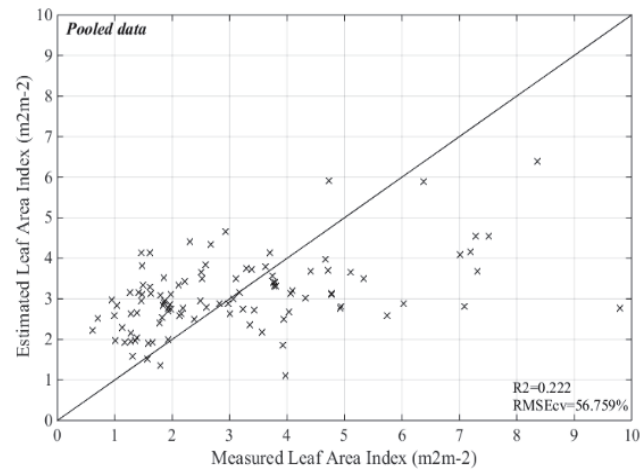

(e)

Figure 3.5: Scatterplot of measured versus estimated leaf area index using entire emissivity spectra in partial least squares regression model from 8-14 $\mu \mathrm{m}$ : Ficus benjamina (a), Azalea japonica (b), Euonymus japonicus (c), Buxus sempervirens (d), and Pooled data (e).

\subsubsection{Artificial neural networks and leaf area index}

A comparative analysis of ANN resulting from the LMANN training algorithm is shown in Table 3.5, which demonstrates the prediction accuracy of the optimum ANN structure for each species. The most accurate prediction of LAI was obtained for Ficus benjamina $\left(\mathrm{R}^{2} \mathrm{CV}=0.95, \mathrm{RMSE}_{\mathrm{CV}}=11.54, \%\right)$. The highest predicted accuracy using ANN was obtained using three neurons per hidden layer. The relationship between estimated and measured LAI using ANN models are shown in Figure 3.6. As can be seen, the accuracy estimation was slightly increased in comparison with PLSR methods. 
Table 3.5: Cross-validated results ( $R^{2}$ and $\left.R M S E\right)$ of the estimated leaf area index obtained using neural networks with different inputs (e.g. four plant species), and network structures. $R^{2} \mathrm{CV}$ and $R M \mathrm{SE}_{\mathrm{CV}}$ represent average results of 1000 random initializations.

\begin{tabular}{ccccc}
\hline Species & Input & $\begin{array}{c}\text { Optimal No. of } \\
\text { neurons }\end{array}$ & $\mathbf{R}^{\mathbf{2}} \mathbf{C V}$ & $\begin{array}{c}\text { RMSEcV } \\
\mathbf{( \% )}\end{array}$ \\
\hline Ficus benjamina & 279 bands & 3 & 0.95 & 11.54 \\
Azalea japonica & 279 bands & 4 & 0.85 & 14.93 \\
Euonymus japonicus & 279 bands & 3 & 0.86 & 22.54 \\
Buxus sempervirens & 279 bands & 3 & 0.67 & 31.23 \\
\hline Pooled data & 279 bands & 3 & 0.69 & 34.99 \\
\hline
\end{tabular}

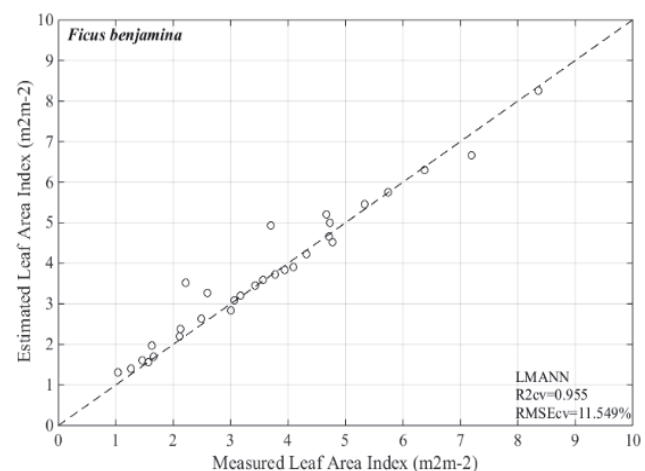

(a)

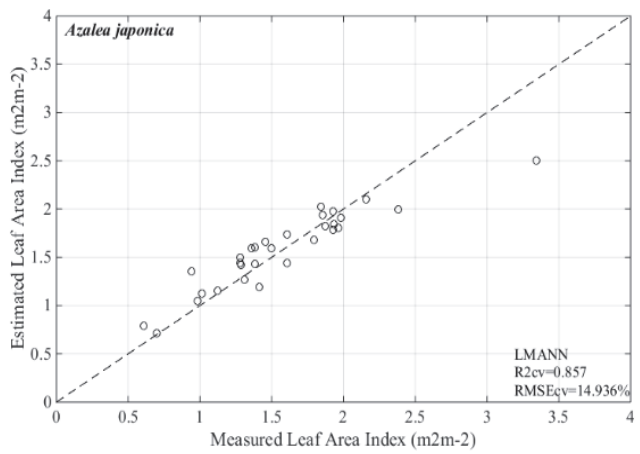

(b) 


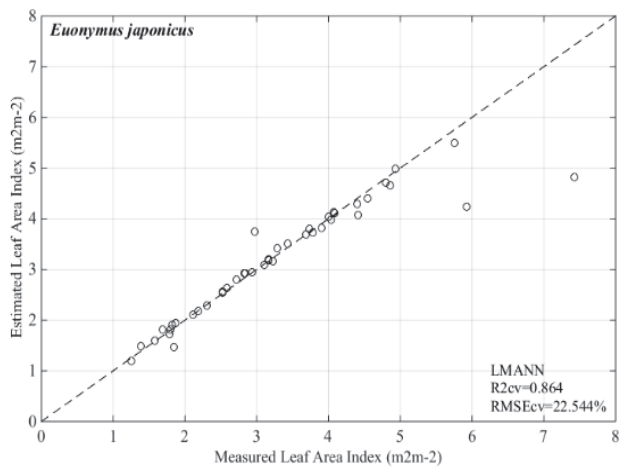

(c)
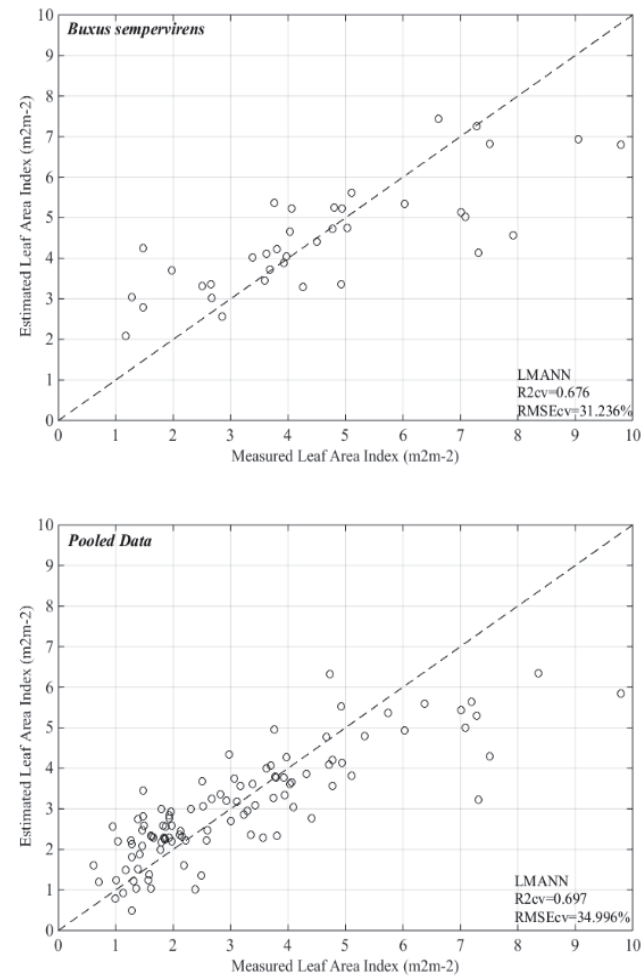

(e)

Figure 3.6: Scatterplot of measured versus estimated leaf area index using LMANN training algorithm with 279 wavebands as input data from 8-14 $\mu \mathrm{m}$ : Ficus benjamina (a), Azalea japonica (b), Euonymus japonicus (c), Buxus sempervirens (d), and pooled data (e).

\subsection{Discussion}

Although it has long been believed that plants are featureless in the TIR region (Wong and Blevin 1967), recent studies have put an end to this assertion and 
showed that different vegetation types have characteristic emissivity signature (French et al. 2000; Ribeiro da Luz and Crowley 2007; Ullah et al. 2012a). This may partly be explained by the lack of appropriate devices to measure radiance or emissivity in the TIR region. This study demonstrates that the biophysical properties of vegetation at the canopy level, such as LAI, can also be retrieved using canopy emissivity spectra in the $8-14 \mu \mathrm{m}$ region. The differences observed in the relationships between LAI and canopy emissivity spectra for individual wavebands as well as the dissimilarity in the position of maximum correlations value can be attributed to differences in canopy structure, and probably by the biochemical concentrations of the leaves of these species. These results highlight the fact that a single thermal waveband does not provide sufficient information to explain a biophysical variable such as LAI. Narrow band indices utilizing information from two-waveband combinations showed higher sensitivity to LAI variability than individual spectral bands, which resulted in a higher $\mathrm{R}^{2}$ with LAI values.

The results demonstrate that like the narrow band indices used in the optical domain for LAI estimations (Darvishzadeh et al. 2008a; Schlerf et al. 2005), the narrow band combinations from the TIR region can also be utilized to retrieve LAI values. Given the results of our experiments, we conclude that vegetation indices can accurately predict LAI for single species in the 8-14 $\mu \mathrm{m}$ thermal region, but not for pooled data of four plant species. The emissivity of the TIR spectra may respond to the effects of leaf surface composition (i.e. cellulose and cutin) and abundance (e.g. thickness of the cutin layer), on the absorption of TIR energy into leaves (Ribeiro da Luz and Crowley 2007), as well as canopy structure (i.e. leaf size and orientation) on radiance scattering and emissivity spectra (Salisbury 1986). In addition, different plant species have a different leaf angle distribution, and as discussed by Sellers (1985), this results in the emissivity spectra and LAI being affected by the leaf angle distribution. Also, the angular effects on emissivity spectra with plants having different proportions of leaves have been demonstrated (Sobrino et al. 2005). Another factor potentially compounding the results is vegetation water content, which has a significant effect on the emissivity spectra of plants through changes in cuticle thickness (Buitrago et al. 2016). Since our plants were well watered the effect of changes in vegetation water content, have not been considered. Additional effort needs are required to 
understand the effect of variation in vegetation water content and physiological condition on the predicting LAI using TIR emissivity spectra.

As noted in Table 3.3, for individual species the best performing thermal narrow band index was similar in all investigated indices (i.e. SD, SR, and ND). However, the best performing thermal narrow band index differed, depending on species. This can again be attributed to differences in the biochemical and structural properties of the species studied. Obtaining similar results for the investigated indices could be due to the fact that our measurements were carried out under controlled laboratory conditions, so atmospheric or sensor errors were considerably reduced. Compared with the narrow band indices all PLSR models achieved relatively high accuracies. This may be due to the fact that (two waveband) vegetation indices applied only a limited amount of the total emissivity information available, whereas the PLSR technique utilized all available spectral wavebands. Our results are in agreement with the finding of Darvishzadeh et al. (2008b), who showed that compared with narrowband vegetation indices (NDVI: $\mathrm{R}^{2}=0.60$, $\mathrm{RMSE}_{\mathrm{CV}}=0.34, \mathrm{~m}^{2} \mathrm{~m}^{-2}$ ), PLSR (utilizing all available spectral bands) improved the estimation of LAI $\left(\mathrm{R}^{2}=0.69, \mathrm{RMSE}_{\mathrm{CV}}=0.32, \mathrm{~m}^{2} \mathrm{~m}^{-2}\right)$ in the optical domain. Moreover, in both methods (i.e. PLSR and narrow-band vegetation indices), the relationship between measured and estimated LAI for pooled data was poor, whereas previously LAI estimated with intermediate accuracy for pooled data from VNIR hyperspectral data. For instance, Schlerf et al. (2005) showed that LAI was predicted with reasonable accuracy from hyperspectral remote sensing data $\left(\mathrm{R}^{2}=0.67, \mathrm{RMSE}=21, \%\right)$.

When the ANN was used, the LAI retrieved was more accurate, and the estimates were much more accurate than those obtained using PLSR and narrow-band vegetation indices. Although a previous study had demonstrated that LAI retrieved from the visible reflectance data when using ANN was underestimated $(\mathrm{RMSE}=0.47)$, especially for LAI higher than $5\left(\mathrm{~m}^{2} \mathrm{~m}^{-2}\right)$ (Bacour et al. 2006), in our study, the ANN technique was the most accurate method for estimating LAI remotely from TIR hyperspectral data even at high LAI values. The comparison between the statistical techniques applied in this study suggests that ANN significantly ( $p$-value $=0.015$ ) improved estimation of LAI in the TIR region $\left(\mathrm{R}^{2}=0.95, \mathrm{RMSE} \mathrm{CV}=11.54, \%\right)$ compared to narrow band indices $\left(\mathrm{R}^{2}=0.65, \mathrm{RMSE} C \mathrm{CV}=31.95, \%\right)$. Although emissivity spectra in the TIR region had a strong logarithmic relation with NDVI in the study by 
Van de Griend and Owe (1993), here, the relation between predicted and measured LAI was linear for all models. This study showed that LAI could be predicted reasonably accurately in the TIR region and that it can be estimated from VNIR/SWIR data slightly more accurately than from TIR data. However, from the scatter plots of paired waveband combinations TIR region it is clear that saturation did not occur in some species (e.g. Ficus benjamina), not even at the relatively high value of $\mathrm{LAI}\left(\mathrm{LAI} \geq 5.5, \mathrm{~m}^{2} \mathrm{~m}^{-2}\right.$ ) (Figures 3.43.6). There is some saturation for Buxus sempervirens around LAI values between 8 and $10\left(\mathrm{~m}^{2} \mathrm{~m}^{-2}\right)$, although saturation still is not observed with a LAI value of $\sim 5.5\left(\mathrm{~m}^{2} \mathrm{~m}^{-2}\right)$. This is a significant advantage of using TIR rather than the VIS/SWIR data for modeling LAI because LAI saturates at approximately 2.5-5.5 $\left(\mathrm{m}^{2} \mathrm{~m}^{-2}\right)$ in the VIS/SWIR (Asner et al. 2003; Carlson et al. 1990; Chen et al. 1997; Sellers 1987).

\subsection{Conclusions}

The results of this study showed that LAI is retrievable from the emissivity spectra using multivariate and univariate statistical techniques at the TIR region. We further found that all investigated indices were obtained similar results for predicting LAI. The LAI of the four species investigated in this study were retrieved with different accuracy no matter which statistical methods applied. In all the models investigated, a linear relationship was found between estimated and measured LAI. The method that most improved the estimation of LAI was the ANN. It is concluded that LAI can successfully be retrieved from TIR hyperspectral data, even at relatively high values of LAI. This study demonstrates proof of concept for measuring LAI using the TIR and brings a following interesting challenge: how to upscale the results from the laboratory (plant canopy) to field conditions. 


\section{Chapter 4}

\section{Retrieving Vegetation Canopy Water Content from Hyperspectral Thermal Measurements*}

* This chapter is based on:

Neinavaz, E., Skidmore, A. K., Darvishzadeh, R., \& Groen, T. A. Retrieving Vegetation Canopy Water Content from Hyperspectral Thermal Measurements. Agriculture and Forest Meteorology. (In review, after revision).

Neinavaz, E., Skidmore, A. K., Darvishzadeh, R., \& Groen, T. A. Vegetation Water Content Retrieval using Thermal Hyperspectral Data. 10 $0^{\text {th }}$ EARSel SIG Imaging Spectroscopy Workshop, 19-21 April 2017, Zurich, Switzerland. 


\begin{abstract}
The retrieval of vegetation canopy water content using thermal hyperspectral (TIR, 8-14 $\mu \mathrm{m}$ ) measurements is investigated in this study. Vegetation water content indicators such as fuel moisture content (FMC, \%, mass-based) and equivalent water thickness (EWT, $\mathrm{g} \mathrm{cm}^{-2}$, area based) play significant roles in plant physiology, as well as in the modelling of fire risk and behavior, particularly in forests. Although retrieval of these parameters, in particular, EWT, has been demonstrated from optical and TIR measurements, to our knowledge their prediction at canopy level in the thermal part of the electromagnetic spectrum has not yet been investigated. Therefore, the application of hyperspectral TIR data for predicting FMC and EWT parameters at canopy level is explored here. The emissivity of spectral data in the TIR region is measured for four species (Azalea japonica, Buxus sempervirens, Euonymus japonicus, and Ficus benjamina) under controlled laboratory conditions, using a portable MIDAC Fourier transform infrared spectrometer. EWT, FMC, and their corresponding canopy emissivity measurements are assessed by destructive sampling of the leaves. Leaf area, as well as fresh and dry mass of the harvested leaves, is determined for all four species. Partial least square regression and artificial neural networks, using various spectral subsets, are used to predict the two variables of interest. Higher estimation accuracies have been obtained for both FMC and EWT at canopy level using artificial neural networks. Unexpectedly, the FMC at canopy level, as a mass-based variable, more accurately retrieved using either method. This is contrary to previous findings using multispectral and hyperspectral data. Our results suggest that plant mass may play a greater role in determining spectral emissivity than plant area does.
\end{abstract}




\subsection{Introduction}

In the past decades, remote sensing has been recognized as a tool to monitor and quantify vegetation variables. Canopy biophysical and biochemical variables involved in biophysical processes of terrestrial ecosystems (e.g. carbon and water cycling) determine how plants respond to environmental factors and influence ecosystem processes as well as responses to climate change. Vegetation water content is an indication of vegetation physiological condition and terrestrial ecosystem status (Claudio et al. 2006). It plays a significant role in the understanding of the earth's ecosystem functioning (Clevers and Kooistra 2006) when assessing drought status in forestry and agriculture (Peñuelas and Filella 1998), or estimating wildfire occurrence (Chuvieco et al. 2004). For instance, biochemical processes (e.g. photosynthesis and evaporation) are limited by vegetation water content (Peñuelas et al. 1994). Flammability in a forest, a critical parameter in fire ignition, also depends on vegetation water content (i.e. Fuel moisture content (FMC) in forest fire literature). The principal indicators used in remote sensing that characterize the amount of water in vegetation are fuel moisture content (FMC, \%) and equivalent water thickness (EWT, $\mathrm{g} \mathrm{cm}^{-2}$ ) (Colombo et al. 2008). EWT is the amount of water per unit leaf area (Clevers et al. 2010; Hunt and Rock 1989; Jacquemoud and Baret 1990). In remote sensing studies, EWT is at times also expressed as vegetation liquid water content (Cheng et al. 2006). FMC can be defined as the ratio between the water quantity in the fuel (the difference between fresh and dry weight) and either the fresh weight or the dry weight (Ceccato et al. 2001; Danson et al. 1992). FMC is highly correlated with risk of wildfire (Chuvieco et al. 2004; Hunt Jr et al. 2013) and is described as leaf water content or leaf gravimetric water content in remote sensing studies (Buitrago et al. 2016; Ceccato et al. 2002a; Ceccato et al. 2001; Colombo et al. 2008; Ullah et al. 2014). In this study, EWT and FMC are considered to be indicators for area-based and mass-based vegetation water content, respectively. Area-based variables are valuable from a plant physiological point of view. Transpiration, respiration, gas exchange (e.g. uptake of $\mathrm{CO}_{2}$ and $\mathrm{H}_{2} \mathrm{O}$ ), as well as plant physiochemical processes of photosynthesis (e.g. carbon fixation), occur as a flux per unit leaf surface area (Hikosaka 2004; Lloyd et al. 2013). Mass-based parameters are important for estimating carbon flux, as well as growth in leaves. 
In the past decades, retrieval of these variables from remote sensing data was investigated using a broad range of approaches (Gao 1996; Gao and Goetzt 1995; Serrano et al. 2000). The majority of these studies focused on the visible, near-infrared (VNIR, 0.3-1.0 $\mu \mathrm{m}$ ), and shortwave-infrared (SWIR 1.0-2.5 $\mu \mathrm{m}$ ) regions (Ali et al. 2015; Ceccato et al. 2002a; Clevers and Kooistra 2006; Clevers et al. 2010; Dawson et al. 1999; Jin and Liu 1997; Kalaitzidis et al. 2010; Laurin et al. 2014; Mirzaie et al. 2014; Qi et al. 2014; Tucker 1980; Ustin et al. 1998; Yebra et al. 2013), as well as on mid-wave infrared (2.5-6 $\mu \mathrm{m})$ (Ullah et al. 2013). Estimation of EWT and FMC using remote sensing data has posed different challenges. Verbesselt et al. (2007) and Chuvieco et al. (2003) state that operational estimation of EWT is difficult in the field because it requires the calculation of leaf area; while Jacquemoud and Baret (1990) demonstrate that it is a challenge to predict FMC due to the difficulty in calculating vegetation dry matter content $\left(\mathrm{DMC}, \mathrm{g} \mathrm{m}^{-2}\right)$. Despite the fact that both EWT and FMC provide information on the amount of water present in vegetation, estimation of EWT using empirical and physical models has been more successful than FMC when using remote sensing data (Colombo et al. 2008). Moreover, it has been demonstrated that EWT relates better to spectral reflectance measurement (Ceccato et al. 2002b; Danson and Bowyer 2004) and its estimation is less problematic than FMC's from remote sensing data in the VNIR and SWIR regions (Colombo et al. 2008; Danson and Bowyer 2004). In contrast, it is hard to generalize the relationship between FMC and spectral reflectance as the found relationships in the optical domain (VNIR/SWIR) differ in previous studies (Chuvieco et al. 2003; Chuvieco et al. 2002; Paltridge and Barber 1988; Pinol et al. 1998). In this respect, using thermal infrared hyperspectral data (TIR, 8-14 $\mu \mathrm{m}$ ) opens new opportunities for estimating FMC for vegetation studies, particularly in forestry applications to model the fire risk.

To our knowledge, spectral emissivity from hyperspectral thermal data has been poorly studied in the past and has not yet been used to retrieve EWT and FMC from multiple plant species at canopy level. A few studies have attempted to relate emissivity spectra in the TIR domain to vegetation water content at leaf level using hyperspectral TIR data (Buitrago et al. 2016; Ullah et al. 2012b). Recently, Ullah et al. (2014) revealed that the MIR domain is a highly sensitive spectral region for estimating water content at the leaf level, using narrow band indices developed from hyperspectral MIR and TIR data. 
Their findings show that the optimal narrow-band indices in the TIR region are the wavebands $11.31 \mu \mathrm{m}$ and $11.46 \mu \mathrm{m}$.

Additionally, little is known about properties of vegetation in the TIR region, while the TIR offers a wide atmospheric window with large transparency which makes this region suitable for remote sensing studies (Clerbaux et al. 2011). Remote sensing data from the VNIR-SWIR alone is not sufficient to explain the structural and chemical characteristics of vegetation. For instance, the spectral features resulting from the combination of primary absorption features in the VNIR and SWIR regions, make it challenging to link particular wavebands to an individual chemical constituent, due to overlapping absorption features of plant biochemical contents (Kokaly et al. 2009). Moreover, the spectral profiles of plant species in the VNIR and SWIR regions are mostly similar in shape because reflectance spectra in these regions are conquered by absorption features of chemical components weighted by their concentrations and to a great extent, masked by water (Kokaly and Clark 1999). In contrast, recent studies in the TIR domain demonstrated that leaf water content is the most important parameters associated with variations in TIR spectra (Buitrago et al., 2017), and also that the response of emissivity spectra (in particular for fresh leaves) are linked to structure and thickness of cuticle as well as leaf water content (Buitrago et al. 2016).

To scale EWT and FMC up from leaf level up to canopy level requires considering leaf area index (LAI, $\mathrm{m}^{2} \mathrm{~m}^{-2}$ ) as an indispensable variable. This study considers both EWT and FMC variables at canopy level (hereafter referred to as $\mathrm{EWT}_{\mathrm{C}}$ and $\mathrm{FMC}_{\mathrm{C}}$, respectively) and reflects on the applicability of emissivity spectra from hyperspectral TIR data to estimate the $\mathrm{EWT}_{\mathrm{C}}$ and $\mathrm{FMC}_{\mathrm{C}}$, using partial least square regression (PLSR) and artificial neural networks (ANN). To estimate these variables from emissivity spectra in the TIR region, we conducted a laboratory experiment, measuring emissivity spectra for four plant species in the thermal infrared between $8 \mu \mathrm{m}$ and $14 \mu \mathrm{m}$. 


\subsection{Materials and methods}

\subsubsection{Vegetation measurements}

Four different plant species were selected: Azalea japonica (Azalea) $(\mathrm{n}=10)$, Buxus sempervirens (Common box) $(\mathrm{n}=10)$, Euonymus japonicus (Japanese spindle) $(\mathrm{n}=11)$, and Ficus benjamina (Weeping fig) $(\mathrm{n}=6)$. To create variation in the variables values of interest (i.e. $\mathrm{EWT}_{\mathrm{C}}$ and $\mathrm{FMC}_{\mathrm{C}}$ ) and their corresponding canopy emissivity, leaves from different layers of the canopy were randomly removed in 3 to 4 consecutive steps (depending on the plant size) hence after each removal phase, the total value of the considered variables was lowered. The destructive sampling of the 37 plants thus yielded 144 estimates for both $\mathrm{EWT}_{\mathrm{C}}$ and $\mathrm{FMC}_{\mathrm{C}}$. The leaf harvesting was executed within a couple of hours to minimize possible changes in the plant's physiological status, such as stomatal conductance, and to capture the potential interference of different physiological processes as a result of these defoliations. The leaf area of the harvested leaves was measured using a LI$3000 \mathrm{C}$ portable leaf area meter (LICOR, NE, USA).

\subsubsection{Calculation of vegetation water indicators}

The mass of the freshly harvested leaves from each sample was precisely weighed, using a digital weight balance with a $100 \mu \mathrm{g}$ accuracy, immediately after harvesting. Leaves were then oven-dried at $75^{\circ} \mathrm{C}$ for 48 hours until a constant weight was attained. EWT was computed using the following equation (Colombo et al. 2008):

$E W T=\frac{W_{f}-w_{d}}{A \times P_{w}}$

where $w_{f}$ and $w_{d}$ stand for a given sample's fresh and dry mass, respectively, $A$ is sample leaf area and, $P_{w}$ is a physical constant representing the density of pure water $\left(1 \mathrm{~g} \mathrm{~cm}^{-3}\right)$.

LAI is the one-sided leaf area $\left(\mathrm{m}^{2}\right)$ per unit of horizontal surface area $\left(\mathrm{m}^{2}\right)$ (Watson 1947). To calculate the LAI $\left(\mathrm{m}^{2} \mathrm{~m}^{-2}\right)$, the measured surface areas of the leaves $\left(\mathrm{m}^{2}\right)$ were divided by the corresponding ground area of the canopy $\left(\mathrm{m}^{2}\right)$. By multiplying EWT with the LAI, the total canopy water content per unit of ground area (EWTC, $\mathrm{g} \mathrm{cm}^{-2}$ ) is obtained (Clevers et al. 2010; Yebra et al. 2013): 
EWT and FMC refer to two different quantities and describe vegetation water content in a different way (Ceccato et al. 2001; Ghulam et al. 2007). Several methods have been proposed to measure FMC.

Since FMC is controlled and determined by EWT and dry matter content (DMC) according to $F M C=E W T / D M C$ (Jacquemoud and Baret 1990), Riaño et al. (2005) predicted FMC by separately predicting EWT and DMC. They assumed vegetation DMC to be constant throughout the year. However, this assumption is not necessarily valid, particularly during drought periods. Garnier et al. (2001), observed that vegetation DMC decreased during a period of drought due to reduced productivity. In addition, these variables (i.e. EWT and DMC) are entirely independent of each other. Therefore, in this study, the most common equation (3) which is used by many researchers (Chuvieco et al. 1999; Desbois et al. 1997) is applied to calculate FMC. For scaling up of FMC, Hunt et al. (2012) estimated $\mathrm{FMC}_{\mathrm{C}}$ by multiplying leaf EWT and DMC by LAI. In this respect, $\mathrm{FMC}_{\mathrm{C}}$ is estimated by multiplying FMC by LAI.

$F M C=\frac{W_{f}-W_{d}}{W_{f} \text { or } W_{d}} \times 100$

$F M C c=\frac{W_{f}-W_{d}}{W_{f} \text { or } W_{d}} \times L A I$

\subsubsection{Laboratory conditions}

To create optimal measurement conditions and reduce any possible error from changing atmospheric conditions or temperature, measurements were carried out under controlled laboratory conditions, with walls, ceiling, and ground coated with a black material (Avis Aqua Blackboard Black) and plastic of known emissivity. The traditional procedure when measuring emissivity is to heat samples (e.g. geological samples) to a temperature above the ambient condition to create a thermal contrast (Ribeiro da Luz and Crowley 2007; Salisbury 1998). However, such treatment stresses plant samples, so instead we held the laboratory temperature at $10^{\circ} \mathrm{C}$, thus generating a suitable thermal contrast with the plants, which were kept at room temperature $\left(20^{\circ} \mathrm{C}\right)$ and only briefly (individually) transferred to the cool laboratory room to make the spectral measurements. Because Cheng et al. (2006) revealed that $\mathrm{EWT}_{\mathrm{C}}$ is 
under-estimated in a semi-arid ecosystem due to a significant proportion of the soil background being mistakenly included as canopy closure, the background soil was covered with black plastic of known emissivity to minimize possible soil effects. We measured the emissivity spectra of the black plastic using a BRUKER Vertex 70 laboratory FTIR spectrometer. The black plastic had low emissivity and was assumed to cause minimal interference with the thermal radiance measurements of the samples $(60 \mathrm{~cm})$.

\subsubsection{Canopy spectroscopic measurement}

\subsection{Thermal infrared emission spectroscopy}

The radiance spectra were measured using a portable FTIR spectrometer (Model M4401-F; MIDAC Corporation, CA, USA). The MIDAC configuration enables measurement of radiance spectra within the spectral range of $2.5 \mu \mathrm{m}$ to $20 \mu \mathrm{m}$ with an adjustable spectral resolution of 32 to 0.5 $\mathrm{cm}^{-1}$ (Eisele et al. 2015). The MIDAC has a liquid-nitrogen-cooled MercuryCadmium-Telluride (MCT) detector and customized foreoptics that consist of a flat folding mirror on a rotational axis, which allows measurements of two internal blackbodies (hot and cold) for calibration and measuring each sample. The MIDAC's folding mirror was kept at nadir position above the samples. The MIDAC's field of view (using a cut-off point of 5\% of the maximum responsivity) has a starting diameter of $53 \mathrm{~mm}$ at the folding mirror and spreads with about $18 \mathrm{mrad}$. Since the amount of thermal emission varies according to the distance between the sample and the sensor (Ribeiro da Luz and Crowley 2007), and in order to reduce atmospheric attenuation (Korb et al. 1996), measurements were made with a fixed vertical distance between sensor and sample.

\subsection{Radiometric calibration and down-welling radiance measurements}

The emissivity spectra of plant canopies were obtained using a series of FTIR measurements performed in the following order: radiance measurements of the hot blackbody, radiance measurement of the cold blackbody, radiance measurement of the sample (the plant canopy), and finally, radiance measurements of a highly diffuse reflecting gold plate $\left(\right.$ Infragold $\left.^{\circledR}\right)$. The temperature of the hot and cold blackbodies was checked between the measuring of each sample. The cold blackbody temperature was set just below 
the ambient temperature, at $5^{\circ} \mathrm{C}$ (Korb et al. 1996). The hot blackbody temperature was set above the sample temperature, at $30^{\circ} \mathrm{C}$ (Hori et al. 2006; Salvaggio and Miller 2001). Details of the radiometric calibration of the blackbodies radiances can be found in Hook and Kahle (1996). A diffuse reflecting gold plate with an emissivity of $\sim 0.04$ was used to measure downwelling radiance (DWR) to correct radiance measurements and determine any significant influence of laboratory background emissions (Eisele et al. 2015). The infragold plate was placed directly under the MIDAC sensor at the same distance as the sample. The temperature of the sample, the infragold plate, and the laboratory was frequently monitored before and after each measurement, using thermistors (FLUKE 51 II Thermometer and Precision IR Thermometer) to detect any possible changes in temperature. Such changes in temperature could disturb the thermal contrast and result in over- or underestimation of the emissivity values. The measurement series took less than five minutes each to minimize possible temperature drift of the instrument, physiological changes in the plants, and fluctuations in laboratory temperature (Hori et al. 2006).

\subsubsection{Canopy radiance measurements}

The radiance spectra of the plant canopies were measured at wavelengths of $2.5 \mu \mathrm{m}$ and $20 \mu \mathrm{m}$ with a resolution of $2 \mathrm{~cm}^{-1}$. On average, 32 scans were performed for each sample. The canopy emissivity measurements included 279 wavebands between $8 \mu \mathrm{m}$ and $14 \mu \mathrm{m}$. Measurements outside this range had the very low signal strength and were therefore excluded from further analysis. After each set of measurements, the canopy was rotated 90 degrees clockwise, and the series of measurements was repeated until the canopy was fully rotated. The final corresponding canopy emissivity spectra of each sample for a particular value of $\mathrm{FMC}_{\mathrm{C}}$ and $\mathrm{EWT}_{\mathrm{C}}$ was then calculated from the average of four sets of measurements (covering 360 degrees). The position of the MIDAC sensor above the canopy was kept constant. In total, 576 $(4 \times 144)$ canopy radiance measurements were obtained from the four plant species.

\subsubsection{Data pre-processing}

Spectral emissivity of the plants was calculated from their absolute radiance using the following equation (Korb et al. 1996), 
$\varepsilon_{\text {sam }}(\lambda)=\frac{L_{\text {sam }}(\lambda)-\mathrm{L}_{\text {DWR }}(\lambda)}{\mathrm{B}\left(\lambda, \mathrm{T}_{\mathrm{sam}}\right)-\mathrm{L}_{\mathrm{DWR}}(\lambda)}$

where $\varepsilon_{\text {sam }}(\lambda)$ denotes the directional emissivity of the sample at wavelength $\lambda, L_{\text {sam }}(\lambda)$ is the spectral radiance from the target, $\mathrm{T}_{\text {sam }}$ is the actual physical temperature of the sample, $\mathrm{B}\left(\lambda, \mathrm{T}_{\mathrm{sam}}\right)$ is the Planck function at wavelength $\lambda$ and the sample temperature, and $\mathrm{L}_{\mathrm{DWR}}(\lambda)$ is the total spectral DWR from the hemisphere above the sample. To retrieve canopy surface emissivity, the information regarding the precise surface temperature $\left(\mathrm{T}_{\text {sam }}\right)$ at the time of the measurement is essential. Therefore, despite measuring canopy temperature before and after each measurement, the blackbody fit method was used to estimate the exact sample surface temperature value at the time of measurement. Details on the blackbody fit method can be found in Kahle and Alley (1992) and Salvaggio and Miller (2001). A Savitzky-Golay filter with a frame size of 15 data points and second- degree polynomial was used to reduce the noise in the canopy emissivity spectra (Savitzky and Golay 1964). Data were analyzed and processed using MATLAB R2013b (Mathwork, Inc).

\subsubsection{Estimation of variables}

\subsubsection{Partial least square regression}

Hyperspectral data have a high dimensionality and also a high degree of collinearity in adjacent wavebands. This challenge is somewhat overcome using partial least squares regression (PLSR), one of the most used multivariate statistical techniques in hyperspectral studies, which transforms the variables into new orthogonal (Abdel-Rahman et al. 2014; Abdi 2010; Chin et al. 2003). PLSR has been broadly applied as a multivariate quantitative technique in remote sensing vegetation analysis (Cho et al. 2007; Kooistra et al. 2004). It is a successful empirical approach to derive biochemical and biophysical properties from canopy spectral data in the VNIR (Asner and Martin 2008; Darvishzadeh et al. 2008b; Mirzaie et al. 2014), as well as biochemical and biophysical variables at both leaf and canopy level from emissivity spectra in the TIR region (Neinavaz et al. 2016c; Ullah et al. 2014). It is a "full spectrum" method, which has the advantage that it makes use of all available spectral wavelengths (Haaland and Thomas 1988). Here, the PLSR analysis was used to determine the relative contribution of the 279 wavebands of the canopy emissivity spectra 
(independent variables) to explain variation in $\mathrm{EWT}_{\mathrm{C}}$ and $\mathrm{FMC}_{\mathrm{C}}$ for all the sampled species. The canopy emissivity spectra as independent variables were mean-centered before performing the PLSR analysis. An optimal number of factors was determined to avoid overfitting and to prevent collinearity, using cross-validation procedures with the root mean squared error (RMSEcv) as a quality indicator. An optimal number of factors indicates the number of the extracted factor for which the total prediction error is minimized. A cross-validation procedure, also known as the "leave one out method" (Duda and Hart 1973), is a method for selecting a model according to its predictive ability (Shao 1993). In cross-validation each data point is successively "left out" from the data and used as the validation set. The criterion for adding an extra factor to the model was that it would decrease the

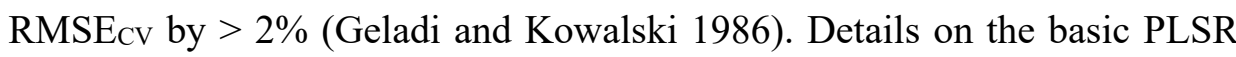
algorithm can be found in Geladi and Kowalski (1986) and Williams and Norris (1987). All PLSR analyses were carried out using the TOMCAT toolbox 1.01 within MATLAB (Daszykowski et al. 2007). Since $\beta$ coefficients represent the contribution of each waveband to the model, they were utilized to determine the importance of wavebands in each fitted PLSR model. The thresholds for $\beta$ coefficients were specified according to their standard deviations. Wavebands with a $\beta$ coefficient above this threshold were considered to be contributing significantly. The sign of the $\beta$ coefficients (i.e. plus or minus) represents the direction of the relationship between emissivity value (i.e. independent variable) and $\mathrm{FMC}_{\mathrm{C}}$ and $\mathrm{EWT}_{\mathrm{C}}$ (i.e. dependent variable). The higher absolute value of $\beta$ coefficients represents the stronger relation.

\subsubsection{Artificial neural networks}

The use of artificial neural networks (ANN) has been shown to be an effective alternative to more traditional statistical techniques (Paliwal and Kumar 2009). Recently, Neinavaz et al. (2016c) demonstrated the significance of using ANN for the prediction of LAI using hyperspectral TIR data. The multilayer perceptron is a broadly utilized neural network in remote sensing studies (Atzberger 2004; Mirzaie et al. 2014). A typical ANN consists of different types of layers, namely: input layers, hidden layers, and output layers. Three different data sets were employed as input layer, comprising (1) all available canopy emissivity values in 279 wavebands between $8 \mu \mathrm{m}$ and $14 \mu \mathrm{m}$ 
(labelled as ANW); (2) the most important wavebands which were identified from PLSR analysis (labelled as IPW); (3) the best performing narrow bands as determined by Ullah et al (2014) (labelled as UIPW). For network training, two algorithms were considered: the Levenberg-Marquardt (LMANN) and the Scaled Conjugate Gradient (ScgANN) algorithm, and they were applied as two common training algorithms in back-propagation networks to develop models for the estimation of our variables of interest. Although there are no rules that determine the optimal number of hidden layers, an increase in the number of hidden layers enables the network to tackle more complex problems (Atkinson and Tatnall 1997). For instance, Skidmore et al. (1997) revealed that higher accuracy is obtained with three hidden layers, than with two or one. As the prediction performance of ANN depends on the number of neurons in the hidden layer, the best ANN size was identified by testing different numbers. The early stopping technique was utilized to avoid overfitting problems. In this technique, training stops as soon as performance on a validation data set begins to worsen (Nowlan and Hinton 1992). Furthermore, linear regression analyses were performed between the predicted and the measured values of $\mathrm{EWT}_{\mathrm{C}}$ and $\mathrm{FMC}_{\mathrm{C}}$ to determine the best ANN model. The cross-validation procedure was repeated 1000 times, and results were averaged to reduce unfavorable effects from the random initialization of the optimization routine. The reliability of ANN in estimating our variables was evaluated using the cross-validated coefficient of determination $\left(\mathrm{R}^{2} \mathrm{CV}\right)$ and the cross-validated root mean squared error $\left(\right.$ RMSE $\left._{\mathrm{CV}}\right)$. All analyses were carried out using the MATLAB neural network toolbox.

\subsection{Results}

\subsubsection{Relationship between canopy variables and emissivity spectra}

The measured $\mathrm{EWT}_{\mathrm{C}}$ and $\mathrm{FMC}_{\mathrm{C}}$ values demonstrated a wide range [0.02-0.2 for $\mathrm{EWT}_{\mathrm{C}}$; and 0.51-6.99 for $\mathrm{FMC}_{\mathrm{C}}$ ] due to the variation in the leaf shapes and sizes of the plants. As can be observed from Table 4.1, Azalea japonica has the lowest value for EWT $\mathrm{C}$ and $\mathrm{FMC}_{\mathrm{C}}$, while Ficus benjamina and Buxus sempervirens have the highest values for $\mathrm{FMC}_{\mathrm{C}}$ and $\mathrm{EWT}_{\mathrm{C}}$, respectively. 
Table 4.1: The statistics of the variables measured per plant species. LAI represents the leaf area index $\left(\mathrm{m}^{2} \mathrm{~m}^{-2}\right) ; E W T_{C}$ is the canopy equivalent water thickness $\left(\mathrm{g} \mathrm{cm}^{-}\right.$ $\left.{ }^{2}\right)$, EWT is the equivalent water thickness $\left(\mathrm{g} \mathrm{cm}^{-2}\right), F M C_{C}$ is the canopy fuel moisture content, and FMC is the fuel moisture content (\%).

\begin{tabular}{|c|c|c|c|c|c|c|c|}
\hline \multirow{2}{*}{ Species } & \multirow{2}{*}{ Variables } & \multirow{2}{*}{ Min } & \multirow{2}{*}{ Max } & \multicolumn{2}{|c|}{ Mean } & \multirow{2}{*}{$\begin{array}{c}\text { Std. } \\
\text { Deviation }\end{array}$} & \multirow{2}{*}{$\begin{array}{c}\text { Sample } \\
\text { size }\end{array}$} \\
\hline & & & & Statistic & SD. Error & & \\
\hline \multirow{5}{*}{ 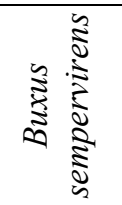 } & LAI & 1.17 & 9.80 & 4.54 & 0.34 & 2.14 & \multirow{5}{*}{30} \\
\hline & $\mathrm{EWT}_{\mathrm{C}}$ & 0.03 & 0.20 & 0.10 & 0.00 & 0.04 & \\
\hline & EWT & 0.01 & 0.08 & 0.02 & 0.00 & 0.01 & \\
\hline & $\mathrm{FMC}_{\mathrm{C}}$ & 0.79 & 5.09 & 3.04 & 0.26 & 1.32 & \\
\hline & FMC & 46.54 & 84.58 & 69.21 & 1.77 & 8.68 & \\
\hline \multirow{5}{*}{ 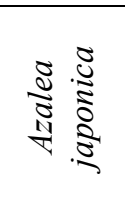 } & LAI & 0.60 & 3.35 & 1.57 & 0.09 & 0.54 & \multirow{5}{*}{30} \\
\hline & $\mathrm{EWT}_{\mathrm{C}}$ & 0.02 & 0.08 & 0.04 & 0.00 & 0.01 & \\
\hline & EWT & 0.02 & 0.04 & 0.03 & 0.00 & 0.00 & \\
\hline & $\mathrm{FMC}_{\mathrm{C}}$ & 0.51 & 2.60 & 1.25 & 0.07 & 0.42 & \\
\hline & FMC & 71.00 & 80.42 & 75.32 & 0.51 & 2.81 & \\
\hline \multirow{5}{*}{ 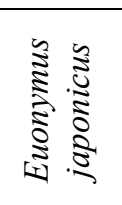 } & LAI & 1.25 & 7.43 & 3.28 & 0.19 & 1.32 & \multirow{5}{*}{44} \\
\hline & $\mathrm{EWT}_{\mathrm{C}}$ & 0.08 & 0.16 & 0.11 & 0.00 & 0.02 & \\
\hline & EWT & 0.03 & 0.05 & 0.03 & 0.00 & 0.00 & \\
\hline & $\mathrm{FMC}_{\mathrm{C}}$ & 1.43 & 3.68 & 2.37 & 0.15 & 0.68 & \\
\hline & FMC & 74.79 & 80.18 & 78.46 & 0.33 & 1.46 & \\
\hline \multirow{5}{*}{ 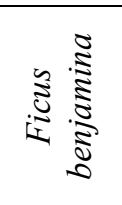 } & LAI & 1.04 & 8.36 & 3.60 & 0.32 & 1.80 & \multirow{5}{*}{30} \\
\hline & $\mathrm{EWT}_{\mathrm{C}}$ & 0.03 & 0.19 & 0.10 & 0.00 & 0.04 & \\
\hline & EWT & 0.2 & 0.5 & 0.03 & 0.00 & 0.00 & \\
\hline & $\mathrm{FMC}_{\mathrm{C}}$ & 0.99 & 6.99 & 3.11 & 0.27 & 1.52 & \\
\hline & FMC & 79.88 & 95.00 & 87.04 & 0.73 & 4.03 & \\
\hline
\end{tabular}

The correlation between EWT and LAI, as well as FMC and LAI, were quantified using the coefficient of determination $\left(\mathrm{R}^{2}\right)$. The results reveal that LAI has a negative correlation with EWT $\left(\mathrm{R}^{2}=0.25\right)$ and FMC $\left(\mathrm{R}^{2}=0.05\right)$. In addition, the result showed that the poor correlation exists between EWT and FMC $\left(\mathrm{R}^{2}=0.20\right)$ which confirms these variables effectively reflecting different properties. 


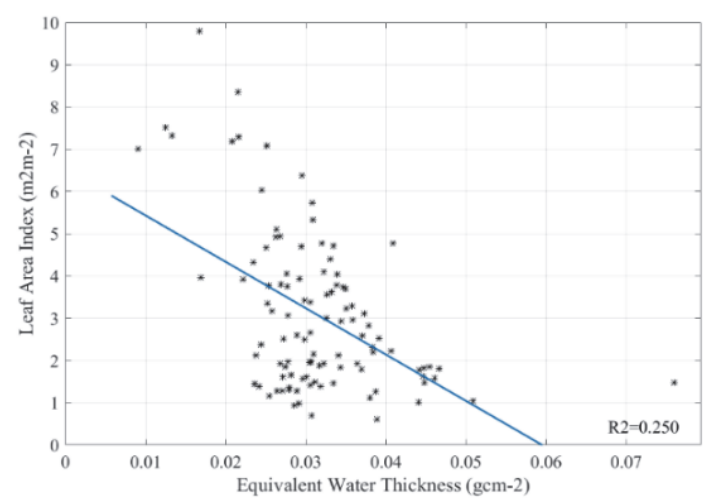

(a)

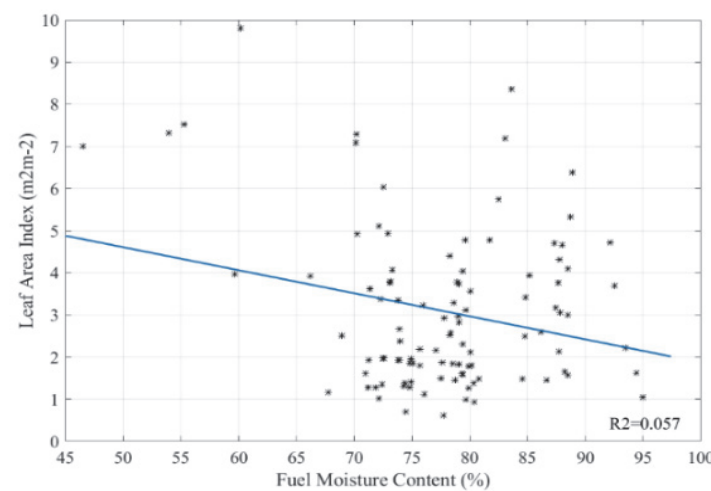

(b)

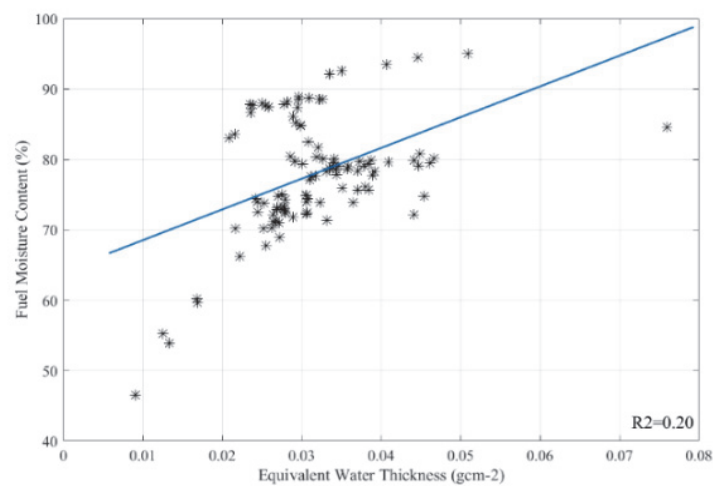

(c)

Figure 4.1: Measured leaf area index $\left(\mathrm{m}^{2} \mathrm{~m}^{-2}\right)$ versus measured equivalent water thickness $\left(\mathrm{g} \mathrm{cm}^{2}\right)(\mathrm{a})$, and fuel moisture content $(\%)(\mathrm{b})$, and measured equivalent water thickness versus measured fuel moisture content $(\mathrm{c})$. 


\subsubsection{Partial least square regression and vegetation water content variables}

The most important wavebands for each species identified in the PLSR model are tabulated in Table 4.2. The number of factors included in each PLSR model ranges from three to five per species. The $\beta$ coefficients associated with the PLSR model for estimation of EWTC and FMC $\mathrm{C}$ across the species and pooled data are shown in Figure 4.2. As can be seen in Table 4.2, among the investigated species $\mathrm{EWT}_{\mathrm{C}}$ and $\mathrm{FMC}_{\mathrm{C}}$ have been accurately retrieved for Azalea japonica and least accurately retrieved for Buxus sempervirens. In addition, the results show that $\mathrm{FMC}_{\mathrm{C}}$ is predicted more accurately than EWTC.

Table 4.2: The performance of partial least square regression for estimating canopy equivalent water thickness $\left(E W T_{C}, \mathrm{~g} \mathrm{~cm}^{-2}\right)$, and canopy fuel moisture content $\left(F M C_{C}\right)$. RMSEcv is the relative cross-validated root mean squared error and $R^{2}$ is the coefficient of determination.

\begin{tabular}{|c|c|c|c|c|c|c|}
\hline Species & Variable & $\begin{array}{c}\text { No. of } \\
\text { Factors }\end{array}$ & $\mathbf{R}^{2}$ & $\mathrm{RMSE}_{\mathrm{CV}}$ & $\begin{array}{c}\text { SD of } \\
\beta \text { Coeff }\end{array}$ & $\begin{array}{c}\text { Important } \\
\text { wavebands }(\mu \mathrm{m})\end{array}$ \\
\hline \multirow{2}{*}{$\begin{array}{c}\text { Ficus } \\
\text { benjamina }\end{array}$} & EWTC $_{C}$ & 3 & 0.67 & 0.02 & 0.09 & $\begin{array}{c}8.8,10.6,10.7,10.8 \\
13.2-13.5\end{array}$ \\
\hline & $\mathrm{FMC}_{\mathrm{C}}$ & 4 & 0.75 & 0.87 & 3.76 & $\begin{array}{c}8.8,11.9,12.0,13.7- \\
14\end{array}$ \\
\hline \multirow{2}{*}{$\begin{array}{c}\text { Azalea } \\
\text { japonica }\end{array}$} & $\mathrm{EWT}_{\mathrm{C}}$ & 5 & 0.81 & 0.00 & 0.03 & $8.0,8.1,8.2,8.8,10.4$ \\
\hline & $\mathrm{FMC}_{\mathrm{C}}$ & 5 & 0.80 & 0.18 & 3.36 & $8.3-8.6,8.8,9.5$ \\
\hline \multirow{2}{*}{$\begin{array}{l}\text { Euonymus } \\
\text { japonicus }\end{array}$} & $\mathrm{EWT}_{\mathrm{C}}$ & 4 & 0.78 & 0.01 & 0.13 & $\begin{array}{c}8.3,8.8,9.6,9.8,9.9, \\
10.4,10.5\end{array}$ \\
\hline & $\mathrm{FMC}_{\mathrm{C}}$ & 3 & 0.80 & 0.44 & 4.41 & $\begin{array}{c}8.1,8.3,8.8,9.6,9.9, \\
10.0,10.3-10.5\end{array}$ \\
\hline \multirow{2}{*}{$\begin{array}{c}\text { Buxus } \\
\text { sempervirens }\end{array}$} & $\mathrm{EWT}_{\mathrm{C}}$ & 4 & 0.58 & 0.02 & 0.08 & $\begin{array}{l}8.0,8.5,8.8,9.2-9.5, \\
11.1,13.5,13.8-14.0\end{array}$ \\
\hline & $\mathrm{FMC}_{\mathrm{C}}$ & 5 & 0.73 & 0.70 & 7.03 & $\begin{array}{c}\text { 8.0, 8.8, 9.0,9.1, 9.3, } \\
\text { 11.1, 12.0-12.3, 12.5, } \\
13.0,13.5\end{array}$ \\
\hline \multirow{2}{*}{ Pooled data } & $\mathrm{EWT}_{\mathrm{C}}$ & 5 & 0.39 & 0.03 & 0.11 & $\begin{array}{c}8.8,9.0,9.4,9.6 \\
10.1,11.4,11.8,11.9, \\
12.3,14.0\end{array}$ \\
\hline & $\mathrm{FMC}_{\mathrm{C}}$ & 5 & 0.42 & 1.07 & 3.77 & $\begin{array}{c}\text { 8.7, 8.8, 9.4-9.7, } \\
10.1,11.1,11.7-11.9, \\
13.7\end{array}$ \\
\hline
\end{tabular}



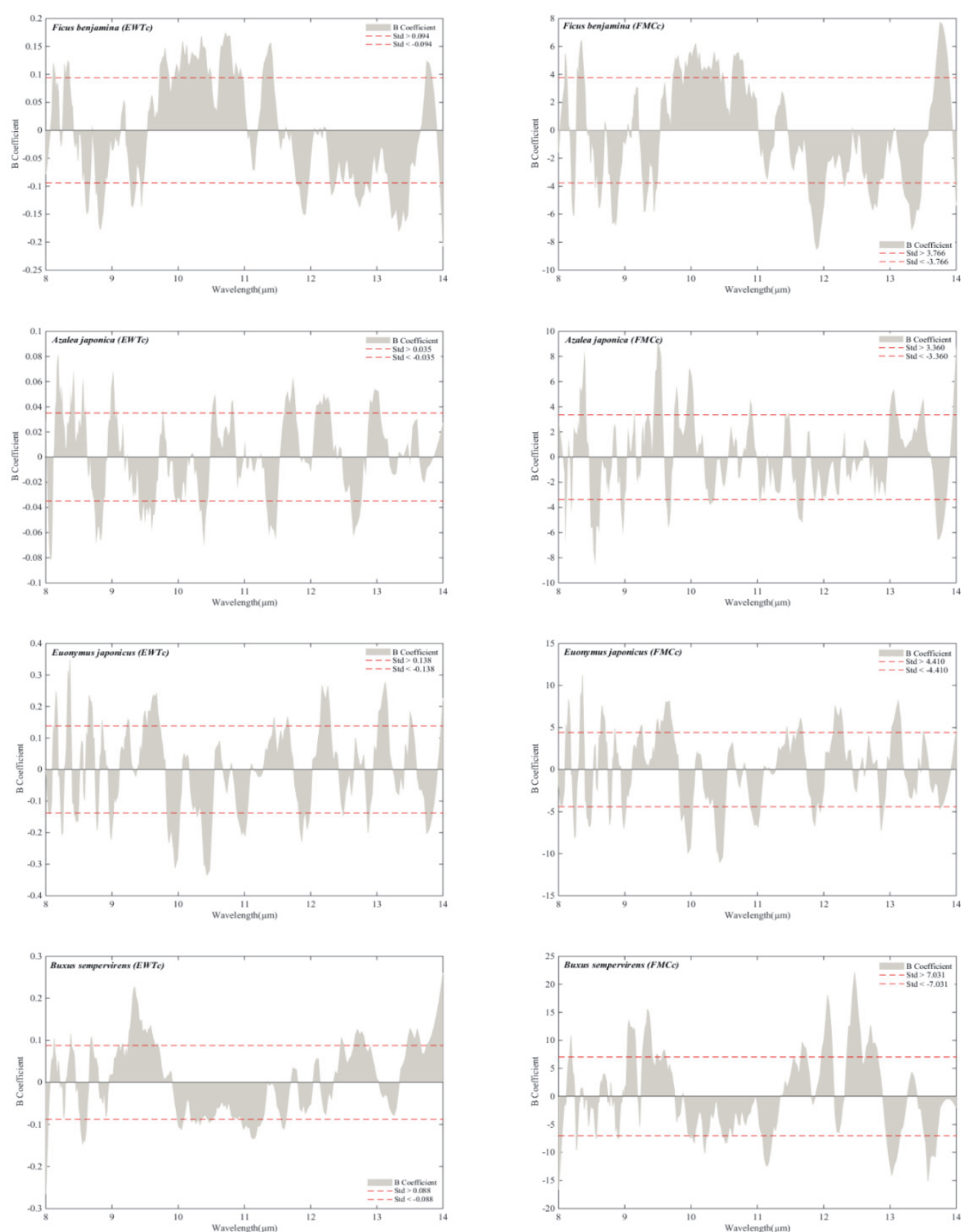

(a)

(b)

Figure 4.2: The $\beta$ coefficients associated with the PLSR model for canopy equivalent water thickness $\left(\mathrm{EWT}_{\mathrm{C}}\right)$ (left side diagrams), and canopy fuel moisture content $\left(\mathrm{FMC}_{\mathrm{C}}\right)$ (right side diagrams) for the four species The threshold is based on the corresponding standard deviation of the $\beta$ coefficient. 


\subsubsection{Estimating vegetation water content using artificial neural networks}

A comparative analysis of ANN, resulting from the LMANN and ScgANN training algorithms with different network inputs, is tabulated in Table 4.3 and presents the estimated accuracy of the optimal ANN structure for each species. The reliability of ANN for estimating EWTC and $\mathrm{FMC}_{\mathrm{C}}$ is confirmed by the high values of $\mathrm{R}^{2} \mathrm{CV}$ and low values of RMSE $E_{\mathrm{CV}}$. The most remarkable result is that $\mathrm{FMC}_{\mathrm{C}}$ is retrieved with higher accuracy than $\mathrm{EWT}_{\mathrm{C}}$ for all four species. As can be seen in Table 4.3, in general, $\mathrm{FMC}_{\mathrm{C}}$ and $\mathrm{EWT}_{\mathrm{C}}$ are more accurately estimated with the LMANN than with the ScgANN training algorithm. This can be seen clearly in the estimation of both variables for Ficus benjamina, Azalea japonica, as well as the pooled data. However, for Euonymus japonicus the variables are more accurately estimated using the ScgANN algorithm; this is also the case for the EWT $_{\mathrm{C}}$ in Buxus sempervirens with all 279 narrow wavebands as network input. Of the four species, Euonymus japonicus displays the most accurate estimation of $\mathrm{FMC}_{\mathrm{C}}\left(\mathrm{R}^{2} \mathrm{CV}=\right.$ 0.89 and $\left.\mathrm{RMSE}_{\mathrm{CV}}=0.007\right)$ and $\mathrm{EWT}_{\mathrm{C}}\left(\mathrm{R}^{2} \mathrm{CV}=0.91\right.$ and $\left.\mathrm{RMSE}_{\mathrm{CV}}=0.18\right)$. As can be seen in Table 4.3, wavebands important for estimating water content at leaf level (Ullah et al. 2014) used in ANN, result in the lowest accuracy for both $\mathrm{FMC}_{\mathrm{C}}$ and $\mathrm{EWT}_{\mathrm{C}}$. The highest predicted accuracy using ANN is obtained using two and seven neurons, respectively, in hidden layers for EWT $_{C}$ and FMCC. The relationship between estimated and measured EWTC, and $\mathrm{FMC}_{\mathrm{C}}$ using ANN models is shown in Figure 4.3. Additionally, the results in Table 4.3 demonstrate that using the most important wavebands, identified with PLSR as the network input, leads to higher prediction accuracy, than using all the 279 available wavebands does among four plant species. However, as can be seen from Table 4.3, using all 279 wavebands as network input achieved a higher prediction accuracy for the pooled data for both $\mathrm{EWT}_{\mathrm{C}}$ and $\mathrm{FMC}_{\mathrm{C}}$ variables. 
Table 4.3: Cross-validated results $\left(R^{2} \mathrm{CV}\right.$ and $\left.R_{M S E}\right)$ of estimated canopy equivalent water thickness $\left(E W T_{C}\right)$ and canopy fuel moisture content $\left(F M C_{C}\right)$, obtained using artificial neural networks with different network inputs structures.

\begin{tabular}{|c|c|c|c|c|c|c|c|c|}
\hline \multirow[b]{2}{*}{ Species } & \multirow[b]{2}{*}{ Input } & \multirow{2}{*}{$\begin{array}{c}\text { Training } \\
\text { algorithm }\end{array}$} & \multicolumn{3}{|c|}{$\mathrm{FMC}_{\mathrm{C}}$} & \multicolumn{3}{|c|}{$\mathbf{E W T}_{\mathbf{C}}$} \\
\hline & & & $\begin{array}{l}\text { No. of } \\
\text { neuron }\end{array}$ & $\mathbf{R}^{2} \mathrm{CV}$ & RMSE $_{C V}$ & $\begin{array}{l}\text { No. of } \\
\text { neuron }\end{array}$ & $\mathbf{R}^{2} \mathrm{CV}$ & RMSE $_{C V}$ \\
\hline \multirow{6}{*}{ 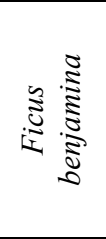 } & ANW & & 6 & 0.76 & 0.77 & 1 & 0.67 & 0.02 \\
\hline & IPW & LMANN & 6 & 0.90 & 0.49 & 5 & 0.88 & 0.01 \\
\hline & UIPW & & 7 & 0.21 & 1.38 & 2 & 0.49 & 0.03 \\
\hline & ANW & & 5 & 0.71 & 0.88 & 3 & 0.53 & 0.03 \\
\hline & IPW & ScgANN & 6 & 0.80 & 0.71 & 4 & 0.78 & 0.02 \\
\hline & UIPW & & 5 & 0.20 & 1.39 & 4 & 0.26 & 0.04 \\
\hline \multirow{6}{*}{ 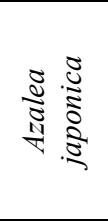 } & ANW & & 1 & 0.78 & 0.23 & 3 & 0.75 & 0.00 \\
\hline & IPW & LMANN & 1 & 0.91 & 0.13 & 2 & 0.57 & 0.01 \\
\hline & UIPW & & 3 & 0.57 & 0.28 & 7 & 0.46 & 0.01 \\
\hline & ANW & & 6 & 0.75 & 0.23 & 7 & 0.47 & 0.01 \\
\hline & IPW & ScgANN & 7 & 0.85 & 0.17 & 4 & 0.48 & 0.01 \\
\hline & UIPW & & 6 & 0.26 & 0.37 & 6 & 0.39 & 0.01 \\
\hline \multirow{6}{*}{ 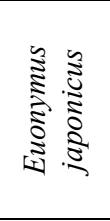 } & ANW & & 6 & 0.68 & 0.39 & 6 & 0.52 & 0.01 \\
\hline & IPW & LMANN & 7 & 0.92 & 0.19 & 2 & 0.89 & 0.00 \\
\hline & UIPW & & 6 & 0.21 & 0.67 & 6 & 0.30 & 0.02 \\
\hline & ANW & & 4 & 0.83 & 0.31 & 3 & 0.82 & 0.00 \\
\hline & IPW & ScgANN & 6 & 0.81 & 0.34 & 2 & 0.74 & 0.01 \\
\hline & UIPW & & 6 & 0.17 & 0.63 & 6 & 0.19 & 0.02 \\
\hline \multirow{6}{*}{ 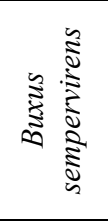 } & ANW & & 5 & 0.86 & 0.51 & 5 & 0.62 & 0.02 \\
\hline & IPW & LMANN & 3 & 0.91 & 0.41 & 5 & 0.70 & 0.02 \\
\hline & UIPW & & 7 & 0.28 & 1.57 & 3 & 0.41 & 0.03 \\
\hline & ANW & & 6 & 0.65 & 0.84 & 4 & 0.65 & 0.02 \\
\hline & IPW & ScgANN & 6 & 0.79 & 0.61 & 5 & 0.69 & 0.02 \\
\hline & UIPW & & 6 & 0.19 & 1.23 & 6 & 0.16 & 0.03 \\
\hline \multirow{6}{*}{$\begin{array}{l}0 \\
\frac{3}{3} \\
0 \\
0 \\
0 \\
0 \\
0 \\
0\end{array}$} & ANW & & 5 & 0.74 & 0.76 & 5 & 0.51 & 0.03 \\
\hline & IPW & LMANN & 5 & 0.57 & 0.90 & 7 & 0.50 & 0.03 \\
\hline & UIPW & & 7 & 0.24 & 1.17 & 5 & 0.19 & 0.03 \\
\hline & ANW & & 5 & 0.50 & 0.95 & 5 & 0.49 & 0.03 \\
\hline & IPW & ScgANN & 5 & 0.39 & 1.06 & 4 & 0.38 & 0.03 \\
\hline & UIPW & & 6 & 0.15 & 1.25 & 5 & 0.13 & 0.04 \\
\hline
\end{tabular}



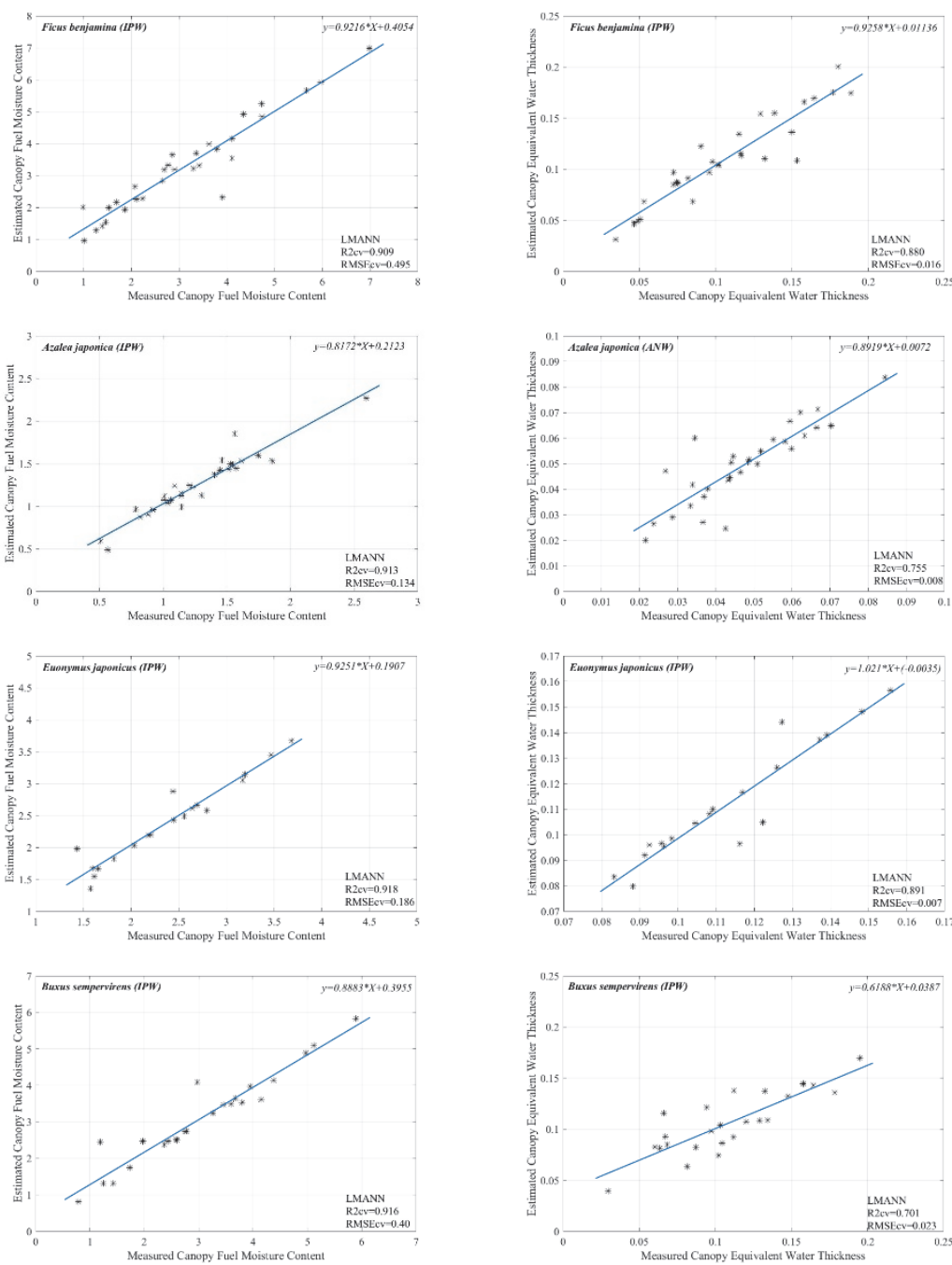

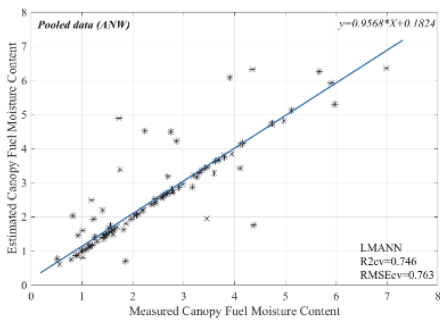

(a)

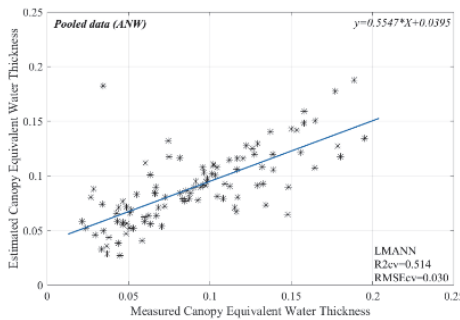

(b)

Figure 4.3: Scatterplots of measured versus estimated canopy equivalent water thickness (a), and canopy fuel moisture content (b) for the four species Ficus benjamina, Azalea japonica, Euonymus japonicus, Buxus sempervirens, and Pooled data. The blue line represents a first order (linear) regression. 


\subsection{Discussion}

This study attempts to highlight how emissivity spectra from the TIR region can estimate mass based canopy water content $\left(\mathrm{FMC}_{\mathrm{C}}\right)$ and area based canopy water content $\left(E T_{C}\right)$ with statistical methods (i.e. PLSR) and machine learning algorithms (i.e. ANN). Previous literature shows that EWTC as an area-based variable can be estimated from water absorption features in the VNIR and SWIR spectral domains (Bowyer and Danson 2004; Mirzaie et al. 2014). However, estimating the relationship between $\mathrm{FMC}_{\mathrm{C}}$ and spectral data in the optical region remains problematic (Chuvieco et al. 2003; Paltridge and Barber 1988). It seems that spectral information from these VNIR and SWIR is not adequate for retrieving $\mathrm{FMC}_{\mathrm{C}}$ due to the internal structure of leaves as well as the influence of vegetation DMC (Bowyer and Danson 2004; Ceccato et al. 2001). Our results demonstrate that both variables can be predicted reasonably well from TIR emissivity spectra. However, we demonstrate that mass-based canopy water content can be more accurately retrieved across species from hyperspectral TIR data (Tables 4.2 and 4.3) than area-based canopy water content. A possible justification for $\mathrm{FMC}_{\mathrm{C}}$ being predicted more accurately than EWT $_{C}$ using emissivity spectra may be the relation between $\mathrm{FMC}_{\mathrm{C}}$ and the combination of heat flow and mass. $\mathrm{FMC}_{\mathrm{C}}$ as a mass-based variable indirectly represents temperature and light use efficiency and also represents the water availability in the plant (Yebra et al. 2013).

In this study, $\mathrm{FMC}_{\mathrm{C}}$ and $\mathrm{EWT}_{\mathrm{C}}$ are calculated respectively by multiplying FMC and EWT with the LAI, which can be a proxy of mass. Recently Neinavaz et al. (2016b) found that there is a positive correlation between LAI and TIR emissivity spectra. As can be observed in Figure 4.1, the correlation between LAI and EWT is higher $\left(\mathrm{R}^{2}=0.25\right)$ than between LAI and FMC $\left(\mathrm{R}^{2}\right.$ $=0.05)$. Therefore, when using LAI, one may expect to retrieve EWTC with higher accuracy than FMC . However, this is not observed here. As FMC does not scale with LAI, which means that canopies with similar FMC may have varying LAI values (Figure 4.1) and, consequently, differing spectral responses (Bowyer and Danson 2004; Yebra et al. 2013). In addition, our results show that both $\mathrm{FMC}_{\mathrm{C}}$ and $\mathrm{EWT}_{\mathrm{C}}$ can be predicted with higher accuracy than LAI using TIR hyperspectral data (Neinavaz et al. 2016c).

Although our study reveals that LAI was correlated with EWT (leaf level), this relationship might not always be strictly valid under all conditions and 
may have different temporal (daily or seasonal) trends or vary across ecoregions (Trombetti et al. 2008). It should be noted that besides LAI some other factors such as canopy structure, temperature, species physiology as well as the environmental conditions can have an influence on the amount of water content in vegetation (Serrano et al. 2000; Trombetti et al. 2008). In this study, due to the stable conditions provided during the laboratory measurements, potential variation caused by environmental conditions (e.g. temperature) may be ignored. However, further experimental work is required to confirm this assertion.

As can be seen in Tables 4.2 and 4.3, our results indicate that EWTC (ANN: $\left.\mathrm{R}^{2} \mathrm{CV}=0.51, \mathrm{RMSE}_{\mathrm{CV}}=0.03\right)$ and $\mathrm{FMC}_{\mathrm{C}}\left(\mathrm{ANN}: \mathrm{R}^{2} \mathrm{CV}=0.74, \mathrm{RMSE}_{\mathrm{CV}}=\right.$ $0.76)$ have been predicted with moderate accuracy from the pooled data. However, these variables have been retrieved with higher accuracy at the species level. In other words, the observed relationships with TIR emissivity spectra using both proposed statistical techniques are likely to be speciesspecific. In this respect, the correlation between emissivity spectra in the TIR region and variation in cuticle composition in different plant species is a possible explanation (Elvidge 1988; Salisbury 1986). Recently, Buitrago et al. (2016) studied the correlation between leaf water content and TIR emissivity spectra and suggested that the plant's cuticle thickness and structure may be linked to the changes in emissivity spectra. Additionally the multi-functional properties of the cuticle, due to its heterogeneous structure and chemical nature (Khayet and Fernández 2012), may additionally vary between e.g. species, genotypes, plant physiological status as well as environmental conditions during growth (Fernández et al. 2016; Fernández et al. 2014; Guzmán-Delgado et al. 2016; Knoche et al. 2004; Szakiel et al. 2012), and could consequently affect the emissivity spectra.

Moreover, the prediction of canopy water content (in terms of EWT $\mathrm{C}$ and FMCC) in the TIR domain could be affected by differences in the vegetation canopy architecture (Pinter Jr et al. 1985) as well as canopy gap size (Ribeiro da Luz and Crowley 2010) through the control of energy transfer (Kimes 1980). Therefore, in order to obtain an accurate estimation of canopy parameters such as EWT $_{C}$ and $\mathrm{FMC}_{\mathrm{C}}$ in heterogeneous ecosystems, knowledge of other biophysical and biochemical properties of plant species is probably a prerequisite. 
Our findings revealed that compared with PLSR models, the ANN approach obtained relatively higher prediction accuracy. This may be due to the fact that ANN method as a machine learning algorithm offers a powerful means of analyzing complex datasets without making assumptions about the model parameterization. Our results are in agreement with the previous finding of Neinavaz et al. (2016) who revealed that ANN improves the prediction accuracy of canopy biophysical variables compared with PLSR using emissivity spectra over the entire TIR spectrum. Both methods (i.e. PLSR and ANN) have their strengths and limitations which have been addressed by many researchers (Cramer 1993; Mehrotra et al. 1997; Pirouz 2006).

Of the three categories of network input for ANN used to retrieve $\mathrm{EWT}_{\mathrm{C}}$ and $\mathrm{FMC}_{\mathrm{C}}$, the important wavebands identified using PLSR (i.e. IPW) were superior to the others for the prediction of these variables for individual species $\left(\mathrm{R}^{2} \mathrm{CV}=0.92, \mathrm{RMSECV}_{\mathrm{C}}=0.19\right.$ for $\mathrm{FMC}_{\mathrm{C}}$; and $\mathrm{R}^{2} \mathrm{CV}=0.89$, $\mathrm{RMSECV}_{\mathrm{C}}$ $=0.007$ for $\left.\mathrm{EWT}_{\mathrm{C}}\right)$. In contrast, the ANW network input achieved a higher prediction accuracy $\left(\mathrm{R}^{2} \mathrm{CV}=0.74, \mathrm{RMSE}_{\mathrm{CV}}=0.76\right.$ for $\mathrm{FMC}_{\mathrm{C}}$; and $\mathrm{R}^{2} \mathrm{CV}=0.51$, $\mathrm{RMSE}_{\mathrm{CV}}=0.03$ for $E W T_{\mathrm{C}}$ ) for pooled data. A possible explanation is that by incorporating important wavebands into the back-propagation neural network as input variables, over-fitting was prevented and data dimension was reduced for individual plant species. Since emissivity spectra are species-specific over TIR region (Neinavaz et al. 2016c; Ribeiro da Luz and Crowley 2007) and that spectral features in the TIR domain varied from one species to another (Fabre et al. 2011a). Therefore, the ANW input network, which contains 279 wavebands, seems more suitable to use for pooled data. Wavebands identified by Ullah et al. (2014) achieved lower accuracies estimating both variables than other network inputs (Table 4.3). The reason for this may be that their study was carried out at leaf level and that the canopy structure and architecture of different plant species did not play any role in their findings.

In this study, we did not assess the strength of the entire spectra (i.e. VNIRTIR region) for the retrieval of vegetation water content (i.e. FMC and EWT) at canopy level. However, since $\mathrm{FMC}_{\mathrm{C}}$ was predicted with higher accuracy than $\mathrm{EWT}_{\mathrm{C}}$, which is contrary to previous findings using hyperspectral and multispectral data, further experimental efforts would be required to assess the improvement in prediction accuracy of FMC and EWT at canopy level over different regions of the electromagnetic spectrum. 
The results that are reported in this study are derived from emissivity measurements under controlled laboratory conditions. Accordingly, atmospheric absorption and scattering effects have been dismissed from our data set, and possible sources of error such as atmospheric corrections, and the effect of the background have been reduced. It will require further study to quantify the possible effect of these factors on the success of estimating EWC and FMC at canopy level from TIR spectra. However, this study provides an initial basis for evaluating the potential of TIR hyperspectral remote sensing for $\mathrm{EWT}_{\mathrm{C}}$ and $\mathrm{FMC}_{\mathrm{C}}$ prediction.

\subsection{Conclusion}

Our results establish the value of thermal emissivity spectra from the TIR region for predicting $\mathrm{FMC}_{\mathrm{C}}$ and $\mathrm{EWT}_{\mathrm{C}}$ using multivariate (i.e. PLSR and ANN) statistical techniques at canopy level for multiple plant species. FMCC as a representative of mass-based variables has been retrieved more accurately than $\mathrm{EWT}_{\mathrm{C}}$ from hyperspectral TIR data. When pooled data of several plant species is considered, the prediction accuracy drops for both variables (i.e. EWT $_{\mathrm{C}}$ hand $\mathrm{FMC}_{\mathrm{C}}$ ). When combined with ANN, optimized narrow wavebands as network input data present the most satisfactory performance predicting EWTC and FMCc. Clearly, given the results of our experiments, it will be difficult, although not impossible, to extend our findings to airborne and satellite level, particularly in different ecosystems. Field conditions, including canopy structure and atmospheric conditions, coupled with the spatial and spectral resolution of satellite observations, may provide challenges when upscaling to canopy level. However, in our perspective, the findings of this study prove the concept of predicting vegetation water content at canopy level by means of hyperspectral TIR data to be valid. 


\section{Chapter 5}

\section{Successful Retrieval of Leaf Area Index from Thermal Satellite Imagery over Mixed Temperate Forest*}

* This chapter is based on:

Neinavaz, E., Darvishzadeh, R., Skidmore, A. K., \& Abdullah, H. (Under review). Successful Retrieval of Leaf Area Index from Thermal Satellite Imagery over Mixed Temperate Forest. Remote Sensing of Environment. In review. 


\begin{abstract}
Hyperspectral measurements in the thermal Infrared (TIR, 8-14 $\mu \mathrm{m})$ region have demonstrated that retrieval of leaf area index (LAI, $\mathrm{m}^{2} \mathrm{~m}^{-2}$ ) can be achieved with reasonable accuracy under controlled laboratory conditions. However, predicting LAI based on TIR data from remotely sensed images is yet to be addressed. In this study, for the first time, we quantify LAI over a mixed temperate forest, using the Landsat 8 TIR data as well as its integration with visible, near infrared, and shortwave infrared data (VNIR/SWIR 0.3-2.5 $\mu \mathrm{m})$. In August 2015, concurrent with a Landsat 8 overpass, a field campaign was conducted, and forest structural parameters including LAI and proportion of vegetation cover $(\mathrm{Pv})$ were measured at 37 field plots. Land surface emissivity (LSE) was calculated using the normalized difference vegetation index thresholds method, and the $\mathrm{Pv}$ was estimated using different approaches. We used empirical methods including six different vegetation indices as well as an artificial neural network (ANN) approach to retrieve LAI. There was a significant difference between plots regarding LSE and vegetation type. LAI was predicted with modest accuracy using TIR vegetation indices $\left(\mathrm{R}_{\mathrm{CV}}^{2}=0.35, \mathrm{RMSE}_{\mathrm{CV}}=1.20, \mathrm{~m}^{2} \mathrm{~m}^{-2}\right)$. This retrieval accuracy was improved further by using an $\mathrm{ANN}\left(\mathrm{R}^{2} \mathrm{CV}=0.55, \mathrm{RMSE}_{\mathrm{CV}}=\right.$ $\left.1.02, \mathrm{~m}^{2} \mathrm{~m}^{-2}\right)$. When the VNIR/SWIR $\left(\mathrm{R}^{2} \mathrm{CV}=0.58, \mathrm{RMSECV}_{\mathrm{C}}=0.83, \mathrm{~m}^{2} \mathrm{~m}^{-2}\right)$ and TIR data were integrated into the ANN, the estimation accuracy for the LAI was greatly increased $\left(\mathrm{R}^{2} \mathrm{CV}=0.73, \mathrm{RMSE}_{\mathrm{CV}}=0.78, \mathrm{~m}^{2} \mathrm{~m}^{-2}\right)$. Our results demonstrate that TIR satellite data can achieve reasonable accuracy for LAI retrieval using VNIR/SWIR measurements and that the combination of reflectance and emissivity data increases the retrieval accuracy of LAI. This finding has implications for the retrieval of other vegetation parameters using TIR satellite remote sensing, as well as regional mapping of LAI coupled with a canopy radiative transfer model.
\end{abstract}




\subsection{Introduction}

Leaf area index (LAI, $\mathrm{m}^{2} \mathrm{~m}^{-2}$ ) is used extensively to monitor ecosystem functions (e.g. vegetation growth, and physiological activity) (Asner et al. 2003; Barclay 1998; Gower et al. 1999). LAI is a dimensionless variable and is defined as the total of the one-sided leaf area $\left(\mathrm{m}^{2}\right)$ per unit of horizontal surface area $\left(\mathrm{m}^{2}\right)$ (Watson 1947). Due to the importance of LAI in primary production (e.g. photosynthesis), transpiration, energy exchange, and other physiological characteristics pertinent to a range of ecosystem processes, accurate prediction of LAI has been a concern in a broad spectrum of research (Bréda 2003). Moreover, LAI has recently been proposed as one of the essential biodiversity variables, among others potentially fit for satellite monitoring (Pereira et al. 2013; Skidmore et al. 2015). The demand for LAI monitoring over large areas has increased in recent years due to growing interest in monitoring and modelling of climate change and habitat degradation (Duro et al. 2007; Running et al. 2004).

Using remote sensing, in particular the visible/near-infrared (VNIR, 0.3-1.0 $\mu \mathrm{m}$ ) and shortwave infrared (SWIR, 1.0-2.5 $\mu \mathrm{m}$ ) spectral data, vegetation biophysical variables such as LAI have been widely retrieved in different ecosystems and with a varying degree of success (Bacour et al. 2006; Baret and Guyot 1991; Baret et al. 1989; Boegh et al. 2002; Broge and Mortensen 2002; Brown et al. 2000; Darvishzadeh et al. 2008b; Zheng and Moskal 2009). The potential of thermal infrared (TIR) remote sensing for estimating vegetation biophysical variables in general, and LAI in particular, has been the subject of very limited research. The advantage of using TIR data for remote sensing of vegetation is that homo-nuclear diatomic molecules such as $\mathrm{N}_{2}$ and $\mathrm{O}_{2}$ do not exhibit strong spectral features in the wavelengths occurring between $8 \mu \mathrm{m}$ and $14 \mu \mathrm{m}$; consequently, this atmospheric window is largely transparent (Clerbaux et al. 2011). Recently, LAI has been successfully quantified using emissivity spectra in the thermal infrared (TIR, 8-14 $\mu \mathrm{m}$ ) domain under controlled laboratory conditions (Neinavaz et al. 2016c). However, the applicability and upscalability of laboratory studies on LAI estimation to landscape level remain to be proven. In this study, we utilized land surface emissivity (LSE) calculated from Landsat 8 TIR data of the Bavarian Forest National Park (BFNP) to retrieve LAI. LSE is a measure of the inherent efficiency of the surface in converting kinetic into radiant energy above the surface (Sobrino et al. 2001). Knowledge about the LSE is 
necessary for estimating the energy budget, the evapotranspiration rate, as well as the water and energy balance (Jiménez-Muñoz et al. 2006; Seemann et al. 2008; Sobrino et al. 2002). LSE is also a critical parameter for retrieving land surface temperature (Dash et al. 2002), especially when only one thermal band is available (Gillespie et al. 1998; Sobrino et al. 2004).

Several approaches exist to estimate LSE using remotely sensed data (Sobrino et al. 2008). Among these approaches, the normalized difference vegetation index threshold method (NDVI ${ }^{\mathrm{THM}}$ ) (Van de Griend and Owe 1993), which has been further modified and developed by Sobrino and Raissouni (2000), has attracted special attention as a practical approach (Sobrino et al. 2001). To calculate the LSE using the NDVI ${ }^{\mathrm{THM}}$, prior knowledge of the emissivity of vegetation and bare soil, as well as estimates of the proportion of vegetation cover $\left(\mathrm{P}_{\mathrm{v}}\right)$, are necessary. Employing vegetation indices such as the normalized difference vegetation index (NDVI) is one of the traditional approaches to estimating $\mathrm{P}$. However, NDVI becomes saturated at high vegetation cover (Atzberger 2004; Baret and Guyot 1991; Danson and Plummer 1995; Gitelson et al. 2002), which may influence the accuracy of the estimated $\mathrm{Pv}$. Consequently, this study first investigates the estimation of $\mathrm{Pv}$ for the calculation of LSE and further examines the retrieval of LAI in a mixed temperate forest using the derived LSE.

Previously, Breunig et al. (2009) revealed that the combined use of SWIR (i.e., reflectance) and TIR (i.e., emissivity) data enhanced discrimination between nonphotosynthetic vegetation and exposed soil. Also, a recent study by Mushore et al. (2016) showed that the integration of Landsat 8 data from the operational land imager (OLI, 0.4-2.5 $\mu \mathrm{m}$ ) and the thermal infrared sensor (TIRS, 10.6-12.5 $\mu \mathrm{m}$ ) achieved significantly higher accuracy in classifying urban landscapes. However, to our knowledge, the combined use of reflectance and emissivity data has not been investigated in vegetation studies for predicting LAI. The first section of this paper is dedicated to deriving the LSE using Pv calculated from an artificial neural network (ANN) as well as through empirical approaches. The derived LSE values are then used to estimate LAI by means of univariate (vegetation indices) and multivariate (ANN) techniques. Finally, the reflectance and emissivity data from the OLI and TIRS bands of Landsat 8 are integrated to examine the LAI retrieval accuracy. 


\subsection{Materials and methods}

\subsubsection{General description of the study area}

Field measurements were carried out over the BFNP located in south-eastern Germany along the border with the Czech Republic (49 $3^{\circ} 19^{\prime \prime} \mathrm{N}, 13^{\circ} 12^{\prime} 9^{\prime \prime}$ E) (Figure 5.1). The BFNP has a total area of 24,250 hectares (Huber 2005). Elevation in this area ranges from $600 \mathrm{~m}$ to $1453 \mathrm{~m}$ above sea level. The climate of the region is temperate. Precipitation varies from 1200 to 1800 $\mathrm{mm} /$ year (of which $50 \%$ is snow), and in some years exceeds $2000 \mathrm{~mm}$ (Huber 2005). The minimum average annual temperature is between $3^{\circ} \mathrm{C}$ and $6^{\circ} \mathrm{C}$. Three major forest types are recognizable in the BFNP: above $1100 \mathrm{~m}$, at high altitude, there are sub-alpine spruce forests with Norway spruce (Picea abies) and some Mountain ash (Sorbus aucuparia); on the slopes, between $600 \mathrm{~m}$ and $1100 \mathrm{~m}$, mountain mixed forests with Norway spruce, Silver fir (Abies alba), European beech (Fagus sylvatica), and Norway maple (Acer pseudoplatanus) can be found; in wet depressions in the lower valley (i.e. valley bottoms), Spruce forests mixed with Norway spruce, Mountain ash, and Birches (Betula pendula, and Betula pubescens) occur (Heurich 2008; Reitberger et al. 2006). In general, the dominant tree species in the BFNP are Norway spruce (67\%), European beech (24.5\%), and Fir (2.6\%) (Heurich et al. 2010). 


\section{Bavarian Forest National Park}
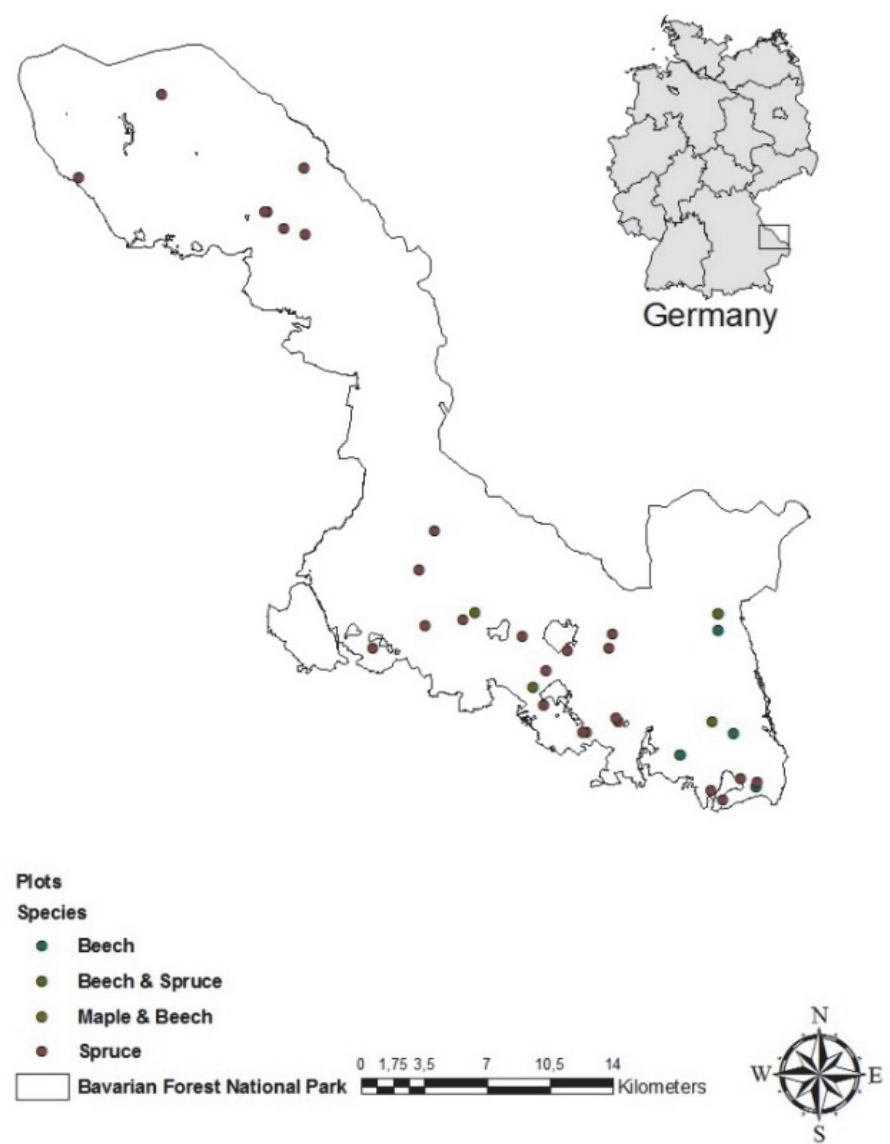

Figure 5.1: Location of the Bavarian Forest National Park, Germany, and the location of the sample plots.

\subsubsection{In situ, structural canopy parameter collection}

A field campaign was conducted in August 2015. The study area was stratified into broadleaf, needle leaf, and mixed forest stands. The stratified random sampling strategy was adopted, and 37 plots of $30 \times 30 \mathrm{~m}$ were selected resulting in 4 broad-leaf, 26 needle-leaf, and 7 mixed forest plots. The coordinates of the center location of the plots were recorded by a Leica system GPS 1200 with an accuracy of approximately $1 \mathrm{~m}$ after post processing (Leica Geosystems AG, Heerbrugg, Switzerland). At each plot, species types were recorded, and structural forest parameters including LAI and $\mathrm{P}_{\mathrm{V}}$ were measured. LAI was measured using the plant canopy analyzer LAI-2200 
(LICOR Inc., Lincoln, NE, USA). For each plot, reference samples of the above-canopy radiation were collected by measuring incoming radiation in a nearby open area. Next, five below-canopy samples were measured within the plot. The LAI value of each plot was then calculated by averaging these measurements. $\mathrm{Pv}$ or fractional vegetation cover initially was introduced by Deardorff (1978) and defined as the ratio of the vertical projection area of vegetation (including leaves, stalks, and branches) on the ground to the total vegetation area (Salimi Kouchi et al. 2013). The $\mathrm{P}_{\mathrm{v}}$ of each plot was computed using five upward-pointing hemispherical photographs. A digital hemispherical camera was used to measure $\mathrm{P}_{\mathrm{V}}$ according to the proposed guidelines by Zhou et al. (1998). The images were acquired using a Canon 5D equipped with a fisheye lens leveled on a tripod at approximately breast height (1.3 $\mathrm{m}$ above the ground) (Whitmore et al. 1993). The arithmetic mean of the $\mathrm{P}_{\mathrm{v}}$ calculated from the images was then considered as $\mathrm{P}_{\mathrm{v}}$ of the plot.

\subsubsection{Satellite data and processing}

The satellite data were acquired on 9 August 2015 from Landsat 8 for the study area. The Landsat 8 satellite has two main sensors, the OLI and the TIRS (Table 5.1). Since Landsat images are not atmospherically corrected, the OLI and TIRS images were corrected by converting digital numbers to radiance values, using the coefficients supplied by the USGS (United States Geological Survey) (https://landsat.usgs.gov/using-usgs-landsat-8-product). For the OLI images, radiance was converted to reflectance using the Fast Line-of-sight Atmospheric Analysis of Spectral Hypercubes (FLAASH) module. FLAASH is an MODTRAN4-based atmospheric correction software package developed by the Air Force Phillips Laboratory, Hanscom AFB, and Spectral Sciences, Inc. (Adler-Golden et al. 1999) and is available in the Research Systems Inc. ENVI software package. In this study, NDVI ${ }^{\mathrm{THM}}$ was applied to estimate LSE. Therefore, the atmospheric correction was not needed for the TIRS bands ( $\mathrm{Li}$ et al. 2013). Bands 10 and 11 in the TIR region were acquired at $100 \mathrm{~m}$ resolution and were resampled to $30 \mathrm{~m}$ to match with the OLI spectral bands. 
Table 5.1: The Landsat 8 sensors, the operational land imager (OLI) and the thermal infrared sensor (TIRS) spectral bands and spatial resolution.

\begin{tabular}{cccc}
\hline Landsat 8 Sensor & Bands & Wavelength $(\boldsymbol{\mu m})$ & Resolution (m) \\
\hline \multirow{4}{*}{ OLI } & Band 1 & $0.43-0.45$ & 30 \\
& Band 2 & $0.45-0.51$ & 30 \\
& Band 3 & $0.53-0.59$ & 30 \\
& Band 4 & $0.64-0.67$ & 30 \\
& Band 5 & $0.85-0.88$ & 30 \\
& Band 6 & $1.57-1.65$ & 30 \\
& Band 7 & $2.11-2.29$ & 30 \\
\hline \multirow{2}{*}{ TIRS } & Band 10 & $10.60-11.19$ & 100 \\
& Band 11 & $11.50-12.51$ & 100 \\
\hline
\end{tabular}

\subsubsection{Estimation of Land Surface Emissivity}

One of the practical methods to determine LSE is using statistical relationships between the logarithms of NDVI and surface emissivity in thermal infrared bands (Van de Griend and Owe 1993). This approach was later modified and called NDVI ${ }^{\mathrm{THM}}$ (Sobrino and Raissouni 2000; Valor and Caselles 1996). Emissivity based on the $\mathrm{NDVI}^{\mathrm{THM}}$ is calculated as follows (Sobrino and Raissouni 2000; Sobrino et al. 2008):

$\varepsilon_{\lambda}=\left\{\begin{array}{lc}\text { for NDVI }<0.2, & \alpha_{\lambda}+b_{\lambda} \rho_{\text {red }} \\ \text { for NDVI } \geq 0.5, & \varepsilon_{\mathrm{v} \lambda}+\mathrm{d}_{\varepsilon} \\ \text { for } 0.2 \leq \mathrm{NDVI} \leq 0.5, & \varepsilon_{\mathrm{v} \lambda} \mathrm{P}_{\mathrm{v}}+\varepsilon_{\mathrm{s} \lambda} \times\left(1-\mathrm{P}_{\mathrm{v}}\right)+\mathrm{d}_{\varepsilon}\end{array}\right.$

where $\alpha$ and $b$ are the soil line coefficients at wavelength $\lambda, \rho_{\text {red }}$ denotes the reflectivity values in the red region, $\mathrm{d} \varepsilon$ is the cavity effect, $\varepsilon_{v \lambda}$ and $\varepsilon_{s \lambda}$ are band emissivity values for vegetation and bare soil, respectively, calculated from the MODIS UCSB (University of California, Santa Barbara) emissivity library (Wan and Dozier 1996), and $\mathrm{P}_{\mathrm{V}}$ denotes the proportion of vegetation cover. For plain surfaces, $d \varepsilon$ is negligible, but for heterogeneous and rough surfaces such as forest, this term can reach a value of 2\% (Sobrino 1989; Sobrino et al. 1990). A good approximation for this term can be given by the following equation:

$d_{\varepsilon}=\left(1-\varepsilon_{s}\right)\left(1-P_{v}\right) F \varepsilon_{v}$

where $F$ denotes a shape factor with a mean value, assuming different geometrical distributions, of 0.55 (Sobrino et al. 1990; Sobrino et al. 2004). 
Taking into account Eq. (1.a-c), the land surface emissivity can be attained through:

$\varepsilon=m P_{v}+n$

with

$m=\varepsilon_{v \lambda}-\varepsilon_{s \lambda}-\left(1-\varepsilon_{s \lambda}\right) F \varepsilon_{v \lambda}$

$n=\varepsilon_{s \lambda}+\left(1-\varepsilon_{s \lambda}\right) F \varepsilon_{v \lambda}$

\subsubsection{Estimating the proportion of vegetation}

As can be observed from Eq. (3), $\mathrm{P}_{\mathrm{V}}$ is an input parameter for calculating LSE. Several methods exist in the literature to estimate $P_{v}$ using empirical models. For instance, Gutman and Ignatov (1998) estimated Pv using the NDVI (Rouse et al. 1974). However, NDVI becomes saturated in high vegetation cover (Atzberger 2004; Baret and Guyot 1991; Danson and Plummer 1995; Gitelson et al. 2002). To resolve this issue, Gitelson et al. (2002) have suggested a variable atmospherically resistant index (VARIgreen), substituting near infrared reflectance with green reflectance, and also using the concept of the atmospherically resistant vegetation index (Kaufman and Tanre 1992) to compensate for atmospheric effects. Based on this concept, Jiménez-Muñoz et al. (2009) estimated the PV of agricultural land (corn, alfalfa, etc.) using the VARIgreen. An alternative approach to using spectral indices for the estimation of biophysical variables such as $\mathrm{P}_{\mathrm{V}}$ is to apply fieldbased measurements and spectral reflectance to train an ANN. Boyd et al. (2002) suggested the ANN approach be the premier choice for predicting Pv. Therefore, in this study, surface reflectance values of bands of the OLI sensor in the $0.4 \mu \mathrm{m}$ to $2.5 \mu \mathrm{m}$ spectral domain were used to estimate the $P_{v}$ by (i) calculating the VARIgreen and (ii) employing the ANN approach (hereafter, referred to as $\mathrm{PV}_{\mathrm{V}}{ }^{\text {VARIgreen }}$ and $\mathrm{PV}^{\mathrm{ANN}}$, respectively). For network training, the Levenberg-Marquardt (LMANN) algorithm was utilized, as it is one of the common training algorithms in back-propagation networks. Although there are no principles that determine the optimal number of hidden layers, Skidmore et al. (1997) noted that higher accuracy would be obtained with three hidden layers, than with two or one. As the prediction performance of an ANN depends on the number of neurons in the hidden layer, the optimal ANN size was identified by testing different numbers. To avoid over-fitting, the early stopping technique was used. In this technique, training stops as soon 
as performance on a validation data set begins to worsen (Nowlan and Hinton 1992). Linear regression analyses were performed between the predicted $\mathrm{P}_{\mathrm{V}}$ (i.e. the network outputs) and the measured $\mathrm{P}_{\mathrm{v}}$, to identify the best ANN model. To reduce undesired effects from the random initialization of the optimization routine, the cross-validation procedure was repeated 1000 times, and results were averaged. The reliability of the ANN in estimating Pv was evaluated using the cross-validated coefficient of determination $\mathrm{R}^{2} \mathrm{Cv}$ and the cross-validated Root Mean Squared Error RMSEcv. The estimated $\mathrm{P}_{\mathrm{v}}$ was then used to calculate the LSE according to Eq. (3). The correlation between measured LAI and LSE was quantified using Pearson's r correlation. A oneway ANOVA was applied to explore whether the mean LSE significantly differs between plots with dissimilar vegetation types. Tukey's honestly significant difference test was used as a posthoc test, to determine the statistical significance of these differences.

\subsubsection{Estimation of leaf area index}

\subsubsection{Estimation of leaf area index using vegetation indices}

The most common indices for retrieving LAI from optical remote sensing data are ratio based indices. Six different vegetation indices, widely applied in the literature to derive LAI, were examined in this study. The indices were systematically calculated from the LSE derived from thermal bands. The coefficient of determination $\left(\mathrm{R}^{2}\right)$ of band combinations for each index and LAI were used to assess the strength of the relation between proposed indices and LAI. The indices that achieved higher accuracies were noted. The naming conventions, acronyms, and calculations for the indices are listed in Table 5.2. 
Table 5.2: Vegetation indices used in this study for the retrieval of leaf area index.

\begin{tabular}{|c|c|c|c|c|}
\hline Spectral Index & Original Equation & Equation & Abbreviation & Reference \\
\hline $\begin{array}{c}\text { Ratio vegetation } \\
\text { index }\end{array}$ & $* \frac{\rho_{N I R}}{\rho_{\text {Red }}}$ & $\frac{\mathcal{E}_{\lambda 1}}{\mathcal{E}_{\lambda 2}}$ & SR & (Jordan 1969) \\
\hline $\begin{array}{c}\text { Modified Simple } \\
\text { Ratio }\end{array}$ & $\frac{\rho_{\text {NIR }}-\rho_{\text {Red }}-1}{\left(\rho_{\text {NIR }} / \rho_{\text {Red }}\right)^{0.5}+1}$ & $\frac{\varepsilon_{\lambda 1}-\varepsilon_{\lambda 2}-1}{\left(\varepsilon_{\lambda 1} / \mathcal{E}_{\lambda 2}\right)^{0.5}+1}$ & MSR & (Chen 1996) \\
\hline $\begin{array}{c}\text { Difference } \\
\text { Vegetation Index }\end{array}$ & $\rho_{R e d}-\rho_{N I R}$ & $\varepsilon_{\lambda 1}-\varepsilon_{\lambda 2}$ & $\mathrm{SD}$ & $\begin{array}{c}\text { (Tucker } \\
1979)\end{array}$ \\
\hline $\begin{array}{c}\text { Normalized } \\
\text { difference } \\
\text { vegetation index }\end{array}$ & $\frac{\rho_{\text {NIR }}-\rho_{\text {Red }}}{\rho_{\text {NIR }}+\rho_{\text {Red }}}$ & $\frac{\varepsilon_{\lambda 1}-\varepsilon_{\lambda 2}}{\varepsilon_{\lambda 1}+\varepsilon_{\lambda 2}}$ & NDVI & $\begin{array}{c}\text { (Rouse et al. } \\
1974 \text { ) }\end{array}$ \\
\hline $\begin{array}{c}\text { Renormalized } \\
\text { Difference Index }\end{array}$ & $\frac{\rho_{\text {NIR }}-\rho_{\text {Red }}}{\sqrt{\rho_{N I R}+\rho_{\text {Red }}}}$ & $\frac{\mathcal{E}_{\lambda 1}-\varepsilon_{\lambda 2}}{\sqrt{\varepsilon_{\lambda 1}+\varepsilon_{\lambda 2}}}$ & RDI & $\begin{array}{c}\text { (Roujean and } \\
\text { Breon 1995) }\end{array}$ \\
\hline $\begin{array}{c}\text { Modified } \\
\text { Vegetation Index }\end{array}$ & $\frac{\rho_{\text {NIR }}-1.2 \rho_{\text {Red }}}{\rho_{\text {NIR }}+\rho_{\text {Red }}}$ & $\frac{\mathcal{E}_{\lambda 1}-1.2 \varepsilon_{\lambda 2}}{\varepsilon_{\lambda 1}+\varepsilon_{\lambda 2}}$ & MVI & $\begin{array}{c}\text { (Paltridge and } \\
\text { Barber 1988) }\end{array}$ \\
\hline
\end{tabular}

\subsubsection{Estimation of leaf area index using artificial neural networks}

Recently, LAI was successfully retrieved using TIR hyperspectral data and an ANN as a multivariate technique under controlled laboratory conditions (Neinavaz et al. 2016c). Three scenarios, based on a combination of surface reflectance (VNIR/SWIR region, bands 1 to 7/ Landsat 8 - OLI), LSE (TIR region, bands 10 and 11/Landsat 8-TIR) and integration of all bands, were applied in this study as input layers to estimate LAI using an ANN (Table 5.3). Linear regression analyses were performed between the predicted LAI and the measured LAI. For network training, the Levenberg-Marquardt (LMANN) algorithm was used. The reliability of the ANN in estimating LAI was evaluated using $\mathrm{R}^{2} \mathrm{Cv}$ and $\mathrm{RMSE} \mathrm{CV}_{\mathrm{C}}$. All analyses were executed using the MATLAB neural network toolbox. 
Table 5.3: Different input networks for estimation of LAI using artificial neural networks. LSE represents land surface emissivity and was calculated using two thermal bands in the thermal infrared region; OLI bands represent reflectance spectra from seven bands in the visible-near infrared and short-wave infrared regions.

\begin{tabular}{ccccc}
\hline No. & OLI bands & TIRS bands (LSE) & Input & Output \\
\hline 1 & $\checkmark$ & - & 7 bands & 1 \\
2 & $\checkmark$ & $\checkmark$ & 9 bands & 1 \\
3 & - & $\checkmark$ & 2 bands & 1 \\
\hline
\end{tabular}

\subsection{Results}

\subsubsection{Leaf area index and proportion of vegetation}

Summary statistics of the measured LAI and $\mathrm{P}_{\mathrm{V}}$ are tabulated in Table 5.4. The measured LAI from the 37 sample plots revealed a high maximum value $\left(\max =5.86, \mathrm{~m}^{2} \mathrm{~m}^{-2}\right)$. The measured $\mathrm{P} v$ ranged from 0.39 to 0.82 (range $=$ 0.43 ) and showed a maximum value of 0.82 . The relationship between the field measured LAI and $\mathrm{P}_{\mathrm{V}}$ is presented in Figure 5.2.

Table 5.4: Summary statistics of the measured leaf area index (LAI, $\mathrm{m}^{2} \mathrm{~m}^{-2}$ ) and proportion of vegetation cover $\left(\mathrm{P}_{\mathrm{V}}\right)$ in Bavarian Forest National Park $(n=37)$.

\begin{tabular}{|c|c|c|c|c|c|c|}
\hline \multirow{2}{*}{ Variables } & \multirow{2}{*}{ Minimum } & \multirow{2}{*}{ Maximum } & \multicolumn{2}{|c|}{ Mean } & \multirow{2}{*}{$\begin{array}{c}\text { Std. } \\
\text { Deviation }\end{array}$} & \multirow{2}{*}{$\begin{array}{l}\text { Coefficient } \\
\text { of variation }\end{array}$} \\
\hline & & & Statistic & Std. Error & & \\
\hline LAI & 0.50 & 5.86 & 3.34 & 0.24 & 1.46 & 43.60 \\
\hline $\mathrm{P}_{\mathrm{V}}$ & 0.39 & 0.82 & 0.61 & 0.02 & 0.12 & 20.51 \\
\hline
\end{tabular}

\subsubsection{Estimation of proportion of vegetation cover}

$\mathrm{P}_{\mathrm{V}}$ calculated using the ANN approach exhibited a wide range $(\max =0.82$, $\min =0.39$, range $=0.43$, mean $=0.61)$ compared with VARIgreen $(\max =$ $0.39, \min =0.29$, range $=0.10$, mean $=0.36)($ Figure 5.3). The relationship between estimated $\mathrm{P}_{\mathrm{V}}{ }^{\mathrm{ANN}}, \mathrm{P}_{\mathrm{V}}{ }^{\text {VARIgreen }}$ and in situ measurements was also investigated and is presented in Figure 5.4. As can be seen in Figure 5.4, the $\mathrm{P}_{\mathrm{V}}$ is estimated with reasonable accuracy using the ANN method $\left(\mathrm{R}^{2}=0.64\right.$, RMSE $=0.05)$ when compared to using the VARIgreen $\left(\mathrm{R}^{2}=0.42, \mathrm{RMSE}=\right.$ 0.06). Therefore, further analysis has been performed with the $\mathrm{Pv}_{\mathrm{v}}$ being calculated using the ANN. Hereafter, the LSE, which has been calculated based on the estimated $\mathrm{PV}_{\mathrm{V}}$ using the ANN approach, will be referred to as LSEPV $^{\text {ANN }}$. 


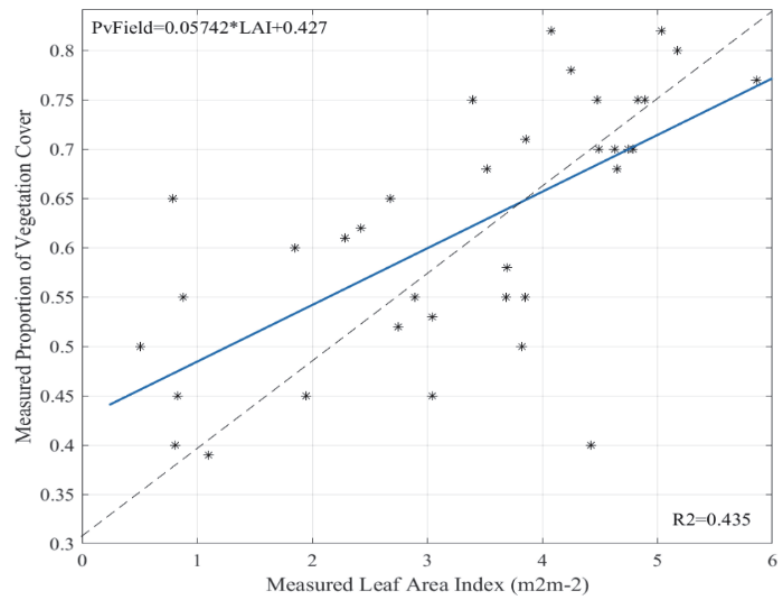

Figure 5.2: The relationship between the measured leaf area index and proportion of vegetation cover. The blue line represents a first order (linear) regression.

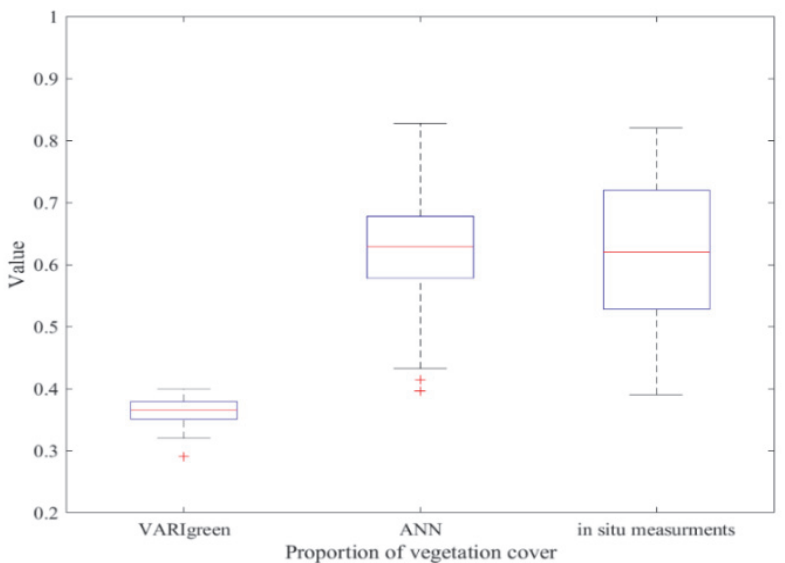

Figure 5.3: Boxplots are demonstrating the median, lower and upper quartile values of the proportion of vegetation cover calculated using artificial neural networks $(\mathrm{ANN})$, variable atmospherically resistant index values (VARIgreen) and in situ measurements. 


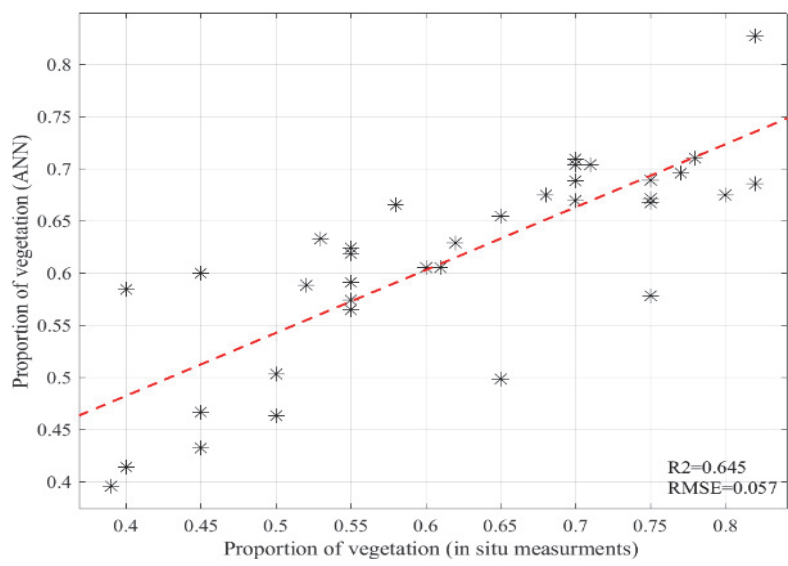

(a)

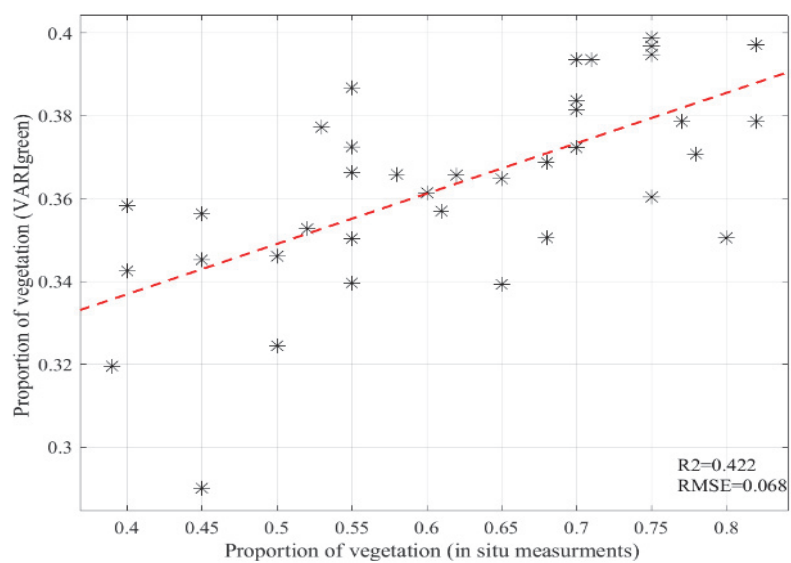

(b)

Figure 5.4: Scatterplots of measured versus predicted proportion of vegetation cover calculated from artificial neural networks (ANN) (a), and variable atmospherically resistant index values (VARIgreen) (b).

\subsubsection{Retrieval of land surface emissivity}

Applying the procedure defined for the estimation of LSE in the previous section to Landsat 8 data (i.e section 5.2.4), the LSE of the BFNP for TIRS band $10\left(\mathrm{LSE}=0.0009035 \times \mathrm{P}_{\mathrm{V}}+0.9852\right)$, and band $11\left(\mathrm{LSE}=0.0009035 \times \mathrm{P}_{\mathrm{V}}\right.$ +0.9931 ) were obtained. The LSE histograms of sample plots (i.e. LSEPV ${ }^{\mathrm{ANN}}$ ) and their boxplots are shown in Figure 5.5. 

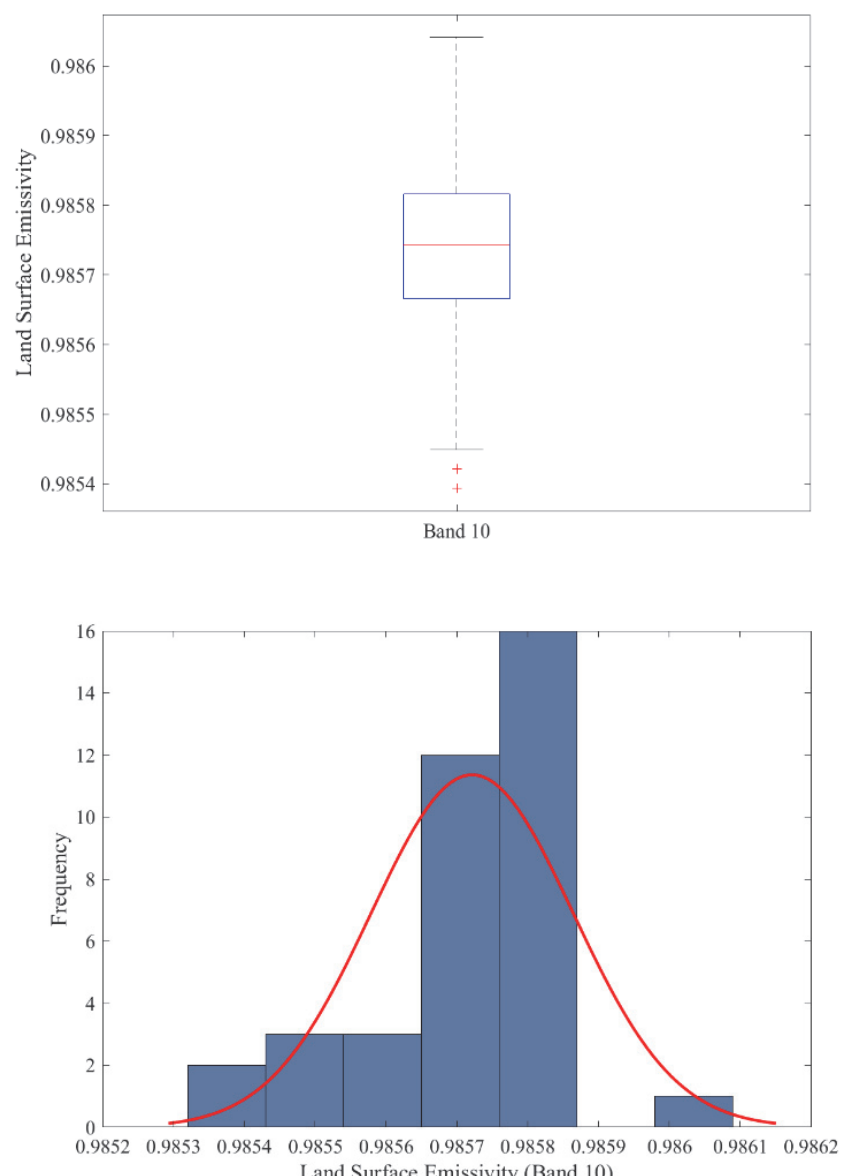
Land Surface Emissivity (Band 10)

(a) 

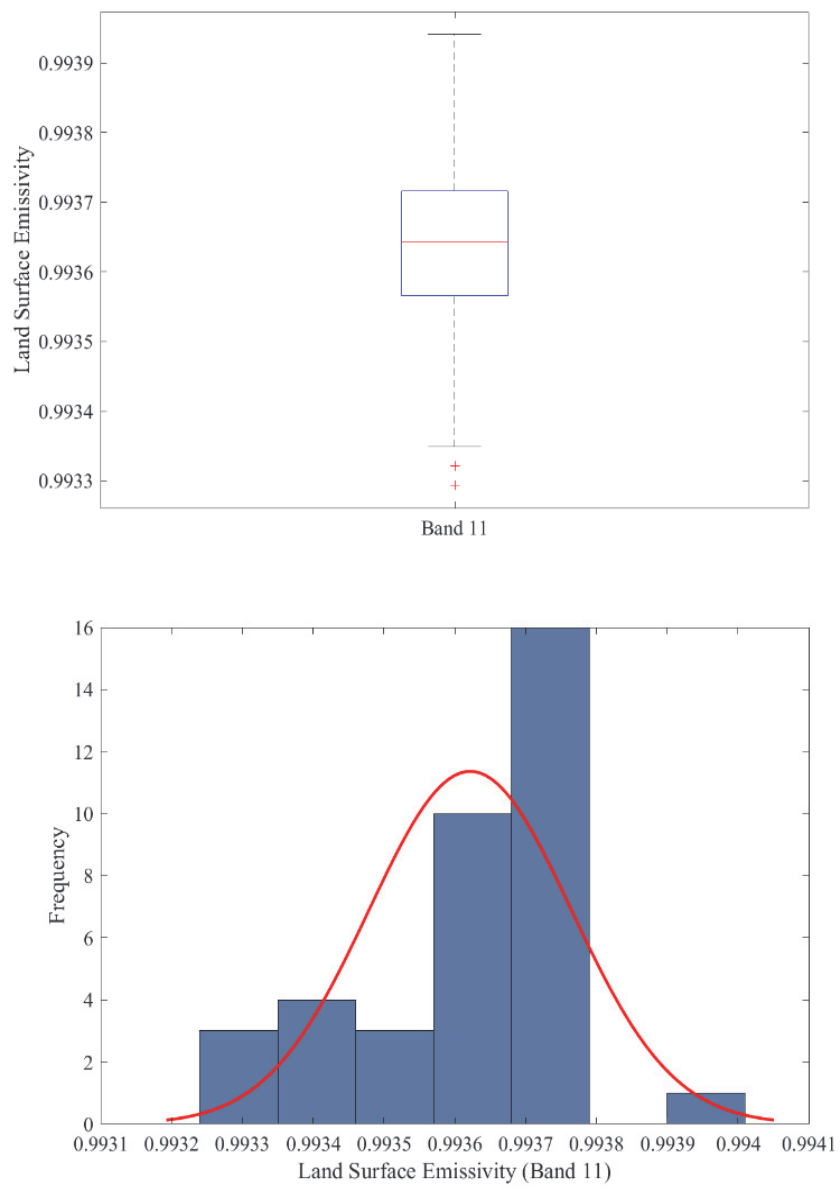

(b)

Figure 5.5: Boxplots and histograms represent the normal distribution of extracted land surface emissivity of 37 plots from TIRS band 10 (a), and band 11 (b), Landsat 8 in the Bavarian Forest National Park.

The one-way ANOVA showed that there is a significant difference ( $p$-value $<0.05$ ) between the LSE of plots with different vegetation types (Table 5.5). However, this relationship was not significant between Beech and mixed (including Beech and Spruce) plots. This was further confirmed when we plotted the LSE of different plots with the same LAI value. As can be observed from Figure 5.6, the difference in LSE is more pronounced between Spruce plots and Beech plots than between mixed plots and Beech plots. 
Table 5.5: Summary of statistics regarding One-way ANOVA with Post-hoc Tukey's HSD test of land surface emissivity (LSE) and vegetation type $(n=37)$. The mean difference is significant at the $\mathbf{0 . 0 5}$ level.

\begin{tabular}{|c|c|c|c|c|c|c|}
\hline \multirow[b]{2}{*}{$\begin{array}{l}\text { Dependent } \\
\text { Variable }\end{array}$} & \multirow[b]{2}{*}{ (I) Variable } & \multirow[b]{2}{*}{ (J) Variable } & \multirow[b]{2}{*}{ Std. Error } & \multirow[b]{2}{*}{ Sig. } & \multicolumn{2}{|c|}{ 95\% Confidence Interval } \\
\hline & & & & & $\begin{array}{l}\text { Lower } \\
\text { Bound }\end{array}$ & $\begin{array}{l}\text { Upper } \\
\text { Bound }\end{array}$ \\
\hline \multirow{6}{*}{$\mathrm{LSEPV}^{\mathrm{ANN}}$} & \multirow{2}{*}{ Beech } & $\begin{array}{l}\text { Beech \& } \\
\text { Spruce }\end{array}$ & 0.0000708 & 0.765 & -0.000223 & 0.000124 \\
\hline & & Spruce & 0.0000607 & $0.002 *$ & -0.000375 & -0.000078 \\
\hline & Beech \& & Beech & 0.0000708 & 0.765 & -0.000124 & 0.000223 \\
\hline & Spruce & Spruce & 0.0000481 & $0.002 *$ & -0.000295 & -0.000059 \\
\hline & \multirow[b]{2}{*}{ Spruce } & Beech & 0.0000607 & $0.002 *$ & 0.000078 & 0.000375 \\
\hline & & $\begin{array}{l}\text { Beech \& } \\
\text { Spruce }\end{array}$ & 0.0000481 & $0.002 *$ & 0.000059 & 0.000295 \\
\hline
\end{tabular}

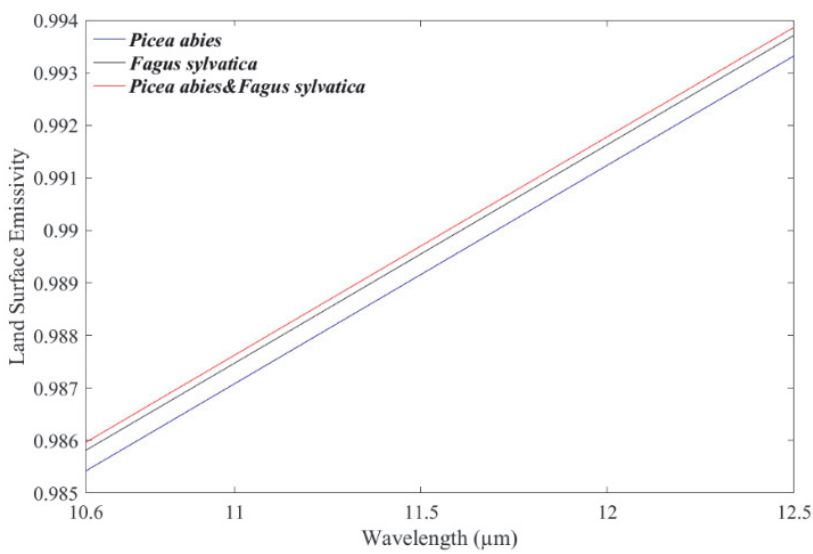

Figure 5.6: The land surface emissivity of plots with different vegetation types and an approximate LAI value of $4 \mathrm{~m}^{2} \mathrm{~m}^{-2}$.

\subsubsection{Relationships between leaf area index and land surface emissivity}

The relationship between LSE, obtained for each plot, and LAI was quantified. A positive correlation existed between LSE and LAI $(\mathrm{r}=0.66 ; p$ value $<0.001)$. The robustness of this relationship was tested using a first order polynomial and is presented in Figure 5.7. From the scatter plots in Figure 5.7, a linear relation between LAI and LSE is evident. The regression parameters are negligible with a slope of 0.00 and an offset of $0.98 ; \mathrm{R}^{2}$ is 0.44 . 


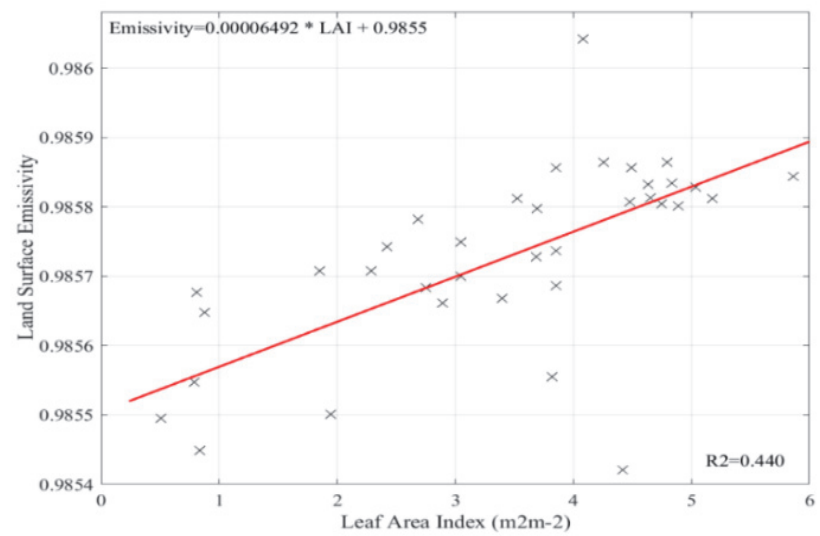

Figure 5.7: Scatterplots of in situ measured leaf area index (LAI) and land surface emissivity for 37 plots for Landsat 8 thermal bands.

\subsubsection{Estimation of leaf area index using vegetation indices in the thermal infrared region}

LAI was predicted with moderate accuracy using considered vegetation indices, although a comparison of $\mathrm{R}^{2} \mathrm{CV}$ and $\mathrm{RMSECV}$ values among investigated indices revealed no significant differences (Table 5.6). The highest $\mathrm{R}^{2}$ value obtained between LAI and LSE was $0.44\left(\mathrm{RMSE}_{\mathrm{CV}}=1.20\right.$, $\mathrm{m}^{2} \mathrm{~m}^{-2}$ ). The scatter plots in Figure 5.8, demonstrated that there is a linear relationship between estimated and measured LAI. Additionally, our findings suggest that vegetation indices calculated from LSE tend to overestimate the LAI values of below $1\left(\mathrm{~m}^{2} \mathrm{~m}^{-2}\right)$ while performing more accurately for LAI values between 2 and $5\left(\mathrm{~m}^{2} \mathrm{~m}^{-2}\right)$.

Table 5.6: The coefficients of determination $\left(R^{2}\right)$ for different indices calculated using land surface emissivity (LSE) and leaf area index. $R M S E_{C V}$ and $R^{2} \mathrm{Cv}$ are the cross-validated root mean square error and coefficient of determination between measured and estimated LAI, respectively.

\begin{tabular}{ccccc}
\hline \multirow{2}{*}{ LSE } & \multirow{2}{*}{ Index } & \multirow{2}{*}{$\mathbf{R}^{\mathbf{2}}$} & \multicolumn{2}{c}{ Validation } \\
\cline { 4 - 5 } & & $\mathbf{R}^{2} \mathbf{C V}$ & $\mathbf{R M S E}_{\mathbf{C V}}$ \\
\hline & SD & 0.44 & 0.35 & 1.20 \\
& SR & 0.44 & 0.35 & 1.20 \\
LSE $_{\mathrm{PV}}{ }^{\mathrm{ANN}}$ & NDI & 0.44 & 0.35 & 1.20 \\
& RDI & 0.44 & 0.35 & 1.20 \\
& MSR & 0.44 & 0.35 & 1.20 \\
& MVI & 0.44 & 0.35 & 1.20 \\
\hline
\end{tabular}




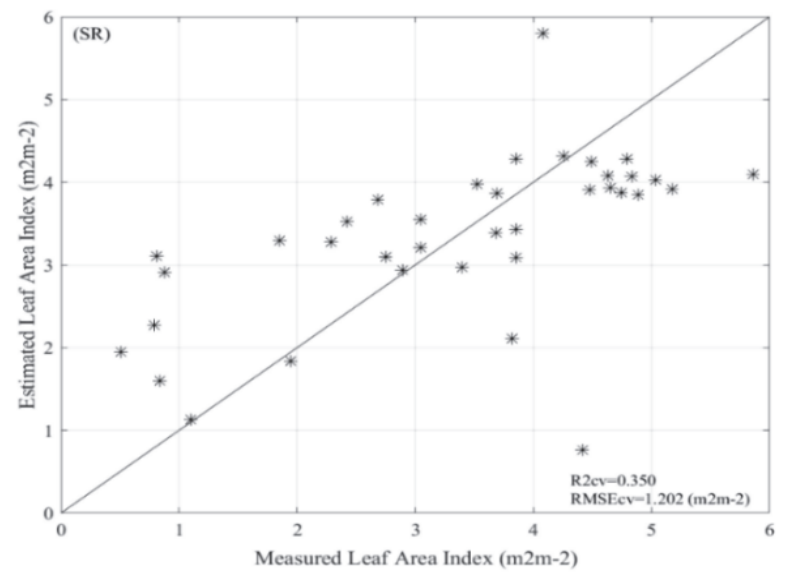

Figure 5.8: Scatterplot of measured versus predicted leaf area index calculated from the simple ratio index (SR).

\subsubsection{Estimating leaf area index using artificial neural networks}

Next, LAI was retrieved with a combination of spectral data from the VNIR/SWIR and TIR using ANN. Comparing the three scenarios tabulated in Table 5.3, the combination of spectral information from OLI and TIRS bands improved the prediction accuracy (yielding an RMSE of $0.78, \mathrm{~m}^{2} \mathrm{~m}^{-2}$ ) over merely using OLI data (RMSE $=0.83, \mathrm{~m}^{2} \mathrm{~m}^{-2}$ ). The relationship between estimated and measured LAI using the ANN model for different scenarios is shown in Figure 5.9. As can be seen from the figure, the accuracy is higher than when only vegetation indices are used. 


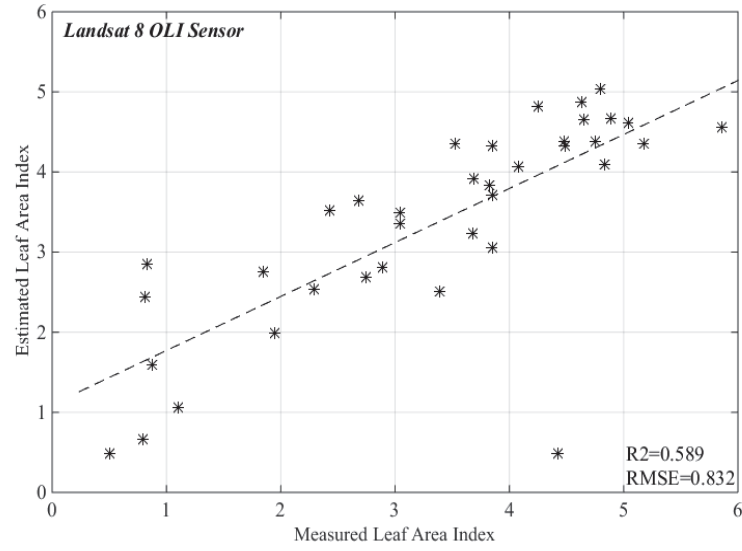

(a)

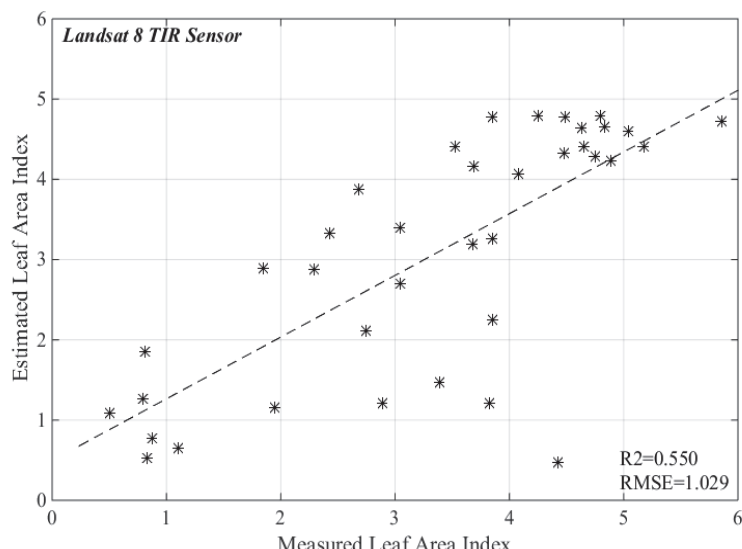

(b)

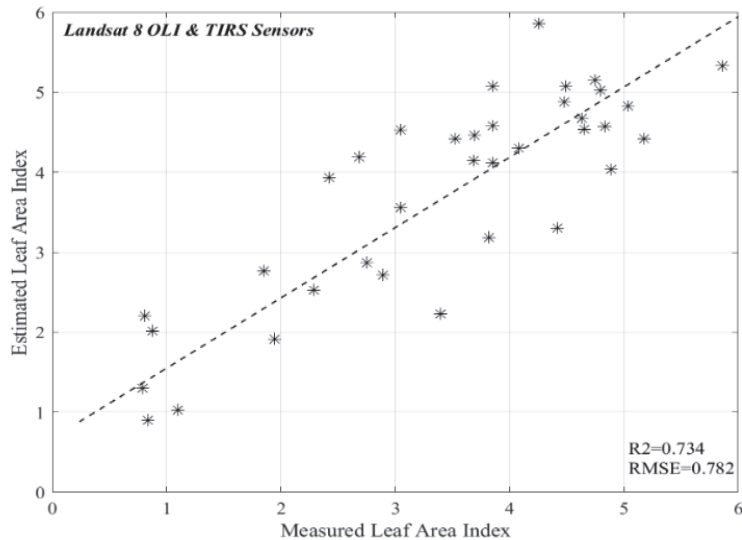

(c)

Figure 5.9: Scatterplot of measured versus estimated leaf area index using different input networks: OLI bands (a), TIRS bands (b), OLI + TIRS bands (c). 


\subsection{Discussion}

This study attempts to highlight the importance of satellite TIR data for the retrieval of LAI. The integration of TIR and VNIR/SWIR data is shown to improve the prediction of a vegetation biophysical parameter such as LAI. Our results further revealed that, apart from information regarding emissivity of soil and vegetation, also the accurate estimation of $\mathrm{Pv}$ is indispensable for the retrieval of LSE using NDVI ${ }^{\mathrm{THM}}$.

Previously, Sobrino et al. (2002) concluded that the NDVI ${ }^{\mathrm{THM}}$ produced promising results in retrieving LSE. To apply $\mathrm{NDVI}^{\mathrm{THM}}$ prior knowledge about soil and vegetation emissivities is essential (Becker and Li 1995; Li et al. 2013; Van de Griend and Owe 1993). Review of the literature revealed that $\mathrm{P}_{\mathrm{V}}$ has traditionally been estimated from remote sensing data using empirical relations with vegetation indices (e.g. NDVI and VARIgreen). In this study, a more accurate estimation of $\mathrm{Pv}$ is obtained using an ANN method, than using vegetation indices such as VARIgreen and NDVI $\left(\mathrm{R}^{2}=0.40, \mathrm{RMSE}=\right.$ 0.06 for NDVI, not shown). This confirms earlier conclusions by Boyd et al. (2002), who stated that the ANN approach is preferred for the retrieval of $\mathrm{P}_{V}$ as the ANN technique offers a powerful means of analyzing complex datasets without making assumptions about them. The result presented in Table 5.5 indicates that there is a significance difference between the LSE of plots harboring different vegetation types. However, as can be observed from Figure 5.6, the LSE difference was not distinct between mixed plots and Beech plots if the LAI value was similar. This can probably be explained by the fact that in mixed plots the Beech canopies play a dominant role in emitted radiance, hence emissivity, due to the size and angles of Beech leaves. Another explanation could be the limited number of bands in the TIR domain as well as the complexity of the emissivity of the plant's canopy due to the canopy cavity effect (Fuchs and Tanner 1966; Jin and Liang 2006). This result confirms the outcome of an earlier study (Neinavaz et al. 2016a) that canopies with differing structures but similar LAI values have dissimilar emissivities. Our results also confirm the conclusions of an earlier study by (Jacquemoud et al. 2009) that plants with planophile leaves have a higher reflectance in VNIR/SWIR regions. Our findings are also in agreement with the findings of Ullah et al. (2012a), who proved the concept of discrimination of vegetation using TIR hyperspectral data at leaf level. In general, this result is very promising regarding the extension of the present TIR remote sensing concept 
towards discriminating plant species at canopy level and over large areas, as well as monitoring LAI by means of TIR data. However, further experiments will be required using advanced TIR hyperspectral sensors.

Recently, LAI has been successfully retrieved using TIR hyperspectral data under controlled laboratory conditions with multivariate and univariate statistical techniques (Neinavaz et al. 2016c). This study revealed that LAI might be predicted using LSE calculated from multispectral satellite data, though with less accuracy. This may partly be explained by the use of broadband sensors and multispectral data, which mask the information usually available in the narrow spectral bands (Curran 1989; Schlerf et al. 2005). Additionally, review of the literature has shown that hyperspectral data are more efficient in providing extra information than multispectral data when quantifying vegetation characteristics (Cho et al. 2007; Mutanga and Skidmore 2004; Thenkabail et al. 2000; Zarco-Tejada et al. 2002). Moreover, an earlier paper by Neinavaz et al. (2016c) revealed that the LAI was predicted with the highest accuracy using the 10-12 $\mu \mathrm{m}$ wavelength range in combination with the bands in the $8-11 \mu \mathrm{m}$ range. Therefore, having only two TIR bands (Table 5.1) in the atmospheric window between $10 \mu \mathrm{m}$ and $12 \mu \mathrm{m}$ for Landsat 8 may have reduced prediction accuracy of the LAI.

The results of this study are in agreement with the recent finding of Neinavaz et al. (2016c) that there is no precedence using vegetation indices to predict LAI by means of TIR hyperspectral data. A possible explanation could be the little contrast observed in emissivity spectra over the TIR domain compared to the reflectance spectra (Hapke 2012). In addition, the combination of bands from different regions demonstrates higher sensitivity than one or two bands from a particular part of the electromagnetic spectrum for the prediction of vegetation parameters, since each band contains relevant and efficient information (Asrar et al. 1984; Bannari et al. 1995). Therefore, vegetation indices developed in this study only applied two broadband spectral over TIR region which appears are not sufficient to retrieve LAI. This result was further confirmed when a combination of both TIRS and OLI sensors data were employed to predict LAI using an ANN approach (Figure 5.9).

The results of this study reveal that the estimation of LAI using a trained ANN $\left(\mathrm{R}^{2}=0.55, \mathrm{RMSE}_{\mathrm{CV}}=1.02,\left(\mathrm{~m}^{2} \mathrm{~m}^{-2}\right)\right.$ for TIRS data $)$ is more reliable and achieves a higher prediction accuracy than the use of vegetation indices $\left(\mathrm{R}^{2}=\right.$ 
$0.44, \mathrm{R}^{2} \mathrm{CV}=0.35, \mathrm{RMSE}_{\mathrm{CV}}=1.202$ ). Therefore, this study not only shows that LAI is predictable using TIR remotely sensed data, but also that ANN approaches improve model accuracy compared to univariate techniques for estimating vegetation characteristics over TIR domain. Thus, that there is potential for the operational retrieval of LAI from remote sensing (Danson et al. 2003; Darvishzadeh et al. 2008b; Neinavaz et al. 2016c).

\subsection{Conclusion}

The possibility to predict LAI using TIR data in a mixed temperate forest (i.e. Bavarian Forest National Park) has been investigated in this study. We demonstrate the potential of using TIR data to predict LAI by using empirical (i.e. six vegetation indices) and machine learning (i.e. ANN) approaches. $\mathrm{P}_{\mathrm{V}}$, one of the important parameters to drive LSE (using NDVI ${ }^{\mathrm{THM}}$ ), is estimated with reasonable accuracy using an ANN technique. Positive correlations have been found between LSE and vegetation type in plots, and it has been observed that plots with different vegetation types have different LSE even when their LAI value is similar. This is a promising result and could form the basis for further investigation. The results demonstrate that using LSE, LAI can be predicted with reasonable accuracy. All six investigated indices achieved similar results predicting LAI using LSE. The ANN-based models serve as a reliable approach for estimating LAI, although the use of hyperspectral data from the TIR region needs to be investigated further. Predicted and measured LAI revealed a linear relationship in all considered models. Our findings suggest that combining VNIR/SWIR and TIR data might improve the prediction accuracy for estimating LAI. For the first time, it has been shown that even under non-controlled conditions prediction of LAI may be possible using TIR data. TIR remotely sensed data are once again revealed to be valuable in vegetation remote sensing studies. Further research is desirable, using TIR hyperspectral data (i.e. airborne or space-borne data) coupled with a canopy radiative transfer model. This study offers practical techniques for estimating LAI, an essential biodiversity variable, and thus will be valuable for the assessment of biodiversity and ecosystem services. 
Chapter 6

Synthesis: Sensing Vegetation Canopies in the Thermal Domain 


\subsection{Introduction}

Accurate estimation of vegetation characteristics is essential to understand changes in the terrestrial ecosystem fully. Remote sensing is a cost-effective, non-destructive, and repetitive technique which is the appropriate approach to monitor and assess ecosystems changes in comparison to the traditional destructive methods which are tedious, time-consuming, data lagged and expensive. In this respect, in the current thesis, two vegetation characteristics namely, leaf area index (LAI) as a vegetation biophysical variable and water content as a vegetation biochemical variable were addressed and quantified using thermal infrared remote sensing data (TIR, 8-14 $\mu \mathrm{m}$ ).

LAI is an important vegetation biophysical variable which plays a critical role in processes such as transpiration, and evaporation (Chen et al. 2005b) as well as in multiple crop process models (Duchemin et al. 2006). An accurate prediction of LAI is critically important as an input parameter of ecosystem process models. Additionally, temporal and spatial monitoring of LAI can improve our understanding of changes in forest ecosystems (Zheng and Moskal 2009). As a result, LAI has recently been identified as one of the crucial essential biodiversity variables (EBVs) among hundreds of variables which were potentially fit for satellite monitoring of progress towards the Aichi Biodiversity Targets (Pereira et al. 2013; Skidmore et al. 2015).

Fire occurrence is another EBV proposed to monitor ecosystem function using satellite data (Pereira et al. 2013; Skidmore et al. 2015). Evaluation of wildfire occurrence can be performed using fuel moisture content (FMC) and equivalent water thickness (EWT) by characterizing water status of the foliage (Chuvieco et al. 2004; Danson and Bowyer 2004). FMC is highly correlated with the risk of wildfire (Chuvieco et al. 2004; Hunt Jr et al. 2013). Moreover, influence biochemical processes in vegetation, such as photosynthesis and evaporation, are restricted by vegetation water content in terms of EWT (Peñuelas et al. 1994). Accordingly, in this thesis, the canopy water content in terms of FMC as mass-based and EWT as area-based variables is addressed and evaluated at the canopy level.

In the past decades, the use of visible-near infrared and shortwave infrared (VNIR-SWIR; 0.3-2.5 $\mu \mathrm{m}$ ) hyperspectral and multispectral remote sensing data for quantifying vegetation parameters at leaf, canopy, and landscape levels has been broadly studied (Asner and Martin 2008; Asner et al. 2003; 
Atzberger et al. 2010; Baret and Guyot 1991; Baret et al. 1989; Darvishzadeh et al. 2008a; Darvishzadeh et al. 2008b; Mirzaie et al. 2014). However, TIR data has not been perused properly in remote sensing vegetation studies, due to the low signal to noise ratio, difficulty in accurately estimating emissivity spectra as well as the complexity of emissivity spectra (Gerhards et al. 2016; Ribeiro da Luz and Crowley 2007; Ullah et al. 2012a).

\subsection{Measuring the response of canopy emissivity spectra to leaf area index variation using thermal hyperspectral data}

A few spectroscopic studies exist which focus on emissivity spectra link to vegetation characteristics at the leaf level (Buitrago et al. 2016; Ribeiro da Luz and Crowley 2007; Ullah et al. 2014). Although, the response of canopy reflectance spectra to LAI variation in the VNIR and SWIR regions has been addressed in the literature (Asner 1998; Asrar et al. 1984; Darvishzadeh et al. 2009), the response of canopy emissivity spectra to the variation of LAI values was not known and, yet, had to be considered.

Chapter 2 explored the response of canopies with different LAI values versus canopy emissivity spectra of various plant species over the TIR region. It revealed that there is a positive correlation between canopy emissivity spectra and LAI. However, the highest correlation values between LAI and individual emissivity spectra were different among the studied species and were observed in various parts of the TIR domain. The findings of Chapter 2 demonstrated that emissivity rises with increasing LAI values and this increment is more pronounced after the wavelength $9 \mu \mathrm{m}$ portion of the electromagnetic spectrum (Figure 6.1 and Figure 6.2). Additionally, the changes in canopy emissivity spectra value became less noticeable for the LAI values larger than $4.0\left(\mathrm{~m}^{2} \mathrm{~m}^{-2}\right)$. It was further observed that slight changes in LAI values (i.e. $<0.5 \mathrm{~m}^{2} \mathrm{~m}^{-2}$ ) are not readily remarkable through canopy emissivity spectra.

Our results from Chapter 2 indicated that while LAI values of different plant species were similar, canopy emissivity spectra of considered plant species were found to be statistically different at various wavebands and were also relatively distinctive. This finding suggests that LAI is presumably not the sole variable that effects emissivity spectra over TIR region and probably the 
interaction between LAI and other variables can also contribute to emissivity spectra differences in plant species. Previously, Ullah et al. (2012a) revealed that band $9.4 \mu \mathrm{m}$, among a few other wavebands, contains sufficient information to discriminate between different plant species at leaf level in the TIR domain. Similarly, our results from Chapter 2 demonstrated that the possible wavelengths over TIR region provides adequate information to distinguish among various plant species while the LAI value is similar is located in the wavelength between $8 \mu \mathrm{m}$ and $10 \mu \mathrm{m}$ (i.e. $8.0-8.3 \mu \mathrm{m}, 8.8-9.1$ $\mu \mathrm{m}, 9.4-9.6 \mu \mathrm{m}$, and 9.9-10.1 $\mu \mathrm{m}$ domains).

\subsection{Retrieval of leaf area index in different plant species using thermal hyperspectral data}

Some studies indicated that LAI value saturates at approximately $5.0-5.5\left(\mathrm{~m}^{2}\right.$ $\mathrm{m}^{-2}$ ) (Asner et al. 2003; Gower et al. 1999) while other studies illustrated that LAI reached a saturation level at roughly $2.5-3.0\left(\mathrm{~m}^{2} \mathrm{~m}^{-2}\right)$ using vegetation indices from multispectral data (Carlson et al. 1990; Sellers 1987). Consequently, vegetation indices calculated from multispectral data displayed a reduced sensitivity to LAI at high LAI values. Recently, Schlerf et al. (2005) and Darvishzadeh et al. (2008b) confirmed that narrow-band vegetation indices could predict LAI with better accuracy in comparison to broadband vegetation indices over VNIR and SWIR regions. However, prediction of LAI has not been researched in the TIR spectrum, and to our knowledge, it was revealed for the first time (in Chapter 3) that LAI could be predicted with moderate accuracy using TIR hyperspectral data under controlled laboratory conditions. 


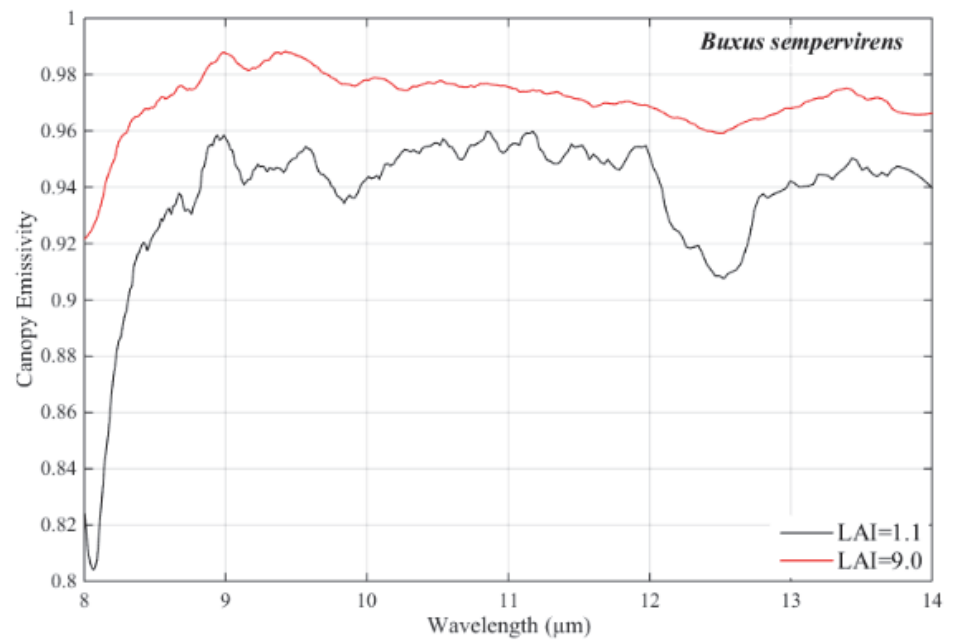

(a)

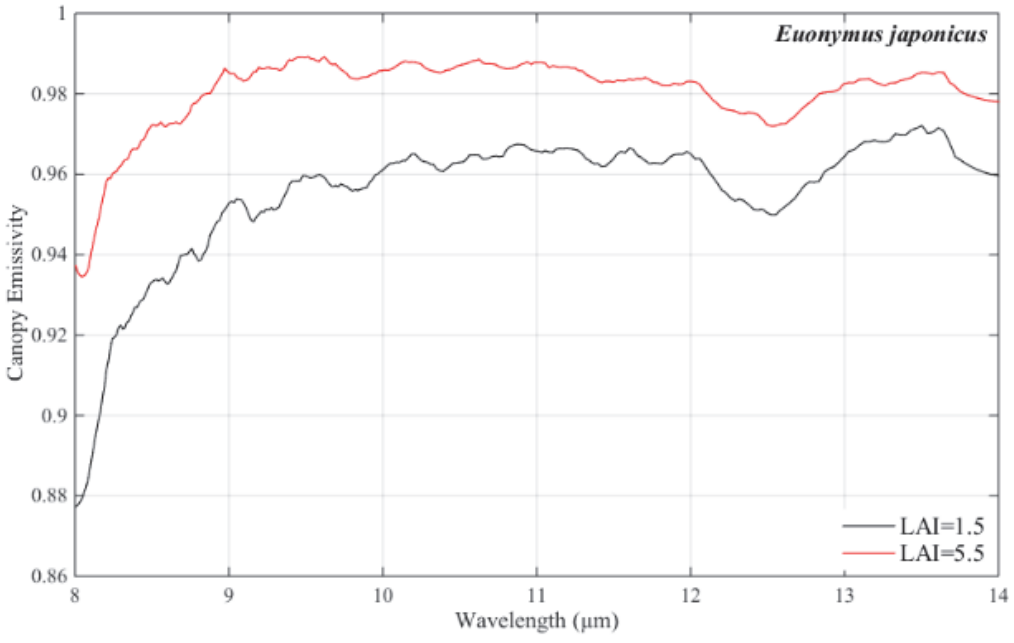

(b)

Figure 6.1: Effects of leaf area index variability on nadir-viewed canopy emissivity in the Buxus sempervirens (a), and Euonymus japonicus (b). 

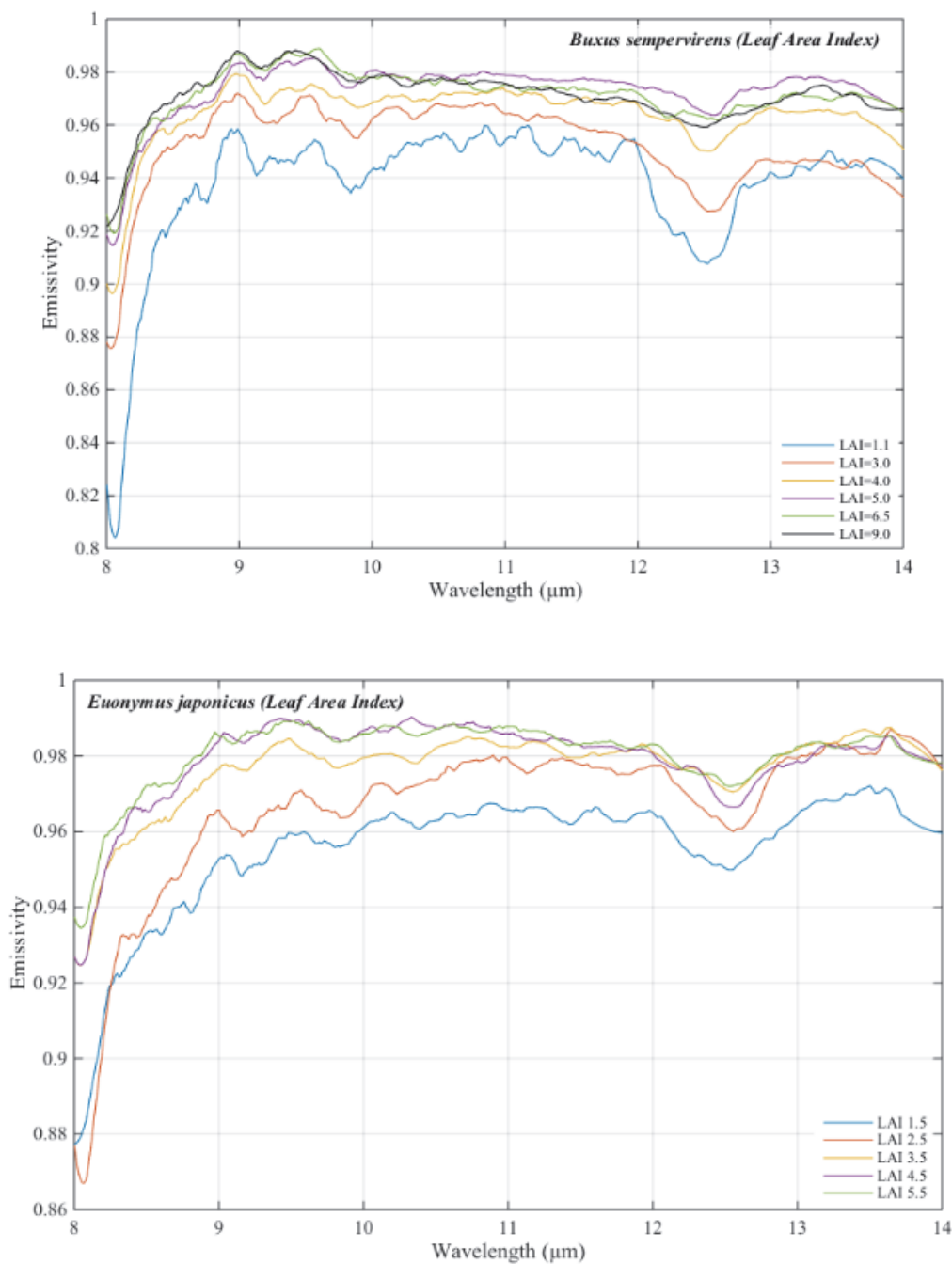

(b)

Figure 6.2: Changes in canopy emissivity spectra become smaller when LAI values exceed $4.0\left(\mathrm{~m}^{2} \mathrm{~m}^{-2}\right)$ in the Buxus sempervirens (a), and Euonymus japonicus (b).

Chapter 3 of this thesis discusses the potential of TIR hyperspectral data for the retrieval of LAI for four species which were structurally different. Several issues were considered regarding retrieval of LAI using TIR emissivity spectra. The correlation between LAI and the canopy emissivity spectra of individual wavebands was first determined. The results showed that the correlation between LAI and the emissivity spectra were not statistically significant for all wavebands. There was a difference in the highest correlation 
values between LAI and emissivity spectra in various parts of the TIR domain among the considered plant species (Figure 6.3).

The results of Chapter 3 demonstrated that LAI is predictable with moderate accuracy from emissivity spectra using vegetation indices even at relatively high LAI values (LAI > 5.5). It must be noted that different vegetation indices obtained similar results. The most sensitive waveband combination (i.e. wavebands yielded a high coefficient of determination in relation with LAI) across species was obtained by means of the 10-12 $\mu \mathrm{m}$ range in combination with the bands in the $8-11 \mu \mathrm{m}$ range. The improvements in the prediction accuracy of LAI were further confirmed when partial least square regression (PLSR) was applied compared to vegetation indices. Further, the LAI for all species were retrieved using artificial neural networks (ANN) as a machine learning approach. The results demonstrated that the accuracy of the retrieved LAI using ANN was comparable to those from vegetation indices as well as PLSR, as estimated in Chapter 3 (Table 6.1). The findings of Chapter 3 demonstrated that no matter which statistical method had been applied, the predicted accuracy was highly dependent on the plant species and was different among species. The LAI predicted from the pooled data was much less accurate compared to the LAI values estimated for individual species which may be attributed to the biochemical properties of leaves and the canopy structure of the studied species. 

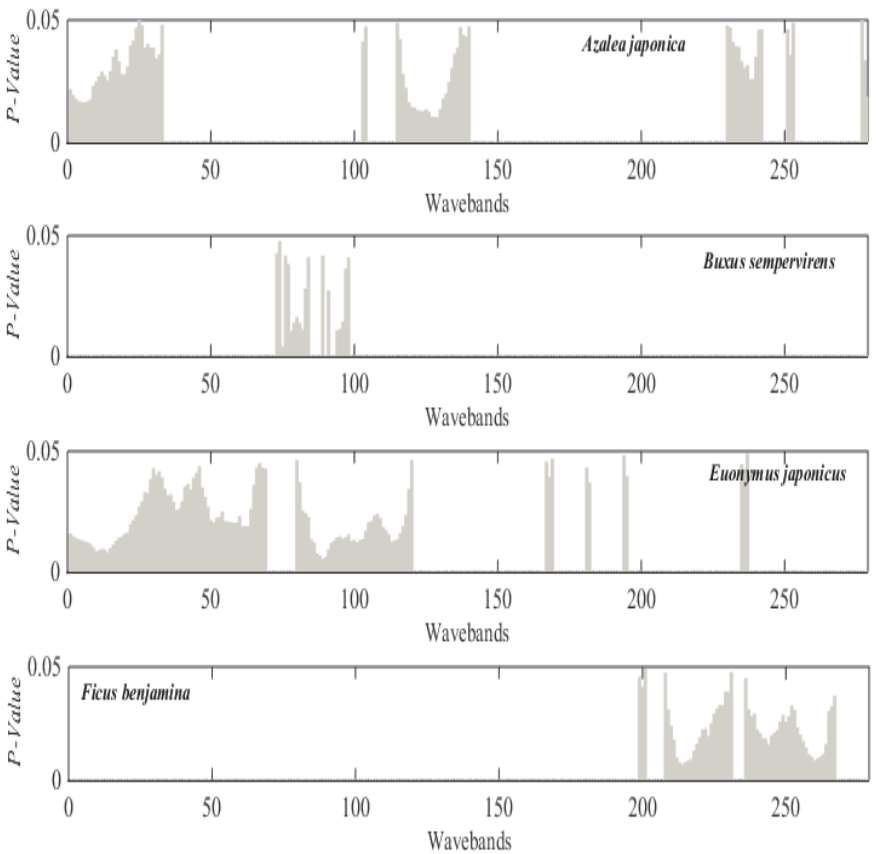

Figure 6.3: The relationship between canopy emissivity spectra at individual wavebands and leaf area index. The gray area represents the wavebands that were significantly different.

Table 6.1: Performance of simple ratio index (SR), partial least squares regression (PLSR), and artificial neural networks (ANN) models for predicting leaf area index.

\begin{tabular}{ccccccc}
\hline \multirow{2}{*}{ Species name } & \multicolumn{2}{c}{ SR } & \multicolumn{2}{c}{ PLSR } & \multicolumn{2}{c}{ ANN } \\
\cline { 2 - 7 } & $\mathbf{R}^{\mathbf{2}} \mathbf{C V}$ & $\mathbf{R M S E c v}(\%)$ & $\mathbf{R}^{\mathbf{2}}$ & RMSEcv (\%) & $\mathbf{R}^{\mathbf{2}} \mathbf{C V}$ & RMSEcv (\%) \\
\hline Ficus benjamina & 0.60 & 31.90 & 0.68 & 31.26 & 0.95 & 11.54 \\
Azalea japonica & 0.38 & 27.71 & 0.54 & 19.32 & 0.85 & 14.93 \\
$\begin{array}{c}\text { Euonymus } \\
\text { japonicus }\end{array}$ & 0.57 & 36.77 & 0.82 & 34.06 & 0.86 & 22.54 \\
Buxus sempervirens & 0.34 & 40.98 & 0.64 & 27.40 & 0.67 & 31.23 \\
\hline
\end{tabular}

\subsection{Retrieving vegetation canopy water content from hyperspectral thermal measurements}

The retrieval of vegetation water content is essential in assessing a drought risk (Peñuelas and Filella 1998), the risk of forest fire (Chuvieco et al. 2004; Chuvieco et al. 2002) as well as for the monitoring of ecosystem functions 
(Skidmore et al. 2015). Estimating of FMC and EWT, both at leaf and canopy levels, are widely investigated using VNIR and SWIR hyperspectral and multispectral data with a varying degree of success (Ceccato et al. 2002a; Clevers et al. 2010; Mirzaie et al. 2014). In contrast, the potential of TIR hyperspectral data to predict vegetation water content has hardly been considered at the canopy level. Recent technological development in the TIR spectroscopy offers an opportunity to assess the potential of the emissivity spectral for prediction of vegetation water content, particularly at canopy level over TIR region (hereafter, FMC and EWT at canopy level referred to as $\mathrm{FMC}_{\mathrm{C}}$ and $\mathrm{EWT}_{\mathrm{C}}$, respectively). The potential of the TIR domain to predict monitor $\mathrm{FMC}_{\mathrm{C}}$ and $\mathrm{EWT}_{\mathrm{C}}$ can improve and may be applicable for assessing drought risk and estimation of a wildfire occurrences.

Chapter 4 assesses the potential of TIR hyperspectral data for the estimation of FMC as mass-based vegetation water content and EWT as an area-based vegetation water content at canopy level, coupled with multivariate techniques. For upscaling of vegetation variables from leaf to canopy level, LAI is required and irreplaceable. The results in Chapter 4 demonstrated that both variables could be predicted accurately from TIR emissivity spectra using PLSR and ANN models. However, $\mathrm{FMC}_{\mathrm{C}}$ as a mass-based variable could be more accurately retrieved across species compared to EWTC as an area-based variable (Figure 6.4). This could be associated with the relationship between heat flow and mass which could incidentally denote the temperature, light use efficiency, and characterizes the water availability in the plant. The findings of Chapter 4 complement the knowledge from VNIR and SWIR regions in terms of prediction of canopy vegetation water content. The results confirmed that among the most important wavebands which contain sufficient information regarding prediction of $\mathrm{FMC}_{\mathrm{C}}$ and $\mathrm{EWT}_{\mathrm{C}}$, waveband $8.8 \mu \mathrm{m}$ is common to all the studied plant species. Additionally, the results demonstrated that ANN approach is superior when retrieving FMC $_{C}$ and EWTC compared to the PLSR models, which use TIR hyperspectral data and result in a relatively higher prediction accuracy. It was observed that the strength of the relation between PLSR and ANN models for prediction of $\mathrm{EWT}_{\mathrm{C}}$ and $\mathrm{FMC}_{\mathrm{C}}$ differed among studied plant species. This result is in agreement with the findings of Chapter 3 where it was revealed that the prediction accuracy of LAI using TIR data was highly dependent on the plant species. The results showed that for the retrieval of the considered 
variables (i.e. EWTC and $\mathrm{FMC}_{\mathrm{C}}$ ) for the pooled data using the most important wavebands identified by PLSR models as the network inputs for ANN were insufficient, and consequently, more wavebands were required to improve the prediction accuracy. However, the findings of this chapter once again confirmed that emissivity spectra over TIR region are likely to be speciesspecific since when the data of the studied plant species was pooled together, the prediction accuracies dropped.

\subsection{Successful retrieval of leaf area index from thermal satellite imagery over mixed temperate forest}

Satellite-based prediction of LAI has been widely addressed by means of VNIR and SWIR remote sensing data (Bacour et al. 2006; Colombo et al. 2003; Wang et al. 2004). However, TIR satellite data has yet to be considered to assess the relationship between vegetation biophysical variable, such as LAI and TIR data at the landscape level. In this respect, Chapter 5 assessed the relation between LAI and land surface emissivity (LSE) benefiting from Landsat-8 TIR data coupled with empirical and machine learning approaches.

This chapter has also attempted to highlight an important issue regarding the normalized difference vegetation index threshold method (NDVI ${ }^{\mathrm{THM}}$ ) for calculating LSE; and showed that besides knowledge regarding emissivity of soil and vegetation, an accurate prediction of the proportion of vegetation cover $\left(\mathrm{P}_{\mathrm{V}}\right)$ is crucial. The prediction accuracy of $\mathrm{P}_{\mathrm{V}}$ which was achieved by different approach yields different results and were highly dependent on the choice of approach $\left(\mathrm{R}^{2}=0.64, \mathrm{RMSE}=0.05\right.$ for $\mathrm{ANN} ; \mathrm{R}^{2}=0.42, \mathrm{RMSE}=$ 0.06 for VARIgreen; $\mathrm{R}^{2}=0.40$, RMSE $=0.06$ for NDVI) (Figure 6.5 and Figure 6.6). Accordingly, it could have considerable influence on the prediction accuracy of variables which were further calculated or retrieved using LSE. 

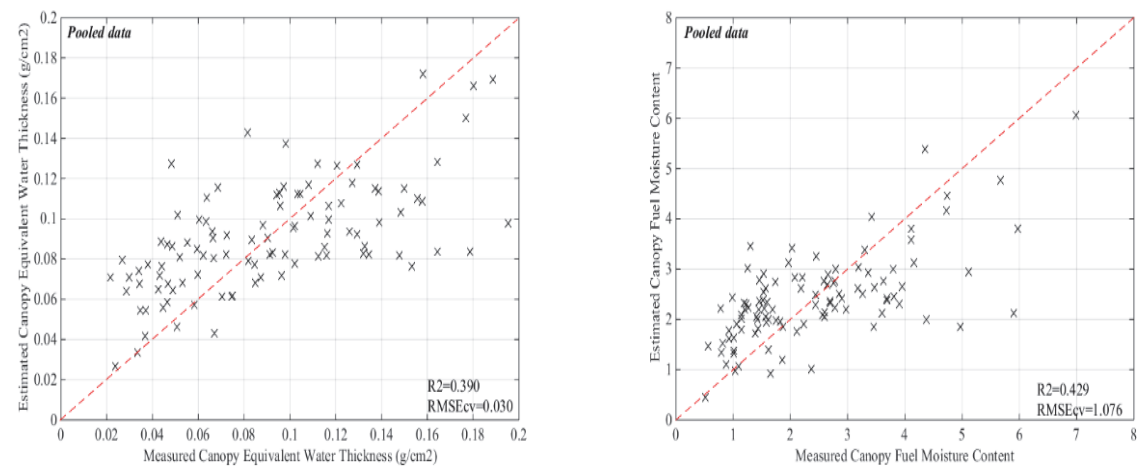

(a)
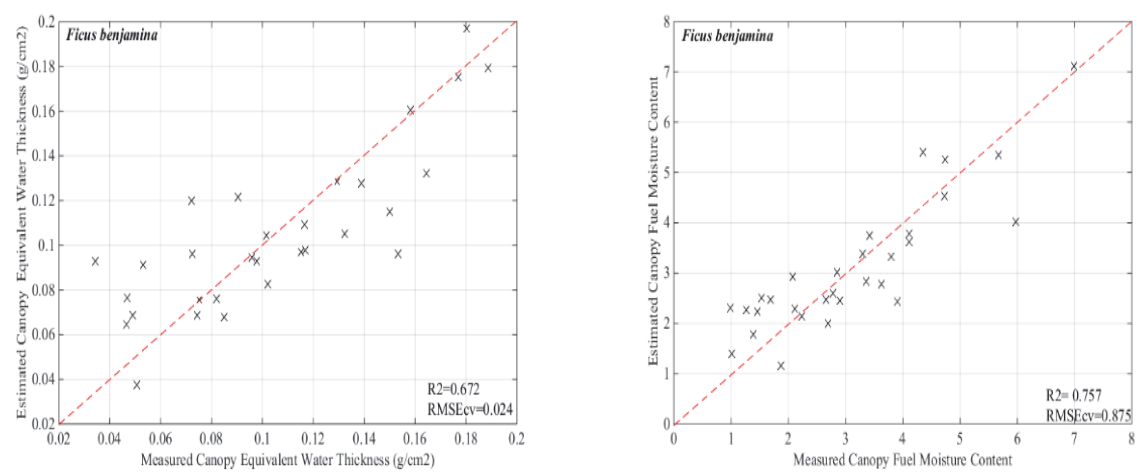

(b)
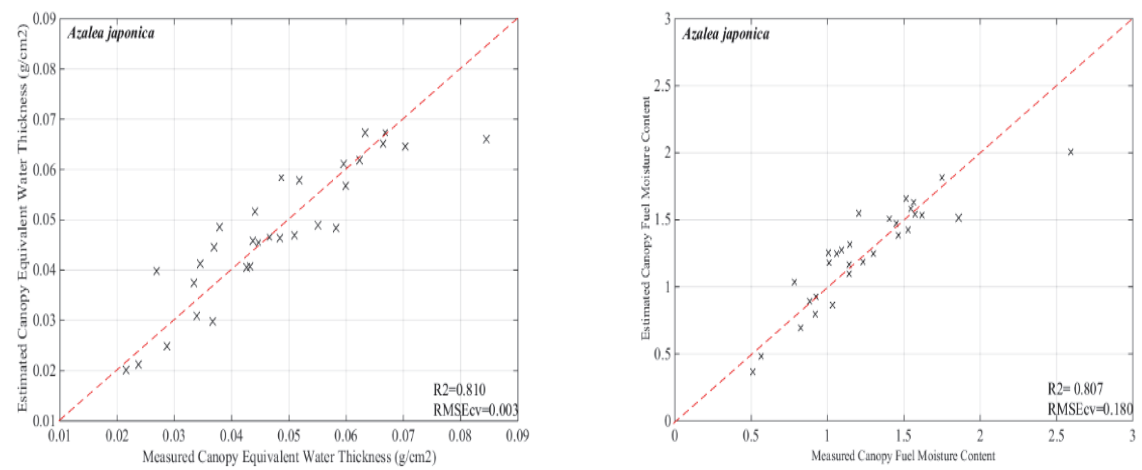

(c)

Figure 6.4: Scatterplots of measured versus estimated canopy equivalent water thickness (left side diagrams), and canopy fuel moisture content (right side diagrams) for the pooled data (a), Ficus benjamina (b), and Azalea japonica (c) using partial least square regression. 


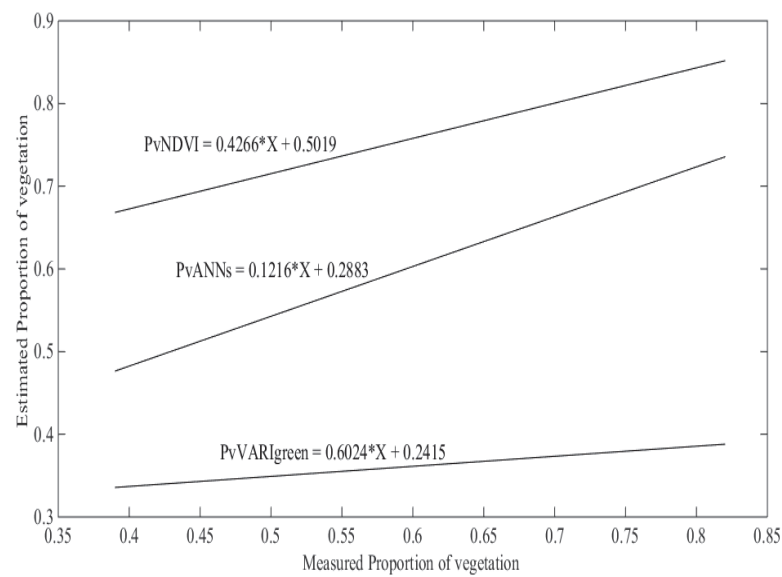

Figure 6.5: Comparison of the first-order polynomial fit obtained from different approaches for prediction of the proportion of vegetation cover $\left(\mathrm{P}_{\mathrm{V}}\right)$ in the Bavarian forest national park: normalized difference vegetation index $\left(\mathrm{P}_{\mathrm{V}}{ }^{\mathrm{NDVI}}\right)$; variable atmospherically resistant index $\left(\mathrm{P}_{\mathrm{V}}{ }^{\text {VARIgreen }}\right)$; artificial neural networks $\left(\mathrm{P}_{\mathrm{V}}{ }^{\mathrm{ANN}}\right)$.

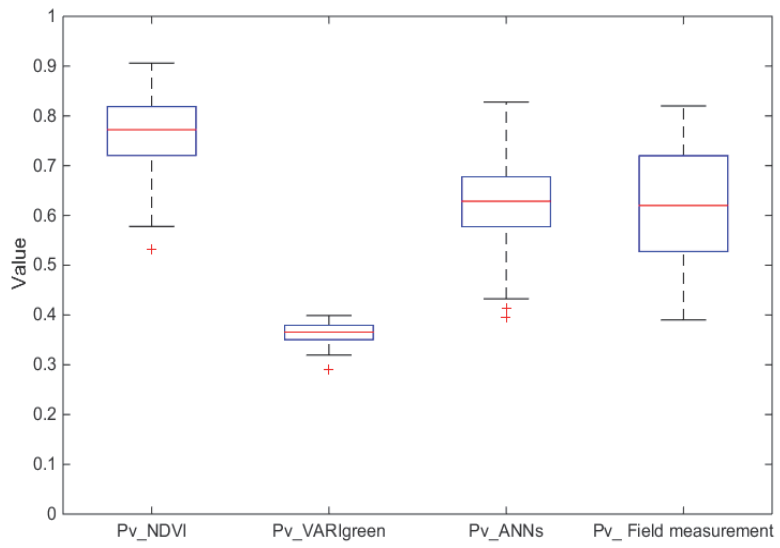

Figure 6.6: Median, lower and upper quartile values of the proportion of vegetation $\left(\mathrm{P}_{\mathrm{V}}\right)$ calculated using three different methods: normalized difference vegetation index $\left(\mathrm{P}_{\mathrm{V}}{ }^{\text {NDVI }}\right)$; variable atmospherically resistant index $\left(\mathrm{P}_{\mathrm{V}}\right.$ VARIgreen $)$; artificial neural networks $\left(\mathrm{P}_{\mathrm{V}}{ }^{\mathrm{ANN}}\right)$; and in situ measurements.

The findings of Chapter 5 demonstrated that a positive correlation exists between LAI and LSE and it was observed that LSE is influenced by increasing LAI values (Figure 6.7). As can be observed from Figure 6.7, the LSE was calculated from bands 10 and 11 of Landsat 8 varied with a LAI variation. This confirmed our earlier results obtained in Chapter 2, which 
demonstrated that by increasing LAI value the canopy emissivity spectra increases.

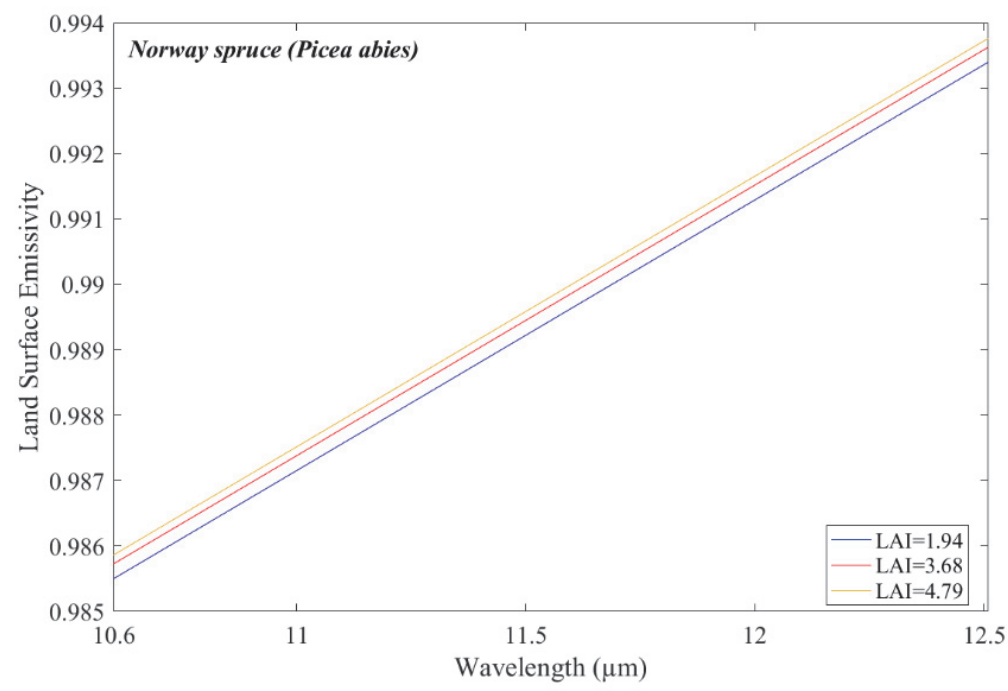

Figure 6.7: The land surface emissivity in Norway spruce (Picea abies) over a thermal infrared region corresponding to variation in leaf area index (LAI, $\left.\mathrm{m}^{2} \mathrm{~m}^{-2}\right)$.

In addition, the results of this chapter revealed that a statistically significant difference exists between plot's LSE and different vegetation types (Figure 5.7) except between Beech and mixed plots (i.e. including Beech and Spruce). The exception (i.e. vegetation type) can be partly explained by the differences in canopy structural parameters and concentrations of biochemical contents among different species as well as by having a limited number of (only two) TIR bands which are located between $10 \mu \mathrm{m}$ and $12 \mu \mathrm{m}$. Previously, Ullah et al. (2012a) found that wavebands bands $9.44 \mu \mathrm{m}, 12.71 \mu \mathrm{m}$, and $13.70 \mu \mathrm{m}$ have adequate information to discriminate among plant species at the leaf level.

Moreover, the results of this chapter confirmed the findings of the previous chapter (i.e. Chapter 3), which revealed that there is no preference to use vegetation indices to predict LAI using TIR data. A possible explanation could be the little contrast observed in absorption bands in emissivity spectra compared to the reflectance spectra (Hapke 2012). The most common remote sensing vegetation indices are developed using different bands located in various parts of the electromagnetic spectrum (Chen and Cihlar 1996; 
Gitelson et al. 2002; Pearson and Miller 1972; Rouse et al. 1974; Viña et al. 2011). Accordingly, the combination of bands from different regions usually demonstrates better sensitivity to vegetation variables since their information are complementary rather than bands from a particular part of the electromagnetic spectrum (Asrar et al. 1984; Bannari et al. 1995).

The results of this chapter confirmed that the retrieval of LAI from TIR data using an ANN approach is more accurate than the use of vegetation indices and confirmed our previous findings that multivariate techniques were the better choice in order to improve the prediction accuracy compared to univariate methods for retrieval of vegetation biophysical parameters over VNIR/SWIR and TIR regions (Danson et al. 2003; Neinavaz et al. 2016c). Further, for the first time, it was demonstrated that the combination of VNIR/SWIR and TIR data could enhance the prediction accuracy for estimation of vegetation biophysical parameters such as LAI (Figure 6.8).

\subsection{Border implications of using thermal infrared remotely sensed data for estimation of vegetation parameters in ecological studies}

The intention in this thesis was to explore the potential of TIR remote sensing data for prediction of LAI as a primary vegetation biophysical variable and canopy vegetation water content with a focus on empirical models. Different statistical techniques such as univariate versus multivariate approaches were applied for retrieval of vegetation characteristics from the laboratory to satellite level. 


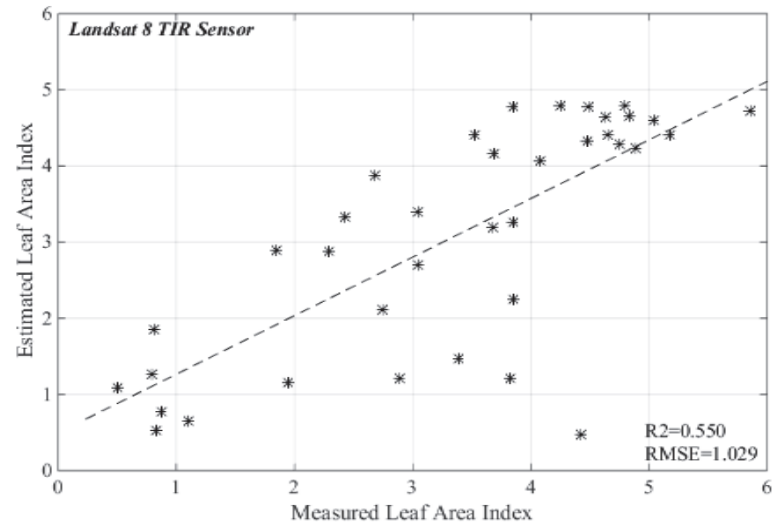

(a)

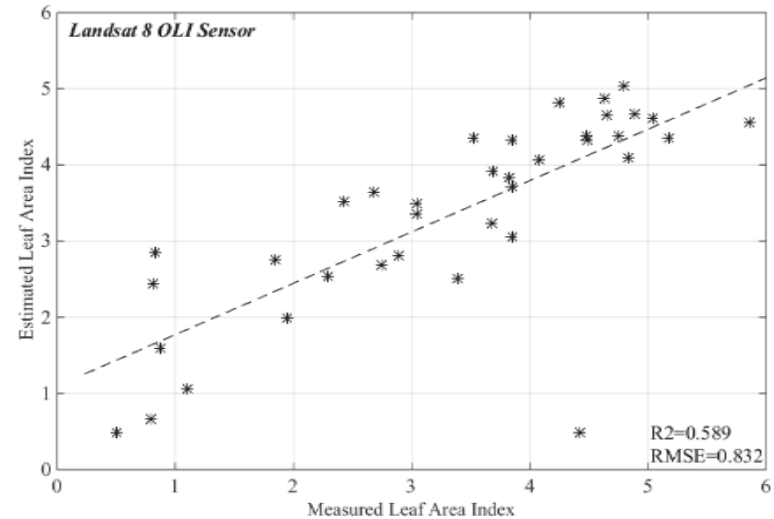

(b)

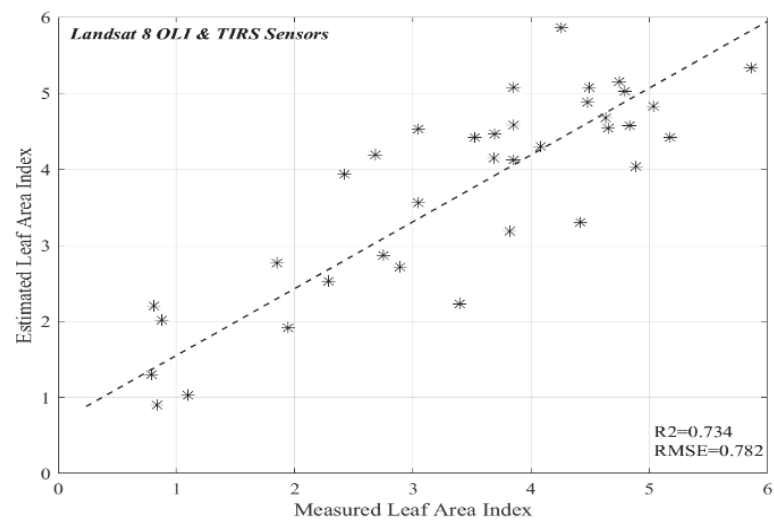

(c)

Figure 6.8: Scatterplot of measured versus estimated leaf area index using the artificial neural network and Landsat 8 bands from TIRS sensor (a), OLI sensor (b), and OLI and TIRS sensors (c). 
The research in this thesis assessed the capability of predicting vegetation variables using TIR, which remotely sensed data at the canopy level. In this respect, Chapter 2 investigated the effect of LAI values on emissivity spectra and proved for the first time that by raising LAI values, emissivity (of spectra) increases. The findings revealed that there are a number of other variables which have an influence upon the emissivity spectra. Chapter 3 predicted LAI using univariate and multivariate statistical techniques and demonstrated that LAI could be estimated with reasonable accuracy, even at relatively high LAI values using TIR hyperspectral data. Chapter 4 assessed the possibility of estimation vegetation water content in terms of FMC and EWT, both at canopy level from emissivity spectra and revealed that FMC at canopy level could be retrieved more accurately than EWT at canopy level. In other words, emissivity spectra were a better predictor when retrieving mass-based canopy water content. Additionally, ANN as a machine learning approach serves as an accurate technique for estimating considered variables in this thesis, although, its transferability desires to investigate using another TIR datasets. Chapter 5 explored the possibility of the estimation of LAI benefiting from Landsat 8 TIR data as well as presented how the results from Chapters 2 and 3 can be up-scaled. The results confirmed that LAI could be predicted with modest accuracy using an empirical approach. Additionally, the findings of this chapter demonstrated that a combination of reflectance and emissivity spectra might improve the prediction accuracy of LAI.

As a consequence, the findings of the thesis suggested that the information contained in TIR data can be complementary to other remote sensing data and can be counted as a new approach for the retrieval of vegetation biophysical and biochemical variables in further studies. Accurate estimation of vegetation parameters such as LAI and canopy water content are valuable in a variety of ecological and agricultural applications. The regional and global maps of LAI or vegetation water content benefiting from TIR data could be generated when new satellites (containing multiple thermal bands) such as HyspIRI (Lee et al. 2015) and space-based instrument such as ECOSTRESS (Johnson and Hook 2016) become operational. Consequently, such studies would make provision for expansion of EBVs for assessing and conserving biodiversity, especially in fragile ecosystems. Additionally, mapping canopy water content using TIR data will improve our conception of the water stress in plants through measuring land surface temperature and land surface 
emissivity (Karnieli et al. 2010; Wan et al. 2004), particularly for climatesensitive biomes. This would also determine how variation in vegetation water content impact the biophysical processes of terrestrial ecosystems (e.g. global carbon cycle).

\subsection{Future research avenues}

The potential of TIR hyperspectral data for predicting vegetation parameters at canopy level is novel and are not entirely investigated. However, the findings of this thesis enlighten the use of the TIR remote sensing in vegetation studies and underline the potential of TIR data for retrieval of vegetation variables at canopy level. In line with the statistical approaches and variables considered in this dissertation, further studies could be undertaken along three lines: examining the retrieval of other vegetation variables, using another dataset with different measurement conditions, and utilizing radiative transfer models in the TIR region using TIR hyperspectral data.

In this study, LAI and canopy water content have been addressed as two primary vegetation parameters in the ecosystem process models as well as EBVs (Pereira et al. 2013). Whereas, there are other vegetation parameters (e.g. specific leaf area, dry matter content, nitrogen, etc.) which must be considered in ecological studies and monitoring of ecosystems by means of remote sensing, in particular, TIR data (Skidmore et al. 2015). Therefore, other vegetation traits could be discussed similarly in future research.

Vegetation of the Bavarian Forest National Park is mainly dominated by three species consisting of Norway spruce (Picea abies), European beech (Fagus sylvatica), and Silver fir (Abies alba). Therefore, the application of using TIR remotely sensed data to other ecosystems, and different vegetation types are required to be considered in future studies.

In this study, in Chapters 2, 3 and 4 TIR hyperspectral data have been applied, while for Chapter 5 due to the unavailability of hyperspectral data in the TIR domain, TIR multispectral data have been used. Further research is crucial to explore the TIR hyperspectral remotely sensed data by means of space-borne and airborne platforms with fine resolutions. Another possible extension of this study would be on the use of information achieved by empirical approaches to parameterize the physical models such as radiative transfer models. 


\section{Bibliography}

Abdel-Rahman, E.M., Mutanga, O., Odindi, J., Adam, E., Odindo, A., \& Ismail, R. (2014). A comparison of partial least squares (PLS) and sparse PLS regressions for predicting yield of Swiss chard grown under different irrigation water sources using hyperspectral data. Computers and Electronics in Agriculture, 106, 11-19

Abdi, H. (2003). Partial least square regression (PLS regression). In (pp. $792-$ 795): Thousand Oaks, CA: Sage

Abdi, H. (2010). Partial least squares regression and projection on latent structure regression (PLS Regression). Wiley Interdisciplinary Reviews: Computational Statistics, 2, 97-106

Adler-Golden, S.M., Matthew, M.W., Bernstein, L.S., Levine, R.Y., Berk, A., Richtsmeier, S.C., Acharya, P.K., Anderson, G.P., Felde, J.W., \& Gardner, J. (1999). Atmospheric correction for shortwave spectral imagery based on MODTRAN4. In, SPIE's International Symposium on Optical Science, Engineering, and Instrumentation (pp. 61-69): International Society for Optics and Photonics

Ali, I., Greifeneder, F., Stamenkovic, J., Neumann, M., \& Notarnicola, C. (2015). Review of machine learning approaches for biomass and soil moisture retrievals from remote sensing data. Remote Sensing, 7, 16398-16421

Asner, G.P. (1998). Biophysical and biochemical sources of variability in canopy reflectance. Remote Sensing of Environment, 64, 234-253

Asner, G.P., Keller, M., Pereira Jr, R., Zweede, J.C., \& Silva, J.N. (2004). Canopy damage and recovery after selective logging in Amazonia: field and satellite studies. Ecological Applications, 14, 280-298

Asner, G.P., \& Martin, R.E. (2008). Spectral and chemical analysis of tropical forests: Scaling from leaf to canopy levels. Remote Sensing of Environment, $112,3958-3970$

Asner, G.P., Scurlock, J.M., \& A Hicke, J. (2003). Global synthesis of leaf area index observations: implications for ecological and remote sensing studies. Global Ecology and Biogeography, 12, 191-205

Asrar, G., Fuchs, M., Kanemasu, E., \& Hatfield, J. (1984). Estimating absorbed photosynthetic radiation and leaf area index from spectral reflectance in wheat. Agronomy journal, 76, 300-306

Atkinson, P.M., \& Tatnall, A. (1997). Introduction neural networks in remote sensing. International Journal of Remote Sensing, 18, 699-709 
Atzberger, C. (2004). Object-based retrieval of biophysical canopy variables using artificial neural nets and radiative transfer models. Remote Sensing of Environment, 93, 53-67

Atzberger, C., Guérif, M., Baret, F., \& Werner, W. (2010). Comparative analysis of three chemometric techniques for the spectroradiometric assessment of canopy chlorophyll content in winter wheat. Computers and Electronics in Agriculture, 73, 165-173

Bacour, C., Baret, F., Béal, D., Weiss, M., \& Pavageau, K. (2006). Neural network estimation of LAI, fAPAR, fCover and LAI $\times \mathrm{C}$ ab, from top of canopy MERIS reflectance data: principles and validation. Remote Sensing of Environment, 105, 313-325

Bannari, A., Morin, D., Bonn, F., \& Huete, A. (1995). A review of vegetation indices. Remote sensing reviews, 13, 95-120

Barclay, H.J. (1998). Conversion of total leaf area to projected leaf area in lodgepole pine and Douglas-fir. Tree Physiology, 18, 185-193

Baret, F., \& Guyot, G. (1991). Potentials and limits of vegetation indices for LAI and APAR assessment. Remote Sensing of Environment, 35, 161-173

Baret, F., Guyot, G., \& Major, D. (1989). TSAVI: a vegetation index which minimizes soil brightness effects on LAI and APAR estimation. In, Geoscience and Remote Sensing Symposium, 1989. IGARSS'89. 12th Canadian Symposium on Remote Sensing., 1989 International (pp. 13551358): IEEE

Becker, F., \& Li, Z.-L. (1990). Temperature-independent spectral indices in thermal infrared bands. Remote Sensing of Environment, 32, 17-33

Becker, F., \& Li, Z.L. (1995). Surface temperature and emissivity at various scales: Definition, measurement and related problems. Remote sensing reviews, 12, 225-253

Boegh, E., Søgaard, H., Broge, N., Hasager, C., Jensen, N., Schelde, K., \& Thomsen, A. (2002). Airborne multispectral data for quantifying leaf area index, nitrogen concentration, and photosynthetic efficiency in agriculture. Remote Sensing of Environment, 81, 179-193

Bowyer, P., \& Danson, F. (2004). Sensitivity of spectral reflectance to variation in live fuel moisture content at leaf and canopy level. Remote Sensing of Environment, 92, 297-308

Boyd, D., Foody, G., \& Ripple, W. (2002). Evaluation of approaches for forest cover estimation in the Pacific Northwest, USA, using remote sensing. Applied Geography, 22, 375-392 
Bréda, N.J. (2003). Ground-based measurements of leaf area index: a review of methods, instruments and current controversies. Journal of Experimental Botany, 54, 2403-2417

Breunig, F.M., Galvão, L.S., Formaggio, A.R., \& Couto, E.G. (2009). The combined use of reflectance, emissivity and elevation Aster/Terra data for tropical soil studies. Revista Brasileira de Ciência do Solo, 33, 1785-1794

Brilis, G.M., Van Waasbergen, R., Stokely, P., \& Gerlach, C. (2001). Remote Sensing Tools Assist in Environmental Forensics: Part II--Digital Tools. Environmental Forensics, 2, 223-229

Broge, N.H., \& Mortensen, J.V. (2002). Deriving green crop area index and canopy chlorophyll density of winter wheat from spectral reflectance data. Remote Sensing of Environment, 81, 45-57

Broich, M., Hansen, M., Stolle, F., Potapov, P., Margono, B.A., \& Adusei, B. (2011). Remotely sensed forest cover loss shows high spatial and temporal variation across Sumatera and Kalimantan, Indonesia 2000-2008. Environmental Research Letters, 6, 014010

Brown, L., Chen, J.M., Leblanc, S.G., \& Cihlar, J. (2000). A shortwave infrared modification to the simple ratio for LAI retrieval in boreal forests: An image and model analysis. Remote Sensing of Environment, 71, 16-25

Brutsaert, W. (2013). Evaporation into the atmosphere: theory, history and applications. Springer Science \& Business Media

Buitrago, M.F., Groen, T.A., Hecker, C.A., \& Skidmore, A.K. (2016). Changes in thermal infrared spectra of plants caused by temperature and water stress. ISPRS Journal of Photogrammetry and Remote Sensing, 111, 22-31

Campbell, J.B., \& Wynne, R.H. (2011). Introduction to remote sensing. Guilford Press

Carlson, T.N., Perry, E.M., \& Schmugge, T.J. (1990). Remote estimation of soil moisture availability and fractional vegetation cover for agricultural fields. Agricultural and Forest Meteorology, 52, 45-69

Carter, G.A., \& Knapp, A.K. (2001). Leaf optical properties in higher plants: linking spectral characteristics to stress and chlorophyll concentration. American Journal of Botany, 88, 677-684

Ceccato, P., Flasse, S., \& Grégoire, J.-M. (2002a). Designing a spectral index to estimate vegetation water content from remote sensing data: Part 2. validation and applications. Remote Sensing of Environment, 82, 198-207

Ceccato, P., Flasse, S., Tarantola, S., Jacquemoud, S., \& Grégoire, J.-M. (2001). Detecting vegetation leaf water content using reflectance in the optical domain. Remote Sensing of Evironment, 77, 22-33 
Ceccato, P., Gobron, N., Flasse, S., Pinty, B., \& Tarantola, S. (2002b). Designing a spectral index to estimate vegetation water content from remote sensing data: Part 1: Theoretical approach. Remote Sensing of Environment, 82, 188197

Chen, D., Huang, J., \& Jackson, T.J. (2005a). Vegetation water content estimation for corn and soybeans using spectral indices derived from MODIS near-and short-wave infrared bands. Remote Sensing of Environment, 98, 225-236

Chen, G., Bui, T.D., Quach, K.G., \& Qian, S.-E. (2014). Denoising hyperspectral imagery using principal component analysis and block-matching 4D filtering. Canadian Journal of Remote Sensing, 40, 60-66

Chen, J.M. (1996). Evaluation of vegetation indices and a modified simple ratio for boreal applications. Canadian Journal of Remote Sensing, 22, 229-242

Chen, J.M., Chen, X., Ju, W., \& Geng, X. (2005b). Distributed hydrological model for mapping evapotranspiration using remote sensing inputs. Journal of Hydrology, 305, 15-39

Chen, J.M., \& Cihlar, J. (1996). Retrieving leaf area index of boreal conifer forests using Landsat TM images. Remote Sensing of Environment, 55, 153162

Chen, J.M., Plummer, P.S., Rich, M., Gower, S.T., \& Norman, J.M. (1997). Leaf area index measurements. Journal of geophysical research, 102, 29-429

Cheng, Y.-B., Zarco-Tejada, P.J., Riaño, D., Rueda, C.A., \& Ustin, S.L. (2006). Estimating vegetation water content with hyperspectral data for different canopy scenarios: Relationships between AVIRIS and MODIS indexes. Remote Sensing of Environment, 105, 354-366

Chin, W.W., Marcolin, B.L., \& Newsted, P.R. (2003). A partial least squares latent variable modeling approach for measuring interaction effects: Results from a Monte Carlo simulation study and an electronic-mail emotion/adoption study. Information systems research, 14, 189-217

Cho, M., Skidmore, A., \& Atzberger, C. (2008). Towards red-edge positions less sensitive to canopy biophysical parameters for leaf chlorophyll estimation using properties optique spectrales des feuilles (PROSPECT) and scattering by arbitrarily inclined leaves (SAILH) simulated data. International Journal of Remote Sensing, 29, 2241-2255

Cho, M.A., Skidmore, A., Corsi, F., Van Wieren, S.E., \& Sobhan, I. (2007). Estimation of green grass/herb biomass from airborne hyperspectral imagery using spectral indices and partial least squares regression. International journal of applied Earth observation and geoinformation, 9, 414-424 
Chuvieco, E., Aguado, I., Cocero, D., \& Riano, D. (2003). Design of an empirical index to estimate fuel moisture content from NOAA-AVHRR images in forest fire danger studies. International Journal of Remote Sensing, 24, 1621-1637

Chuvieco, E., Cocero, D., Riano, D., Martin, P., Martınez-Vega, J., de la Riva, J., \& Perez, F. (2004). Combining NDVI and surface temperature for the estimation of live fuel moisture content in forest fire danger rating. Remote Sensing of Environment, 92, 322-331

Chuvieco, E., Deshayes, M., Stach, N., Cocero, D., \& Riaño, D. (1999). Shortterm fire risk: foliage moisture content estimation from satellite data. Remote Sensing of Large Wildfires (pp. 17-38): Springer

Chuvieco, E., Riano, D., Aguado, I., \& Cocero, D. (2002). Estimation of fuel moisture content from multitemporal analysis of Landsat Thematic Mapper reflectance data: applications in fire danger assessment. International Journal of Remote Sensing, 23, 2145-2162

Claudio, H.C., Cheng, Y., Fuentes, D.A., Gamon, J.A., Luo, H., Oechel, W., Qiu, H.-L., Rahman, A.F., \& Sims, D.A. (2006). Monitoring drought effects on vegetation water content and fluxes in chaparral with the $970 \mathrm{~nm}$ water band index. Remote Sensing of Environment, 103, 304-311

Clerbaux, C., Drummond, J.R., Flaud, J.-M., \& Orphal, J. (2011). Using Thermal Infrared Absorption and Emission to Determine Trace Gases. The Remote Sensing of Tropospheric Composition from Space (pp. 123-151): Springer

Clevers, J., \& Kooistra, L. (2006). Using spectral information at the NIR water absorption features to estimate canopy water content and biomass. In, ISPRS Mid-term Symposium Remote Sensing: from pixels to processes, Enschede, The Netherlands (pp. 8-11)

Clevers, J.G., Kooistra, L., \& Schaepman, M.E. (2010). Estimating canopy water content using hyperspectral remote sensing data. International journal of applied Earth observation and geoinformation, 12, 119-125

Colombo, R., Bellingeri, D., Fasolini, D., \& Marino, C.M. (2003). Retrieval of leaf area index in different vegetation types using high resolution satellite data. Remote Sensing of Environment, 86, 120-131

Colombo, R., Meroni, M., Marchesi, A., Busetto, L., Rossini, M., Giardino, C., $\&$ Panigada, C. (2008). Estimation of leaf and canopy water content in poplar plantations by means of hyperspectral indices and inverse modeling. Remote Sensing of Environment, 112, 1820-1834

Curran, P.J. (1989). Remote sensing of foliar chemistry. Remote Sensing of Environment, 30, 271-278 
Curran, P.J., Dungan, J.L., \& Peterson, D.L. (2001). Estimating the foliar biochemical concentration of leaves with reflectance spectrometry: testing the Kokaly and Clark methodologies. Remote Sensing of Environment, 76, 349-359

Danson, F., \& Bowyer, P. (2004). Estimating live fuel moisture content from remotely sensed reflectance. Remote Sensing of Environment, 92, 309-321

Danson, F., \& Plummer, S. (1995). Red-edge response to forest leaf area index. Remote Sensing, 16, 183-188

Danson, F., Rowland, C., \& Baret, F. (2003). Training a neural network with a canopy reflectance model to estimate crop leaf area index. International Journal of Remote Sensing, 24, 4891-4905

Danson, F., Steven, M., Malthus, T., \& Clark, J. (1992). High-spectral resolution data for determining leaf water content. International Journal of Remote Sensing, 13, 461-470

Darvishzadeh, R., Atzberger, C., Skidmore, A., \& Abkar, A. (2009). Leaf Area Index derivation from hyperspectral vegetation indicesand the red edge position. International Journal of Remote Sensing, 30, 6199-6218

Darvishzadeh, R., Skidmore, A., Atzberger, C., \& van Wieren, S. (2008a). Estimation of vegetation LAI from hyperspectral reflectance data: Effects of soil type and plant architecture. International journal of applied Earth observation and geoinformation, 10, 358-373

Darvishzadeh, R., Skidmore, A., Schlerf, M., Atzberger, C., Corsi, F., \& Cho, M. (2008b). LAI and chlorophyll estimation for a heterogeneous grassland using hyperspectral measurements. ISPRS Journal of Photogrammetry and Remote Sensing, 63, 409-426

Dash, P., Göttsche, F.-M., Olesen, F.-S., \& Fischer, H. (2002). Land surface temperature and emissivity estimation from passive sensor data: Theory and practice-current trends. International Journal of Remote Sensing, 23, 25632594

Daszykowski, M., Serneels, S., Kaczmarek, K., Van Espen, P., Croux, C., \& Walczak, B. (2007). TOMCAT: A MATLAB toolbox for multivariate calibration techniques. Chemometrics and intelligent laboratory systems, $85,269-277$

Dawson, T., Curran, P., North, P., \& Plummer, S. (1999). The propagation of foliar biochemical absorption features in forest canopy reflectance: A theoretical analysis. Remote Sensing of Environment, 67, 147-159

De Jong, S.M., Pebesma, E.J., \& Lacaze, B. (2003). Above-ground biomass assessment of Mediterranean forests using airborne imaging spectrometry: 
the DAIS Peyne experiment. International Journal of Remote Sensing, 24, 1505-1520

Deardorff, J. (1978). Efficient prediction of ground surface temperature and moisture, with inclusion of a layer of vegetation. Journal of Geophysical Research: Oceans, 83, 1889-1903

Delrot, S., Medrano, H., Or, E., Bavaresco, L., \& Grando, S. (2010). Methodologies and results in grapevine research. Springer

Desbois, N., Deshayes, M., \& Beudoin, A. (1997). Protocol for fuel moisture content measurements. A review of remote sensing methods for the study of large wildland fires, 61-72

Drake, B., Raschke, K., \& Salisbury, F. (1970). Temperature and transpiration resistances of Xanthium leaves as affected by air temperature, humidity, and wind speed. Plant Physiology, 46, 324-330

Du, Q., \& Fowler, J.E. (2007). Hyperspectral image compression using JPEG2000 and principal component analysis. Geoscience and Remote Sensing Letters, IEEE, 4, 201-205

Duchemin, B., Hadria, R., Erraki, S., Boulet, G., Maisongrande, P., Chehbouni, A., Escadafal, R., Ezzahar, J., Hoedjes, J., \& Kharrou, M. (2006). Monitoring wheat phenology and irrigation in Central Morocco: On the use of relationships between evapotranspiration, crops coefficients, leaf area index and remotely-sensed vegetation indices. Agricultural Water Management, 79, 1-27

Duda, R.O., \& Hart, P.E. (1973). Pattern classification and scene analysis. Wiley New York

Duro, D.C., Coops, N.C., Wulder, M.A., \& Han, T. (2007). Development of a large area biodiversity monitoring system driven by remote sensing. Progress in Physical Geography, 31, 235-260

Eisele, A., Chabrillat, S., Hecker, C., Hewson, R., Lau, I.C., Rogass, C., Segl, K., Cudahy, T.J., Udelhoven, T., \& Hostert, P. (2015). Advantages using the thermal infrared (TIR) to detect and quantify semi-arid soil properties. Remote Sensing of Environment, 163, 296-311

Elvidge, C.D. (1988). Thermal infrared reflectance of dry plant materials: 2.5$20.0 \mu \mathrm{m}$. Remote Sensing of Environment, 26, 265-285

Elvidge, C.D., \& Chen, Z. (1995). Comparison of broad-band and narrow-band red and near-infrared vegetation indices. Remote Sensing of Environment, $54,38-48$ 
Eriksson, H.M., Eklundh, L., Kuusk, A., \& Nilson, T. (2006). Impact of understory vegetation on forest canopy reflectance and remotely sensed LAI estimates. Remote Sensing of Environment, 103, 408-418

Fabre, S., Lesaignoux, A., Olioso, A., \& Briottet, X. (2011a). Influence of water content on spectral reflectance of leaves in the 3-15-domain. Geoscience and Remote Sensing Letters, IEEE, 8, 143-147

Fabre, S., Lesaignoux, A., Olioso, A., \& Briottet, X. (2011b). Influence of Wwater content on spectral reflectance of leaves in the 3-15-domain. Geoscience and Remote Sensing Letters, IEEE, 8, 143-147

Fernández, V., Guzmán-Delgado, P., Graça, J., Santos, S., \& Gil, L. (2016). Cuticle Structure in Relation to Chemical Composition: Re-assessing the Prevailing Model. Frontiers in plant science, 7

Fernández, V., Guzmán, P., Peirce, C.A., McBeath, T.M., Khayet, M., \& McLaughlin, M.J. (2014). Effect of wheat phosphorus status on leaf surface properties and permeability to foliar-applied phosphorus. Plant and soil, $384,7-20$

Foley, S., Rivard, B., Sanchez-Azofeifa, G.A., \& Calvo, J. (2006). Foliar spectral properties following leaf clipping and implications for handling techniques. Remote Sensing of Environment, 103, 265-275

French, A., Schmugge, T., \& Kustas, W. (2000). Discrimination of senescent vegetation using thermal emissivity contrast. Remote Sensing of Environment, 74, 249-254

Frey, C.M., \& Parlow, E. (2012). Flux measurements in Cairo. Part 2: On the determination of the spatial radiation and energy balance using ASTER satellite data. Remote sensing, 4, 2635-2660

Fuchs, M., \& Tanner, C. (1966). Infrared thermometry of vegetation. Agronomy journal, 58, 597-601

Gao, B.-C. (1996). NDWI-A normalized difference water index for remote sensing of vegetation liquid water from space. Remote Sensing of Environment, 58, 257-266

Gao, B.-C., \& Goetzt, A.F. (1995). Retrieval of equivalent water thickness and information related to biochemical components of vegetation canopies from AVIRIS data. Remote Sensing of Environment, 52, 155-162

Gao, X., Huete, A.R., Ni, W., \& Miura, T. (2000). Optical-biophysical relationships of vegetation spectra without background contamination. Remote Sensing of Environment, 74, 609-620 
Garnier, E., Laurent, G., Bellmann, A., Debain, S., Berthelier, P., Ducout, B., Roumet, C., \& Navas, M.L. (2001). Consistency of species ranking based on functional leaf traits. New Phytologist, 152, 69-83

Gates, D.M. (2012). Biophysical ecology. Courier Corporation

Gates, D.M., \& Tantraporn, W. (1952). The Reflectivity of Deciduous Trees and Herbaceous Plants in the Infrared to 25 Microns. Science (New York, NY), 115,613

Geladi, P., \& Kowalski, B.R. (1986). Partial least-squares regression: a tutorial. Analytica chimica acta, 185, 1-17

Geography, N.R.C.A.H.C.o. (1965). The Science of Geography: Report. National Academies

Gerber, F., Marion, R., Olioso, A., Jacquemoud, S., da Luz, B.R., \& Fabre, S. (2011). Modeling directional-hemispherical reflectance and transmittance of fresh and dry leaves from $0.4 \mu \mathrm{m}$ to $5.7 \mu \mathrm{m}$ with the PROSPECT-VISIR model. Remote Sensing of Environment, 115, 404-414

Gerhards, M., Rock, G., Schlerf, M., \& Udelhoven, T. (2016). Water stress detection in potato plants using leaf temperature, emissivity, and reflectance. International journal of applied Earth observation and geoinformation, 53, 27-39

Ghulam, A., Li, Z.-L., Qin, Q., Tong, Q., Wang, J., Kasimu, A., \& Zhu, L. (2007). A method for canopy water content estimation for highly vegetated surfacesshortwave infrared perpendicular water stress index. Science in China Series D: Earth Sciences, 50, 1359-1368

Gillespie, A., Rokugawa, S., Matsunaga, T., Cothern, J.S., Hook, S., \& Kahle, A.B. (1998). A temperature and emissivity separation algorithm for Advanced Spaceborne Thermal Emission and Reflection Radiometer (ASTER) images. IEEE Transactions on Geoscience and Remote Sensing, $36,1113-1126$

Gitelson, A.A., Kaufman, Y.J., Stark, R., \& Rundquist, D. (2002). Novel algorithms for remote estimation of vegetation fraction. Remote Sensing of Environment, 80, 76-87

Glover, B.J. (2000). Differentiation in plant epidermal cells. Journal of Experimental Botany, 51, 497-505

Goel, P.K. (2003). Hyper-spectral remote sensing for weed and nitrogen stress detection. In: McGill University Montreal, Canada

Gonzalez-Dugo, V., Zarco-Tejada, P., Nicolás, E., Nortes, P.A., Alarcón, J.J., Intrigliolo, D.S., \& Fereres, E. (2013). Using high resolution UAV thermal 
imagery to assess the variability in the water status of five fruit tree species within a commercial orchard. Precision Agriculture, 14, 660-678

Gower, S.T., Kucharik, C.J., \& Norman, J.M. (1999). Direct and indirect estimation of leaf area index, f APAR, and net primary production of terrestrial ecosystems. Remote Sensing of Environment, 70, 29-51

Gutman, G., \& Ignatov, A. (1998). The derivation of the green vegetation fraction from NOAA/AVHRR data for use in numerical weather prediction models. International Journal of Remote Sensing, 19, 1533-1543

Guzmán-Delgado, P., Graça, J., Cabral, V., Gil, L., \& Fernández, V. (2016). The presence of cutan limits the interpretation of cuticular chemistry and structure: Ficus elastica leaf as an example. Physiologia Plantarum, 157, 205-220

Haaland, D.M., \& Thomas, E.V. (1988). Partial least-squares methods for spectral analyses. 1. Relation to other quantitative calibration methods and the extraction of qualitative information. Analytical chemistry, 60, 11931202

Haboudane, D., Miller, J.R., Pattey, E., Zarco-Tejada, P.J., \& Strachan, I.B. (2004). Hyperspectral vegetation indices and novel algorithms for predicting green LAI of crop canopies: Modeling and validation in the context of precision agriculture. Remote Sensing of Environment, 90, 337-352

Hapke, B. (2012). Theory of reflectance and emittance spectroscopy. Cambridge university press

Helland, I.S. (1988). On the structure of partial least squares regression. Communications in statistics-Simulation and Computation, 17, 581-607

Herschel, W. (1800). Experiments on the refrangibility of the invisible rays of the sun. by william herschel, 11. dfrs. Philosophical Transactions of the Royal Society of London, 284-292

Heurich, M. (2008). Automatic recognition and measurement of single trees based on data from airborne laser scanning over the richly structured natural forests of the Bavarian Forest National Park. Forest Ecology and Management, 255, 2416-2433

Heurich, M., Beudert, B., Rall, H., \& Křenová, Z. (2010). National parks as model regions for interdisciplinary long-term ecological research: the Bavarian Forest and Šumavá National Parks underway to transboundary ecosystem research. Long-Term Ecological Research (pp. 327-344): Springer

Hikosaka, K. (2004). Interspecific difference in the photosynthesis-nitrogen relationship: patterns, physiological causes, and ecological importance. Journal of plant research, 117, 481-494 
Hikosaka, K., \& Anten, N.P. (2012). An evolutionary game of leaf dynamics and its consequences for canopy structure. Functional Ecology, 26, 1024-1032

Hirosawa, Y., Marsh, S.E., \& Kliman, D.H. (1996). Application of standardized principal component analysis to land-cover characterization using multitemporal AVHRR data. Remote Sensing of Environment, 58, 267-281

Holden, H., \& LeDrew, E. (1998). Spectral discrimination of healthy and nonhealthy corals based on cluster analysis, principal components analysis, and derivative spectroscopy. Remote Sensing of Environment, 65, 217-224

Hook, S.J., \& Kahle, A.B. (1996). The micro Fourier transform interferometer $(\mu$ FTIR) - a new field spectrometer for acquisition of infrared data of natural surfaces. Remote Sensing of Environment, 56, 172-181

Hori, M., Aoki, T., Tanikawa, T., Motoyoshi, H., Hachikubo, A., Sugiura, K., Yasunari, T.J., Eide, H., Storvold, R., \& Nakajima, Y. (2006). In-situ measured spectral directional emissivity of snow and ice in the $8-14 \mu \mathrm{m}$ atmospheric window. Remote Sensing of Environment, 100, 486-502

Hotelling, H. (1933). Analysis of a complex of statistical variables into principal components. Journal of educational psychology, 24, 417

Huber, C. (2005). Long lasting nitrate leaching after bark beetle attack in the highlands of the Bavarian Forest National Park. Journal of environmental quality, 34, 1772-1779

Huete, A., Didan, K., Miura, T., Rodriguez, E.P., Gao, X., \& Ferreira, L.G. (2002). Overview of the radiometric and biophysical performance of the MODIS vegetation indices. Remote Sensing of Environment, 83, 195-213

Hunt, E.R., \& Rock, B.N. (1989). Detection of changes in leaf water content using near-and middle-infrared reflectances. Remote Sensing of Environment, 30, 43-54

Hunt, E.R., Wang, L., Qu, J.J., \& Hao, X. (2012). Remote sensing of fuel moisture content from canopy water indices and normalized dry matter index. Journal of Applied Remote Sensing, 6, 061705-061705

Hunt Jr, E.R., Ustin, S.L., \& Riaño, D. (2013). Remote sensing of leaf, canopy, and vegetation water contents for satellite environmental data records. Satellite-based Publications on Climate Change (pp. 335-357): Springer

Jacquemoud, S., \& Baret, F. (1990). PROSPECT: A model of leaf optical properties spectra. Remote Sensing of Environment, 34, 75-91

Jacquemoud, S., Verhoef, W., Baret, F., Bacour, C., Zarco-Tejada, P.J., Asner, G.P., François, C., \& Ustin, S.L. (2009). PROSPECT+ SAIL models: A review of use for vegetation characterization. Remote Sensing of Environment, 113, S56-S66 
Jiménez-Muñoz, J.C., Sobrino, J.A., Gillespie, A., Sabol, D., \& Gustafson, W.T. (2006). Improved land surface emissivities over agricultural areas using ASTER NDVI. Remote Sensing of Environment, 103, 474-487

Jiménez-Muñoz, J.C., Sobrino, J.A., Plaza, A., Guanter, L., Moreno, J., \& Martínez, P. (2009). Comparison between fractional vegetation cover retrievals from vegetation indices and spectral mixture analysis: Case study of PROBA/CHRIS data over an agricultural area. Sensors, 9, 768-793

Jin, M., \& Liang, S. (2006). An improved land surface emissivity parameter for land surface models using global remote sensing observations. Journal of Climate, 19, 2867-2881

Jin, Y.-Q., \& Liu, C. (1997). Biomass retrieval from high-dimensional active/passive remote sensing data by using artificial neural networks. International Journal of Remote Sensing, 18, 971-979

Johnson, W.R., \& Hook, S.J. (2016). Mid and thermal infrared remote sensing at the Jet Propulsion Laboratory. In, SPIE Defense+ Security (pp. 98190H98190H-98198): International Society for Optics and Photonics

Jordan, C.F. (1969). Derivation of leaf area index from quality of light on the forest floor. Ecology, 663-666

Kahle, A.B. (1980). Surface thermal properties. Remote Sensing in Geology, 257273

Kahle, A.B., \& Alley, R.E. (1992). Separation of temperature and emittance in remotely sensed radiance measurements. Remote Sensing of Environment, $42,107-111$

Kalaitzidis, C., Heinzel, V., \& Zianis, D. (2010). A review of multispectral vegetation indices for biomass estimation. In, Imagin [e, g] Europe: Proceedings of the 29th Symposium of the European Association of Remote Sensing Laboratories, Chania, Greece (p. 201): IOS Press

Kampe, T.U., Asner, G.P., Green, R.O., Eastwood, M., Johnson, B.R., \& Kuester, M. (2010). Advances in airborne remote sensing of ecosystem processes and properties: toward high-quality measurement on a global scale. In, SPIE Optical Engineering+ Applications (pp. 78090J-78090J-78013): International Society for Optics and Photonics

Karnieli, A., Agam, N., Pinker, R.T., Anderson, M., Imhoff, M.L., Gutman, G.G., Panov, N., \& Goldberg, A. (2010). Use of NDVI and land surface temperature for drought assessment: merits and limitations. Journal of climate, 23, 618-633 
Kaufman, Y.J., \& Tanre, D. (1992). Atmospherically resistant vegetation index (ARVI) for EOS-MODIS. IEEE Transactions on Geoscience and Remote Sensing, 30, 261-270

Kerr, J.T., \& Ostrovsky, M. (2003). From space to species: ecological applications for remote sensing. Trends in Ecology \& Evolution, 18, 299305

Khayet, M., \& Fernández, V. (2012). Estimation of the solubility parameters of model plant surfaces and agrochemicals: a valuable tool for understanding plant surface interactions. Theoretical biology and medical modelling, 9, 1

Kimball, J., Keyser, A., Running, S., \& Saatchi, S. (2000). Regional assessment of boreal forest productivity using an ecological process model and remote sensing parameter maps. Tree Physiology, 20,761-775

Kimes, D. (1980). Effects of vegetation canopy structure on remotely sensed canopy temperatures. Remote Sensing of Environment, 10, 165-174

Kirkland, L., Herr, K., Keim, E., Adams, P., Salisbury, J., Hackwell, J., \& Treiman, A. (2002). First use of an airborne thermal infrared hyperspectral scanner for compositional mapping. Remote Sensing of Environment, 80, 447-459

Knoche, M., Beyer, M., Peschel, S., Oparlakov, B., \& Bukovac, M.J. (2004). Changes in strain and deposition of cuticle in developing sweet cherry fruit. Physiologia Plantarum, 120, 667-677

Koetz, B., Baret, F., Poilvé, H., \& Hill, J. (2005). Use of coupled canopy structure dynamic and radiative transfer models to estimate biophysical canopy characteristics. Remote Sensing of Environment, 95, 115-124

Kokaly, R.F., Asner, G.P., Ollinger, S.V., Martin, M.E., \& Wessman, C.A. (2009). Characterizing canopy biochemistry from imaging spectroscopy and its application to ecosystem studies. Remote Sensing of Environment, 113, S78-S91

Kokaly, R.F., \& Clark, R.N. (1999). Spectroscopic determination of leaf biochemistry using band-depth analysis of absorption features and stepwise multiple linear regression. Remote Sensing of Environment, 67, 267-287

Kooistra, L., Salas, E., Clevers, J., Wehrens, R., Leuven, R., Nienhuis, P., \& Buydens, L. (2004). Exploring field vegetation reflectance as an indicator of soil contamination in river floodplains. Environmental Pollution, 127, 281290

Korb, A.R., Dybwad, P., Wadsworth, W., \& Salisbury, J.W. (1996). Portable Fourier transform infrared spectroradiometer for field measurements of radiance and emissivity. Applied Optics, 35, 1679-1692 
Laurin, G.V., Chen, Q., Lindsell, J.A., Coomes, D.A., Del Frate, F., Guerriero, L., Pirotti, F., \& Valentini, R. (2014). Above ground biomass estimation in an African tropical forest with lidar and hyperspectral data. ISPRS Journal of Photogrammetry and Remote Sensing, 89, 49-58

Lee, C.M., Cable, M.L., Hook, S.J., Green, R.O., Ustin, S.L., Mandl, D.J., \& Middleton, E.M. (2015). An introduction to the NASA Hyperspectral InfraRed Imager (HyspIRI) mission and preparatory activities. Remote Sensing of Environment, 167, 6-19

Li, L., Ustin, S.L., \& Lay, M. (2005). Application of AVIRIS data in detection of oil-induced vegetation stress and cover change at Jornada, New Mexico. Remote Sensing of Environment, 94, 1-16

Li, Z.-L., Wu, H., Wang, N., Qiu, S., Sobrino, J.A., Wan, Z., Tang, B.-H., \& Yan, G. (2013). Land surface emissivity retrieval from satellite data. International Journal of Remote Sensing, 34, 3084-3127

Lillesand, T., Kiefer, R.W., \& Chipman, J. (2014). Remote sensing and image interpretation. John Wiley \& Sons

Lindroth, A., Lagergren, F., Aurela, M., Bjarnadottir, B., Christensen, T., Dellwik, E., Grelle, A., Ibrom, A., Johansson, T., \& Lankreijer, H. (2008). Leaf area index is the principal scaling parameter for both gross photosynthesis and ecosystem respiration of Northern deciduous and coniferous forests. Tellus B, 60, 129-142

Liu, J., Chen, J., Cihlar, J., \& Chen, W. (1999). Net primary productivity distribution in the BOREAS region from a process model using satellite and surface data. Journal of Geophysical Research: Atmospheres, 104, 2773527754

Liu, J., Chen, J., Cihlar, J., \& Park, W. (1997). A process-based boreal ecosystem productivity simulator using remote sensing inputs. Remote Sensing of Environment, 62, 158-175

Lloyd, J., Bloomfield, K., Domingues, T.F., \& Farquhar, G.D. (2013). Photosynthetically relevant foliar traits correlating better on a mass vs an area basis: of ecophysiological relevance or just a case of mathematical imperatives and statistical quicksand? New Phytologist, 199, 311-321

Matsushita, B., Xu, M., Chen, J., Kameyama, S., \& Tamura, M. (2004). Estimation of regional net primary productivity (NPP) using a process-based ecosystem model: How important is the accuracy of climate data? Ecological Modelling, 178, 371-388

McKinney, M.L., \& Schoch, R.M. (2003). Environmental science: Systems and solutions. Jones \& Bartlett Learning 
Mirzaie, M., Darvishzadeh, R., Shakiba, A., Matkan, A., Atzberger, C., \& Skidmore, A. (2014). Comparative analysis of different uni-and multivariate methods for estimation of vegetation water content using hyperspectral measurements. International journal of applied Earth observation and geoinformation, 26, 1-11

Morsdorf, F., Kötz, B., Meier, E., Itten, K., \& Allgöwer, B. (2006). Estimation of LAI and fractional cover from small footprint airborne laser scanning data based on gap fraction. Remote Sensing of Environment, 104, 50-61

Mushore, T.D., Mutanga, O., Odindi, J., \& Dube, T. (2016). Assessing the potential of integrated Landsat 8 thermal bands, with the traditional reflective bands and derived vegetation indices in classifying urban landscapes. Geocarto International, 1-34

Mutanga, O., \& Skidmore, A.K. (2004). Narrow band vegetation indices overcome the saturation problem in biomass estimation. International Journal of Remote Sensing, 25, 3999-4014

Myneni, R.B., Ramakrishna, R., Nemani, R., \& Running, S.W. (1997). Estimation of global leaf area index and absorbed PAR using radiative transfer models. Geoscience and Remote Sensing, IEEE Transactions on, 35, 1380-1393

Neinavaz, E., Darvishzadeh, R., Skidmore, A.K., \& Groen, T.A. (2016a). Measuring the response of canopy emissivity spectra to leaf area index variation using thermal hyperspectral data. International Journal of Applied Earth Observation and Geoinformation, 53, 40-47

Neinavaz, E., Darvishzadeh, R., Skidmore, A.K., \& Groen, T.A. (2016b). Measuring the response of canopy emissivity spectra to leaf areaindex variation using thermal hyperspectral data. International journal of applied Earth observation and geoinformation, 53, 40-47

Neinavaz, E., Skidmore, A.K., Darvishzadeh, R., \& Groen, T.A. (2016c). Retrieval of leaf area index in different plant species using thermal hyperspectral data. ISPRS Journal of Photogrammetry and Remote Sensing, $119,390-401$

Nowlan, S.J., \& Hinton, G.E. (1992). Simplifying neural networks by soft weight-sharing. Neural computation, 4, 473-493

Paliwal, M., \& Kumar, U.A. (2009). Neural networks and statistical techniques: A review of applications. Expert systems with applications, 36, 2-17

Paltridge, G., \& Barber, J. (1988). Monitoring grassland dryness and fire potential in Australia with NOAA/AVHRR data. Remote Sensing of Environment, 25, 381-394 
Pearson, R.L., \& Miller, L.D. (1972). Remote mapping of standing crop biomass for estimation of the productivity of the shortgrass prairie. In, Remote Sensing of Environment, VIII (p. 1355)

Peñuelas, J., \& Filella, I. (1998). Visible and near-infrared reflectance techniques for diagnosing plant physiological status. Trends in plant science, 3, 151156

Peñuelas, J., Gamon, J., Fredeen, A., Merino, J., \& Field, C. (1994). Reflectance indices associated with physiological changes in nitrogen-and water-limited sunflower leaves. Remote Sensing of Environment, 48, 135-146

Pereira, H.M., Ferrier, S., Walters, M., Geller, G.N., Jongman, R., Scholes, R.J., Bruford, M.W., Brummitt, N., Butchart, S., \& Cardoso, A. (2013). Essential biodiversity variables. Science, 339, 277-278

Peri, P.L. (2005). Leaf and canopy photosynthesis models for cocksfoot (Dactylis glomerata L.) grown in a silvopastoral system. Editorial Dunken

Pettorelli, N., Vik, J.O., Mysterud, A., Gaillard, J.-M., Tucker, C.J., \& Stenseth, N.C. (2005). Using the satellite-derived NDVI to assess ecological responses to environmental change. Trends in Ecology \& Evolution, 20, 503-510

Pettorelli, N., Wegmann, M., Skidmore, A., Mücher, S., Dawson, T.P., Fernandez, M., Lucas, R., Schaepman, M.E., Wang, T., \& O'Connor, B. (2016). Framing the concept of satellite remote sensing essential biodiversity variables: challenges and future directions. Remote Sensing in Ecology and Conservation

Pinol, J., Filella, I., Ogaya, R., \& Peñuelas, J. (1998). Ground-based spectroradiometric estimation of live fine fuel moisture of Mediterranean plants. Agricultural and Forest Meteorology, 90, 173-186

Pinter Jr, P.J., Jackson, R.D., Elaine Ezra, C., \& Gausman, H.W. (1985). Sunangle and canopy-architecture effects on the spectral reflectance of six wheat cultivars. International Journal of Remote Sensing, 6, 1813-1825

Poulter, B., \& Cramer, W. (2009). Satellite remote sensing of tropical forest canopies and their seasonal dynamics. International Journal of Remote Sensing, 30, 6575-6590

Prakash, A. (2000). Thermal remote sensing: concepts, issues and applications. International Archives of Photogrammetry and Remote Sensing, 33, 239243

Pruitt, E.L. (1970). Thoughts on inputs to information systems. The Professional Geographer, 22, 12-14 
Pu, R., Gong, P., \& Biging, G. (2003). Simple calibration of AVIRIS data and LAI mapping of forest plantation in southern Argentina. International Journal of Remote Sensing, 24, 4699-4714

Qi, Y., Dennison, P.E., Jolly, W.M., Kropp, R.C., \& Brewer, S.C. (2014). Spectroscopic analysis of seasonal changes in live fuel moisture content and leaf dry mass. Remote Sensing of Environment, 150, 198-206

Quattrochi, D.A., \& Luvall, J.C. (1999). Thermal infrared remote sensing for analysis of landscape ecological processes: methods and applications. Landscape ecology, 14, 577-598

Ramoelo, A., Skidmore, A.K., Cho, M.A., Schlerf, M., Mathieu, R., \& Heitkönig, I.M. (2012). Regional estimation of savanna grass nitrogen using the rededge band of the spaceborne RapidEye sensor. International journal of applied Earth observation and geoinformation, 19, 151-162

Reitberger, J., Krzystek, P., \& Heurich, M. (2006). Full-waveform analysis of small footprint airborne laser scanning data in the Bavarian forest national park for tree species classification. 3D Remote Sensing in Forestry, 218, 227

Riaño, D., Valladares, F., Condés, S., \& Chuvieco, E. (2004). Estimation of leaf area index and covered ground from airborne laser scanner (Lidar) in two contrasting forests. Agricultural and Forest Meteorology, 124, 269-275

Riaño, D., Vaughan, P., Chuvieco, E., Zarco-Tejada, P.J., \& Ustin, S.L. (2005). Estimation of fuel moisture content by inversion of radiative transfer models to simulate equivalent water thickness and dry matter content: analysis at leaf and canopy level. Geoscience and Remote Sensing, IEEE Transactions on, 43, 819-826

Ribeiro da Luz, B. (2006). Attenuated total reflectance spectroscopy of plant leaves: a tool for ecological and botanical studies. New Phytologist, 172, 305-318

Ribeiro da Luz, B., \& Crowley, J.K. (2007). Spectral reflectance and emissivity features of broad leaf plants: Prospects for remote sensing in the thermal infrared (8.0-14.0 $\mu \mathrm{m})$. Remote Sensing of Environment, 109, 393-405

Ribeiro da Luz, B., \& Crowley, J.K. (2010). Identification of plant species by using high spatial and spectral resolution thermal infrared $(8.0-13.5 \mu \mathrm{m})$ imagery. Remote Sensing of Environment, 114, 404-413

Richardson, A.J., \& Weigand, C. (1977). Distinguishing vegetation from soil background information. Photogrammetric engineering and remote sensing, 43

Rogalski, A. (2012). History of infrared detectors. Opto-Electronics Review, 20, 279-308 
Roujean, J.-L., \& Breon, F.-M. (1995). Estimating PAR absorbed by vegetation from bidirectional reflectance measurements. Remote Sensing of Environment, 51, 375-384

Rouse, J., Haas, R., Schell, J., Deering, D., \& Harlan, J. (1974). Monitoring the vernal advancements and retrogradation of natural vegetation. $N A S A / G S F C$, Final Report, Greenbelt, MD, USA, 1-137

Rubio, E., Caselles, V., \& Badenas, C. (1997). Emissivity measurements of several soils and vegetation types in the $8-14, \mu \mathrm{m}$ Wave band: Analysis of two field methods. Remote Sensing of Environment, 59, 490-521

Running, S.W., \& Coughlan, J.C. (1988). A general model of forest ecosystem processes for regional applications. I. Hydrologic balance, canopy gas exchange and primary production processes. Ecological Modelling, 42, 125154

Running, S.W., Nemani, R.R., Heinsch, F.A., Zhao, M., Reeves, M., \& Hashimoto, H. (2004). A continuous satellite-derived measure of global terrestrial primary production. Bioscience, 54, 547-560

Salimi Kouchi, H., Sahebi, M., Abkar, A., \& Valadan Zoej, M. (2013). Fractional Vegetation Cover Estimation In Urban Environments. ISPRS-International Archives of the Photogrammetry, Remote Sensing and Spatial Information Sciences, 1, 357-360

Salisbury, J. (1998). Spectral measurements field guide. Published by the Defense Technology Information Center as Report No. ADA362372. Earth Satellite Corporation

Salisbury, J., \& Milton, N. (1988). Thermal infrared (2.5-to 13.5-?? m) directional hemispherical reflectance of leaves. Photogrammetric engineering and remote sensing, 54, 1301-1304

Salisbury, J.W. (1986). Preliminary measurements of leaf spectral reflectance in the 8-14 $\mu \mathrm{m}$ region. International Journal of Remote Sensing, 7, 1879-1886

Salisbury, J.W., Wald, A., \& D'Aria, D.M. (1994). Thermal-infrared remote sensing and Kirchhoff's law: 1. Laboratory measurements. Journal of Geophysical Research: Solid Earth, 99, 11897-11911

Salvaggio, C., \& Miller, C.J. (2001). Methodologies and protocols for the collection of midwave and longwave infrared emissivity spectra using a portable field spectrometer. In, Aerospace/Defense Sensing, Simulation, and Controls (pp. 539-548): International Society for Optics and Photonics

Savitzky, A., \& Golay, M.J. (1964). Smoothing and differentiation of data by simplified least squares procedures. Analytical chemistry, 36, 1627-1639 
Schlerf, M., Atzberger, C., \& Hill, J. (2005). Remote sensing of forest biophysical variables using HyMap imaging spectrometer data. Remote Sensing of Environment, 95, 177-194

Schmidt, G.A., Shindell, D.T., Miller, R.L., Mann, M.E., \& Rind, D. (2004). General circulation modelling of Holocene climate variability. Quaternary Science Reviews, 23, 2167-2181

Schmidt, K., \& Skidmore, A. (2003). Spectral discrimination of vegetation types in a coastal wetland. Remote Sensing of Environment, 85, 92-108

Seemann, S.W., Borbas, E.E., Knuteson, R.O., Stephenson, G.R., \& Huang, H.L. (2008). Development of a global infrared land surface emissivity database for application to clear sky sounding retrievals from multispectral satellite radiance measurements. Journal of Applied Meteorology and Climatology, $47,108-123$

Sellers, P. (1987). Canopy reflectance, photosynthesis, and transpiration, II. The role of biophysics in the linearity of their interdependence. Remote Sensing of Environment, 21, 143-183

Sellers, P.J. (1985). Canopy reflectance, photosynthesis and transpiration. International Journal of Remote Sensing, 6, 1335-1372

Sepulcre-Cantó, G., Zarco-Tejada, P.J., Jiménez-Muñoz, J., Sobrino, J., De Miguel, E., \& Villalobos, F. (2006). Detection of water stress in an olive orchard with thermal remote sensing imagery. Agricultural and Forest Meteorology, 136, 31-44

Serrano, L., Ustin, S.L., Roberts, D.A., Gamon, J.A., \& Penuelas, J. (2000). Deriving water content of chaparral vegetation from AVIRIS data. Remote Sensing of Environment, 74, 570-581

Shao, J. (1993). Linear model selection by cross-validation. Journal of the American statistical Association, 88, 486-494

Skidmore, A., Turner, B., Brinkhof, W., \& Knowles, E. (1997). Performance of a neural network: mapping forests using GIS and remotely sensed data. Photogrammetric engineering and remote sensing, 63, 501-514

Skidmore, A.K., Pettorelli, N., Coops, N.C., Geller, G.N., \& Matthew, H. (2015). Agree on biodiversity metrics to track from space: ecologists and space agencies must forge a global monitoring strategy. Nature, 523, 403-406

Sobhan, I. (2007). Species discrimination from a hyperspectral perspective

Sobrino, J. (1989). Desarrollo de un modelo teórico para implementar la medida de la temperatura realizada mediante teledetección. Aplicación a un campo de naranjos. In: PhD dissertation, University of Valencia, Valencia, Spain 
Sobrino, J., Caselles, V., \& Becker, F. (1990). Significance of the remotely sensed thermal infrared measurements obtained over a citrus orchard. ISPRS Journal of Photogrammetry and Remote Sensing, 44, 343-354

Sobrino, J., \& Raissouni, N. (2000). Toward remote sensing methods for land cover dynamic monitoring: application to Morocco. International Journal of Remote Sensing, 21, 353-366

Sobrino, J., Raissouni, N., \& Li, Z.-L. (2001). A comparative study of land surface emissivity retrieval from NOAA data. Remote Sensing of Environment, 75, 256-266

Sobrino, J.A., Jiménez-Muñoz, J.C., \& Paolini, L. (2004). Land surface temperature retrieval from LANDSAT TM 5. Remote Sensing of Environment, 90, 434-440

Sobrino, J.A., Jiménez-Muñoz, J.C., Sòria, G., Romaguera, M., Guanter, L., Moreno, J., Plaza, A., \& Martínez, P. (2008). Land surface emissivity retrieval from different VNIR and TIR sensors. IEEE Transactions on Geoscience and Remote Sensing, 46, 316-327

Sobrino, J.A., Jiménez-Muñoz, J.C., \& Verhoef, W. (2005). Canopy directional emissivity: Comparison between models. Remote Sensing of Environment, 99, 304-314

Sobrino, J.A., Jiménez-Muñoz, J.C., Labed-Nachbrand, J., \& Nerry, F. (2002). Surface emissivity retrieval from digital airborne imaging spectrometer data. Journal of Geophysical Research: Atmospheres, 107

Stoms, D.M., \& Estes, J. (1993). A remote sensing research agenda for mapping and monitoring biodiversity. International Journal of Remote Sensing, 14, 1839-1860

Suárez, L., Zarco-Tejada, P.J., Sepulcre-Cantó, G., Pérez-Priego, O., Miller, J., Jiménez-Muñoz, J., \& Sobrino, J. (2008). Assessing canopy PRI for water stress detection with diurnal airborne imagery. Remote Sensing of Environment, 112, 560-575

Sun, Y., Liu, X., Wu, Y., \& Liao, C. (2008). Identifying hyperspectral characters of wetland species using in situ data. Int Arch Photogramm Remote Sens Spa Inf Sci, 107, 459-465

Szakiel, A., Pączkowski, C., Pensec, F., \& Bertsch, C. (2012). Fruit cuticular waxes as a source of biologically active triterpenoids. Phytochemistry Reviews, 11, 263-284

Thenkabail, P.S., Smith, R.B., \& De Pauw, E. (2000). Hyperspectral vegetation indices and their relationships with agricultural crop characteristics. Remote Sensing of Environment, 71, 158-182 
Trombetti, M., Riaño, D., Rubio, M., Cheng, Y., \& Ustin, S. (2008). Multitemporal vegetation canopy water content retrieval and interpretation using artificial neural networks for the continental USA. Remote Sensing of Environment, 112, 203-215

Tsai, F., Lin, E.K., \& Yoshino, K. (2007). Spectrally segmented principal component analysis of hyperspectral imagery for mapping invasive plant species. International Journal of Remote Sensing, 28, 1023-1039

Tucker, C.J. (1979). Red and photographic infrared linear combinations for monitoring vegetation. Remote Sensing of Environment, 8, 127-150

Tucker, C.J. (1980). Remote sensing of leaf water content in the near infrared. Remote Sensing of Environment, 10, 23-32

Turner, D.P., Cohen, W.B., Kennedy, R.E., Fassnacht, K.S., \& Briggs, J.M. (1999). Relationships between leaf area index and Landsat TM spectral vegetation indices across three temperate zone sites. Remote Sensing of Environment, 70, 52-68

Ullah (2013). Thermal plants: characterizing vegetation parameters using mid to thermal infrared hyperspectral remote sensing. In: Universiteit Twente

Ullah, S., Schlerf, M., Skidmore, A.K., \& Hecker, C. (2012a). Identifying plant species using mid-wave infrared $(2.5-6 \mu \mathrm{m})$ and thermal infrared $(8-14 \mu \mathrm{m})$ emissivity spectra. Remote Sensing of Environment, 118, 95-102

Ullah, S., Skidmore, A.K., Groen, T.A., \& Schlerf, M. (2013). Evaluation of three proposed indices for the retrieval of leaf water content from the mid-wave infrared $(2-6 \mu \mathrm{m})$ spectra. Agricultural and Forest Meteorology, 171, 65-71

Ullah, S., Skidmore, A.K., Naeem, M., \& Schlerf, M. (2012b). An accurate retrieval of leaf water content from mid to thermal infrared spectra using continuous wavelet analysis. Science of the total environment, 437, 145-152

Ullah, S., Skidmore, A.K., Ramoelo, A., Groen, T.A., Naeem, M., \& Ali, A. (2014). Retrieval of leaf water content spanning the visible to thermal infrared spectra. ISPRS Journal of Photogrammetry and Remote Sensing, 93, 56-64

Ustin, S.L., Roberts, D.A., Pinzon, J., Jacquemoud, S., Gardner, M., Scheer, G., Castaneda, C.M., \& Palacios-Orueta, A. (1998). Estimating canopy water content of chaparral shrubs using optical methods. Remote Sensing of Environment, 65, 280-291

Valor, E., \& Caselles, V. (1996). Mapping land surface emissivity from NDVI: Application to European, African, and South American areas. Remote Sensing of Environment, 57, 167-184 
Van de Griend, A., \& Owe, M. (1993). On the relationship between thermal emissivity and the normalized difference vegetation index for natural surfaces. International Journal of Remote Sensing, 14, 1119-1131

van den Hurk, B.J., Viterbo, P., \& Los, S.O. (2003). Impact of leaf area index seasonality on the annual land surface evaporation in a global circulation model. Journal of Geophysical Research: Atmospheres (1984-2012), 108

Van der Meer, F.D., Van der Werff, H.M., van Ruitenbeek, F.J., Hecker, C.A., Bakker, W.H., Noomen, M.F., van der Meijde, M., Carranza, E.J.M., de Smeth, J.B., \& Woldai, T. (2012). Multi-and hyperspectral geologic remote sensing: A review. International journal of applied Earth observation and geoinformation, 14, 112-128

Verbesselt, J., Somers, B., Lhermitte, S., Jonckheere, I., Van Aardt, J., \& Coppin, P. (2007). Monitoring herbaceous fuel moisture content with SPOT VEGETATION time-series for fire risk prediction in savanna ecosystems. Remote Sensing of Environment, 108, 357-368

Vertessy, R., Benyon, R., O'sullivan, S., \& Gribben, P. (1995). Relationships between stem diameter, sapwood area, leaf area and transpiration in a young mountain ash forest. Tree Physiology, 15, 559-567

Vierling, L.A., Fersdahl, M., Chen, X., Li, Z., \& Zimmerman, P. (2006). The Short Wave Aerostat-Mounted Imager (SWAMI): A novel platform for acquiring remotely sensed data from a tethered balloon. Remote Sensing of Environment, 103, 255-264

Viña, A., Gitelson, A.A., Nguy-Robertson, A.L., \& Peng, Y. (2011). Comparison of different vegetation indices for the remote assessment of green leaf area index of crops. Remote Sensing of Environment, 115, 3468-3478

Wan, Z., \& Dozier, J. (1996). A generalized split-window algorithm for retrieving land-surface temperature from space. IEEE Transactions on Geoscience and Remote Sensing, 34, 892-905

Wan, Z., Wang, P., \& Li, X. (2004). Using MODIS land surface temperature and normalized difference vegetation index products for monitoring drought in the southern Great Plains, USA. International Journal of Remote Sensing, $25,61-72$

Wang, Y., Woodcock, C.E., Buermann, W., Stenberg, P., Voipio, P., Smolander, H., Häme, T., Tian, Y., Hu, J., \& Knyazikhin, Y. (2004). Evaluation of the MODIS LAI algorithm at a coniferous forest site in Finland. Remote Sensing of Environment, 91, 114-127

Wang, Z., Skidmore, A.K., Wang, T., Darvishzadeh, R., \& Hearne, J. (2015). Applicability of the PROSPECT model for estimating protein and 
cellulose+lignin in fresh leaves. Remote Sensing of Environment, 168, 205218

Watson, D.J. (1947). Comparative physiological studies on the growth of field crops: I. Variation in net assimilation rate and leaf area between species and varieties, and within and between years. Annals of botany, 41-76

Whitmore, T., Brown, N., Swaine, M., Kennedy, D., Goodwin-Bailey, C., \& Gong, W.-K. (1993). Use of hemispherical photographs in forest ecology: measurement of gap size and radiation totals in a Bornean tropical rain forest. Journal of tropical ecology, 9, 131-151

Williams, P., \& Norris, K. (1987). Near-infrared technology in the agricultural and food industries. American Association of Cereal Chemists, Inc.

Wong, C., \& Blevin, W. (1967). Infrared reflectances of plant leaves. Australian Journal of Biological Sciences, 20, 501-508

Yebra, M., Dennison, P.E., Chuvieco, E., Riaño, D., Zylstra, P., Hunt, E.R., Danson, F.M., Qi, Y., \& Jurdao, S. (2013). A global review of remote sensing of live fuel moisture content for fire danger assessment: Moving towards operational products. Remote Sensing of Environment, 136, 455-468

Yoder, B.J., \& Waring, R.H. (1994). The normalized difference vegetation index of small Douglas-fir canopies with varying chlorophyll concentrations. Remote Sensing of Environment, 49, 81-91

Zarco-Tejada, P.J., Miller, J., Mohammed, G., Noland, T., \& Sampson, P. (2002). Vegetation stress detection through chlorophyll+ estimation and fluorescence effects on hyperspectral imagery. Journal of environmental quality, 31, 1433-1441

Zarco-Tejada, P.J., Miller, J.R., Noland, T.L., Mohammed, G.H., \& Sampson, P.H. (2001). Scaling-up and model inversion methods with narrowband optical indices for chlorophyll content estimation in closed forest canopies with hyperspectral data. IEEE Transactions on Geoscience and Remote Sensing, 39, 1491-1507

Zarco-Tejada, P.J., Rueda, C., \& Ustin, S. (2003). Water content estimation in vegetation with MODIS reflectance data and model inversion methods. Remote Sensing of Environment, 85, 109-124

Zheng, G., \& Moskal, L.M. (2009). Retrieving leaf area index (LAI) using remote sensing: theories, methods and sensors. Sensors, 9, 2719-2745

Zhou, Q., Robson, M., \& Pilesjo, P. (1998). On the ground estimation of vegetation cover in Australian rangelands. International Journal of Remote Sensing, 19, 1815-1820 


\section{Summary}

There is an increasing demand for understanding and evaluating the impact of climate change and global warming on the Earth system. Remote sensing as a non-destructive and cost-effective approach is one of the most valuable tools to understanding the ecosystems changes.

This dissertation aims to contribute to understanding the potential of thermal (TIR, 8-14 $\mu \mathrm{m}$ ), hyperspectral, and multispectral remote sensing data to quantify two vegetation variables at canopy level: leaf area index (LAI) and vegetation water content in terms of fuel moisture content $\left(\mathrm{FMC}_{\mathrm{C}}\right)$ and equivalent water thickness $\left(\mathrm{EWT}_{\mathrm{C}}\right)$. The research investigated the relation between LAI and emissivity spectra, as well as the applicability of emissivity spectra to retrieve the LAI, FMC , and EWTC benefiting from empirical models under laboratory controlled conditions over TIR region.

The laboratory experiments demonstrated that there exists a positive correlation between emissivity spectra and LAI. It was concluded that LAI could be successfully predicted at a moderate accuracy using empirical models. The findings revealed that multivariate techniques are more reliable to retrieve LAI in comparison with univariate statistical approaches using TIR data. The results of the study showed that $\mathrm{FMC}_{\mathrm{C}}$ and $\mathrm{EWT}_{\mathrm{C}}$ could be successfully estimated through artificial neural networks, and $\mathrm{FMC}_{\mathrm{C}}$ was more accurately predicted than EWTC. The findings of the research confirmed that TIR remotely sensed data are species-specific regarding prediction of vegetation biophysical as well as biochemical variables at canopy level.

Furthermore, research has moved on the spaceborne level to predict LAI coupled with univariate and machine learning approaches, which have benefitted from TIR multispectral data. The results revealed that LAI could be predicted using land surface emissivity (LSE) with reasonable accuracy. The LSE was found to be correlated with the plot vegetation type. The findings of this thesis confirmed that different vegetation indices achieved similar results for retrieval of LAI using TIR hyperspectral and multispectral data. Lastly, the results showed that the combination of reflectance and emissivity data could improve the prediction accuracy of LAI. This thesis extends the existing knowledge of applied research in vegetation remote 
sensing studies from the TIR perspective. The findings and approaches of this thesis have confirmed the potential of TIR hyperspectral remote sensing data to generate valuable information regarding vegetation characteristics at canopy level under laboratory conditions. The results further revealed the potential of TIR remote sensing data to retrieve LAI as a primary vegetation biophysical variable over the mixed temperate forest at satellite level. These achievements will improve our knowledge, and enhance our understanding of biodiversity through monitoring and assessment of changes in essential biodiversity variables such as LAI. 


\section{Samenvatting}

Er is steeds meer vraag naar het begrijpen en het evalueren van de impact van klimaatverandering en de opwarming van de aarde. Remote sensing als een niet-destructieve en kosteneffectieve aanpak is hierbij een van de meest waardevolle tools om de veranderingen in de ecosystemen te begrijpen.

Dit proefschrift streeft ernaar bij om het potentieel van thermische infrarood (TIR, 8-14 $\mu \mathrm{m}$ ), hyperspectrale en multispectrale afstandswaarnemingsgegevens te begrijpen om twee vegetatievariabelen op luifelniveau te kwantificeren, namelijk de blad-oppervlakte index (LAI) en het vegetatie watergehalte, gemeten in termen van Brandstof vochtgehalte $\left(\mathrm{FMC}_{\mathrm{C}}\right)$ en gelijkwaardige waterdikte $\left(\mathrm{EWT}_{\mathrm{C}}\right)$. Het onderzoek onderzocht de relatie tussen LAI en emissiviteitsspectra, evenals de toepasbaarheid van emissiviteitsspectra om de $\mathrm{LAI}, \mathrm{FMC}_{\mathrm{C}}$ en $\mathrm{EWT}_{\mathrm{C}}$ te verkrijgen vanuit de onder laboratorium gecontroleerde omstandigheden- empirische modellen in de TIR-regio.

De laboratorium experimenten hebben aangetoond dat er een positieve correlatie bestaat tussen emissiviteitsspectra en LAI. Er werd geconcludeerd dat LAI met succes empirische modellen zou kunnen voorspellen met een gematigde nauwkeurigheid. Uit de bevindingen bleek dat multivariate technieken betrouwbaarder zijn om LAI te verkrijgen in vergelijking met univariate statistische benaderingen die gebruik maken van TIR data. De resultaten van de studie toonden aan dat $\mathrm{FMC}_{\mathrm{C}}$ en EWT $_{\mathrm{C}}$ succesvol kunnen worden geschat door middel van kunstmatige neurale netwerken, en FMCc werd nauwkeuriger voorspeld dan EWTC. De bevindingen van het onderzoek hebben bevestigd dat TIR-afstandsgevoede gegevens specifiek zijn voor het voorspellen van vegetatie biofysische en biochemische variabelen op luifelniveau.

Daarna is het onderzoek verplaatst naar het ruimtelijk niveau om LAI te voorspellen in combinatie met univariate en "machine-learning" benaderingen, vanuit de TIR multispectrale data. Uit de resultaten blijkt dat LAI met redelijke nauwkeurigheid kan worden voorspeld met behulp van landingsemissiviteit (LSE). De LSE bleek gecorreleerd te zijn aan het vegetatie type in een bepaald gebied. De bevindingen van dit proefschrift bevestigden dat verschillende vegetatie-indices vergelijkbare resultaten 
hebben verkregen voor het verkrijgen van LAI met behulp van TIR hyperspectrale en multispectrale data. Ten slotte bleek dat de combinatie van reflectie- en emissiviteitsgegevens de voorspellingsnauwkeurigheid van LAI zou kunnen verbeteren.

Dit proefschrift breidt de bestaande kennis uit van toegepast onderzoek in vegetatie-remote sensing studies uit het TIR perspectief. De bevindingen en benaderingen van dit proefschrift hebben het potentieel van TIR hyperspectrale afstandswaarnemingsgegevens bevestigd om waardevolle informatie te genereren over vegetatiekenmerken op luifelniveau onder laboratoriumomstandigheden. De resultaten onthullen daarnaast het potentieel van TIR remote sensing data om LAI te verkrijgen op satellietniveau als een biofysische variabele van de vegetatie in het gemengde gematigde bos. Deze prestaties zullen onze kennis verbeteren en ons begrip van biodiversiteit verbeteren door het monitoren en evalueren van veranderingen in essentiële biodiversiteitsvariabelen, zoals LAI. 


\section{Biography}

Elnaz Neinavaz was born on $12^{\text {th }}$ May 1982 in Tehran, Iran. She received a B.Sc in a Natural Resources Engineering- Environment at the Azad University- North Tehran Branch and obtained her M.Sc. in Natural Resources Engineering- Habitats and Biodiversity from the Azad UniversityScience and Research Tehran Branch. After graduation, she started to collaborate with the United Nations Development Program (UNDP), Global Environment Facility (GEF)/ Small Grant Program (SGP) and Plan for the Land Society on the number of the national and international environmental projects for several years. In 2013, she was awarded the European Commission, Erasmus Mundus Scholarship to peruse her doctoral research at the Faculty of Geo- Information Science and Earth Observation (ITC), University of Twente, which resulted in this thesis

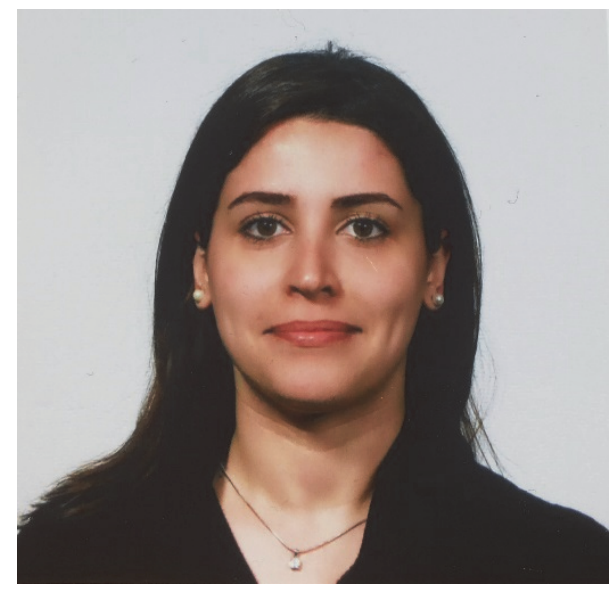
and the following publications:

\section{Scientific publications:}

Neinavaz, E., Darvishzadeh, R., Skidmore, A.K., \& Groen, T.A. (2016). Measuring the Response of Canopy Emissivity Spectra to Leaf Area Index Variation using Thermal Hyperspectral Data. International Journal of Applied Earth Observation and Geoinformation 50. 40-47.

Neinavaz, E., Skidmore, A.K., Darvishzadeh, R., \& Groen, T.A. (2016). Leaf Area Index Retrieved from Thermal Hyperspectral Data. ISPRS-International Archives of Photogrammetry, remote Sensing and Spatial Information Science, 99-105.

Neinavaz, E., Skidmore, A.K., Darvishzadeh, R., \& Groen, T.A. (2016). Retrieval of Leaf Area Index in Different Plant Species using Thermal Hyperspectral Data. ISPRS Journal of Photogrammetry and remote Sensing, 119, 390-401. 
Neinavaz, E., Skidmore, A.K., Darvishzadeh, R., \& Groen, T.A. Retrieving Vegetation Canopy water Content from Hyperspectral Thermal Measurements. Agriculture and Forest Meteorology (In review, after revision).

Neinavaz, E., Darvishzadeh, R., Skidmore, A.K., \& Abdullah, H. Successful Retrieval of Leaf Area Index from Thermal Satellite Imagery over Mixed Temperate Forest. Remote Sensing of Environment. (In review).

\section{International conferences:}

Neinavaz, E., Darvishzadeh, R., Skidmore, A.K., Groen, and T.A., Hecker, C. Estimation of Leaf Area Index from Hyperspectral Thermal Data. $9^{\text {th }}$ EARSeL SIG Imaging Spectroscopy Workshop, 14-16 April 2015, Luxemburg.

Neinavaz, E., Skidmore, A.K., Darvishzadeh, R., \& Groen, T.A. Retrieval of Leaf Area Index in Different Plant Species using Thermal Hyperspectral Data. XXIII ISPRS Congress, 12-16 July 2016, Prague, Czech Republic.

Neinavaz, E., Skidmore, A.K., Darvishzadeh, R., \& Groen, T.A. Vegetation Water Content Retrieved using Thermal Hyperspectral Data. $10^{\text {th }}$ EARSel SIG Imaging Spectroscopy Workshop, 19-21 April 2017, Zurich, Switzerland. 


\section{ITC Dissertation List}

https://www.itc.nl/Pub/research_programme/Research-review-andoutput/PhD-Graduates 